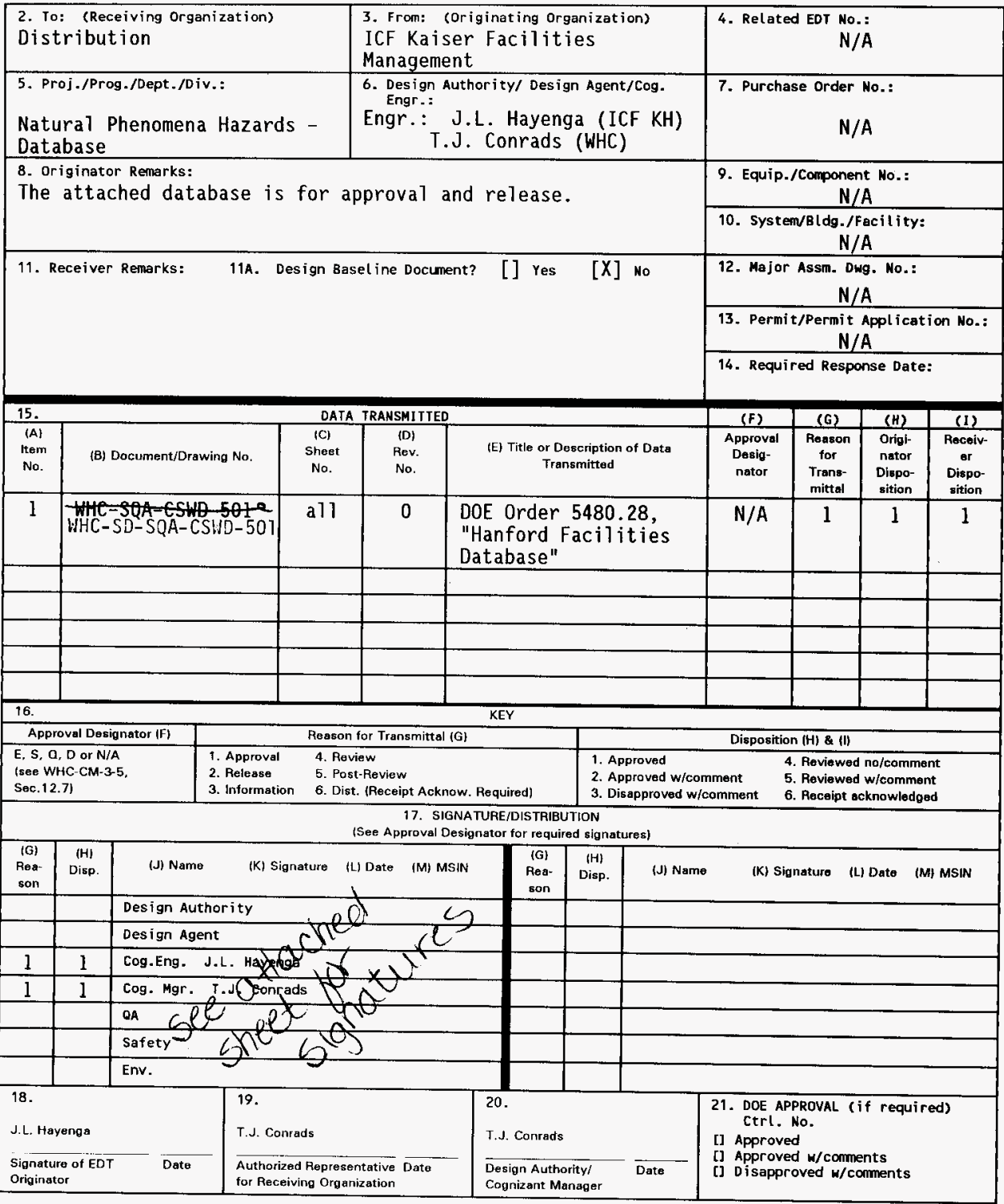


EDT 618828

Page 2 of 2

\begin{tabular}{|l|l|}
\hline $\begin{array}{l}\text { 2. Io: (Receiving organization) } \\
\text { Distribution }\end{array}$ & $\begin{array}{l}\text { 3. From: Coriginating Organization) } \\
\text { ICF Kaiser Facilities } \\
\text { Management }\end{array}$ \\
\hline 5. Proj./Prog./Dept./Div.: & $\begin{array}{l}\text { 6. Design Authority/ Design Agent/Cog. } \\
\text { Engr.: J.L. Hayenga (ICF KH) } \\
\text { Natural Phenomena Hazards - }\end{array}$ \\
$\begin{array}{l}\text { Engr.: J.J. Conrads (WHC) } \\
\text { Database }\end{array}$
\end{tabular}

8. Originator Remarks:

The attached database is for approval and release.

11. Receiver Remarks: 11A. Design Baseline Document? [] Yes [X] No

4. Related EDT No.:

$N / A$

7. Purchase Order No.:

N/A

9. Equip./Component No.:

N/A

10. System/Btdg./Facility:

N/A

12. Major Assm. Dwg. No.:

$N / A$

13. Permit/Permit Applicetion No.: N/A

14. Required Response Date:

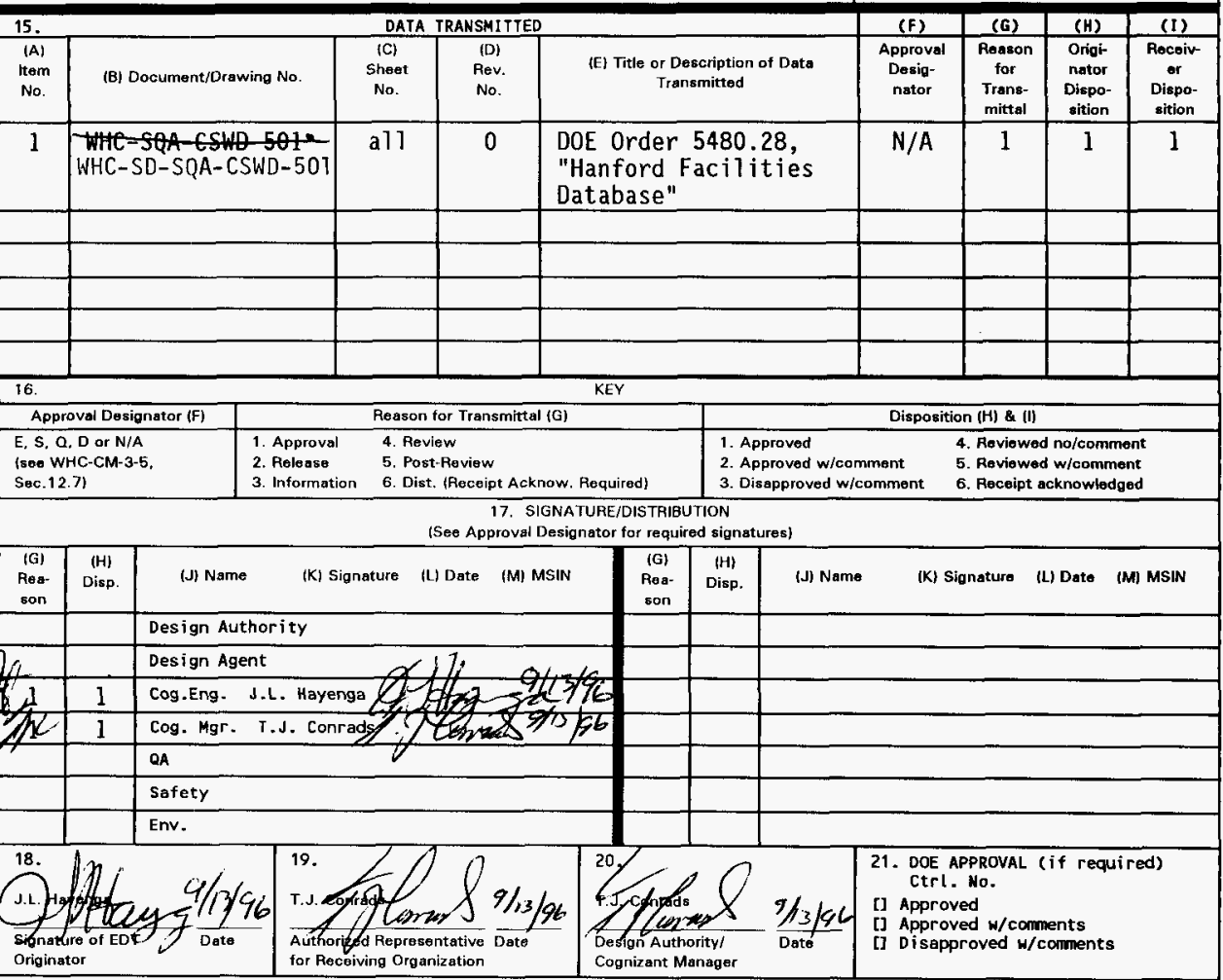




\title{
DOE Order 5480.28 Hanford Facilities Database
}

\author{
J. L. Hayenga
}

West inghouse Hanford Company, Richland, WA 99352

U.S. Department of Energy Contract DE-AC06-87RL10930

EDT/ECN: $\quad 618828$

Org Code: 52910

UC: 620

B\&R Code: YNO100000

Charge Code: E30732

Tota7 Pages: 115

Key Words: Natural Phenomena Hazard, NPH, Existing Hanford Facilities, Database, Prioritization, DOE Order 5480.28

Abstract: This document describes the development of a database of DOE and/or leased Hanford Site Facilities. The completed database will consist of structure/facility parameters essential to the prioritization of these structures for natural phenomena hazard vulnerability in compliance with DOE Order 5480.28, Natural Phenomena Hazards Mitigation. The prioritization process will be based upon the structure/facility vulnerability to natural phenomena hazards. The ACCESS based database, "Hanford Facilities Site Database", is generated from current Hanford site information and databases.

TRADEMARK DISCLAIMER. Reference herein to any specific commercial product, process, or service by trade name, trademark, manufacturer, or otherwise, does not necessarily constitute or imply its endorsement, recomendation, or favoring by the United States Government or any agency thereof or its contractors or subcontractors.

Printed in the United States of America. To obtain copies of this document, contact: WHC/BCS

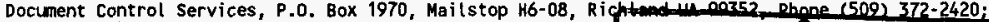
Fax (509) 376-4989.
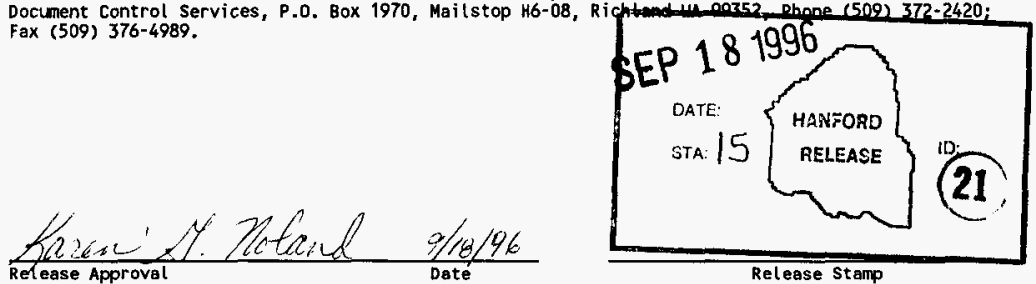

Release Stamp 
WHC-SD-SQA-CSWD-501 Rev 0

HANFORD FACILITIES DATABASE

J. L. Hayenga and C. H. Dohrer

September 1996 


\section{EXECUTIVE SUMMARY}

Department of Energy (DOE) Order 5480.28, Natural Phenomena Hazard Mitigation. transmitted to Westinghouse Hanford Company (WHC) for compliance includes stringent new natural phenomena hazard (NPH) criteria for evaluating the vulnerability of existing DOE facilities to NPH. Within the order is the requirement to develop a prioritized database of existing facilities/ structures (DOE owned or leased) for future NPH evaluation and potential upgrade. The database is to include a listing of all structures/buildings within the above category and will identify parameters necessary for the review and prioritization of the structures/buildings based upon NPH vulnerability. This document describes the development of the format and includes some completed fields of the database.

Original planning identified the "Facilities Information Management System (FIMS)" database as a potential source for establishment of the database. FIMS is not operational for the Hanford Site at this time. The "Richland Property System (RLPS)" and "FacilitiesCORE" databases are the primary sources used to generate the building and structure database.

The generated database, "Hanford Facilities Site Database (HFSD)" is the result of the integration of the RLPS and Facilities Core database essential data into one site wide repository to enable the initiation of the evaluation of the structure/building vulnerability to NPH. The prioritization values will also be entered into the HFSD. As FIMS becomes accessible to the Hanford Site, the data from HFSD could also be used to update key FIMS data fields and provide the basis for the final Hanford Site report of the NPH evaluations of the Hanford Site structures. 
WHC-SD-SQA-CSWD-501 Rev 0

Table of Contents

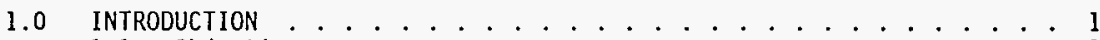

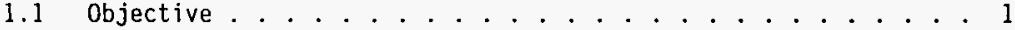

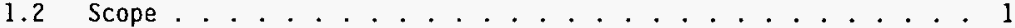

1.3 Inventory Completeness .............. 1

1.4 Types of Structures Included in Inventory .........2

2.0 Inventory database ................ 3

2.1 Relationship to Existing Hanford Databases ......... . 3

2.2 Data Entry, Reports . . . . . . . . . . . . 4

Appendix A

Data Entry Screen

Appendix B

Definitions and Source Data

Appendix C

Data Structure

Appendix D

Facility Listing

Appendix $E$

Facility Demolition List 
WHC.SD-SQA-CSWD-501 ReV 0

HANFORD FACILITIES DATABASE

\subsection{INTRODUCTION}

The U. S. Department of Energy Order 5480.28, "Natural Phenomena Hazards Mitigation," establishes requirements to assess existing Hanford structures and determine their NPH vulnerability. An initial step in the assessment is to inventory the Hanford Site buildings and structures together with specific structural and mission data. With this data, the buildings and structures will be prioritized based upon their susceptibility to NPH effects. This document identifies the source, scope, and quality of data utilized to develop the database. The developed database will also be used to document the results of the NPH assessments and prioritization.

\subsection{OBJECTIVE}

The objective of this report is to document the process utilized to identify and inventory Hanford structures. A secondary objective is the development of information to be used in the NPH assessment and prioritization processes. The information within this database may be integrated into the FIMS database for final reporting, if required.

\subsection{SCOPE}

The inventory of structures is 1 imited to those for which DOE has assigned management responsibility to WHC, ICF/KH and BCSR. The facilities include government owned, commercially leased and General Services Administration (GSA) leased facilities. The Federal Building, although a GSA leased facility is not included in this inventory. All other facilities on the Hanford Site will be the responsibility of other DOE contractors.

DOE Order 5480.28 is the source for the database requirement. The current format is designed only to document the results of the Prioritization Phase of order implementation. Additional data will be required to support the followon phases including the Evaluation and Upgrade Phases. Appendix A defines the current data entry screens.

\subsection{INVENTORY COMPLETENESS}

The HFSD is developed from two on-site databases, RLPS ${ }^{1}$ and FacilitiesCORE ${ }^{2}$. RLPS is the repository for property data at the Hanford Site. Responsibility as well as accounting, location, historical and other descriptive information is recorded for each asset on the site. Attachment B, "Definitions and Sources Data," displays the fields available in the database. The RLPS was used to initiate the inventory listing. FacilitiescoRE and other on-site databases were used to provide supplementary information and cross-checks on the RLPS data. The operating procedures for RLPS and FacilitiesCORE are provided in References 1 and 2, respectively. 
The HFSD inventory listing included as Appendix D was generated in-total from the referenced databases. To date there have been no specific efforts to inventory the list of structures other than the normal inventory and control efforts. The RLPS database is verified on a predetermined basis. This date is included in the database as the "Last Inventoried" date. Field surveys will be required to collect additional data and complete the database.

In some cases the "Facility Number" relates to only a part of the facility. For example, a covered loading-dock built after the main structure may have a separate facility identification number. These structures were not identified separately from the main facility. A comparison of original source data and HFSD may not match due to these type of cited inconsistencies. Additionally, the inventory of Hanford Site facilities is in a state of continual change. There is a continuing process of transferring facilities to other governmental agencies or to another contractors. Also there is a continuing process of demolition. Appendix $F$ is the current list of facilities to be demolished.

\subsection{TYPES OF STRUCTURES INCLUDED IN INVENTORY}

The database includes only buildings and structures which are described as any structure with four walls, roof and floor that is intended to house people, store material or shelter a process. Therefore tanks, wells, bridges, electrical substations, roads or rail tracks (railroads) are not included.

Also buildings which are contained within the fence lines of a tank farm compound are excluded from the database. These structures are usually less than 10 square meters under roof and are typically used as compressor sheds, pump houses, etc. 


\subsection{INVENTORY DATABASE}

Microsoft Access ${ }^{\text {rM }}$ is the database management software used to develop the Hanford Facilities Site Database. The HFSD is primarily a repository for assembling the data for the Assessment and Prioritization Phases of DOE Order 5480.28 implementation. This initial database system was not constructed to current internal standards for software control. HFSD currently resides on an HLAN server. It is protected by account number and password security at the server leve]. Typical database services like backup, security, etc. are provided for HFSD as a normal HLAN function. Additional administrative controls will be applied to the database depending on future requirements and implementation.

\subsection{RELATIONSHIP TO EXISTING HANFORD DATABASES}

The source data for HFSD was assembled from several databases, see Appendix $B$. RLPS tracks all property assigned to WHC and its subcontractors ICF/KH and BCSR. Within this database are a vast number of records that do not equate to either a facility/structure or a physical quantity. The record coding has left some ambiguity as to the nature of an item being tracked. A significant portion of the activity in assembling HFSD was the "weeding out" of records that did not equate to a facility/structure. This initial database, HFSD, does represent the best available list of Hanford Site facilities. The initial source of data load scripts were retained with the database to allow the source data to be reloaded at some future date, if required. All database entries, during the future phases of order implementation, will be verified.

\subsection{DATA ENTRY, REPORTS}

Appendix A presents a data entry screen which has been developed to allow the system to be updated with additional data required for NPH assessment and prioritization. An initial draft report has been generated (Appendix D) to ist the current inventory of structures and available data.

\subsection{REFERENCES}

1.0 WHC-CM-2-3, Property Management Manual

2.0 WHC-CM-8-7, Section 907 Building Number Assignment and Requirements

3.0 DOE Order 5480.28, "Natural Phenomena Hazards Mitigation," (1-15-96), U.S. Department of Energy, Washington, D.C.

$T M$ Microsoft Access is a registered trademark of the Microsoft Corporation. 
WHC-SD-SQA-CSWD-501 Rev 0

Appendix A

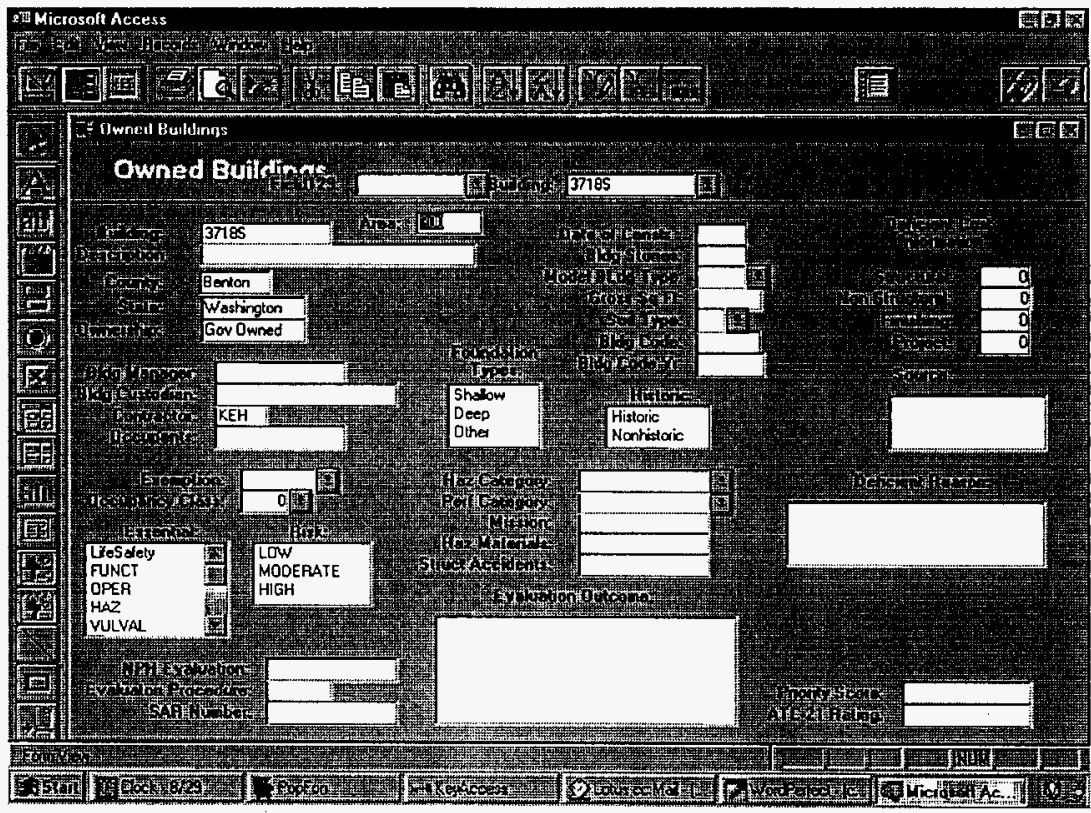

Data Entry Screen

Appendix A-1 


\section{WHC-SD-SQA-CSWD-501 Rev 0 \\ Appendix B \\ DEFINITIONS \& SOURCES DATA}

\begin{tabular}{|c|c|c|c|c|}
\hline Field Name & Definition & Source & Date & Hssfd Field \\
\hline AREA & Hanford site Area & Rlps Bldg Table & $8 / 21$ & Area \\
\hline Bldg & Facility Number & Rlps Bldg Table & $8 / 21$ & Building \\
\hline Prname & Property Name & Rlps Bldg Table & $8 / 21$ & \\
\hline Prtype & Property Type & Rlps Bldg Table & $8 / 21$ & Description \\
\hline Fcname & Facility Core Name & $\mathrm{Hfcr} 204 \mathrm{r}$ & $8 / 23$ & Description \\
\hline Prnum & Property Record Number & Rlps Record & $8 / 21$ & \\
\hline $\operatorname{cont}$ & Contractor Division & Rlps Bldg Table & $8 / 21$ & Contractor \\
\hline Pringr & Property Level $2 \mathrm{Mgr}$ & Rlps Bldg Table & $8 / 21$ & \\
\hline F cmngr & Facility Core Manager & Hfer203a & $8 / 23$ & Bldg Manager \\
\hline fesize & Facility Core Square Feet & $H f e r 204 r$ & $8 / 23$ & Gross Sq Ft \\
\hline Prsize & Property Square footage & Rlps Record & $8 / 21$ & \\
\hline Built & Property in Service Date & Rlps Record & $8 / 21$ & Date of Construction \\
\hline Custorg & Assigned Custodial Code & Rlps Record & $8 / 21$ & \\
\hline Asset & Property Asset Type & Rlps Record & $8 / 21$ & \\
\hline Level2 & Level 2 Org Code Manager & Hpcr442r & $8 / 26$ & Bldg Custodian \\
\hline Orgname & Level 2 Organization Name & Hpcr $442 r$ & $8 / 26$ & \\
\hline occ? & Occupied (Yes/blank) & HFCR208R & $8 / 26$ & \\
\hline Location Cnt & Count of Names within a Bldg & HFCR208R & $7 / 20$ & Occupants \\
\hline
\end{tabular}


WHC-SD-SQA-CSWD-501 Rev 0

EXPANDED DEFINITION AND METHODOLOGY

\section{RLPS Building Table}

The RLPS "Building Table" is a check table that is used to validate property locations. It contains all possible facility locations where individual DOE owned assets may be located both on and off the Hanford Site.

The initial download of 2,407 entries was reduced to 947 entries by the following process of elimination to remove entries that we do not want on the completed file.

B lank lines and off-site locations were eliminated by deleting for Area<100B, Area $>$ RCHN, Area $>700 \&<K E N N$, Area $=M S C, 0 S, P T L, \& Q S O$. Result $=1941$ records .

Other contractor assets were eliminated by deleting for cont $=E R C$, Cont $=0$, Custorg $=D$, Custorg $=B$. Result $=1531$ records.

Generic property locations were eliminated by deleting for Bldg=N, P, S, R, W, $>8,\langle M$, and buildings within Tank Farms were removed by deleting for $B 1 d g=241$. Result $=1384$ records .

Balance of assets eliminated were deleted by examination of names, IE tanks, cribs, burial grounds, pads, vaults, pits, etc and deleting the entries accordingly. Result $=947$ records.

Those records not displaying a Property number (Fxxxxxx, FAxxxxx, or M0xxxxx) were researched and property record number was added to facilitate entering information from other property record fields. There are 35 records where a property record number was not located but the asset appeared to be existing buildings or facilities that should be researched further.

Two fields from the RLPS Building Table require caveats. The CONT and PRMNGR fields are currently being reconciled. These fields correspond to the LEVEL2 and ORGNAME fields that are derived from the organization code assigned by property record. While the majority of these fields do agree it should be noted that when they do not agree the custodial organization code is the authority for asset responsibility. A third field "ASSET" is included for reference and cross-check only. Note that the code for buildings is 501 , but a few without this code were included because other information indicated that it could be a building.

\section{HFCR204R}

HFCR204R is a Soft Reporting table that contains all Hanford Site Facility numbers that are used to identify facilities for filing engineering data. The 3,503 numbers on this file include facilities that are proposed or demolished, numbers for facilities where the project has been canceled, and numbers to collect system or generic drawings. 
WHC-SD-SQA-CSWD-501 Rev 0

This file provided the "Hanford official Name" (FCNAME) to expand on the description of the facility. The file also provided the "Gross Square Feet" (FCSIZE) for comparison to other record size data IE the PRSIZE. It should be noted that the FCSIZE is initially input to the facility core database before design and that size may be subsequently reduced without notification to the system so any major difference between the FCSIZE \& PRSIZE fields warrants further investigation.

\section{RLPS RECORDS}

Selected fields from the property record system were added to the database by matching the property record numbers. The selected fields were contained within a download of data pertaining to Fxxxxxx, FAxxxxx, or M0xxxxx property numbered assets totaling 19,286 records.

\section{HFCR203A}

HFCR203A is a Soft Reporting table that displays the Administrator and Manager names for WHC controlled facilities. The manager name added to the database from this file is typically the manager delegated to address the day to day facility activities.

\section{HPCR442R}

HPCR442R is a Soft Reporting table that lists WHC level 2 organization codes, with the corresponding manager and organization names. This file was used to add the LEVEL2 and ORGNAME fields based on the CUSTORG assignment in the RLPS property record system.

\section{HFCR208R}

HPCR442R is the Soft Reporting "Occupied Building Table". The table was reduced to the "unique" 454 buildings which show any 0ccupant on the table. A "yes" is entered in the OCC? field where a building number match occurred. 
WHC-SD-SQA-CSWD-501 Rev 0

$\begin{array}{ll}\text { Table 1 Building Identifiers } & \begin{array}{c}\text { Appendix } C \\ \text { Data Structure }\end{array} \\ \text { Building Identifier } & \begin{array}{l}\text { General Description } \\ \text { Wood Buildings of all types }\end{array} \\ \text { S1 } & \text { Steel moment resisting frames } \\ \text { S2 } & \text { Braced steel frames } \\ \text { S3 } & \text { Light metal buildings } \\ \text { S4 } & \text { Steel frames with cast-in-place concrete shear walls } \\ \text { C1 } & \text { Concrete moment resisting frames } \\ \text { C2 } & \text { Concrete shear wall buildings } \\ \text { C3/S5 } & \text { Concrete or steel frame buildings with unreinforced } \\ \text { PC1 } & \text { masonry infill walls } \\ \text { PC2 } & \text { Tilt-up buildings } \\ \text { RM } & \text { Precast concrete frame buildings } \\ \text { URM } & \text { Reinforced masonry } \\ & \text { Unreinforced masonry }\end{array}$

Table 2 Essential Building Codes

\begin{tabular}{|l|l|}
\hline Essential Code & \multicolumn{1}{|c|}{ Description } \\
\hline Life Safety & Life Safety \\
\hline FUNCT & $\begin{array}{l}\text { Buildings which must remain functional because they are } \\
\text { required to support response to earthquake emergencies }\end{array}$ \\
\hline OPER & $\begin{array}{l}\text { Buildings which must remain operational after an earthquake } \\
\text { to maintain critical functions. }\end{array}$ \\
\hline HAZ & $\begin{array}{l}\text { Building hoising hazardous materials which if released would } \\
\text { create unacceptable risk. }\end{array}$ \\
\hline WULVAL & $\begin{array}{l}\text { Buildings housing vulnerable populations or having extremely } \\
\text { valuable contents }\end{array}$ \\
\hline None & \\
\hline
\end{tabular}


WHC-SD-SQA-CSWD-501 Rev 0

Table 3 Exemptions

\begin{tabular}{|c|c|}
\hline $\begin{array}{l}\text { Exemption } \\
\text { Code }\end{array}$ & Description \\
\hline A & $\begin{array}{l}\text { Buildings Classified for agricultural use, or intended only for } \\
\text { incidental human occupancy, or occupied by persons for a total } \\
\text { of less than } 2 \text { hours a day }\end{array}$ \\
\hline$B$ & $\begin{array}{l}\text { Detached one- and two-family dwellings that ar located in areas } \\
\text { having a boverning acceleration coefficient less than } 0.15\end{array}$ \\
\hline$C$ & $\begin{array}{l}\text { Special structure including, but not limited to: Bridges, } \\
\text { transmission towers, industrial towers and equipment, piers and } \\
\text { wharves, and hydraulic structures }\end{array}$ \\
\hline D & $\begin{array}{l}\text { One-story buildings of steel light frame or wood construction } \\
\text { with areas less than } 280 \mathrm{~m}^{2}\end{array}$ \\
\hline$E$ & $\begin{array}{l}\text { Fully-rehabilitated buildings which comply with the ICSSC RP4 } \\
\text { seismic safety standards in all four compliance categories }\end{array}$ \\
\hline$F$ & $\begin{array}{l}\text { Post-benchmark buildings as defined in Table l of ICSSC RP4 } \\
\text { which also comply with the nonstructural, geologic/site, and } \\
\text { adjacency compliance categories }\end{array}$ \\
\hline G & $\begin{array}{l}\text { Pre-benchmark buildings which have been shown by evaluation to } \\
\text { be life-safe in all four compliance categories }\end{array}$ \\
\hline H & $\begin{array}{l}\text { Buildings constructed for the Federal government whose detailed } \\
\text { design was done after the date of the adoption of Executive } \\
\text { Order } 12699 \text { and that were designed and constructed in accordance } \\
\text { with the ICSSC Guidelines and Procedures for Implementation of } \\
\text { the Executive Order on Seismic Safety of New Building } \\
\text { Construction [ICSSC 1992] }\end{array}$ \\
\hline I & $\begin{array}{l}\text { Leased buildings identified in Section } 1.3 .2 \text { (of ICSSC RP4) as } \\
\text { exempt }\end{array}$ \\
\hline $\mathrm{J}$ & $\begin{array}{l}\text { Federally permitted or regulated privately owned building on } \\
\text { Federal Land }\end{array}$ \\
\hline
\end{tabular}


WHC-SD-SQA-CSWD-501 Rev O

Table 4 Occupancy Class

\begin{tabular}{|l|l|}
\hline Occupancy Code & \\
\hline 1 & Offices \\
\hline 2 & Hospitals \\
\hline 3 & Prisons \\
\hline 4 & Schools \\
\hline 5 & Post Offices \\
\hline 6 & Other Institutions \\
\hline 7 & Housing \\
\hline 8 & Storage \\
\hline 9 & Industrial \\
\hline 10 & Service \\
\hline 11 & Research \& Development \\
\hline 12 & Miscellaneous \\
\hline
\end{tabular}


WHC-SD-SQA-CSWD-501 Rev 0

Table 5 Soit Type

\begin{tabular}{|l|l|}
\hline $\begin{array}{l}\text { Soil } \\
\text { Type }\end{array}$ & \multicolumn{1}{|c|}{ Profile with } \\
\hline S1 & $\begin{array}{l}\text { Rock of any characteristic, either shale-like or crystalline in nature. } \\
\text { Such material may be characterized by a shear wave velocity grater that }\end{array}$ \\
\hline S2 & $\begin{array}{l}\text { Deep chohesionless or stiff clay conditions including sites where the } \\
\text { soil depth exceeds } 200 \text { feet and the soil types overlying rock. }\end{array}$ \\
\hline S3 & $\begin{array}{l}\text { Soft-to-medium stiff clays and sands characterized by 30 feet or more of } \\
\text { soft or medium stiff clays with or without intervening layers of sand or }\end{array}$ \\
\hline S4 & $\begin{array}{l}\text { More than 70 feet of soft clays or silts characterized by a shear wave } \\
\text { velocity less than } 400 \text { fps. }\end{array}$ \\
\hline \hline
\end{tabular}

Table 6 Owned Building Data Structure

\begin{tabular}{|l|l|l|}
\hline Name & Type & Size \\
\hline Building & Text & 10 \\
\hline Description & Text & 30 \\
\hline County & Text & 10 \\
\hline State & Text & 10 \\
\hline Area & Text & 10 \\
\hline Gross Sq Ft & Number & Long \\
\hline Ownership & Text & 10 \\
\hline Exemption & Text & 10 \\
\hline Occupants & Number & Integer \\
\hline Occupancy Class & Number & Single \\
\hline Essential & Text & 15 \\
\hline Historic & Text & 15 \\
\hline Date of Construction & Text & 8 \\
\hline Model Building Type & Text & 5 \\
\hline Bldg Stories & Number & Integer \\
\hline Foundation Types & Text & 8 \\
\hline
\end{tabular}


WHC-SD-SQA-CSWD-501 Rev 0

\begin{tabular}{|l|l|l|}
\hline Name & Type & Size \\
\hline Risk & Text & 15 \\
\hline Evaluation Procedure & Text & 10 \\
\hline Soil Type & Text & 10 \\
\hline Evaluation Outcome & Text & 100 \\
\hline Deficient Reason & Test & 100 \\
\hline Def Struct Cost & Number & Integer \\
\hline Def NonStruct Cost & Number & Integer \\
\hline Def Finishing Cost & Number & Integer \\
\hline Def Project Cost & Number & Integer \\
\hline Def Cost Source & Text & 50 \\
\hline Perf Category & Text & 3 \\
\hline Bldg Code & Text & 10 \\
\hline Bldg Code Yr & Number & Integer \\
\hline Mission & Text & 16 \\
\hline Priority Score & Number & Integer \\
\hline Bldg Manager & Text & 15 \\
\hline Bldg Custodian & Text & 15 \\
\hline Contractor & Text & 5 \\
\hline Haz materials & Text & 3 \\
\hline Hac Category & Text & 5 \\
\hline ATC-2l Rating & Number & Double \\
\hline Restore & Yes/No & 1 \\
\hline SAR number & Text & 10 \\
\hline Struct Accidents & Text & 5 \\
\hline NPH Evaluation & Text & 4 \\
\hline & & \\
\hline & & \\
\hline
\end{tabular}




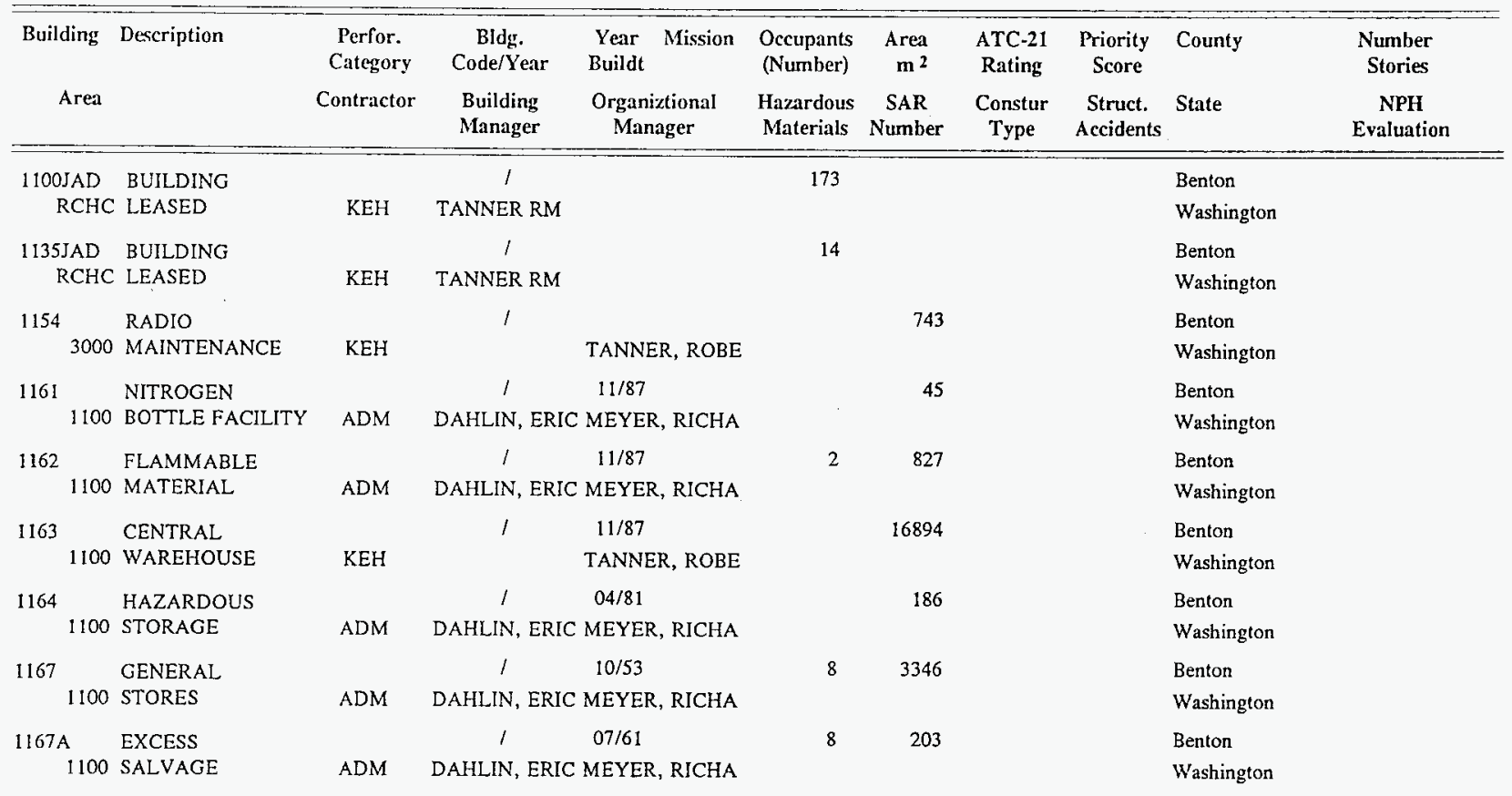


WHC-SO-SQA-CSWD-501 ReV 0

\begin{tabular}{|c|c|c|c|c|c|c|c|c|c|c|}
\hline Building & Description & $\begin{array}{c}\text { Perfor. } \\
\text { Category } \\
\text { Contractor }\end{array}$ & $\begin{array}{c}\text { Bldg. } \\
\text { Code/Year } \\
\text { Building } \\
\text { Manager }\end{array}$ & $\begin{array}{l}\text { Year Mission } \\
\text { Buildt } \\
\text { Organiztional } \\
\text { Manager }\end{array}$ & $\begin{array}{l}\text { Occupants } \\
\text { (Number) } \\
\text { Hazardous } \\
\text { Materials }\end{array}$ & $\begin{array}{c}\text { Area } \\
\text { m }^{2} \\
\text { SAR } \\
\text { Number }\end{array}$ & $\begin{array}{l}\text { ATC-21 } \\
\text { Rating } \\
\text { Constur } \\
\text { Type }\end{array}$ & $\begin{array}{l}\text { Priority } \\
\text { Score } \\
\text { Struct. } \\
\text { Accidents }\end{array}$ & State & $\begin{array}{c}\text { Number } \\
\text { Stories } \\
\text { NPH } \\
\text { Evaluation }\end{array}$ \\
\hline 1168 & CYLINDER & & 1 & $10 / 53$ & & 382 & & & Benton & \\
\hline 1100 & STORAGE & $\mathrm{ADM}$ & DAHLIN, ERIC & MEYER, RICHA & & & & & Washington & \\
\hline 1169 & CHEMICAL & & I & $07 / 61$ & & 223 & & & Benton & \\
\hline 1100 & STORAGE & ADM & DAHLIN, ERIC & MEYER, RICHA & & & & & Washington & \\
\hline 1170 & BUS TERMINAL & & 1 & $12 / 54$ & 20 & 619 & & & Benton & \\
\hline 1100 & DISPATCHERS & $\mathrm{KEH}$ & CICCONE, RIC & TANNER, ROBE & & & & & Washington & \\
\hline 1171 & MAIN & & 1 & $12 / 54$ & 129 & 8807 & & & Benton & \\
\hline 1100 & TRANSPORTATIO & $\mathrm{KEH}$ & WOODS, JAY F & TANNER, ROBE & & & & & Washington & \\
\hline $1171 \mathrm{C}$ & SPARE PARTS & & 1 & & & 93 & & & Benton & \\
\hline 1100 & STORAGE SHED & $\mathrm{KEH}$ & & & & & & & Washington & \\
\hline $1172 \mathrm{~A}$ & SERVICE STATION & & 1 & $11 / 81$ & & 28 & & & Benton & \\
\hline 1100 & & $\mathrm{KEH}$ & MEYER, RICH & MEYER, RICHA & & & & & Washington & \\
\hline 1173 & MOTOR STORES & & 1 & $12 / 54$ & & 299 & & & Benton & \\
\hline 1100 & BUILDING & $\mathrm{KEH}$ & CICCONE, RIC & TANNER, ROBE & & & & & Washington & \\
\hline 1175 & STORAGE & & 1 & $05 / 68$ & & 223 & & & Benton & \\
\hline 1100 & & $\mathrm{KEH}$ & WOODS, JAY F & TANNER, ROBE & & & & & Washington & \\
\hline 1176 & TIRE STORAGE & & 1 & $03 / 64$ & & 74 & & & Benton & \\
\hline 1100 & BUILDING & $\mathrm{KEH}$ & WOODS, JAY F & TANNER, ROBE & & & & & Washington & \\
\hline 1177 & STORAGE & & 1 & $06 / 54$ & & 71 & & & Benton & \\
\hline 1100 & BUILDING & $\mathrm{KEH}$ & WOODS, JAY F & TANNER, ROBE & & & & & Washington & \\
\hline
\end{tabular}


WHC-SD-SQA-CSWD-501 Rev 0

\begin{tabular}{|c|c|c|c|c|c|c|c|c|c|c|}
\hline Building & Description & $\begin{array}{l}\text { Perfor. } \\
\text { Category } \\
\text { Contractor }\end{array}$ & $\begin{array}{l}\text { Bldg. } \\
\text { Code/Year } \\
\text { Building } \\
\text { Manager }\end{array}$ & $\begin{array}{l}\text { Year Mission } \\
\text { Buildt } \\
\text { Organiztional } \\
\text { Manager }\end{array}$ & $\begin{array}{l}\text { Occupants } \\
\text { (Number) } \\
\text { Hazardous } \\
\text { Materials }\end{array}$ & $\begin{array}{c}\text { Area } \\
\text { m }^{2} \\
\text { SAR } \\
\text { Number }\end{array}$ & $\begin{array}{l}\text { ATC-21 } \\
\text { Rating } \\
\text { Constur } \\
\text { Type }\end{array}$ & $\begin{array}{c}\text { Priority } \\
\text { Score } \\
\text { Struct. } \\
\text { Accidents }\end{array}$ & State & $\begin{array}{c}\text { Number } \\
\text { Stories } \\
\text { NPH } \\
\text { Evaluation }\end{array}$ \\
\hline 1179 & ROAD CREW & & 1 & & & 94 & & $\cdot$ & Benton & \\
\hline 1100 & STORAGE & $\mathrm{KEH}$ & CICCONE, RIC & TANNER, ROBE & & & & & Washington & \\
\hline $1200 J \mathrm{AD}$ & BUILDING - TCPC & & 1 & & 317 & & & & Benton & \\
\hline $\mathrm{RCHC}$ & & $\mathrm{KEH}$ & TANNER RM & & & & & & Washington & \\
\hline 1205 & MOTOR FUEL & & 1 & & & 558 & & & Benton & \\
\hline 3000 & PUMP ISLANDS & KEH & & TANNER, ROBE & & & & & Washington & \\
\hline 1209 & CURRENTLY & & 1 & & & 104 & & & Benton & \\
\hline 3000 & BEING USED BY & $\mathrm{KEH}$ & & TANNER, ROBE & & & & & Washington & \\
\hline 1211 & SANDBLAST & & 1 & & & 84 & & & Benton & \\
\hline 3000 & FACILITY & $\mathrm{KEH}$ & & DIETERLE, STE & & & & & Washington & \\
\hline 1226 & HEAVY & & I & & & 876 & & & Benton & \\
\hline 3000 & EQUIPMENT & $\mathrm{KEH}$ & & TANNER, ROBE & & & & & Washington & \\
\hline 1227 & OIL STORAGE & & 1 & & & 89 & & & Benton & \\
\hline 3000 & & $\mathrm{KEH}$ & & TANNER, ROBE & & & & & Washington & \\
\hline 1235 & BOTTLED GAS & & 1 & & & 65 & & & Benton & \\
\hline 3000 & STORAGE & $\mathrm{KEH}$ & & TANNER, ROBE & & & & & Washington & \\
\hline 1240 & FABRICATION & & 1 & & & 4284 & & & Benton & \\
\hline 3000 & SHOP & $\mathrm{KEH}$ & & TANNER, ROBE & & & & & Washington & \\
\hline 1241 & PLATE SHOP & & 1 & & & 520 & & & Benton & \\
\hline 3000 & & $\mathrm{KEH}$ & & TANNER, ROBE & & & & & Washington & \\
\hline
\end{tabular}


WHC-SD-SQA-CSWD-501 ReV 0

\begin{tabular}{|c|c|c|c|c|c|c|c|c|c|c|}
\hline Building & Description & $\begin{array}{l}\text { Perfor. } \\
\text { Category } \\
\text { Contractor }\end{array}$ & $\begin{array}{l}\text { Bldg. } \\
\text { Code/Year } \\
\text { Building } \\
\text { Manager }\end{array}$ & $\begin{array}{l}\text { Year Mission } \\
\text { Buildt } \\
\text { Organiztional } \\
\text { Manager }\end{array}$ & $\begin{array}{l}\text { Occupants } \\
\text { (Number) } \\
\text { Hazardous } \\
\text { Materials }\end{array}$ & $\begin{array}{c}\text { Area } \\
\mathrm{m}^{2} \\
\text { SAR } \\
\text { Number }\end{array}$ & $\begin{array}{l}\text { ATC-21 } \\
\text { Rating } \\
\text { Constur } \\
\text { Type }\end{array}$ & $\begin{array}{l}\text { Priority } \\
\text { Score } \\
\text { Struct. } \\
\text { Accidents }\end{array}$ & State & $\begin{array}{c}\text { Number } \\
\text { Stories } \\
\text { NPH } \\
\text { Evaluation }\end{array}$ \\
\hline 1242 & COMPRESSOR & & 1 & & & 89 & & & Benton & \\
\hline 3000 & SHOP & $\mathrm{KEH}$ & & TANNER, ROBE & & & & & Washington & \\
\hline 1252 & WAREHOUSE & & $I$ & & & 1366 & & & Benton & \\
\hline 3000 & AND SAFETY & $\mathrm{KEH}$ & & TANNER, ROBE & & & & & Washington & \\
\hline 1253 & COMBUSTIBLE & & 1 & & & 124 & & & Benton & \\
\hline 3000 & MATERIAL & $\mathrm{KEH}$ & & TANNER, ROBE & & & & & Washington & \\
\hline 1256 & ADMINISTRATION & & 1 & & & 942 & & & Benton & \\
\hline 3000 & BUILDING & $\mathrm{KEH}$ & & TANNER, ROBE & & & & & Washington & \\
\hline 1262 & ADMINISTRATION & & I & & & 565 & & & Benton & \\
\hline 3000 & AND & $\mathrm{KEH}$ & & TANNER, ROBE & & & & & Washington & \\
\hline 1264 & OFFICE BUILDING & & 1 & & & 836 & & & Benton & \\
\hline 3000 & & $\mathrm{KEH}$ & & TANNER, ROBE & & & & & Washington & \\
\hline $1506 \mathrm{~K} 1$ & FIBER OPTICS & & 1 & & & 48 & & & Benton & \\
\hline $100 \mathrm{~K}$ & COMPUTER HUT & $\mathrm{BCS}$ & & & & & & & Washington & \\
\hline $150 \mathrm{KE}$ & HEAT RECOVERY & & 1 & $03 / 55$ & & 88 & & & Benton & \\
\hline $100 K$ & FACILITY & SNF & & SIDDOWAY, DE & & & & & Washington & \\
\hline $150 \mathrm{KW}$ & HEAT RECOVERY & & 1 & $03 / 55$ & & 88 & & & Benton & \\
\hline $100 K$ & FACILITY & SNF & & SIDDOWAY, DE & & & & & Washington & \\
\hline $1512 \mathrm{~N}$ & GAS BOTTLE & & 1 & & & 19 & & & Benton & \\
\hline $100 \mathrm{~N}$ & STORAGE & $\mathrm{KEH}$ & & WITHERSPOON, & & & & & Washington & \\
\hline
\end{tabular}


WHC-SD-SQA-CSWD-501 Rev 0

\begin{tabular}{|c|c|c|c|c|c|c|c|c|c|c|}
\hline Building & Description & $\begin{array}{l}\text { Perfor. } \\
\text { Category }\end{array}$ & $\begin{array}{l}\text { Bldg. } \\
\text { Code/Year }\end{array}$ & $\begin{array}{l}\text { Year Mission } \\
\text { Buildt }\end{array}$ & $\begin{array}{l}\text { Occupants } \\
\text { (Number) }\end{array}$ & $\begin{array}{r}\text { Area } \\
\mathrm{m}^{2}\end{array}$ & $\begin{array}{c}\text { ATC-21 } \\
\text { Rating }\end{array}$ & $\begin{array}{l}\text { Priority } \\
\text { Score }\end{array}$ & County & $\begin{array}{c}\text { Number } \\
\text { Stories }\end{array}$ \\
\hline Area & & Contractor & $\begin{array}{l}\text { Building } \\
\text { Manager }\end{array}$ & $\begin{array}{l}\text { Organiztional } \\
\text { Manager }\end{array}$ & $\begin{array}{c}\text { Hazardous } \\
\text { Materials }\end{array}$ & $\begin{array}{c}\text { SAR } \\
\text { Number }\end{array}$ & $\begin{array}{l}\text { Constur } \\
\text { Type }\end{array}$ & $\begin{array}{l}\text { Struct. } \\
\text { Accidents }\end{array}$ & State & $\begin{array}{c}\text { NPH } \\
\text { Evaluation }\end{array}$ \\
\hline $\begin{array}{l}1515 \mathrm{~N} \\
100 \mathrm{~N}\end{array}$ & $\begin{array}{l}\text { MULTICRAFT } \\
\text { SHOP }\end{array}$ & $\mathrm{KEH}$ & $\begin{array}{c}\text { / } \\
\text { WITHERSPOO }\end{array}$ & $\begin{array}{l}02 / 83 \\
\text { WITHERSPOON, }\end{array}$ & & 446 & & & $\begin{array}{l}\text { Benton } \\
\text { Washington }\end{array}$ & \\
\hline $\begin{array}{l}1516 \mathrm{~N} \\
100 \mathrm{~N}\end{array}$ & $\begin{array}{l}\text { CARPENTER } \\
\text { SHOP }\end{array}$ & $\mathrm{KEH}$ & $\begin{array}{c}l \\
\text { WITHERSPOO }\end{array}$ & WITHERSPOON, & & 45 & & & $\begin{array}{l}\text { Benton } \\
\text { Washington }\end{array}$ & \\
\hline $\begin{array}{r}1517 \mathrm{~N} \\
100 \mathrm{~N}\end{array}$ & $\begin{array}{l}\text { PAINT } \\
\text { SHOP/LABORER }\end{array}$ & $\mathrm{KEH}$ & $\begin{array}{c}l \\
\text { WITHERSPOO }\end{array}$ & WITHERSPOON, & & 74 & & & $\begin{array}{l}\text { Benton } \\
\text { Washington }\end{array}$ & \\
\hline $\begin{array}{l}1518 \mathrm{~N} \\
100 \mathrm{~N}\end{array}$ & $\begin{array}{l}\text { ELECTRICAL } \\
\text { SHOP }\end{array}$ & $\mathrm{KEH}$ & $\begin{array}{c}/ \\
\text { MARTIN, RIC }\end{array}$ & WITHERSPOON, & & 74 & & & $\begin{array}{l}\text { Benton } \\
\text { Washington }\end{array}$ & \\
\hline $\begin{array}{l}1519 \mathrm{~N} \\
100 \mathrm{~N}\end{array}$ & FITTERS SHOP & $\mathrm{KEH}$ & $\begin{array}{c}\prime \\
\text { STAIR, JEFFRE }\end{array}$ & WITHERSPOON, & & 74 & & & $\begin{array}{l}\text { Benton } \\
\text { Washington }\end{array}$ & \\
\hline $151 \mathrm{~B}$ & $\begin{array}{l}\text { PRIMARY } \\
\text { SUBSTATION }\end{array}$ & $\mathrm{KEH}$ & $\begin{array}{c}\text { I } \\
\text { FRITZ, ROBER }\end{array}$ & $\begin{array}{l}12 / 44 \\
\text { DEBBAN, HERB }\end{array}$ & & 610 & & & $\begin{array}{l}\text { Benton } \\
\text { Washington }\end{array}$ & \\
\hline $\begin{array}{l}1510 \\
100 D\end{array}$ & $\begin{array}{l}\text { PRIMARY } \\
\text { SUBSTATION }\end{array}$ & KEH & $\begin{array}{c}\prime \\
\text { FRITZ, ROBER }\end{array}$ & $\begin{array}{l}12 / 44 \\
\text { DEBBAN, HERB }\end{array}$ & & 550 & & & $\begin{array}{l}\text { Benton } \\
\text { Washington }\end{array}$ & \\
\hline $\begin{array}{l}1614 \mathrm{~K} 3 \\
100 \mathrm{~K}\end{array}$ & $\begin{array}{l}\text { ENVIRONMENTA } \\
\text { L MONITORING }\end{array}$ & SNF & $\begin{array}{c}/ \\
\text { THOMPSON, C }\end{array}$ & $\begin{array}{l}\text { 04/55 } \\
\text { SIDDOWAY, DE }\end{array}$ & & 6 & & & $\begin{array}{l}\text { Benton } \\
\text { Washington }\end{array}$ & \\
\hline $\begin{array}{l}165 \mathrm{KE} \\
100 \mathrm{~K}\end{array}$ & $\begin{array}{l}\text { POWER } \\
\text { CONTROL }\end{array}$ & SNF & $\begin{array}{c}\text { / } \\
\text { MILLER, CHA }\end{array}$ & $\begin{array}{l}\text { 03/55 } \\
\text { SIDDOWAY, DE }\end{array}$ & & 4907 & & & $\begin{array}{l}\text { Benton } \\
\text { Washington }\end{array}$ & \\
\hline $\begin{array}{l}1705 \mathrm{KE} \\
100 \mathrm{~K}\end{array}$ & $\begin{array}{l}\text { EFFLUENT } \\
\text { WATER }\end{array}$ & SNF & $\begin{array}{c}\text { / } \\
\text { MILLER, CHA }\end{array}$ & $\begin{array}{l}\text { 07/62 } \\
\text { SIDDOWAY, DE }\end{array}$ & & 51 & & & $\begin{array}{l}\text { Benton } \\
\text { Washington }\end{array}$ & \\
\hline
\end{tabular}


WHC-SD-SQA-CSWD-501 Rev 0

\begin{tabular}{|c|c|c|c|c|c|c|c|c|c|c|}
\hline Building & Description & $\begin{array}{c}\text { Perfor. } \\
\text { Category } \\
\text { Contractor }\end{array}$ & $\begin{array}{l}\text { Bldg. } \\
\text { Code/Year } \\
\text { Building } \\
\text { Manager }\end{array}$ & $\begin{array}{l}\text { Year Mission } \\
\text { Buildt } \\
\text { Organiztional } \\
\text { Manager }\end{array}$ & $\begin{array}{l}\text { Occupants } \\
\text { (Number) } \\
\text { Hazardous } \\
\text { Materials }\end{array}$ & $\begin{array}{c}\text { Area } \\
\text { m }^{2} \\
\text { SAR } \\
\text { Number }\end{array}$ & $\begin{array}{l}\text { ATC-2I } \\
\text { Rating } \\
\text { Constur } \\
\text { Type }\end{array}$ & $\begin{array}{l}\text { Priority } \\
\text { Score } \\
\text { Struct. } \\
\text { Accidents }\end{array}$ & State & $\begin{array}{c}\text { Number } \\
\text { Stories } \\
\text { NPH } \\
\text { Evaluation }\end{array}$ \\
\hline $1706 \mathrm{KE}$ & WATER STUDIES & & 1 & $03 / 55$ & 3 & 1041 & & & Benton & \\
\hline $100 \mathrm{~K}$ & SEMIWORKS & TWR & THOMPSON, C & ERMOLD, LEON & & & & & Washington & \\
\hline $1706 \mathrm{KEL}$ & DEVELOPMENT & & 1 & $04 / 63$ & & 625 & & & Benton & \\
\hline $100 \mathrm{~K}$ & LABORATORY & TWR & THOMPSON, C & ERMOLD, LEON & & & & & Washington & \\
\hline 1706KER & WATER STUDIES & & 1 & $03 / 55$ & & 1074 & & & Benton & \\
\hline $100 \mathrm{~K}$ & RECIRCULATION & TWR & THOMPSON, C & ERMOLD, LEON & & & & & Washington & \\
\hline $1713 \mathrm{KE}$ & SHOP BUILDING & & 1 & $03 / 49$ & & 74 & & & Benton & \\
\hline $100 \mathrm{~K}$ & & SNF & MILLER, CHA & SIDDOWAY, DE & & & & & Washington & \\
\hline $1713 \mathrm{KW}$ & WAREHOUSE & & 1 & $03 / 49$ & & 28 & & & Benton & \\
\hline $100 \mathrm{~K}$ & & SNF & MIILER, CHA & SIDDOWAY, DE & & & & & Washington & \\
\hline $1714 \mathrm{KE}$ & OIL AND PAINT & & 1 & $03 / 55$ & & 18 & & & Benton & \\
\hline $100 \mathrm{~K}$ & STORAGE SHED & SNF & MILLER, CHA & SIDDOWAY, DE & & & & & Washington & \\
\hline $1717 \mathrm{~K}$ & & & 1 & $03 / 55$ & & & & & Benton & \\
\hline $100 \mathrm{~K}$ & & SNF & & SIDDOWAY, DE & & & & & Washington & \\
\hline $1720 \mathrm{~K}$ & OFFICES AND & & 1 & $03 / 55$ & & 344 & & & Benton & \\
\hline $100 \mathrm{~K}$ & TELEPHONE & SNF & MILLER, CHA & SIDDOWAY, DE & & & & & Washington & \\
\hline $1802 \mathrm{TD}$ & BUILDING - & & 1 & & 29 & & & & Benton & \\
\hline $\mathrm{RCHC}$ & LEASED & $\mathrm{KEH}$ & TANNER RM & & & & & & Washington & \\
\hline $1804 \mathrm{TD}$ & BUILDING - & & 1 & & 20 & & & & Benton & \\
\hline $\mathrm{RCHC}$ & LEASED & $\mathrm{KEH}$ & TANNER RM & & & & & & Washington & \\
\hline
\end{tabular}


WHC.SD.SQA-CSWD-501 Rev 0

\begin{tabular}{|c|c|c|c|c|c|c|c|c|c|c|}
\hline Area & Description & $\begin{array}{c}\text { Perfor. } \\
\text { Category } \\
\text { Contractor }\end{array}$ & $\begin{array}{l}\text { Bldg. } \\
\text { Code/Year } \\
\text { Building } \\
\text { Manager }\end{array}$ & $\begin{array}{l}\text { Year Mission } \\
\text { Buildt } \\
\text { Organiztional } \\
\text { Manager }\end{array}$ & $\begin{array}{c}\text { Occupants } \\
\text { (Number) } \\
\text { Hazardous } \\
\text { Materials }\end{array}$ & $\begin{array}{c}\text { Area } \\
\text { m }^{2} \\
\text { SAR } \\
\text { Number }\end{array}$ & $\begin{array}{c}\text { ATC-21 } \\
\text { Rating } \\
\text { Constur } \\
\text { Type }\end{array}$ & $\begin{array}{l}\text { Priority } \\
\text { Score } \\
\text { Struct. } \\
\text { Accidents }\end{array}$ & State & $\begin{array}{c}\text { Number } \\
\text { Stories } \\
\text { NPH } \\
\text { Evaluation }\end{array}$ \\
\hline $\begin{array}{l}\text { 1806TD } \\
\text { RCHC }\end{array}$ & $\begin{array}{l}\text { BUILDING - } \\
\text { LEASED }\end{array}$ & $\mathrm{KEH}$ & $\begin{array}{c}\prime \\
\text { TANNER RM }\end{array}$ & & 29 & & & & $\begin{array}{l}\text { Benton } \\
\text { Washington }\end{array}$ & \\
\hline $\begin{array}{l}1808 \mathrm{TD} \\
\text { RCHC }\end{array}$ & $\begin{array}{l}\text { BUILDING - } \\
\text { LEASED }\end{array}$ & KEH & $\begin{array}{c}\prime \\
\text { TANNER RM }\end{array}$ & & 16 & & & & $\begin{array}{l}\text { Benton } \\
\text { Washington }\end{array}$ & \\
\hline $\begin{array}{l}1810 \mathrm{TD} \\
\mathrm{RCHC}\end{array}$ & $\begin{array}{l}\text { BUILDING - } \\
\text { LEASED }\end{array}$ & $\mathrm{KEH}$ & $\begin{array}{c}\prime \\
\text { TANNER RM }\end{array}$ & & 3 & & & & $\begin{array}{l}\text { Benton } \\
\text { Washington }\end{array}$ & \\
\hline $\begin{array}{l}\text { 1812TD } \\
\text { RCHC }\end{array}$ & $\begin{array}{l}\text { BUILDING - } \\
\text { LEASED }\end{array}$ & $\mathrm{KEH}$ & $\begin{array}{c}\prime \\
\text { TANNER RM }\end{array}$ & & 1 & & & & $\begin{array}{l}\text { Benton } \\
\text { Washington }\end{array}$ & \\
\hline $\begin{array}{l}\text { 1814TD } \\
\text { RCHC }\end{array}$ & $\begin{array}{l}\text { BUILDING - } \\
\text { LEASED }\end{array}$ & $\mathrm{KEH}$ & $\begin{array}{c}/ \\
\text { TANNER RM }\end{array}$ & & 2 & & & & $\begin{array}{l}\text { Benton } \\
\text { Washington }\end{array}$ & \\
\hline $\begin{array}{l}1816 \mathrm{TD} \\
\mathrm{RCHC}\end{array}$ & $\begin{array}{l}\text { BUILDING - } \\
\text { LEASED }\end{array}$ & $\mathrm{KEH}$ & $\begin{array}{c}/ \\
\text { TANNER RM }\end{array}$ & & & & & & $\begin{array}{l}\text { Benton } \\
\text { Washington }\end{array}$ & \\
\hline $\begin{array}{l}\text { 1818TD } \\
\text { RCHC }\end{array}$ & $\begin{array}{l}\text { BUILDING - } \\
\text { LEASED }\end{array}$ & $\mathrm{KEH}$ & $\begin{array}{c}I \\
\text { TANNER RM }\end{array}$ & & 4 & & & & $\begin{array}{l}\text { Benton } \\
\text { Washington }\end{array}$ & \\
\hline $181 \mathrm{~B}$ & $\begin{array}{l}\text { RIVER PUMP } \\
\text { HOUSE, SERVES }\end{array}$ & $\mathrm{KEH}$ & $\begin{array}{c}/ \\
\text { DEBBAN, HER }\end{array}$ & $\begin{array}{l}12 / 44 \\
\text { DEBBAN, HERB }\end{array}$ & & 1183 & & & $\begin{array}{l}\text { Benton } \\
\text { Washington }\end{array}$ & \\
\hline $\begin{array}{l}181 \mathrm{D} \\
100 \mathrm{D}\end{array}$ & $\begin{array}{l}\text { RIVER PUMP } \\
\text { HOUSE, SERVES }\end{array}$ & KEH & $\begin{array}{c}/ \\
\text { DEBBAN, HER }\end{array}$ & $\begin{array}{l}12 / 44 \\
\text { DEBBAN, HERB }\end{array}$ & & 796 & & & $\begin{array}{l}\text { Benton } \\
\text { Washington }\end{array}$ & \\
\hline $\begin{array}{l}18 \mathrm{IKE} \\
100 \mathrm{~K}\end{array}$ & $\begin{array}{l}\text { RIVER PUMP } \\
\text { HOUSE }\end{array}$ & SNF & 1 & $\begin{array}{l}03 / 55 \\
\text { SIDDOWAY, DE }\end{array}$ & & & & & $\begin{array}{l}\text { Benton } \\
\text { Washington }\end{array}$ & \\
\hline
\end{tabular}


WHC.SD-SQA-CSWD-501 Rev 0

\begin{tabular}{|c|c|c|c|c|c|c|c|c|c|c|}
\hline Building & Description & $\begin{array}{c}\text { Perfor. } \\
\text { Category } \\
\text { Contractor }\end{array}$ & $\begin{array}{l}\text { Bldg. } \\
\text { Code/Year } \\
\text { Building } \\
\text { Manager }\end{array}$ & $\begin{array}{l}\text { Year Mission } \\
\text { Buildt } \\
\text { Organiztional } \\
\text { Manager }\end{array}$ & $\begin{array}{l}\text { Occupants } \\
\text { (Number) } \\
\text { Hazardous } \\
\text { Materials }\end{array}$ & $\begin{array}{c}\text { Area } \\
\text { m }^{2} \\
\text { SAR } \\
\text { Number }\end{array}$ & $\begin{array}{l}\text { ATC-21 } \\
\text { Rating } \\
\text { Constur } \\
\text { Type }\end{array}$ & $\begin{array}{l}\text { Priority } \\
\text { Score } \\
\text { Struct. } \\
\text { Accidents }\end{array}$ & State & $\begin{array}{c}\text { Number } \\
\text { Stories } \\
\text { NPH } \\
\text { Evaluation }\end{array}$ \\
\hline $1820 T D$ & BUILDING & & 1 & & 18 & & & & Benton & \\
\hline $\mathrm{RCHC}$ & LEASED & $\mathrm{KEH}$ & TANNER RM & & & & & & Washington & \\
\hline $182 \mathrm{~B}$ & RESERVOIR AND & & 1 & $12 / 44$ & 4 & 1366 & & & Benton & \\
\hline $100 \mathrm{~B}$ & PUMP HOUSE & $\mathrm{KEH}$ & DEBBAN, HER & DEBBAN, HERB & & & & & Washington & \\
\hline $182 \mathrm{D}$ & RESERVOIR AND & & 1 & $12 / 44$ & & 1366 & & & Benton & \\
\hline $100 \mathrm{D}$ & PUMP HOUSE & KEH & DEBBAN, HER & DEBBAN, HERB & & & & & Washington & \\
\hline $\begin{array}{r}183.1 \mathrm{KE} \\
100 \mathrm{~K}\end{array}$ & $\begin{array}{l}\text { HEADHOUSE/CHL } \\
\text { ORINE }\end{array}$ & SNF & 1 & $\begin{array}{l}03 / 55 \\
\text { SIDDOWAY, DE }\end{array}$ & & 34 & & & $\begin{array}{l}\text { Benton } \\
\text { Washington }\end{array}$ & \\
\hline $\begin{array}{r}183.1 \mathrm{KW} \\
100 \mathrm{~K}\end{array}$ & $\begin{array}{l}\text { HEADHOUSE/CHL } \\
\text { ORINE }\end{array}$ & SNF & 1 & $\begin{array}{l}\text { 03/55 } \\
\text { SIDDOWAY, DE }\end{array}$ & & 34 & & & $\begin{array}{l}\text { Benton } \\
\text { Washington }\end{array}$ & \\
\hline $\begin{array}{r}183.5 \mathrm{KE} \\
\text { I0OK }\end{array}$ & $\begin{array}{l}\text { LIME FEEDER } \\
\text { BUILDING }\end{array}$ & SNF & 1 & $\begin{array}{l}\text { 03/55 } \\
\text { SIDDOWAY, DE }\end{array}$ & & 86 & & & $\begin{array}{l}\text { Benton } \\
\text { Washington }\end{array}$ & \\
\hline $\begin{array}{r}183.6 \mathrm{KE} \\
100 \mathrm{~K}\end{array}$ & $\begin{array}{l}\text { LIME FEEDER } \\
\text { BUILDING }\end{array}$ & SNF & 1 & $\begin{array}{l}\text { 03/55 } \\
\text { SIDDOWAY, DE }\end{array}$ & & 86 & & & $\begin{array}{l}\text { Benton } \\
\text { Washington }\end{array}$ & \\
\hline $\begin{array}{r}183.6 \mathrm{KW} \\
100 \mathrm{~K}\end{array}$ & $\begin{array}{l}\text { LIME FEEDER } \\
\text { BUILDING }\end{array}$ & SNF & 1 & $\begin{array}{l}\text { 03/55 } \\
\text { SIDDOWAY, DE }\end{array}$ & & 86 & & & $\begin{array}{l}\text { Benton } \\
\text { Washington }\end{array}$ & \\
\hline $183 \mathrm{D}$ & $\begin{array}{l}\text { FILTER PLANT } \\
\text { POWER }\end{array}$ & $\mathrm{KEH}$ & $\begin{array}{c}/ \\
\text { DEBBAN, HER }\end{array}$ & $\begin{array}{l}\text { 12/44 } \\
\text { DEBBAN, HERB }\end{array}$ & 4 & 6055 & & & $\begin{array}{l}\text { Benton } \\
\text { Washington }\end{array}$ & \\
\hline $\begin{array}{l}183 \mathrm{KE} \\
100 \mathrm{~K}\end{array}$ & $\begin{array}{l}\text { FILTER PLANT } \\
\text { HEAD HOUSE, }\end{array}$ & SNF & $\begin{array}{c}/ \\
\text { MILLER, CHA }\end{array}$ & $\begin{array}{l}\text { 03/55 } \\
\text { SIDDOWAY, DE }\end{array}$ & 4 & 1741 & & & $\begin{array}{l}\text { Benton } \\
\text { Washington }\end{array}$ & \\
\hline
\end{tabular}


WHC-SD-SQA-CSWD-501 Rev 0

\begin{tabular}{|c|c|c|c|c|c|c|c|c|c|c|}
\hline Area & Description & $\begin{array}{c}\text { Perfor. } \\
\text { Category } \\
\text { Contractor }\end{array}$ & $\begin{array}{c}\text { Bldg. } \\
\text { Code/Year } \\
\text { Building } \\
\text { Manager }\end{array}$ & $\begin{array}{l}\text { Year Mission } \\
\text { Buildt } \\
\text { Organiztional } \\
\text { Manager }\end{array}$ & $\begin{array}{l}\text { Occupants } \\
\text { (Number) } \\
\text { Hazardous } \\
\text { Materials }\end{array}$ & $\begin{array}{c}\text { Area } \\
m^{2} \\
\text { SAR } \\
\text { Number }\end{array}$ & $\begin{array}{l}\text { ATC-21 } \\
\text { Rating } \\
\text { Constur } \\
\text { Type }\end{array}$ & $\begin{array}{l}\text { Priority } \\
\text { Score } \\
\text { Struct. } \\
\text { Accidents }\end{array}$ & State & $\begin{array}{c}\text { Number } \\
\text { Stories } \\
\text { NPH } \\
\text { Evaluation }\end{array}$ \\
\hline $1901 Y$ & & & 1 & & & & & & Benton & \\
\hline 600 & & $\mathrm{KEH}$ & & & & & & & Washington & \\
\hline $1908 \mathrm{KE}$ & EFFLUENT & & 1 & $04 / 55$ & & 14 & & & Benton & \\
\hline $100 \mathrm{~K}$ & WATER & SNF & MILLER, CHA & SIDDOWAY, DE & & & & & Washington & \\
\hline $\begin{array}{l}190 \mathrm{KE} \\
100 \mathrm{~K}\end{array}$ & $\begin{array}{l}\text { MAIN PUMP } \\
\text { HOUSE }\end{array}$ & SNF & $\begin{array}{c}/ \\
\text { EBBESON, LE }\end{array}$ & $\begin{array}{l}\text { 03/55 } \\
\text { SIDDOWAY, DE }\end{array}$ & 2 & 4427 & & & $\begin{array}{l}\text { Benton } \\
\text { Washington }\end{array}$ & \\
\hline $\begin{array}{l}1979 \text { SNY } \\
\text { RCHN }\end{array}$ & 1979 SNYDER & $\mathrm{KEH}$ & $\begin{array}{c}\prime \\
\text { TANNER RM }\end{array}$ & & 48 & & & & $\begin{array}{l}\text { Benton } \\
\text { Washington }\end{array}$ & \\
\hline $\begin{array}{l}\text { 1981SNY } \\
\text { RCHN }\end{array}$ & 1981 SNYDER & $\mathrm{KEH}$ & $\begin{array}{c}\prime \\
\text { TANNER RM }\end{array}$ & & 1 & & & & $\begin{array}{l}\text { Benton } \\
\text { Washington }\end{array}$ & \\
\hline $\begin{array}{l}201 \mathrm{~W} \\
200 \mathrm{~W}\end{array}$ & $\begin{array}{l}\text { INSTRUMENT } \\
\text { BUILDING SE }\end{array}$ & PSS & $\begin{array}{c}/ \\
\text { LOONEY, CLA }\end{array}$ & DILIBERTO, AN & & 36 & & & $\begin{array}{l}\text { Benton } \\
\text { Washington }\end{array}$ & \\
\hline $\begin{array}{l}2025 \mathrm{E} \\
200 \mathrm{E}\end{array}$ & $\begin{array}{l}\text { EFFLUENT } \\
\text { TREATMENT }\end{array}$ & PSS & $\begin{array}{c}\text { / } \\
\text { WURZ, RICHA }\end{array}$ & DILIBERTO, AN & 53 & 3717 & & & $\begin{array}{l}\text { Benton } \\
\text { Washington }\end{array}$ & \\
\hline $\begin{array}{l}2025 E A \\
200 E\end{array}$ & $\begin{array}{l}\text { EFFLUENT } \\
\text { TREATMENT }\end{array}$ & $\mathrm{KEH}$ & $\begin{array}{c}\text { I } \\
\text { GONZALES, RI }\end{array}$ & $\begin{array}{l}12 / 94 \\
\text { DILIBERTO, AN }\end{array}$ & 47 & 1208 & & & $\begin{array}{l}\text { Benton } \\
\text { Washington }\end{array}$ & \\
\hline $\begin{array}{r}2025 \mathrm{EC} 71 \\
200 \mathrm{E}\end{array}$ & $\begin{array}{l}\text { ETF LCU } \\
\text { BUILDING }\end{array}$ & PSS & 1 & $\begin{array}{l}06 / 95 \\
\text { DILIBERTO, AN }\end{array}$ & & 11 & & & $\begin{array}{l}\text { Benton } \\
\text { Washington }\end{array}$ & \\
\hline $\begin{array}{l}202 \mathrm{~A} \\
200 \mathrm{E}\end{array}$ & $\begin{array}{l}\text { PUREX CANYON } \\
\text { AND SERVICE }\end{array}$ & TRP & $\begin{array}{c}/ \\
\text { HAMRICK, DO }\end{array}$ & $\begin{array}{l}12 / 55 \\
\text { HAMRICK, DOU }\end{array}$ & 47 & 17555 & & & $\begin{array}{l}\text { Benton } \\
\text { Washington }\end{array}$ & \\
\hline
\end{tabular}


WHC-SD-SQA-CSWD-501 Rev 0

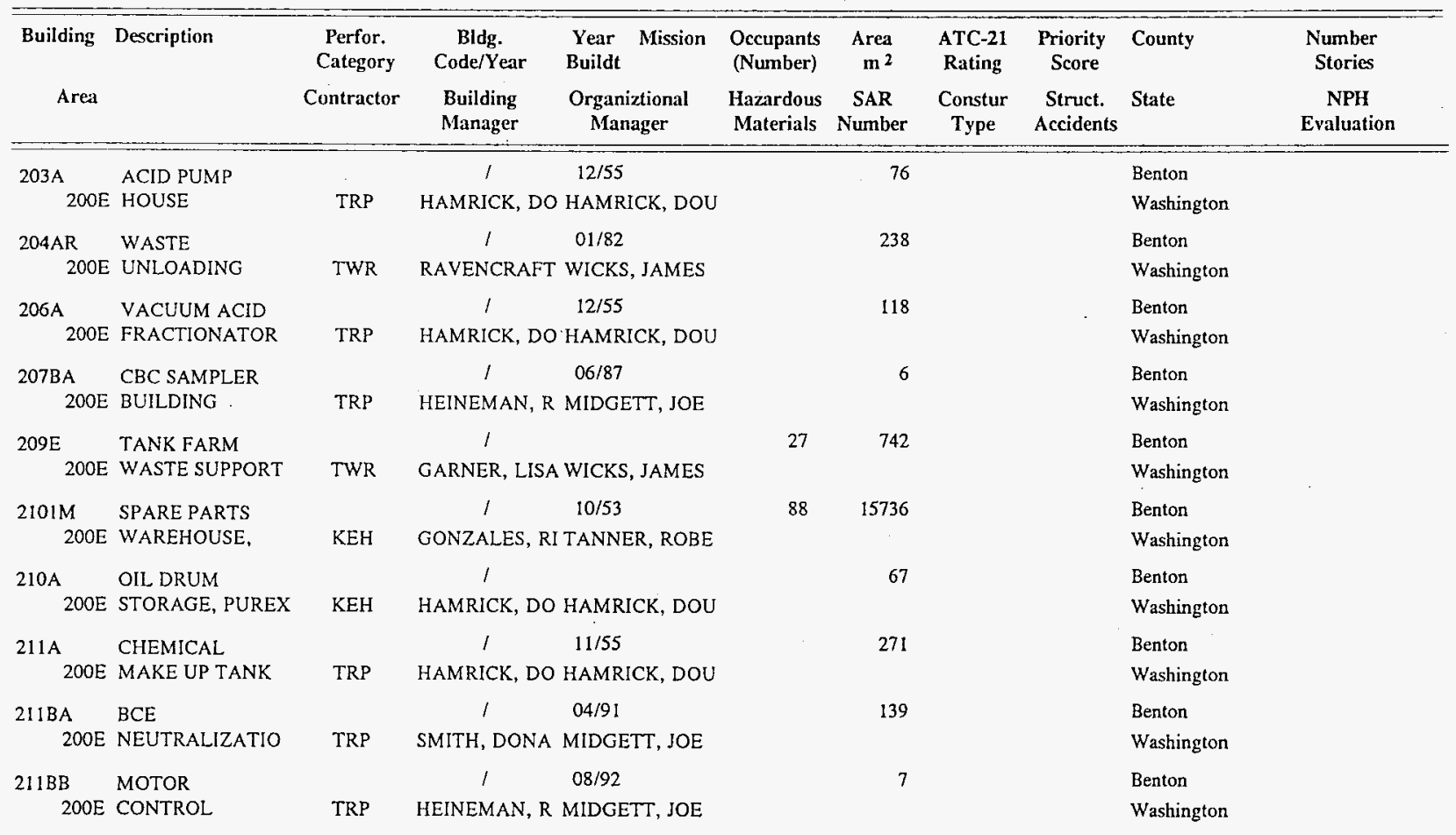


WHC-SD-SQA-CSWD-501 Rev 0

\begin{tabular}{|c|c|c|c|c|c|c|c|c|c|c|}
\hline Building & Description & $\begin{array}{l}\text { Perfor. } \\
\text { Category }\end{array}$ & $\begin{array}{c}\text { Bldg. } \\
\text { Code/Year }\end{array}$ & $\begin{array}{c}\text { Year Mission } \\
\text { Buildt }\end{array}$ & $\begin{array}{l}\text { Occupants } \\
\text { (Number) }\end{array}$ & $\begin{array}{r}\text { Area } \\
\mathrm{m}^{2}\end{array}$ & $\begin{array}{l}\text { ATC-2l } \\
\text { Rating }\end{array}$ & $\begin{array}{c}\text { Priority } \\
\text { Score }\end{array}$ & County & $\begin{array}{c}\text { Number } \\
\text { Stories }\end{array}$ \\
\hline Area & & Contractor & $\begin{array}{l}\text { Building } \\
\text { Manager }\end{array}$ & $\begin{array}{c}\text { Organiztional } \\
\text { Manager }\end{array}$ & $\begin{array}{l}\text { Hazardous } \\
\text { Materials }\end{array}$ & $\begin{array}{c}\text { SAR } \\
\text { Number }\end{array}$ & $\begin{array}{l}\text { Constur } \\
\text { Type }\end{array}$ & $\begin{array}{l}\text { Struct. } \\
\text { Accidents }\end{array}$ & State & $\begin{array}{c}\text { NPH } \\
\text { Evaluation }\end{array}$ \\
\hline $211 \mathrm{~T} 52$ & $211 \mathrm{~T}$ & & 1 & $07 / 90$ & & 13 & & & Benton & \\
\hline $200 W$ & INSTRUMENT & PSS & & HAMILTON, WI & & & & & Washington & \\
\hline $212 \mathrm{~A}$ & FISSION & & 1 & $03 / 60$ & & 36 & & & Benton & \\
\hline $200 \mathrm{E}$ & PRODUCT LOAD & TRP & HAMRICK, DO & HAMRICK, DOU & & & & & Washington & \\
\hline $212 S$ & COVERED GAS & & 1 & $05 / 91$ & & 79 & & & Benton & \\
\hline $200 \mathrm{~W}$ & CYL STORAGE & TWR & & ERMOLD, LEON & & & & & Washington & \\
\hline $213 A$ & FISSION & & 1 & $01 / 61$ & & 48 & & & Benton & \\
\hline $200 \mathrm{E}$ & PRODUCT LOAD & TRP & HAMRICK, DO & HAMRICK, DOU & & & & & Washington & \\
\hline $213 \mathrm{~K}$ & SODIUM & & 1 & & & & & & Benton & \\
\hline 600 & STORAGE & ADM & DAHLIN, ERIC & MEYER, RICHA & & & & & Washington & \\
\hline $213 W$ & WASTE & & 1 & $08 / 85$ & & 80 & & & Benton & \\
\hline $200 \mathrm{~W}$ & COMPACTOR & TWR & REBER, DAVI & WICKS, JAMES & & & & & Washington & \\
\hline $214 \mathrm{~A}$ & PUREX & & 1 & $03 / 86$ & & 94 & & & Benton & \\
\hline $200 E$ & WAREHOUSE & TRP & HAMRICK, DO & HAMRICK, DOU & & & & & Washington & \\
\hline $214 \mathrm{~T}$ & CHEMCIAL & & 1 & $09 / 86$ & & 143 & & & Benton & \\
\hline $200 \mathrm{~W}$ & STORAGE & PSS & WRIGHT, MAL & HAMILTON, WI & & & & & Washington & \\
\hline 216A271 & VALVE CONTROL & & 1 & $01 / 58$ & & 9 & & & Benton & \\
\hline $200 E$ & HOUSE & TWR & SPARKS, DAVI & WICKS, JAMES & & & & & Washington & \\
\hline $21629 B$ & OPERATOR'S & & 1 & $12 / 75$ & & 12 & & & Benton & \\
\hline $200 W$ & CUBICLE 21629 & PSS & BURKS, DAVI & VOGT, ERIC C & & & & & Washington & \\
\hline
\end{tabular}


WHC.SD-SQA-CSWD-50I Rev 0

\begin{tabular}{|c|c|c|c|c|c|c|c|c|c|c|}
\hline \multirow{2}{*}{$\begin{array}{r}\text { Building } \\
\text { Area }\end{array}$} & \multirow[t]{2}{*}{ Description } & \multirow{2}{*}{$\begin{array}{c}\text { Perfor. } \\
\text { Category } \\
\text { Contractor }\end{array}$} & \multirow{2}{*}{$\begin{array}{c}\text { Bldg. } \\
\text { Code/Year } \\
\text { Building } \\
\text { Manager }\end{array}$} & \multirow{2}{*}{$\begin{array}{l}\text { Year Mission } \\
\text { Buildt } \\
\text { Organiztional } \\
\text { Manager }\end{array}$} & \multirow{2}{*}{$\begin{array}{c}\text { Occupants } \\
\text { (Number) } \\
\text { Hazardous } \\
\text { Materials }\end{array}$} & \multirow{2}{*}{$\begin{array}{c}\text { Area } \\
\mathrm{m}^{2} \\
\text { SAR } \\
\text { Number }\end{array}$} & \multirow{2}{*}{$\begin{array}{l}\text { ATC-21 } \\
\text { Rating } \\
\text { Constur } \\
\text { Type }\end{array}$} & \multirow{2}{*}{$\begin{array}{l}\text { Priority } \\
\text { Score } \\
\text { Struct. } \\
\text { Accidents }\end{array}$} & \multirow{2}{*}{$\begin{array}{l}\text { County } \\
\text { State }\end{array}$} & \multirow{2}{*}{$\begin{array}{c}\text { Number } \\
\text { Stories } \\
\text { NPH } \\
\text { Evaluation }\end{array}$} \\
\hline & & & & & & & & & & \\
\hline $217 \mathrm{~B}$ & DEMINERALIZATI & & 1 & $10 / 54$ & & 45 & & & Benton & \\
\hline $200 \mathrm{E}$ & ON BUILDING & TRP & HEINEMAN, & MIDGETT, JOE & & & & & Washington & \\
\hline $219 \mathrm{~S}$ & RAD WASTE & & 1 & $08 / 52$ & & 27 & & & Benton & \\
\hline $200 \mathrm{~W}$ & STAGING \& & TWR & KING, A G & ERMOLD, LEON & & & & & Washington & \\
\hline $2200 \mathrm{~B}$ & CONSTRUCTION & & 1 & & & 6 & & & Benton & \\
\hline $200 E$ & PORTAL & $\mathrm{KEH}$ & FOUCAULT, I & FOUCAULT, DA & & & & & Washington & \\
\hline $2201 B$ & CONSTRUCTION & & 1 & & & 27 & & & Benton & \\
\hline $200 \mathrm{E}$ & ICE HOUSE & $\mathrm{KEH}$ & FOUCAULT, & FOUCAULT, DA & & & & & Washington & \\
\hline $221 \mathrm{~A}$ & KEH PIPEFITTER & & 1 & & & 80 & & & Benton & \\
\hline $200 \mathrm{E}$ & SHOP & $\mathrm{KEH}$ & HOLLADAY, & FOUCAULT, DA & & & & & Washington & \\
\hline $221 \mathrm{~B}$ & B PLANT & & 1 & $12 / 44$ & & 7760 & & & Benton & \\
\hline $200 \mathrm{E}$ & & $\mathrm{TRP}$ & HEINEMAN, & MIDGETT, JOE & & & & & Washington & \\
\hline $221 \mathrm{BA}$ & COOLING WATER & & I & $12 / 67$ & & 6 & & & Benton & \\
\hline $200 \mathrm{E}$ & MONITORING & TRP & HEINEMAN, & MIDGETT, JOE & & & & & Washington & \\
\hline $221 \mathrm{BB}$ & PROCESS STEAM & & 1 & $04 / 68$ & & 27 & & & Benton & \\
\hline $200 \mathrm{E}$ & AND & TRP & HEINEMAN, & MIDGETT, JOE & & & & & Washington & \\
\hline $221 \mathrm{BC}$ & SWP CHANGE & & 1 & $12 / 67$ & & 72 & & & Benton & \\
\hline $200 \mathrm{E}$ & HOUSE & TRP & HEINEMAN, F & MIDGETT, JOE & & & & & Washington & \\
\hline $221 \mathrm{BD}$ & LAUNDRY & & 1 & $06 / 65$ & & 54 & & & Benton & \\
\hline $200 E$ & STORAGE & TRP & HEINEMAN, & MIDGETT, JOE & & & & & Washington & \\
\hline
\end{tabular}


WHC-SO-SQA-CSWD-501 ReV 0

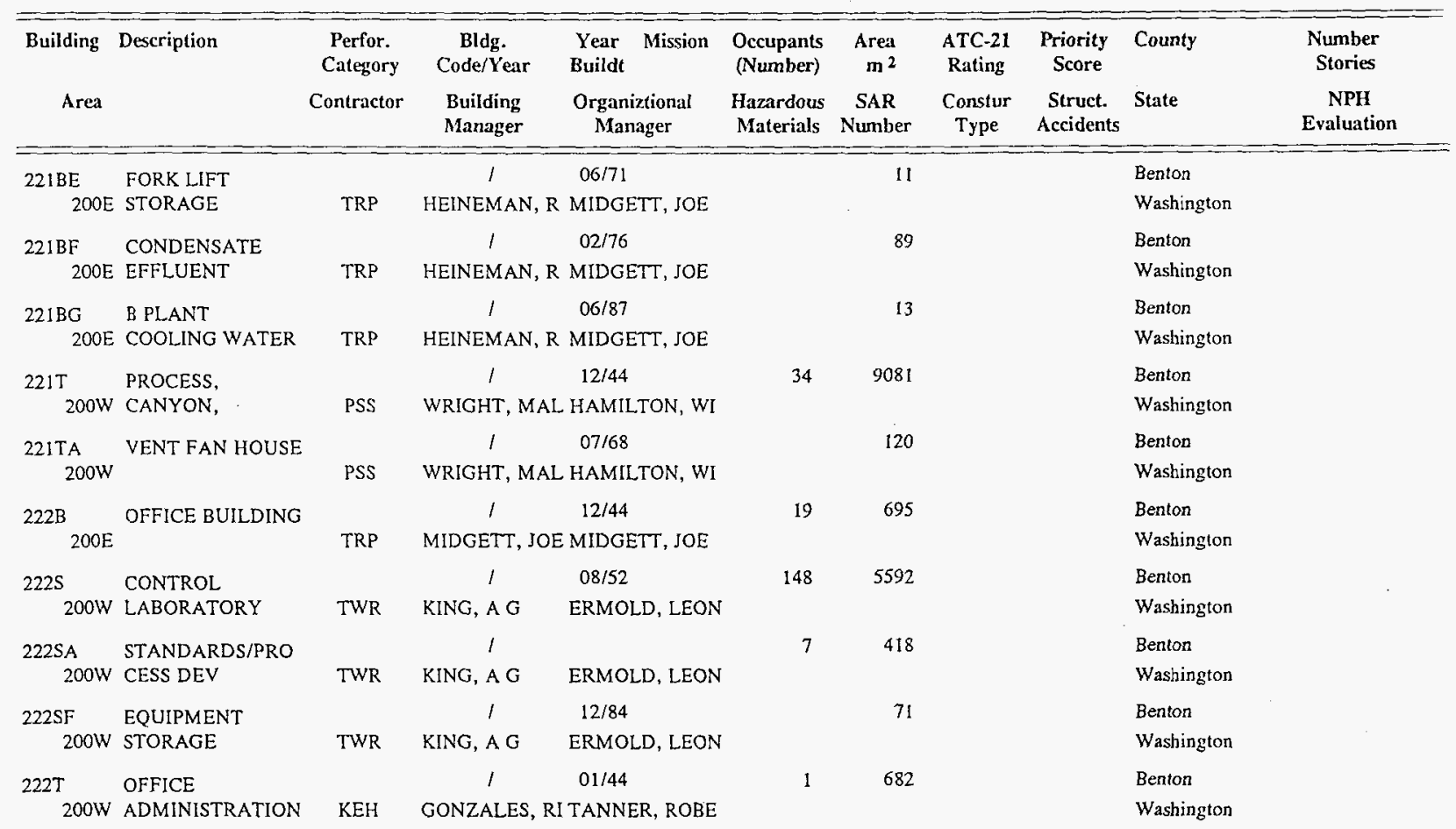


WHC-SD-SQA-CSWD-501 ReV 0

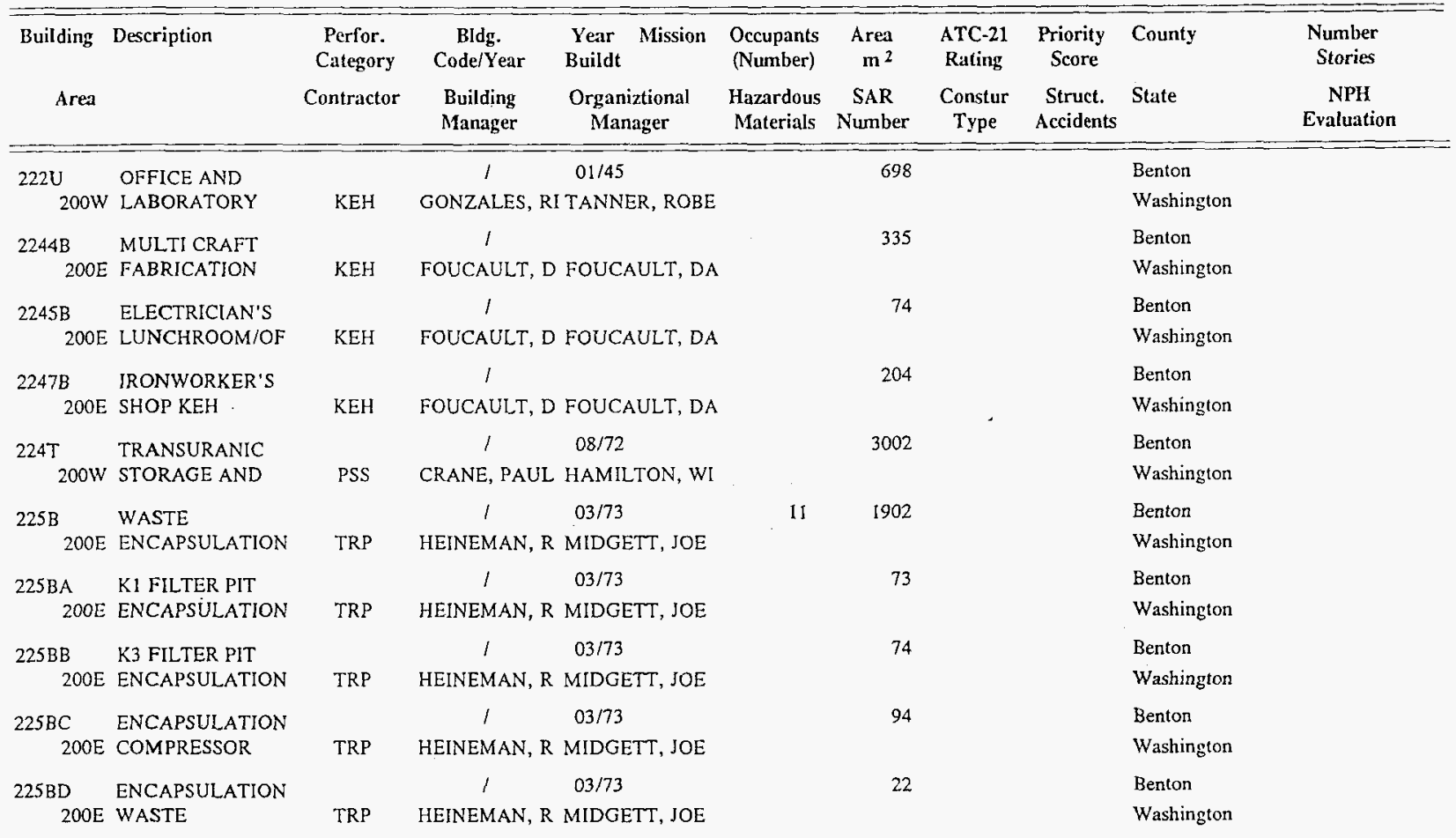


WHC-SD-SQA-CSWD-501 Rev 0

\begin{tabular}{|c|c|c|c|c|c|c|c|c|c|c|}
\hline Building & Description & $\begin{array}{c}\text { Perfor. } \\
\text { Category } \\
\text { Contractor }\end{array}$ & $\begin{array}{c}\text { Bldg. } \\
\text { Code/Year } \\
\text { Building } \\
\text { Manager }\end{array}$ & $\begin{array}{l}\text { Year Mission } \\
\text { Buildt } \\
\text { Organiztional } \\
\text { Manager }\end{array}$ & $\begin{array}{l}\text { Occupants } \\
\text { Number) } \\
\text { Hazardous } \\
\text { Materials }\end{array}$ & $\begin{array}{c}\text { Area } \\
m^{2} \\
\text { SAR } \\
\text { Number }\end{array}$ & $\begin{array}{l}\text { ATC-21 } \\
\text { Rating } \\
\text { Constur } \\
\text { Type }\end{array}$ & $\begin{array}{l}\text { Priority } \\
\text { Score } \\
\text { Struct. } \\
\text { Accidents }\end{array}$ & State & $\begin{array}{c}\text { Number } \\
\text { Stories } \\
\text { NPH } \\
\text { Evaluation }\end{array}$ \\
\hline $225 \mathrm{BE}$ & ENCAPSULATION & & 1 & $09 / 77$ & 13 & 297 & & & Benton & \\
\hline $200 E$ & MAINTENANCE & TRP & GONZALES, RI & I MIDGETT, JOE & & & & & Washington & \\
\hline $225 \mathrm{E}$ & PUMP STATION & & 1 & $12 / 94$ & & 84 & & & Benton. & \\
\hline $200 \mathrm{E}$ & NO.2 \& LOCAL & PSS & WURZ, RICHA & DILIBERTO, AN & & & & & Washington & \\
\hline $225 \mathrm{EC}$ & LOCAL CONTROL & & 1 & $12 / 94$ & & 11 & & & Benton & \\
\hline $200 E$ & UNIT $55 \mathrm{C}-13$ & TRP & WURZ, RICHA & HAMRICK, DOU & & & & & Washington & \\
\hline $225 \mathrm{~W}$ & PUMP STATION & & 1 & $12 / 94$ & & 84 & & & Benton & \\
\hline $200 \mathrm{~W}$ & NO.I \& LOCAL & PSS & WURZ, RICHA & DILIBERTO, AN & & & & & Washington & \\
\hline 225WA & LOCAL CNTRL & & 1 & $12 / 94$ & & 11 & & & Benton & \\
\hline $200 \mathrm{~W}$ & UNIT $55 \mathrm{C}-21$ & PSS & HAMILTON, W & HAMILTON, WI & & & & & Washington & \\
\hline $225 \mathrm{WB}$ & LOCAL CNTRL & & 1 & $12 / 94$ & & 11 & & & Benton & \\
\hline $200 \mathrm{~W}$ & UNIT 55C-22 & TWR & $\mathrm{KING}, \mathrm{A} \mathrm{G}$ & ERMOLD, LEON & & & & & Washington & \\
\hline $225 \mathrm{WC}$ & INSTRUMENTATI & & 1 & $12 / 94$ & & 28 & & & Benton & \\
\hline $200 \mathrm{~W}$ & ON \& LOCAL & TRP & BURKS, DAVI & VOGT, ERIC C & & & $\cdot$ & & Washington & \\
\hline 226 ISTV & 2261 STEVENS & & 1 & & & & & & Benton & \\
\hline $\mathrm{RCHN}$ & DRIVE & $\mathrm{KEH}$ & TANNER RM & & & & & & Washington & \\
\hline $2300 \mathrm{~W}$ & ELECTRICIAN'S & & 1 & & & 149 & & & Benton & \\
\hline $200 W$ & SHOP & $\mathrm{KEH}$ & HOLLADAY, J & FOUCAULT, DA & & & & & Washington & \\
\hline $2304 W$ & PIPEFITTER'S & & 1 & & & 112 & & & Benton & \\
\hline $200 \mathrm{~W}$ & SHOP & $\mathrm{KEH}$ & HOLLADAY, J & FOUCAULT, DA & & & & & Washington & \\
\hline
\end{tabular}


WHC-SD-SQA-CSWD-501 ReV 0

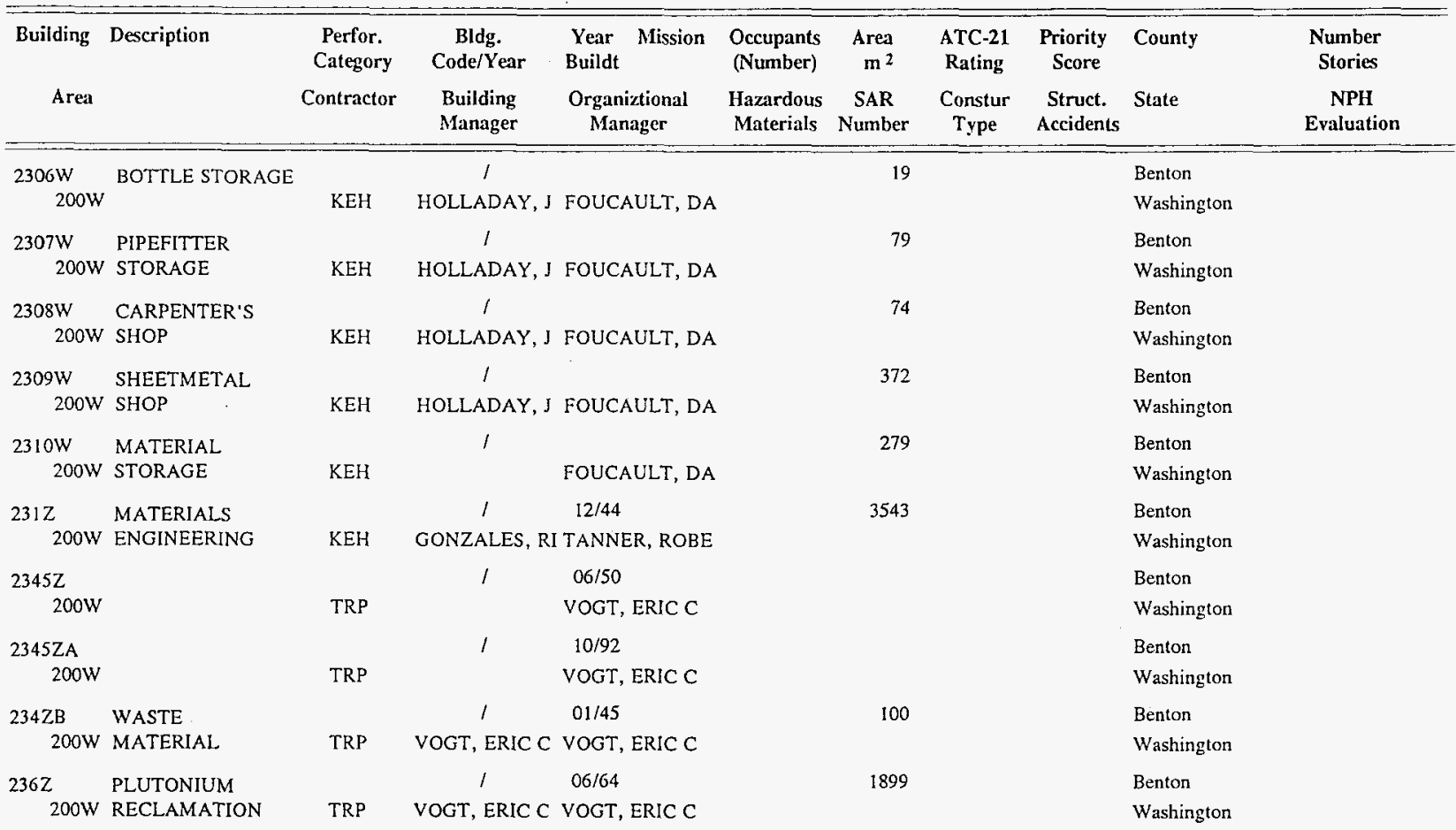


WHC-SD-SQA-CSWD-501 Rev 0

\begin{tabular}{|c|c|c|c|c|c|c|c|c|c|c|}
\hline Building & Description & $\begin{array}{c}\text { Perfor. } \\
\text { Category } \\
\text { Contractor }\end{array}$ & $\begin{array}{c}\text { Bldg. } \\
\text { Code/Year } \\
\text { Building } \\
\text { Manager }\end{array}$ & $\begin{array}{l}\text { Year Mission } \\
\text { Buildt } \\
\text { Organiztional } \\
\text { Manager }\end{array}$ & $\begin{array}{l}\text { Occupants } \\
\text { (Number) } \\
\text { Hazardous } \\
\text { Materials }\end{array}$ & $\begin{array}{c}\text { Area } \\
\mathrm{m}^{2} \\
\text { SAR } \\
\text { Number }\end{array}$ & $\begin{array}{l}\text { ATC-21 } \\
\text { Rating } \\
\text { Constur } \\
\text { Type }\end{array}$ & $\begin{array}{l}\text { Priority } \\
\text { Score } \\
\text { Struct. } \\
\text { Accidents }\end{array}$ & State & $\begin{array}{c}\text { Number } \\
\text { Storjes } \\
\text { NPH } \\
\text { Evaluation }\end{array}$ \\
\hline $2400 E$ & DRY MATERIAL & & 1 & $04 / 87$ & & 46 & & & Benton & \\
\hline $200 \mathrm{E}$ & FACILITY & TWR & BADDEN, JAM & WICKS, JAMES & & & & & Washington & \\
\hline $2401 \mathrm{~W}$ & MIXED WASTE & & 1 & $04 / 89$ & & 372 & & & Benton & \\
\hline $200 \mathrm{~W}$ & STORAGE & PSS & CRANE, PAUL & HAMILTON, WI & & & & & Washington & \\
\hline $2402 W$ & MIXED WASTE & & / & $02 / 91$ & & 372 & & & Benton & \\
\hline $200 \mathrm{~W}$ & STORAGE & PSS & CRANE, PAUL & HAMILTON, WI & & & & & Washington & \\
\hline $2402 W B$ & MIXED WASTE & & 1 & $11 / 90$ & & 372 & & & Benton & \\
\hline $200 w$ & STORAGE & PSS & CRANE, PAUL & HAMILTON, WI & & & & & Washington & \\
\hline $2402 \mathrm{WC}$ & MIXED WASTE & & 1 & $11 / 90$ & & 372 & & & Benton & \\
\hline $200 \mathrm{~W}$ & STORAGE & PSS & CRANE, PAUL & HAMILTON, WI & & & & & Washington & \\
\hline $2402 W D$ & MIXED WASTE & & 1 & $11 / 90$ & & 372 & & & Benton & \\
\hline $200 \mathrm{~W}$ & STORAGE & PSS & CRANE, PAUL & HAMILTON, WI & & & & & Washington & \\
\hline 2402WE & MIXED WASTE & & 1 & $11 / 90$ & & 372 & & & Benton & \\
\hline $200 \mathrm{~W}$ & STORAGE & PSS & CRANE, PAUL & HAMILTON, WI & & & & & Washington & \\
\hline $2402 W F$ & MIXED WASTE & & 1 & $11 / 90$ & & 372 & & & Benton & \\
\hline $200 \mathrm{~W}$ & STORAGE & PSS & CRANE, PAUL & HAMILTON, WI & & & & & Washington & \\
\hline $2402 W G$ & RMW STORAGE & & I & $11 / 90$ & & 372 & & & Benton & \\
\hline $200 \mathrm{~W}$ & FACILITY \#7 & PSS & CRANE, PAUL & HAMILTON, WI & & & & & Washington & \\
\hline $2402 \mathrm{WH}$ & RMW STORAGE & & 1 & $11 / 90$ & & 372 & & & Benton & \\
\hline $200 \mathrm{~W}$ & FACILITY $\# 8$ & PSS & CRANE, PAUL & HAMILTON, WI & & & & & Washington & \\
\hline
\end{tabular}


WHC-SD-SQA-CSWD-501 Rev 0

\begin{tabular}{|c|c|c|c|c|c|c|c|c|c|c|}
\hline Area & Description & $\begin{array}{c}\text { Perfor. } \\
\text { Category } \\
\text { Contractor }\end{array}$ & $\begin{array}{l}\text { Bldg. } \\
\text { Code/Year } \\
\text { Building } \\
\text { Manager }\end{array}$ & $\begin{array}{l}\text { Year Mission } \\
\text { Buildt } \\
\text { Organiztional } \\
\text { Manager }\end{array}$ & $\begin{array}{c}\text { Occupants } \\
\text { (Number) } \\
\text { Hazardous } \\
\text { Materials }\end{array}$ & $\begin{array}{c}\text { Area } \\
\text { m }^{2} \\
\text { SAR } \\
\text { Number }\end{array}$ & $\begin{array}{c}\text { ATC-21 } \\
\text { Rating } \\
\text { Constur } \\
\text { Type }\end{array}$ & $\begin{array}{l}\text { Priority } \\
\text { Score } \\
\text { Struct. } \\
\text { Accidents }\end{array}$ & State & $\begin{array}{c}\text { Number } \\
\text { Stories } \\
\text { NPH } \\
\text { Evaluation }\end{array}$ \\
\hline $\begin{array}{l}2402 \mathrm{WI} \\
200 \mathrm{~W}\end{array}$ & $\begin{array}{l}\text { RMW STORAGE } \\
\text { FACILITY }\end{array}$ & PSS & $\begin{array}{c}\text { ' } \\
\text { CRANE, PAUL }\end{array}$ & $\begin{array}{c}11 / 90 \\
\text { HAMILTON, WI }\end{array}$ & & 372 & & & $\begin{array}{l}\text { Benton } \\
\text { Washington }\end{array}$ & \\
\hline $\begin{array}{l}2402 \mathrm{WJ} \\
200 \mathrm{~W}\end{array}$ & $\begin{array}{l}\text { RMW STORAGE } \\
\text { FACILITY } \# 10\end{array}$ & PSS & $\begin{array}{c}/ \\
\text { CRANE, PAUL }\end{array}$ & $\begin{array}{l}11 / 90 \\
\text { HAMILTON, WI }\end{array}$ & & 372 & & & $\begin{array}{l}\text { Benton } \\
\text { Washington }\end{array}$ & \\
\hline $\begin{array}{l}2402 \mathrm{WK} \\
200 \mathrm{~W}\end{array}$ & $\begin{array}{l}\text { RMW STORAGE } \\
\text { FACILITY \#I } 1\end{array}$ & PSS & $\begin{array}{c}/ \\
\text { CRANE, PAUL }\end{array}$ & $\begin{array}{l}11 / 90 \\
\text { HAMILTON, WI }\end{array}$ & & 372 & & & $\begin{array}{l}\text { Benton } \\
\text { Washington }\end{array}$ & \\
\hline $\begin{array}{l}2402 \mathrm{WL} \\
200 \mathrm{~W}\end{array}$ & $\begin{array}{l}\text { RMW STORAGE } \\
\text { FACILITY } \# 12\end{array}$ & PSS & $\begin{array}{c}\text { / } \\
\text { CRANE, PAUL }\end{array}$ & $\begin{array}{l}11 / 90 \\
\text { HAMILTON, WI }\end{array}$ & & 372 & & & $\begin{array}{l}\text { Benton } \\
\text { Washington }\end{array}$ & \\
\hline $\begin{array}{l}2403 \mathrm{E} \\
200 \mathrm{E}\end{array}$ & $\begin{array}{l}\text { DMRHF DRY } \\
\text { BLENDED }\end{array}$ & TWR & $\begin{array}{c}/ \\
\text { BADDEN, JAM }\end{array}$ & $\begin{array}{l}04 / 87 \\
1 \text { WICKS, JAMES }\end{array}$ & & 19 & & & $\begin{array}{l}\text { Benton } \\
\text { Washington }\end{array}$ & \\
\hline $\begin{array}{l}2403 \mathrm{EA} \\
200 \mathrm{E}\end{array}$ & $\begin{array}{l}\text { COMPRESSOR } \\
\text { LEANTO }\end{array}$ & TWR & $\begin{array}{c}\text { I } \\
\text { MURKOWSKI, }\end{array}$ & $\begin{array}{l}04 / 87 \\
\text { WICKS, JAMES }\end{array}$ & & 24 & & & $\begin{array}{l}\text { Benton } \\
\text { Washington }\end{array}$ & \\
\hline $\begin{array}{l}2403 \mathrm{WA} \\
200 \mathrm{~W}\end{array}$ & $\begin{array}{l}\text { RMW STORAGE } \\
\text { FACILITY }\end{array}$ & PSS & $\begin{array}{c}\text { ' } \\
\text { CRANE, PAUL }\end{array}$ & $\begin{array}{l}10 / 92 \\
\text { HAMILTON, WI }\end{array}$ & & 3160 & & & $\begin{array}{l}\text { Benton } \\
\text { Washington }\end{array}$ & \\
\hline $\begin{array}{l}2403 \mathrm{WB} \\
200 \mathrm{~W}\end{array}$ & $\begin{array}{l}\text { RMW STORAGE } \\
\text { FACILITY }\end{array}$ & PSS & $\begin{array}{c}\text { I } \\
\text { CRANE, PAUL }\end{array}$ & $\begin{array}{l}10 / 92 \\
\text { HAMILTON, WI }\end{array}$ & & 3160 & & & $\begin{array}{l}\text { Benton } \\
\text { Washington }\end{array}$ & \\
\hline $\begin{array}{l}2403 \mathrm{WC} \\
200 \mathrm{~W}\end{array}$ & $\begin{array}{l}\text { RMW STORAGE } \\
\text { FACILITY }\end{array}$ & PSS & $\begin{array}{c}\text { I } \\
\text { CRANE, PAUL }\end{array}$ & $\begin{array}{l}10 / 92 \\
\text { HAMILTON, WI }\end{array}$ & & 3160 & & & $\begin{array}{l}\text { Benton } \\
\text { Washington }\end{array}$ & \\
\hline $\begin{array}{l}2403 \mathrm{WD} \\
200 \mathrm{~W}\end{array}$ & $\begin{array}{l}\text { RMW STORAGE } \\
\text { FACILITY }\end{array}$ & PSS & $\begin{array}{c}\prime \\
\text { CRANE, PAUL }\end{array}$ & $\begin{array}{l}10 / 92 \\
\text { HAMILTON, WI }\end{array}$ & & 5135 & & & $\begin{array}{l}\text { Benton } \\
\text { Washington }\end{array}$ & \\
\hline
\end{tabular}


WHC-SO-SQA-CSWO-501 ReV 0

\begin{tabular}{|c|c|c|c|c|c|c|c|c|c|c|}
\hline Building & Description & $\begin{array}{c}\text { Perfor. } \\
\text { Category } \\
\text { Contractor }\end{array}$ & $\begin{array}{c}\text { Bldg. } \\
\text { Code/Year } \\
\text { Building } \\
\text { Manager }\end{array}$ & $\begin{array}{l}\text { Year Mission } \\
\text { Buildt } \\
\text { Organiztional } \\
\quad \text { Manager }\end{array}$ & $\begin{array}{c}\text { Occupants } \\
\text { (Number) } \\
\text { Hazardous } \\
\text { Materials }\end{array}$ & $\begin{array}{c}\text { Area } \\
\mathrm{m}^{2} \\
\text { SAR } \\
\text { Number }\end{array}$ & $\begin{array}{c}\text { ATC-21 } \\
\text { Rating } \\
\text { Constur } \\
\text { Type }\end{array}$ & $\begin{array}{l}\text { Priority } \\
\text { Score } \\
\text { Struct. } \\
\text { Accidents }\end{array}$ & State & $\begin{array}{c}\text { Number } \\
\text { Stories } \\
\text { NPH } \\
\text { Evaluation }\end{array}$ \\
\hline $\begin{array}{l}2404 \mathrm{E} \\
200 \mathrm{E}\end{array}$ & $\begin{array}{l}\text { DMRHF } \\
\text { COMPRESSOR }\end{array}$ & TWR & $\begin{array}{c}/ \\
\text { BADDEN, JAM }\end{array}$ & $\begin{array}{l}04 / 87 \\
\text { WICKS, JAMES }\end{array}$ & & & & & $\begin{array}{l}\text { Benton } \\
\text { Washington }\end{array}$ & \\
\hline $\begin{array}{l}2420 \text { STV } \\
\text { RCHN }\end{array}$ & $\begin{array}{l}2420 \text { STEVENS } \\
\text { CTR CIR }\end{array}$ & $\mathrm{KEH}$ & $\begin{array}{c}/ \\
\text { TANNER RM }\end{array}$ & & 289 & & & & $\begin{array}{l}\text { Benton } \\
\text { Washington }\end{array}$ & \\
\hline $\begin{array}{l}\text { 2425STV } \\
\text { RCHN }\end{array}$ & $\begin{array}{l}2425 \text { STEVENS } \\
\text { CTR CIR }\end{array}$ & $\mathrm{KEH}$ & $\begin{array}{c}\prime \\
\text { TANNER RM }\end{array}$ & & 107 & & & & $\begin{array}{l}\text { Benton } \\
\text { Washington }\end{array}$ & \\
\hline $\begin{array}{l}242 A \\
200 E\end{array}$ & EVAPORATOR & TWR & $\begin{array}{c}\text { / } \\
\text { GEARY, JAME }\end{array}$ & $\begin{array}{l}04 / 77 \\
\text { WICKS, JAMES }\end{array}$ & 21 & 777 & & & $\begin{array}{l}\text { Benton } \\
\text { Washington }\end{array}$ & \\
\hline $\begin{array}{l}242 \mathrm{~A} 81 \\
200 \mathrm{E}\end{array}$ & $\begin{array}{l}\text { WATER SERVICE } \\
\text { BUILDING }\end{array}$ & TWR & 1 & WICKS, JAMES & & 52 & & & $\begin{array}{l}\text { Benton } \\
\text { Washington }\end{array}$ & \\
\hline $\begin{array}{l}242 \mathrm{AC} \\
200 \mathrm{E}\end{array}$ & $\begin{array}{l}\text { PIPEFITTERS } \\
\text { SHOP }\end{array}$ & $\mathrm{KEH}$ & $\begin{array}{c}/ \\
\text { DEMPSEY, KEI }\end{array}$ & I DEMPSEY, KEIT & & 74 & & & $\begin{array}{l}\text { Benton } \\
\text { Washington }\end{array}$ & \\
\hline $\begin{array}{l}\text { 242AL11 } \\
200 \mathrm{E}\end{array}$ & $\begin{array}{l}\text { LERF STORAGE } \\
\text { BUILDING }\end{array}$ & TWR & $\begin{array}{c}\prime \\
\text { GEARY, JAME }\end{array}$ & $\begin{array}{l}07 / 94 \\
\text { WICKS, JAMES }\end{array}$ & & 46 & & & $\begin{array}{l}\text { Benton } \\
\text { Washington }\end{array}$ & \\
\hline $\begin{array}{l}242 S \\
200 W\end{array}$ & $\begin{array}{l}\text { EVAPORATOR } \\
\text { FACILITY }\end{array}$ & TWR & $\begin{array}{c}l \\
\text { WICKS, JAMES }\end{array}$ & $\begin{array}{l}03 / 74 \\
\text { WICKS, JAMES }\end{array}$ & & & & & $\begin{array}{l}\text { Benton } \\
\text { Washington }\end{array}$ & \\
\hline $\begin{array}{l}242 \mathrm{~T} \\
200 \mathrm{~W}\end{array}$ & $\begin{array}{l}\text { WASTE } \\
\text { DISPOSAL }\end{array}$ & TWR & $\begin{array}{c}\prime \\
\text { WICKS, JAMES }\end{array}$ & $\begin{array}{l}\text { 05/51 } \\
\text { WICKS, JAMES }\end{array}$ & & 252 & & & $\begin{array}{l}\text { Benton } \\
\text { Washington }\end{array}$ & \\
\hline $\begin{array}{l}242 \mathrm{~TB} \\
200 \mathrm{~W}\end{array}$ & VENT HOUSE & TWR & $\begin{array}{c}1 \\
\text { ROSS, WILLIA }\end{array}$ & $\begin{array}{l}11 / 73 \\
\text { WICKS, JAMES }\end{array}$ & & 18 & & & $\begin{array}{l}\text { Benton } \\
\text { Washington }\end{array}$ & \\
\hline
\end{tabular}


WHC-SD-SQA-CSWD-501 ReV 0

\begin{tabular}{|c|c|c|c|c|c|c|c|c|c|c|}
\hline Building & Description & $\begin{array}{l}\text { Perfor. } \\
\text { Category } \\
\text { Contractor }\end{array}$ & $\begin{array}{c}\text { Bldg. } \\
\text { Code/Year } \\
\text { Building } \\
\text { Manager }\end{array}$ & $\begin{array}{l}\text { Year Mission } \\
\text { Buildt } \\
\text { Organiztional } \\
\text { Manager }\end{array}$ & $\begin{array}{c}\text { Occupants } \\
\text { (Number) } \\
\text { Hazardous } \\
\text { Materials }\end{array}$ & $\begin{array}{c}\text { Area } \\
\mathbf{m}^{2} \\
\text { SAR } \\
\text { Number }\end{array}$ & $\begin{array}{c}\text { ATC-21 } \\
\text { Rating } \\
\text { Constur } \\
\text { Type }\end{array}$ & $\begin{array}{l}\text { Priority } \\
\text { Score } \\
\text { Struct. } \\
\text { Accidents }\end{array}$ & State & $\begin{array}{c}\text { Number } \\
\text { Stories } \\
\text { NPH } \\
\text { Evaluation }\end{array}$ \\
\hline $\begin{array}{l}242 \mathrm{TC} \\
200 \mathrm{~W}\end{array}$ & $\begin{array}{l}\text { TANK FARM } \\
\text { MICROCOMPUTE }\end{array}$ & TWR & $\begin{array}{c}\text { / } \\
\text { WICKS, JAMES }\end{array}$ & $\begin{array}{l}07 / 80 \\
\text { WICKS, JAMES }\end{array}$ & & & & & $\begin{array}{l}\text { Benton } \\
\text { Washington }\end{array}$ & \\
\hline $\begin{array}{l}242 Z \\
200 w\end{array}$ & $\begin{array}{l}\text { WASTE } \\
\text { TREATMENT }\end{array}$ & TRP & $\begin{array}{c}\text { / } \\
\text { VOGT, ERIC C }\end{array}$ & $\begin{array}{l}\text { 06/64 } \\
\text { VOGT, ERIC C }\end{array}$ & & 98 & & & $\begin{array}{l}\text { Benton } \\
\text { Washington }\end{array}$ & \\
\hline $\begin{array}{l}242 \mathrm{ZA} \\
200 \mathrm{~W}\end{array}$ & $\begin{array}{l}\text { MONITORING } \\
\text { BUILDING }\end{array}$ & TRP & VOGT, ERIC C & $\begin{array}{l}\text { O5/87 } \\
\text { VOGT, ERIC C }\end{array}$ & & & & & $\begin{array}{l}\text { Benton } \\
\text { Washington }\end{array}$ & \\
\hline $\begin{array}{l}\text { 2430STV } \\
\text { RCHN }\end{array}$ & $\begin{array}{l}2430 \text { STEVENS } \\
\text { CTR CIR }\end{array}$ & KEH & $\begin{array}{c}/ \\
\text { TANNER RM }\end{array}$ & & 133 & & & & $\begin{array}{l}\text { Benton } \\
\text { Washington }\end{array}$ & \\
\hline $\begin{array}{l}243 G \\
200 E\end{array}$ & GROUT FACILITY & TWR & $\begin{array}{c}l \\
\text { RAVENCRAFT }\end{array}$ & $\begin{array}{l}08 / 86 \\
\text { WICKS, JAMES }\end{array}$ & & & & & $\begin{array}{l}\text { Benton } \\
\text { Washington }\end{array}$ & \\
\hline $\begin{array}{l}243 \mathrm{Gl} \\
200 \mathrm{E}\end{array}$ & $\begin{array}{l}\text { GROUT } \\
\text { PROCESSING }\end{array}$ & TWR & 1 & & & & & & $\begin{array}{l}\text { Benton } \\
\text { Washington }\end{array}$ & \\
\hline $\begin{array}{l}243 \mathrm{G} 1 \mathrm{~A} \\
200 \mathrm{E}\end{array}$ & $\begin{array}{l}\text { GROUT } \\
\text { PROCESSING }\end{array}$ & TWR & $\begin{array}{c}\text { I } \\
\text { RAVENCRAFT }\end{array}$ & & & 33 & & & $\begin{array}{l}\text { Benton } \\
\text { Washington }\end{array}$ & \\
\hline $\begin{array}{l}243 \mathrm{G} 4 \\
200 \mathrm{E}\end{array}$ & $\begin{array}{l}\text { GPF CONTROL } \\
\text { ROOM MODULE }\end{array}$ & PSS & $\begin{array}{c}/ \\
\text { RAVENCRAFT }\end{array}$ & & 1 & 19 & & & $\begin{array}{l}\text { Benton } \\
\text { Washington }\end{array}$ & \\
\hline $\begin{array}{l}243 G 5 \\
200 E\end{array}$ & $\begin{array}{l}\text { GPF STANDBY } \\
\text { GENERATOR }\end{array}$ & TWR & 1 & & & & & & $\begin{array}{l}\text { Benton } \\
\text { Washington }\end{array}$ & \\
\hline $\begin{array}{l}243 \mathrm{G} 8 \\
200 \mathrm{E}\end{array}$ & $\begin{array}{l}\text { GPF FILTRATION } \\
\text { MODULE }\end{array}$ & $\mathrm{KEH}$ & 1 & & & & & & $\begin{array}{l}\text { Benton } \\
\text { Washington }\end{array}$ & \\
\hline
\end{tabular}


WHC-SD-SQA-CSWD-501 Rev 0

\begin{tabular}{|c|c|c|c|c|c|c|c|c|c|c|}
\hline Building & Description & $\begin{array}{l}\text { Perfor. } \\
\text { Category }\end{array}$ & $\begin{array}{c}\text { Bldg. } \\
\text { Code/Year }\end{array}$ & $\begin{array}{l}\text { Year Mission } \\
\text { Buildt }\end{array}$ & $\begin{array}{c}\text { Occupants } \\
\text { (Number) }\end{array}$ & $\begin{array}{r}\text { Area } \\
\mathrm{m}^{2}\end{array}$ & $\begin{array}{l}\text { ATC-21 } \\
\text { Rating }\end{array}$ & $\begin{array}{c}\text { Priority } \\
\text { Score }\end{array}$ & County & $\begin{array}{l}\text { Number } \\
\text { Stories }\end{array}$ \\
\hline Area & & Contractor & $\begin{array}{l}\text { Building } \\
\text { Manager }\end{array}$ & $\begin{array}{l}\text { Organiztional } \\
\text { Manager }\end{array}$ & $\begin{array}{c}\text { Hazardous } \\
\text { Materials }\end{array}$ & $\begin{array}{c}\text { SAR } \\
\text { Number }\end{array}$ & $\begin{array}{l}\text { Constur } \\
\text { Type }\end{array}$ & $\begin{array}{l}\text { Struct. } \\
\text { Accidents }\end{array}$ & State & $\begin{array}{c}\text { NPH } \\
\text { Evaluation }\end{array}$ \\
\hline $243 G 82$ & GROUT & & 1 & & & 5 & & & Benton & \\
\hline $200 E$ & PROCESSING & TWR & & & & & & & Washington & \\
\hline $243 \mathrm{TGE}$ & & & 1 & & & & & & Benton & \\
\hline $200 E$ & & TWR & & & & & & & Washington & \\
\hline $243 Z$ & LOW LEVEL & & 1 & $12 / 93$ & & 228 & & & Benton & \\
\hline $200 \mathrm{~W}$ & WASTE & TRP & TALBOT, MIC & VOGT, ERIC C & & & & & Washington & \\
\hline $2440 \mathrm{STV}$ & 2440 STEVENS & & l & & 346 & & & & Benton & \\
\hline $\mathrm{RCHN}$ & CTR CIR & $\mathrm{KEH}$ & TANNER RM & & & & & & Washington & \\
\hline $244 \mathrm{~A}$ & WASTE VAULT & & 1 & $04 / 77$ & & 11 & & & Benton & \\
\hline $200 \mathrm{E}$ & AND & TWR & RAVENCRAFT & WICKS, JAMES & & & & & Washington & \\
\hline $244 \mathrm{AR}$ & SLUDGE VAULT & & 1 & $01 / 68$ & & 347 & & & Benton & \\
\hline $200 E$ & STORAGE AND & TWR & RAVENCRAFT & WICKS, JAMES & & & & & Washington & \\
\hline 244AR701 & EMERGENCY & & 1 & $06 / 73$ & & 7 & & & Benton & \\
\hline $200 E$ & GENERATOR & TWR & RAVENCRAFT & - WICKS, JAMES & & & & & Washington & \\
\hline 244AR715 & COMPRESSOR & & 1 & $12 / 87$ & & & & & Benton & \\
\hline $200 \mathrm{E}$ & BUILDING & TWR & RAVENCRAFT & WICKS, JAMES & & & & & Washington & \\
\hline $244 B X$ & SALT WELL & & 1 & & & 13 & & & Benton & \\
\hline $200 \mathrm{E}$ & RECEIVER VAULT & TWR & REBER, DAVI & WICKS, JAMES & & & & & Washington & \\
\hline $244 S 271$ & INSTRUMENT & & 1 & $04 / 81$ & & 18 & & & Benton & \\
\hline $200 \mathrm{~W}$ & CONTROL HOUSE & TWR & WICKS, JAMES & WICKS, JAMES & & & & & Washington & \\
\hline
\end{tabular}


WHC-SD-SQA-CSWD-501 Rev 0

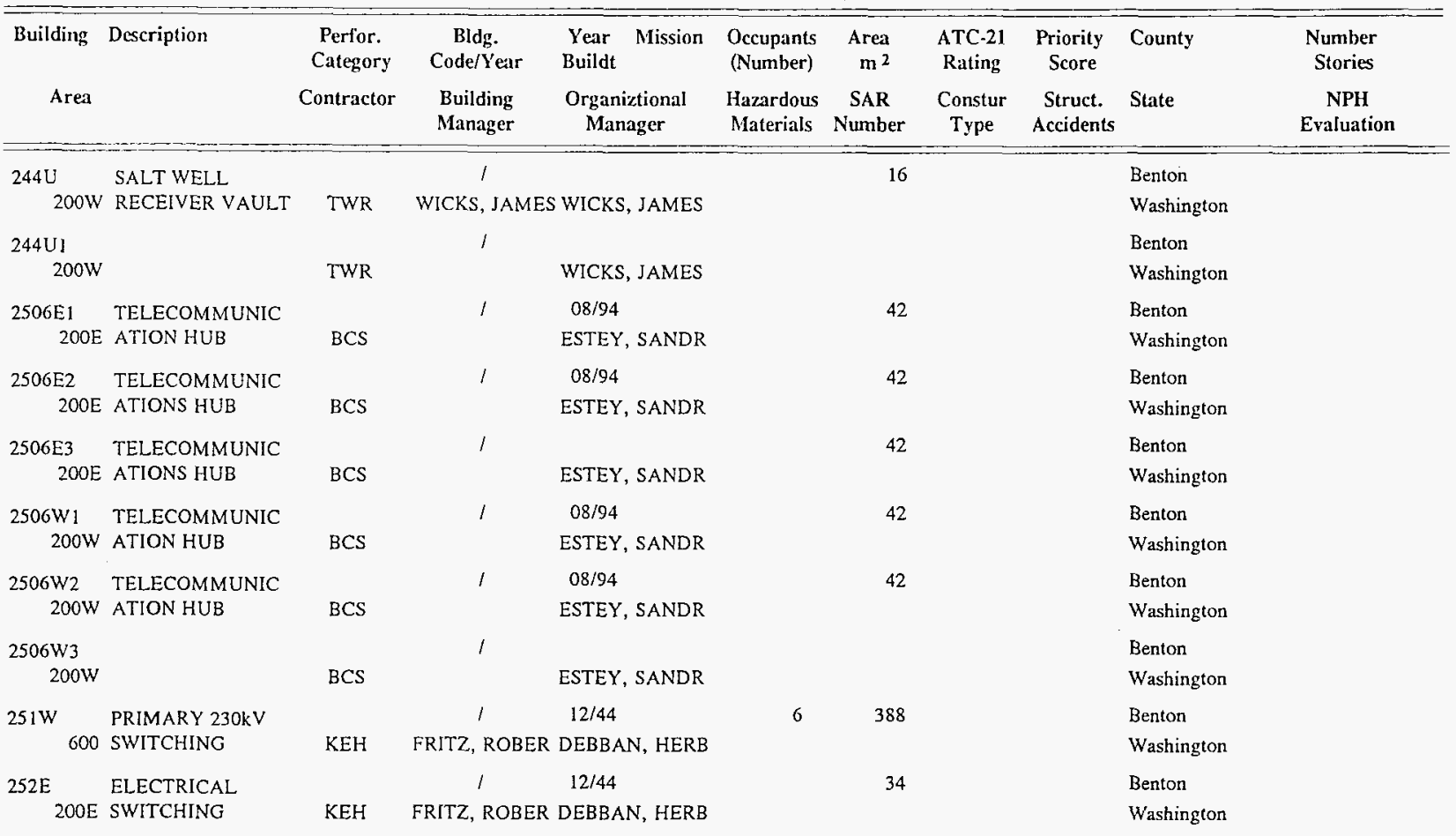


WHC-SD-SQA-CSWD-501 Rev 0

\begin{tabular}{|c|c|c|c|c|c|c|c|c|c|c|}
\hline Building & Description & $\begin{array}{l}\text { Perfor. } \\
\text { Category }\end{array}$ & $\begin{array}{c}\text { Bldg. } \\
\text { Code/Year }\end{array}$ & $\begin{array}{l}\text { Year Mission } \\
\text { Buildt }\end{array}$ & $\begin{array}{l}\text { Occupants } \\
\text { (Number) }\end{array}$ & $\begin{array}{r}\text { Area } \\
\mathbf{m}^{2}\end{array}$ & $\begin{array}{c}\text { ATC-21 } \\
\text { Ruting }\end{array}$ & $\begin{array}{l}\text { Priority } \\
\text { Score }\end{array}$ & County & $\begin{array}{l}\text { Number } \\
\text { Stories }\end{array}$ \\
\hline Area & & Contractor & $\begin{array}{l}\text { Building } \\
\text { Manager }\end{array}$ & $\begin{array}{l}\text { Organiztional } \\
\text { Manager }\end{array}$ & $\begin{array}{c}\text { Hazardous } \\
\text { Materials }\end{array}$ & $\begin{array}{c}\text { SAR } \\
\text { Number }\end{array}$ & $\begin{array}{l}\text { Constur } \\
\text { Type }\end{array}$ & $\begin{array}{c}\text { Struct. } \\
\text { Accidents }\end{array}$ & State & $\begin{array}{c}\text { NPH } \\
\text { Evaluation }\end{array}$ \\
\hline $\begin{array}{l}252 \mathrm{~S} \\
200 \mathrm{~W}\end{array}$ & $\begin{array}{l}\text { SUBSTATION } \\
\text { BUILDING } 13.8 \mathrm{KV}\end{array}$ & $\mathrm{KEH}$ & 1 & $\begin{array}{l}06 / 96 \\
\text { DEBBAN, HERB }\end{array}$ & & 75 & & & $\begin{array}{l}\text { Benton } \\
\text { Washington }\end{array}$ & \\
\hline $\begin{array}{r}252 \mathrm{~W} \\
200 \mathrm{~W}\end{array}$ & $\begin{array}{l}\text { ELECTRICAL } \\
\text { SWITCHING }\end{array}$ & $\mathrm{KEH}$ & $\begin{array}{c}\text { / } \\
\text { FRITZ, ROBER }\end{array}$ & $\begin{array}{c}12 / 44 \\
\text { DEBBAN, HERB }\end{array}$ & & 34 & & & $\begin{array}{l}\text { Benton } \\
\text { Washington }\end{array}$ & \\
\hline $\begin{array}{l}254 \mathrm{BY} \\
200 \mathrm{E}\end{array}$ & CONTROL HOUSE & TWR & $\begin{array}{c}/ \\
\text { SPARKS, DAVI }\end{array}$ & $\begin{array}{l}02 / 68 \\
\text { WICKS, JAMES }\end{array}$ & & 69 & & & $\begin{array}{l}\text { Benton - } \\
\text { Washington }\end{array}$ & \\
\hline $\begin{array}{l}2614 \mathrm{E} 2 \\
200 \mathrm{E}\end{array}$ & $\begin{array}{l}\text { ENVIRONMENTA } \\
\text { L MONITORING }\end{array}$ & WAE & 1 & DIXON, WILLIA & & 5 & & & $\begin{array}{l}\text { Benton } \\
\text { Washington }\end{array}$ & \\
\hline $\begin{array}{l}2614 \mathrm{~W} 2 \\
200 \mathrm{~W}\end{array}$ & $\begin{array}{l}\text { ENVIRONMENTA } \\
\text { L MONITORING }\end{array}$ & WAE & $\begin{array}{c}\prime \\
\text { DIXON, WILLI }\end{array}$ & DIXON, WILLIA & & 5 & & & $\begin{array}{l}\text { Benton } \\
\text { Washington }\end{array}$ & \\
\hline $\begin{array}{l}2614 \mathrm{~W} 4 \\
200 \mathrm{~W}\end{array}$ & $\begin{array}{l}\text { MONITOR } \\
\text { STATION }\end{array}$ & WAE & $\begin{array}{c}/ \\
\text { DIXON, WILLI }\end{array}$ & DIXON, WILLIA & & 5 & & & $\begin{array}{l}\text { Benton } \\
\text { Washington }\end{array}$ & \\
\hline $\begin{array}{l}2614 \mathrm{~W} 5 \\
200 \mathrm{~W}\end{array}$ & $\begin{array}{l}\text { ENVIRONMENTA } \\
\text { LMONITORING }\end{array}$ & WAE & $\begin{array}{c}\prime \\
\text { DIXON, WILLI }\end{array}$ & DIXON, WILLIA & & 5 & & & $\begin{array}{l}\text { Benton } \\
\text { Washington }\end{array}$ & \\
\hline $\begin{array}{l}2614 W D \\
200 W\end{array}$ & $\begin{array}{l}\text { MONITORING } \\
\text { STATION }\end{array}$ & TRP & $\begin{array}{c}l \\
\text { PAYNE, MICH }\end{array}$ & $\begin{array}{l}01 / 44 \\
\text { HAMRICK, DOU }\end{array}$ & & & & & $\begin{array}{l}\text { Benton } \\
\text { Washington }\end{array}$ & \\
\hline $\begin{array}{l}267 Z \\
200 \mathrm{~W}\end{array}$ & $\begin{array}{l}\text { RISER \#9 VALVE } \\
\text { HOUSE }\end{array}$ & TRP & $\begin{array}{c}/ \\
\text { VOGT, ERIC C }\end{array}$ & $\begin{array}{l}06 / 88 \\
\text { VOGT, ERIC C }\end{array}$ & & & & & $\begin{array}{l}\text { Benton } \\
\text { Washington }\end{array}$ & \\
\hline $\begin{array}{r}2701 \mathrm{AB} \\
200 \mathrm{E}\end{array}$ & $\begin{array}{l}\text { PUREX BADGE } \\
\text { HOUSE }\end{array}$ & TRP & $\begin{array}{c}\text { ' } \\
\text { HAMRICK, DO }\end{array}$ & $\begin{array}{l}\text { 08/82 } \\
\text { HAMRICK, DOU }\end{array}$ & & 63 & & & $\begin{array}{l}\text { Benton } \\
\text { Washington }\end{array}$ & \\
\hline
\end{tabular}


WHC-SD-SQA-CSWD-501 Rev 0

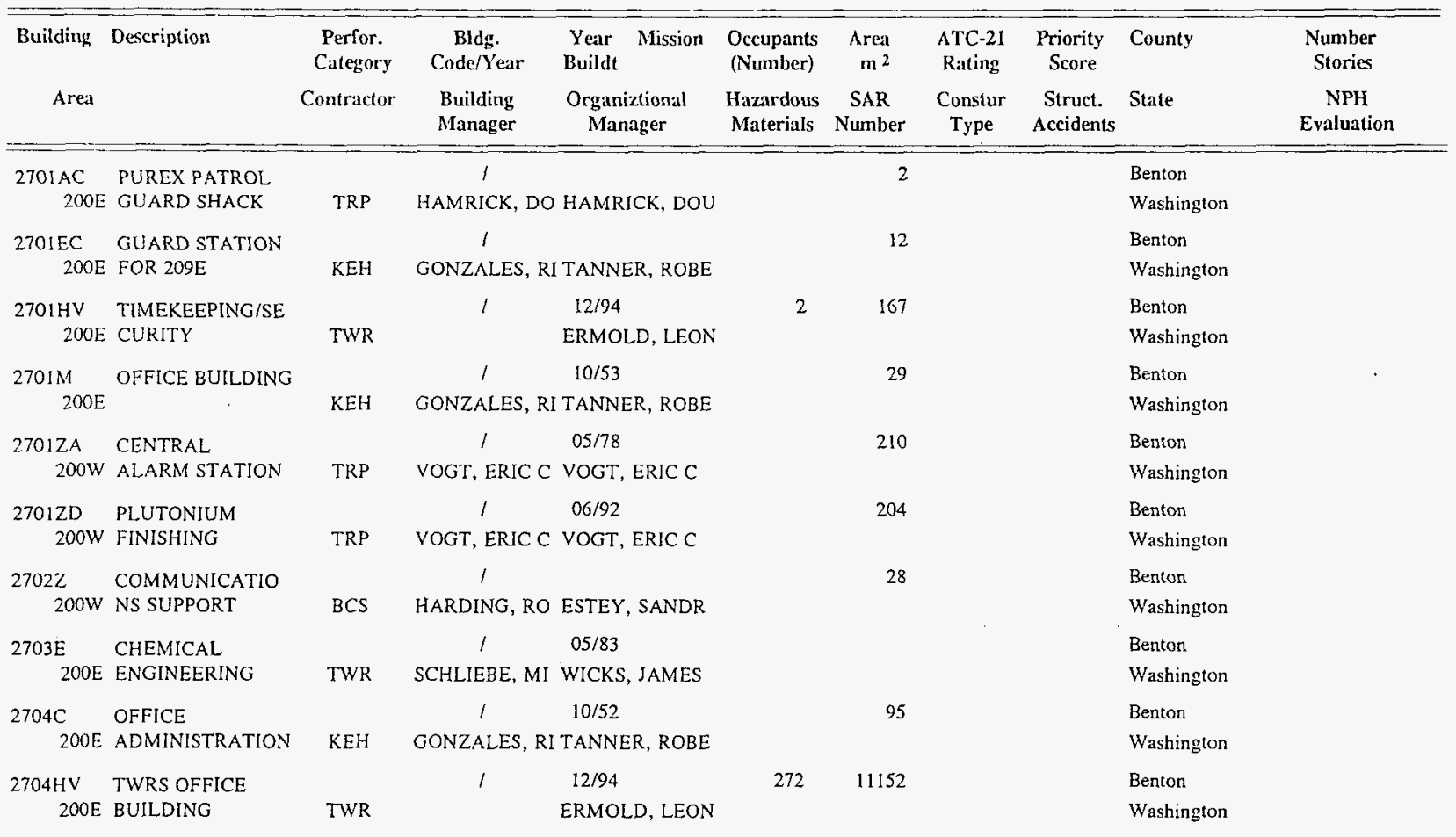


WHC-SD-SQA-CSWD-501 Rev 0

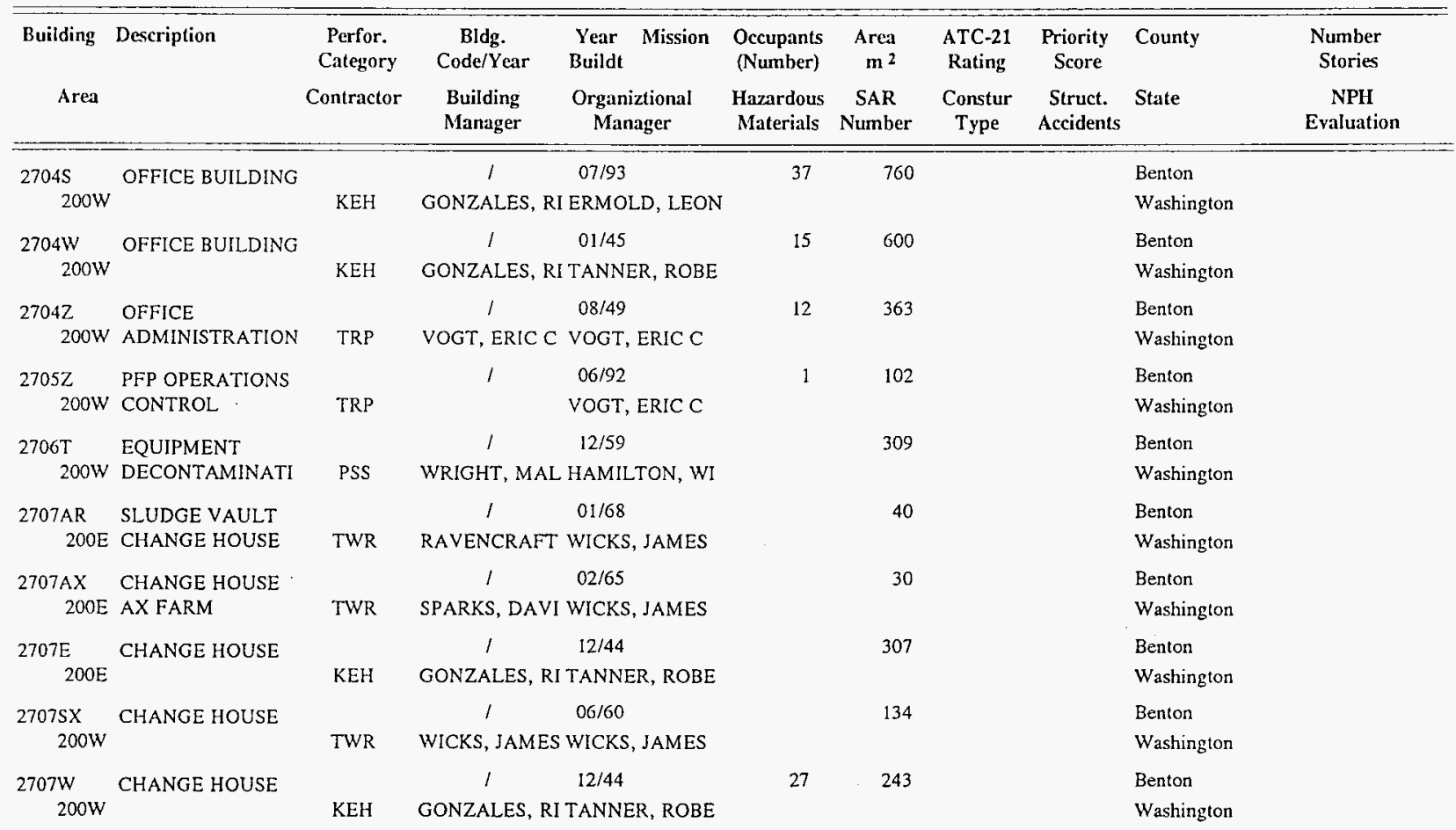


WHC-SD-SQA-CSWD-501 Rev 0

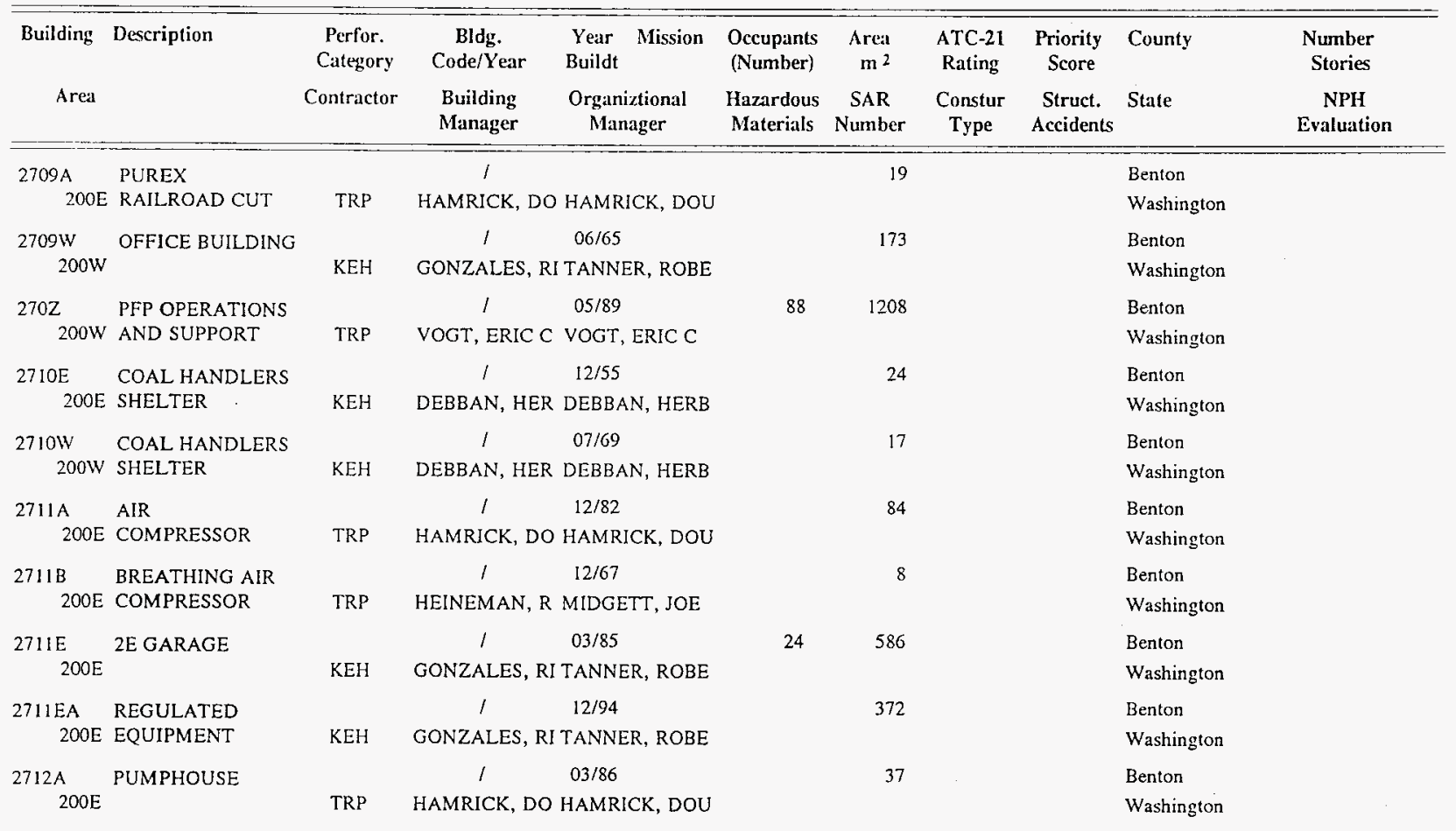


WHC-SD-SQA-CSWD-501 Rev 0

\begin{tabular}{|c|c|c|c|c|c|c|c|c|c|c|}
\hline Building & Description & $\begin{array}{l}\text { Perfor. } \\
\text { Category } \\
\text { Contractor }\end{array}$ & $\begin{array}{l}\text { Bldg. } \\
\text { Code/Year } \\
\text { Building } \\
\text { Manager }\end{array}$ & $\begin{array}{l}\text { Year Mission } \\
\text { Buildt } \\
\text { Organiztional } \\
\text { Manager }\end{array}$ & $\begin{array}{l}\text { Occupants } \\
\text { (Number) } \\
\text { Hazardous } \\
\text { Materials }\end{array}$ & $\begin{array}{c}\text { Area } \\
\mathrm{m}^{2} \\
\text { SAR } \\
\text { Number }\end{array}$ & $\begin{array}{l}\text { ATC-21 } \\
\text { Rating } \\
\text { Constur } \\
\text { Type }\end{array}$ & $\begin{array}{l}\text { Priority } \\
\text { Score } \\
\text { Struct. } \\
\text { Accidents }\end{array}$ & State & $\begin{array}{c}\text { Number } \\
\text { Stories } \\
\text { NPH } \\
\text { Evaluation }\end{array}$ \\
\hline $\begin{array}{l}2712 \mathrm{~B} \\
200 \mathrm{E}\end{array}$ & $\begin{array}{l}\text { ELECTRICAL } \\
\text { INSTRUMENTATI }\end{array}$ & TRP & REBER, DAVI & MIDGETT, JOE & & 2 & & & $\begin{array}{l}\text { Benton } \\
\text { Washington }\end{array}$ & \\
\hline $\begin{array}{l}2712 \mathrm{~T} \\
200 \mathrm{~W}\end{array}$ & $\begin{array}{l}\text { ELECTRICAL } \\
\text { INSTRUMENTATI }\end{array}$ & TWR & 1 & WICKS, JAMES & & & & & $\begin{array}{l}\text { Benton } \\
\text { Washington }\end{array}$ & \\
\hline $\begin{array}{l}2713 \mathrm{E} \\
200 \mathrm{E}\end{array}$ & $\begin{array}{l}\text { OFFICE } \\
\text { ADMINISTRATION }\end{array}$ & $\mathrm{KEH}$ & $\begin{array}{c}/ \\
\text { GONZALES, RI }\end{array}$ & $\begin{array}{l}12 / 44 \\
\text { TANNER, ROBE }\end{array}$ & & 384 & & & $\begin{array}{l}\text { Benton } \\
\text { Washington }\end{array}$ & \\
\hline $\begin{array}{l}2713 \mathrm{~W} \\
200 \mathrm{~W}\end{array}$ & GARAGE & PSS & $\begin{array}{c}/ \\
\text { GONZALES, RI }\end{array}$ & $\begin{array}{l}12 / 44 \\
\text { DILIBERTO, AN }\end{array}$ & 5 & 387 & & & $\begin{array}{l}\text { Benton } \\
\text { Washington }\end{array}$ & \\
\hline $\begin{array}{l}2713 \mathrm{WB} \\
200 \mathrm{~W}\end{array}$ & $\begin{array}{l}\text { REGULATED } \\
\text { GARAGE AND }\end{array}$ & TWR & $\begin{array}{c}\text { / } \\
\text { OWEN, PETER }\end{array}$ & $\begin{array}{c}12 / 44 \\
\text { WICKS, JAMES }\end{array}$ & & 572 & & & $\begin{array}{l}\text { Benton } \\
\text { Washington }\end{array}$ & \\
\hline $\begin{array}{l}2713 \mathrm{WC} \\
200 W\end{array}$ & $\begin{array}{l}\text { PESTICIDE } \\
\text { WASHWATER }\end{array}$ & $\mathrm{KEH}$ & 1 & DILIBERTO, AN & & 163 & & & $\begin{array}{l}\text { Benton } \\
\text { Washington }\end{array}$ & \\
\hline $\begin{array}{l}27\lfloor 4 \mathrm{~A} \\
200 \mathrm{E}\end{array}$ & $\begin{array}{l}\text { DRY CHEMICAL } \\
\text { WAREHOUSE }\end{array}$ & TRP & $\begin{array}{c}/ \\
\text { HAMRICK, DO }\end{array}$ & $\begin{array}{l}12 / 55 \\
\text { HAMRICK, DOU }\end{array}$ & & 209 & & & $\begin{array}{l}\text { Benton } \\
\text { Washington }\end{array}$ & \\
\hline $\begin{array}{l}2714 \mathrm{AR} \\
200 \mathrm{E}\end{array}$ & $\begin{array}{l}\text { SLUDGE VAULT } \\
\text { GENERAL }\end{array}$ & TWR & $\begin{array}{c}/ \\
\text { RAVENCRAFT }\end{array}$ & $\begin{array}{l}10 / 69 \\
\text { WICKS, JAMES }\end{array}$ & & 7 & & & $\begin{array}{l}\text { Benton } \\
\text { Washington }\end{array}$ & \\
\hline $\begin{array}{l}2714 \mathrm{U} \\
200 \mathrm{~W}\end{array}$ & WAREHOUSE & TRP & $\begin{array}{c}/ \\
\text { KAMMENZIN }\end{array}$ & $\begin{array}{l}04 / 52 \\
\text { HAMRICK, DOU }\end{array}$ & & 401 & & & $\begin{array}{l}\text { Benton } \\
\text { Washington }\end{array}$ & \\
\hline $\begin{array}{l}2715 \mathrm{~B} \\
200 \mathrm{E}\end{array}$ & $\begin{array}{l}\text { PAINT STORAGE } \\
\text { BUILDING }\end{array}$ & TRP & $\begin{array}{c}/ \\
\text { HEINEMAN, R }\end{array}$ & MIDGETT, JOE & & 24 & & & $\begin{array}{l}\text { Benton } \\
\text { Washington }\end{array}$ & \\
\hline
\end{tabular}


WHC-SD-SQA-CSWD-501 Rev 0

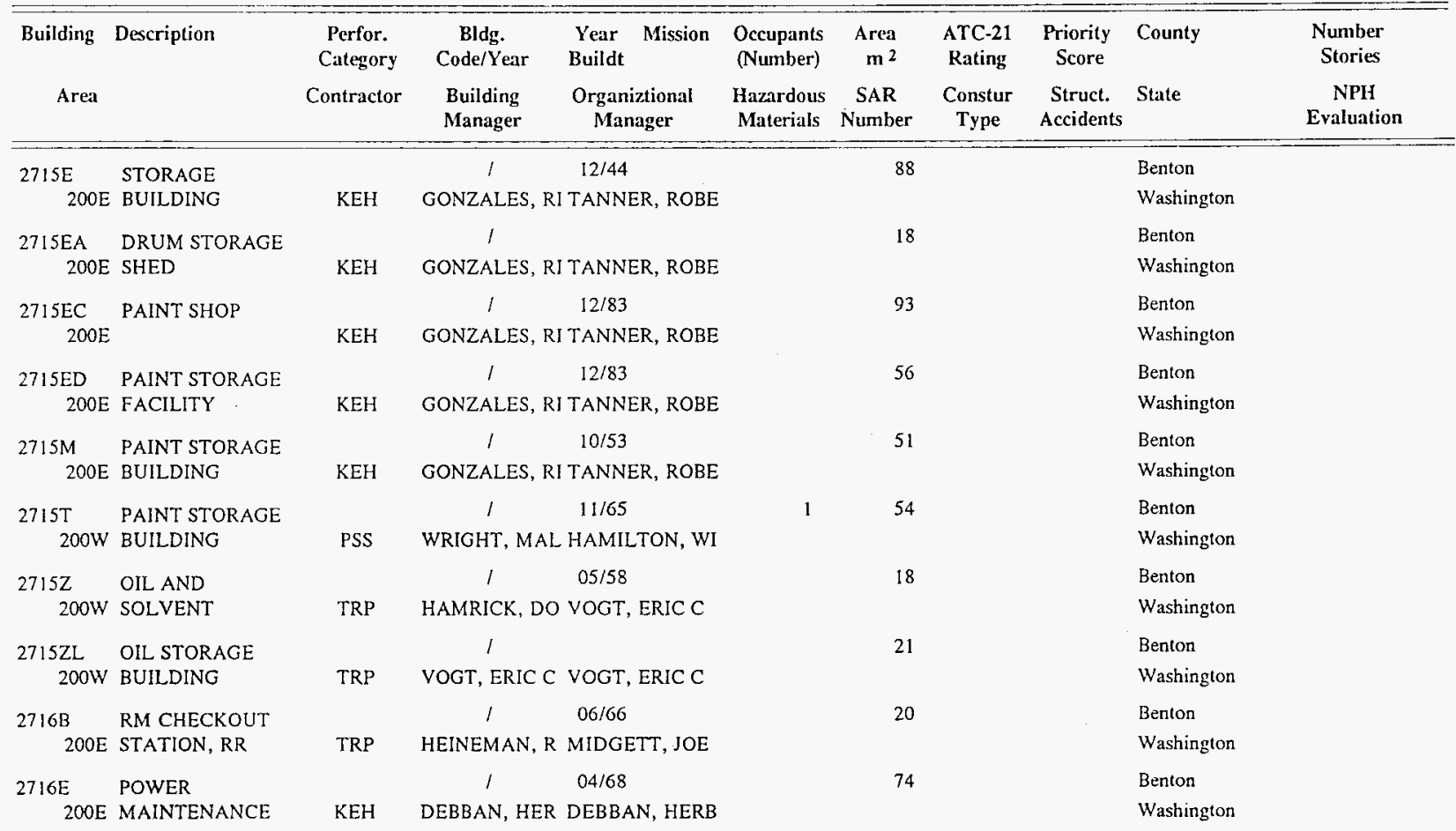


WHC-SD-SQA-CSWD-501 Rev 0

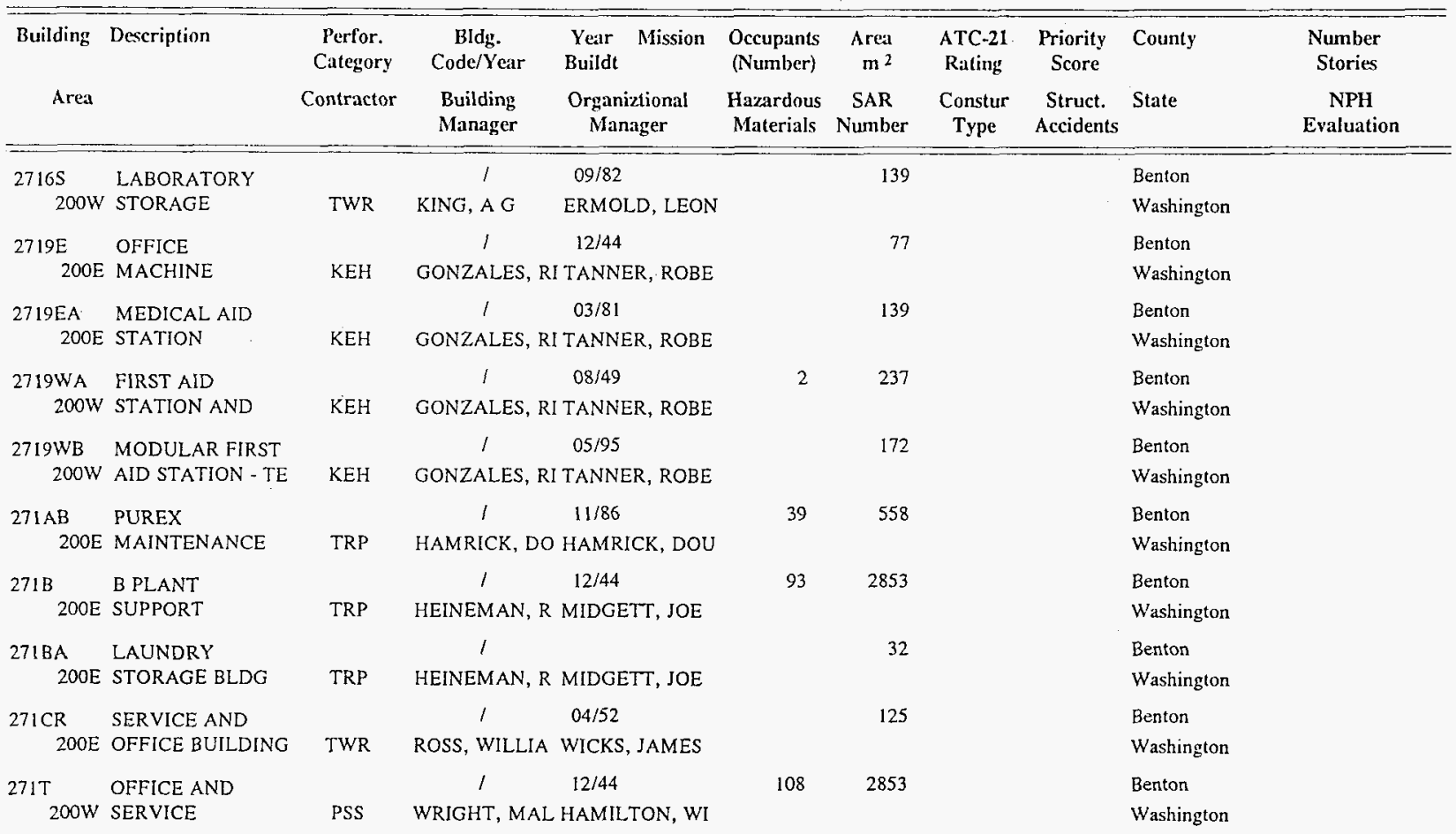


WHC-SD-SQA-CSWD-501 Rev 0

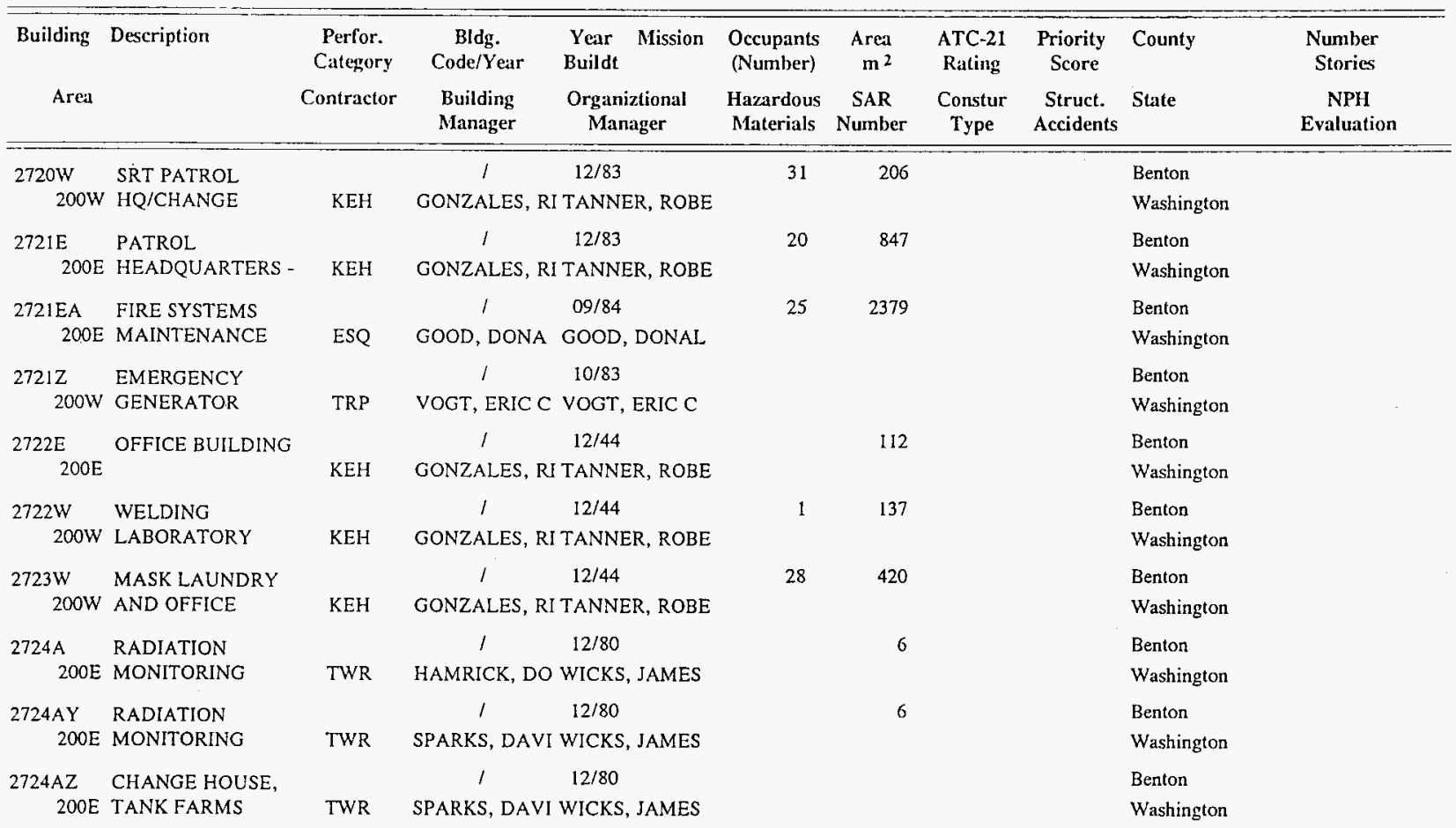


WHC-SD-SQA-CSWD-501 Rev 0

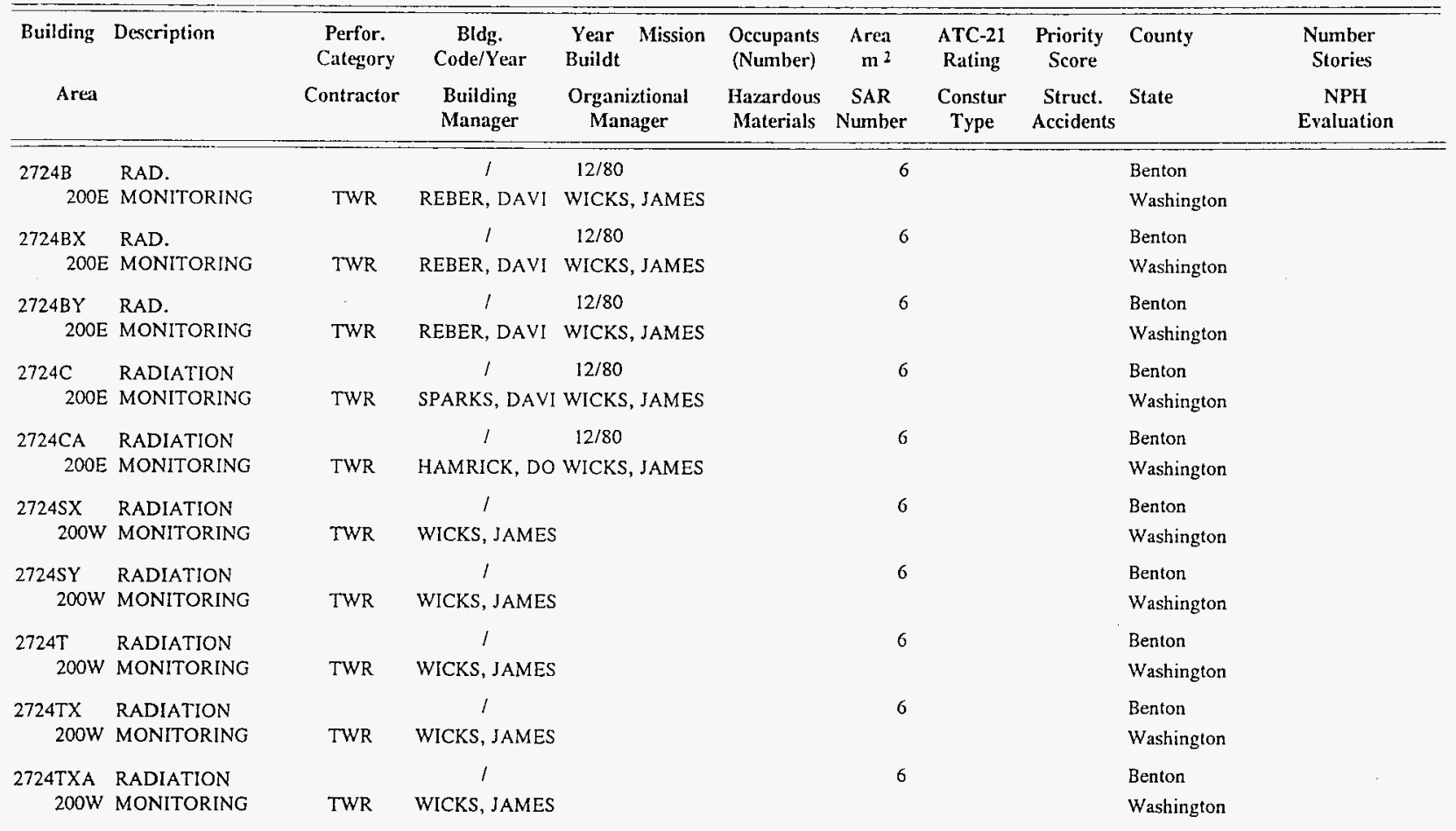


WHC-SD-SQA-CSWD-501 Rev 0

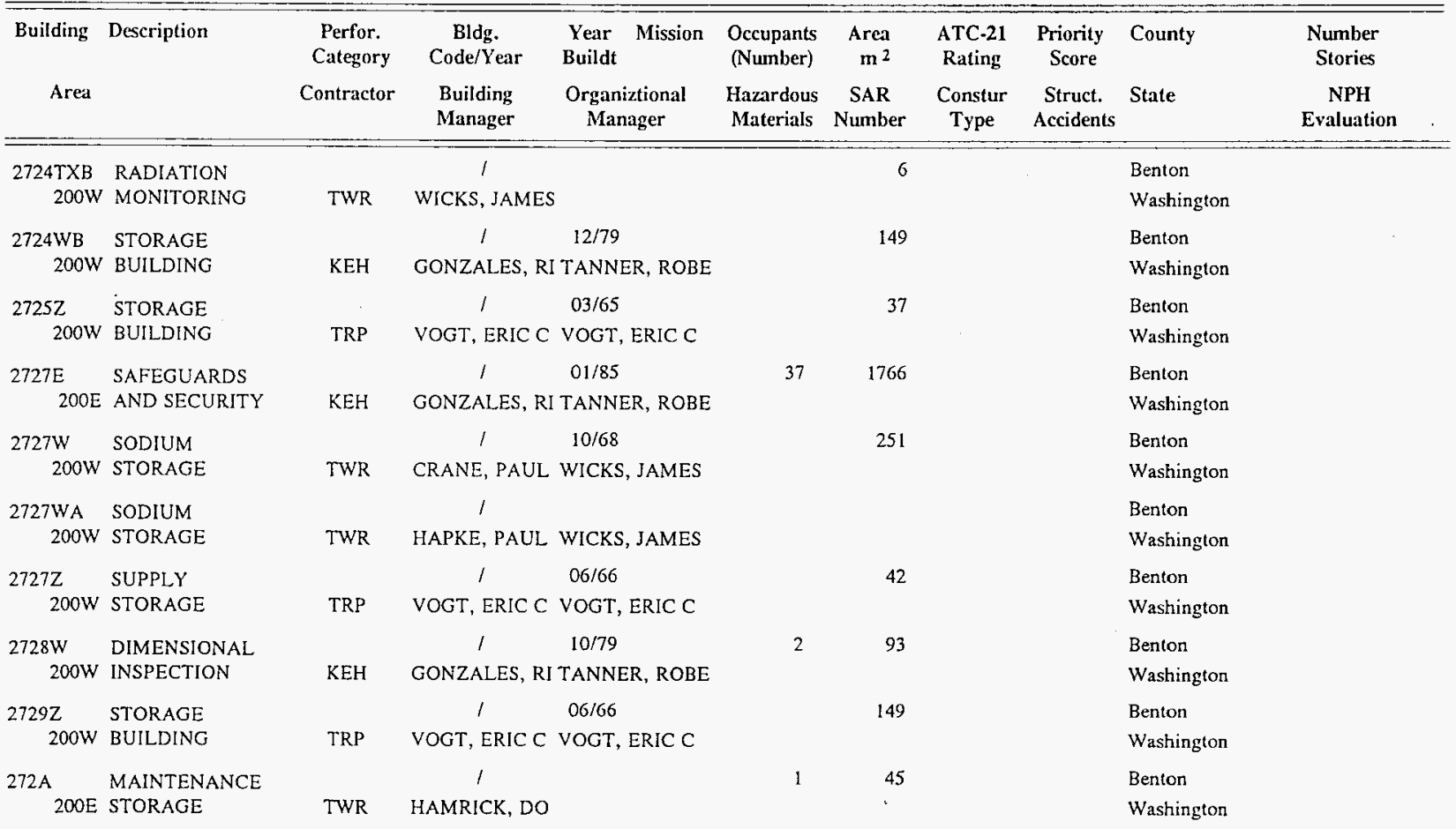


WHC-SD-SQA-CSWD-501 Rev 0

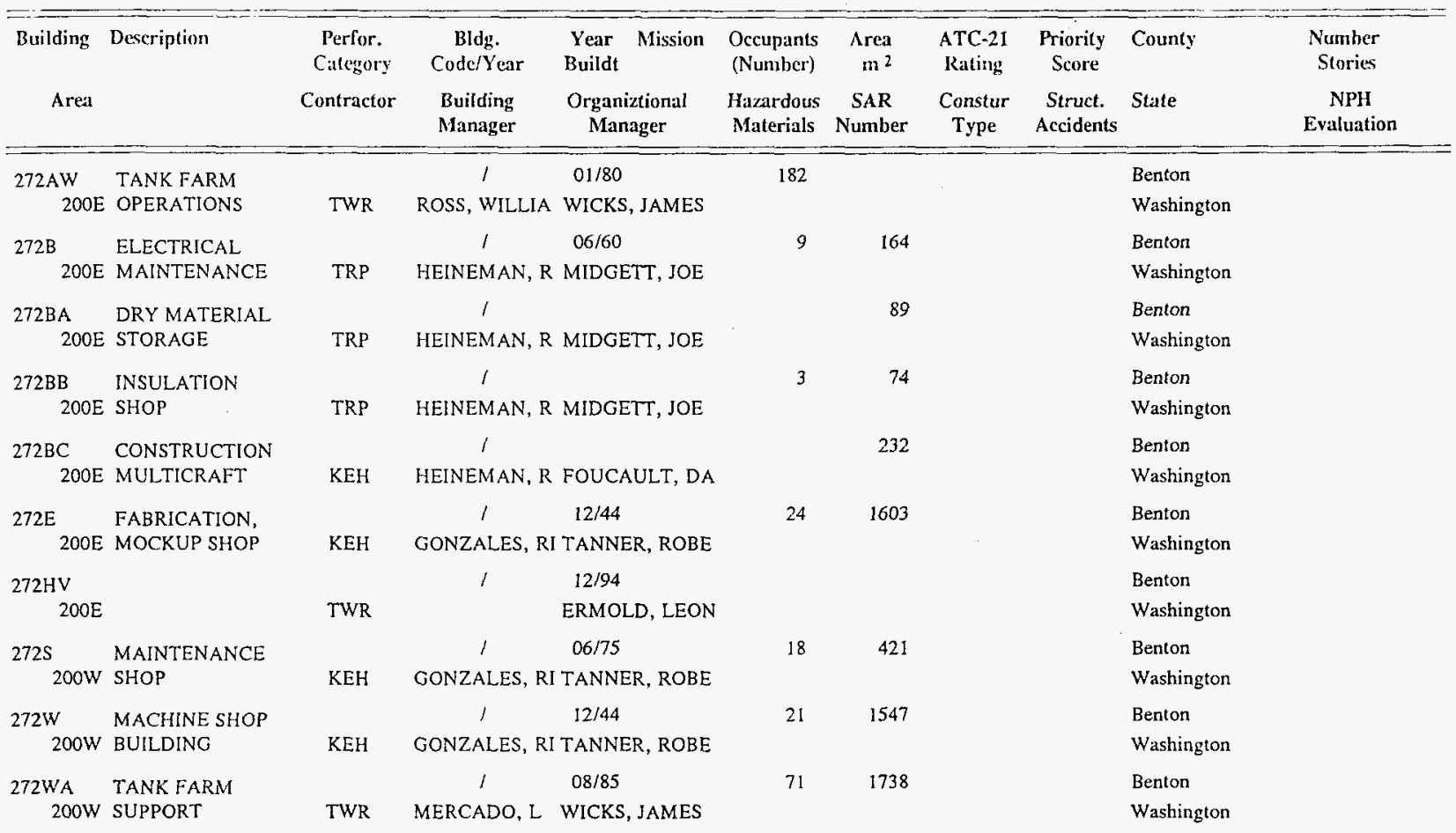


WHC-SD-SQA-CSWD-501 Rev 0

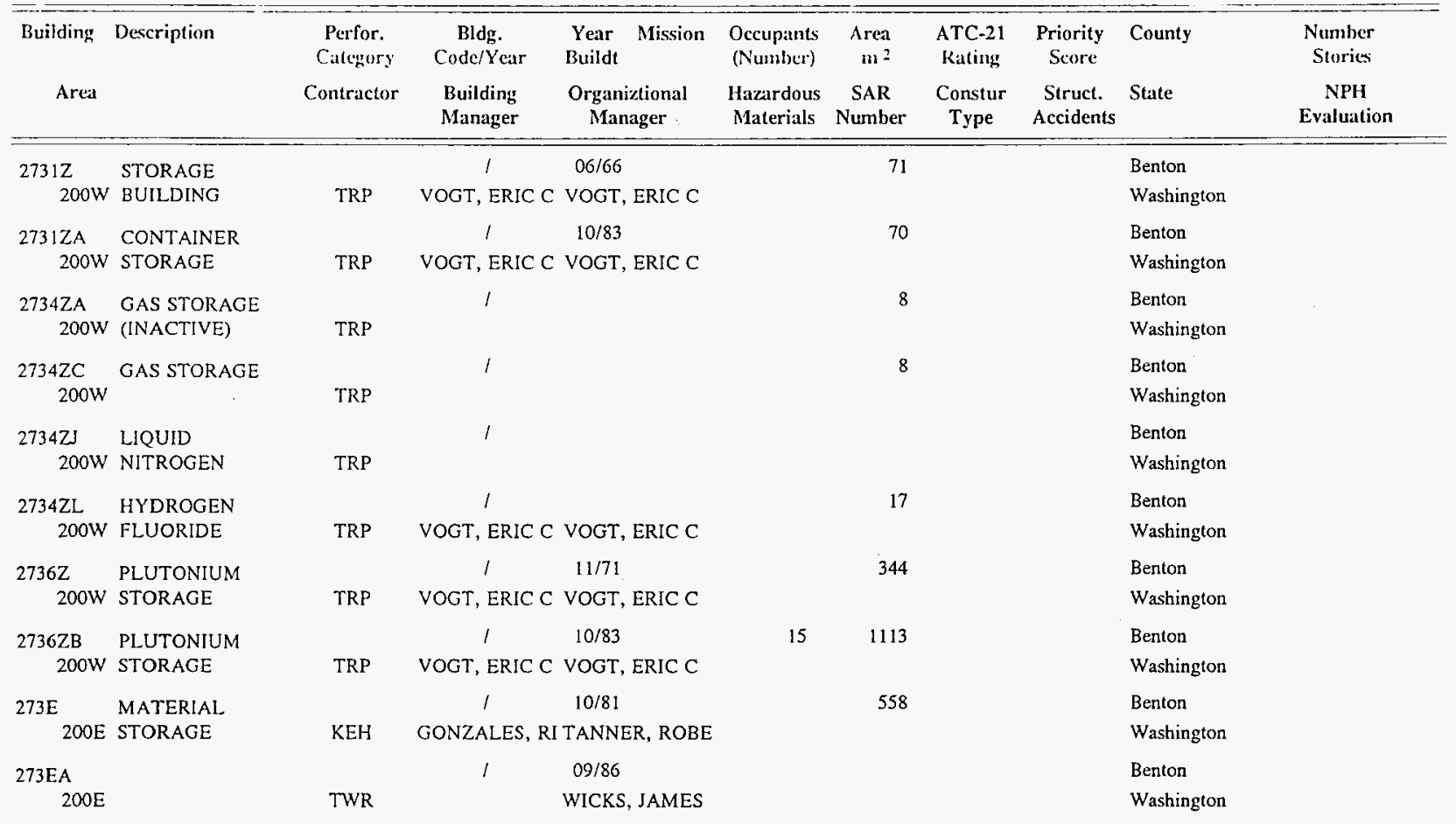


WHC-SD-SQA-CSWD-501 ReV 0

\begin{tabular}{|c|c|c|c|c|c|c|c|c|c|c|}
\hline Building & Description & $\begin{array}{l}\text { Perfor. } \\
\text { Category }\end{array}$ & $\begin{array}{c}\text { Bldg. } \\
\text { Code/Year }\end{array}$ & $\begin{array}{l}\text { Year Mission } \\
\text { Buildt }\end{array}$ & $\begin{array}{l}\text { Occupants } \\
\text { (Number) }\end{array}$ & $\begin{array}{r}\text { Area } \\
\mathbf{m}^{2}\end{array}$ & $\begin{array}{c}\text { ATC-21 } \\
\text { Rating }\end{array}$ & $\begin{array}{l}\text { Priority } \\
\text { Score }\end{array}$ & County & $\begin{array}{c}\text { Number } \\
\text { Stories }\end{array}$ \\
\hline Area & & Contractor & $\begin{array}{l}\text { Building } \\
\text { Manager }\end{array}$ & $\begin{array}{l}\text { Organiztional } \\
\text { Manager }\end{array}$ & $\begin{array}{l}\text { Hazardous } \\
\text { Materials }\end{array}$ & $\begin{array}{c}\text { SAR } \\
\text { Number }\end{array}$ & $\begin{array}{c}\text { Constur } \\
\text { Type }\end{array}$ & $\begin{array}{l}\text { Struct. } \\
\text { Accidents }\end{array}$ & State & $\begin{array}{c}\text { NPH } \\
\text { Evaluation }\end{array}$ \\
\hline $\begin{array}{l}273 \mathrm{~W} \\
200 \mathrm{~W}\end{array}$ & $\begin{array}{l}\text { MATERIAL } \\
\text { STORAGE }\end{array}$ & $\mathrm{KEH}$ & $\begin{array}{c}\prime \\
\text { GONZALES, }\end{array}$ & $\begin{array}{l}\text { 10/8 } \\
\text { I TANNER, ROBE }\end{array}$ & & 558 & & & $\begin{array}{l}\text { Benton } \\
\text { Washington }\end{array}$ & \\
\hline $\begin{array}{l}274 \mathrm{AW} \\
200 \mathrm{E}\end{array}$ & OFFICE BUILDING & $\mathrm{KEH}$ & $\begin{array}{c}\prime \\
\text { GONZALES, }\end{array}$ & $\begin{array}{l}04 / 94 \\
\text { I WICKS, JAMES }\end{array}$ & 41 & 836 & & & $\begin{array}{l}\text { Benton } \\
\text { Washington }\end{array}$ & \\
\hline${ }_{200 E}^{274 E}$ & $\begin{array}{l}\text { LANDLORD AND } \\
\text { MAINTENANCE }\end{array}$ & $\mathrm{KEH}$ & $\begin{array}{c}\prime \\
\text { GONZALES, }\end{array}$ & $\begin{array}{l}12 / 44 \\
\text { I TANNER, ROBE }\end{array}$ & 49 & 352 & & & $\begin{array}{l}\text { Benton } \\
\text { Washington }\end{array}$ & \\
\hline $\begin{array}{l}274 \mathrm{~W} \\
200 \mathrm{~W}\end{array}$ & OFFICE BUILDING & $\mathrm{KEH}$ & $\begin{array}{c}l \\
\text { GONZALES, }\end{array}$ & $\begin{array}{l}01 / 45 \\
\text { TANNER, ROBE }\end{array}$ & & 355 & & & $\begin{array}{l}\text { Benton } \\
\text { Washington }\end{array}$ & \\
\hline $\begin{array}{l}2750 \mathrm{E} \\
200 \mathrm{E}\end{array}$ & $\begin{array}{l}\text { WASTE } \\
\text { MANAGEMENT }\end{array}$ & $\mathrm{KEH}$ & $\begin{array}{c}\prime \\
\text { GONZALES, }\end{array}$ & $\begin{array}{l}07 / 80 \\
\text { TANNER, ROBE }\end{array}$ & 307 & 8922 & & & $\begin{array}{l}\text { Benton } \\
\text { Washington }\end{array}$ & \\
\hline $\begin{array}{l}2751 \mathrm{E} \\
200 \mathrm{E}\end{array}$ & OFFICE FACILITY & $\mathrm{KEH}$ & $\begin{array}{c}l \\
\text { GONZALES, }\end{array}$ & $\begin{array}{l}06 / 85 \\
\text { TANNER, ROBE }\end{array}$ & 56 & 1394 & & & $\begin{array}{l}\text { Benton } \\
\text { Washington }\end{array}$ & \\
\hline $\begin{array}{l}2752 \mathrm{E} \\
200 \mathrm{E}\end{array}$ & 200 EAST OFFICE & KEH & $\begin{array}{c}l \\
\text { GONZALES, }\end{array}$ & $\begin{array}{l}07 / 85 \\
\text { TANNER, ROBE }\end{array}$ & 56 & 1394 & & & $\begin{array}{l}\text { Benton } \\
\text { Washington }\end{array}$ & \\
\hline $\begin{array}{l}2753 \mathrm{E} \\
200 \mathrm{E}\end{array}$ & $\begin{array}{l}\text { OFFICE } \\
\text { BUILDING WIO }\end{array}$ & $\mathrm{KEH}$ & $\begin{array}{c}\prime \\
\text { GONZALES, }\end{array}$ & $\begin{array}{l}06 / 86 \\
\text { I TANNER, ROBE }\end{array}$ & 43 & 1394 & & & $\begin{array}{l}\text { Benton } \\
\text { Washington }\end{array}$ & \\
\hline${ }_{200 E}^{275 E}$ & $\begin{array}{l}\text { CARPENTER } \\
\text { SHOP BUILDING }\end{array}$ & $\mathrm{KEH}$ & $\begin{array}{c}\prime \\
\text { GONZALES, }\end{array}$ & $\begin{array}{l}12 / 44 \\
\text { TANNER, ROBE }\end{array}$ & 11 & 352 & & & $\begin{array}{l}\text { Benton } \\
\text { Washington }\end{array}$ & \\
\hline $\begin{array}{l}275 \mathrm{EA} \\
200 \mathrm{E}\end{array}$ & $\begin{array}{l}\text { WAREHOUSE } \\
\text { ESSENTIAL }\end{array}$ & $\mathrm{KEH}$ & $\begin{array}{c}/ \\
\text { GONZALES, }\end{array}$ & $\begin{array}{l}12 / 55 \\
\text { TANNER, ROBE }\end{array}$ & & 3346 & & & $\begin{array}{l}\text { Benton } \\
\text { Washington }\end{array}$ & \\
\hline
\end{tabular}


WHC-SD-SQA-CSWD-501 Rev 0

\begin{tabular}{|c|c|c|c|c|c|c|c|c|c|c|}
\hline Building & Description & $\begin{array}{c}\text { Perfor. } \\
\text { Category } \\
\text { Contractor }\end{array}$ & $\begin{array}{c}\text { Bldg. } \\
\text { Code/Year } \\
\text { Building } \\
\text { Manager }\end{array}$ & $\begin{array}{l}\text { Year Mission } \\
\text { Buildt } \\
\text { Organiztional } \\
\text { Manager }\end{array}$ & $\begin{array}{l}\text { Occupants } \\
\text { (Number) } \\
\text { Hazardous } \\
\text { Materials }\end{array}$ & $\begin{array}{c}\text { Area } \\
\text { m }^{2} \\
\text { SAR } \\
\text { Number }\end{array}$ & $\begin{array}{l}\text { ATC-21 } \\
\text { Rating } \\
\text { Constur } \\
\text { Type }\end{array}$ & $\begin{array}{l}\text { Priority } \\
\text { Score } \\
\text { Struct. } \\
\text { Accidents }\end{array}$ & State & $\begin{array}{c}\text { Number } \\
\text { Stories } \\
\text { NPH } \\
\text { Evaluation }\end{array}$ \\
\hline $\begin{array}{l}\text { 275UR } \\
\text { 200W }\end{array}$ & $\begin{array}{l}\text { CHEMICAL } \\
\text { STORAGE }\end{array}$ & KEH & $\begin{array}{c}\prime \\
\text { GONZALES, }\end{array}$ & $\begin{array}{l}04 / 52 \\
\text { I TANNER, ROBE }\end{array}$ & 3 & 268 & & & $\begin{array}{l}\text { Benton } \\
\text { Washington }\end{array}$ & \\
\hline $\begin{array}{l}275 \mathrm{~W} \\
200 \mathrm{~W}\end{array}$ & $\begin{array}{l}\text { HEAVY } \\
\text { EQUIPMENT SHOP }\end{array}$ & $\mathrm{KEH}$ & $\begin{array}{c}\text { / } \\
\text { GONZALES, }\end{array}$ & $\begin{array}{l}12 / 44 \\
\text { I TANNER, ROBE }\end{array}$ & 14 & 352 & & & $\begin{array}{l}\text { Benton } \\
\text { Washington }\end{array}$ & \\
\hline $276 \mathrm{~B}$ & PAINT SHOP & TRP & $\begin{array}{c}/ \\
\text { HEINEMAN, }\end{array}$ & $\begin{array}{l}12 / 67 \\
\text { MIDGETT, JOE }\end{array}$ & & 52 & & & $\begin{array}{l}\text { Benton } \\
\text { Washington }\end{array}$ & \\
\hline $\begin{array}{l}277 \mathrm{~A} \\
200 \mathrm{E}\end{array}$ & $\begin{array}{l}\text { ALL-CRAFT FAB } \\
\text { SHOP }\end{array}$ & PSS & 1 & $\begin{array}{l}11 / 94 \\
\text { KELLY, JAMES }\end{array}$ & & 297 & & & $\begin{array}{l}\text { Benton } \\
\text { Washington }\end{array}$ & \\
\hline${ }_{200 \mathrm{~W}}^{277 \mathrm{~T}}$ & $\begin{array}{l}\text { BLOW DOWN } \\
\text { BUILDING }\end{array}$ & PSS & $\begin{array}{c}\text { I } \\
\text { WRIGHT, MA }\end{array}$ & HAMILTON, WI & . & 112 & & & $\begin{array}{l}\text { Benton } \\
\text { Washington }\end{array}$ & \\
\hline $\begin{array}{l}277 \mathrm{~W} \\
200 \mathrm{~W}\end{array}$ & $\begin{array}{l}\text { FABRICATION } \\
\text { SHOP }\end{array}$ & $\mathrm{KEH}$ & $\begin{array}{c}l \\
\text { GONZALES, }\end{array}$ & $\begin{array}{l}02 / 52 \\
\text { TANNER, ROBE }\end{array}$ & 23 & 2711 & & & $\begin{array}{l}\text { Benton } \\
\text { Washington }\end{array}$ & \\
\hline $\begin{array}{r}278 \mathrm{AW} \\
200 \mathrm{E}\end{array}$ & $\begin{array}{l}\text { TANK FARM } \\
\text { DOCUMENT }\end{array}$ & $\mathrm{KEH}$ & $\begin{array}{c}l \\
\text { GONZALES, } R\end{array}$ & $\begin{array}{l}02 / 94 \\
\text { WICKS, JAMES }\end{array}$ & 5 & 172 & & & $\begin{array}{l}\text { Benton } \\
\text { Washington }\end{array}$ & \\
\hline $\begin{array}{l}278 \mathrm{WA} \\
200 \mathrm{~W}\end{array}$ & $\begin{array}{l}\text { TANK FARM } \\
\text { DOCUMENT }\end{array}$ & TWR & $\begin{array}{c}l \\
\text { GONZALES, }\end{array}$ & $\begin{array}{l}12 / 93 \\
\text { WICKS, JAMES }\end{array}$ & 25 & 172 & & & $\begin{array}{l}\text { Benton } \\
\text { Washington }\end{array}$ & \\
\hline $\begin{array}{l}281 \mathrm{~A} \\
200 \mathrm{E}\end{array}$ & $\begin{array}{l}\text { BACKUP } \\
\text { GENERATOR }\end{array}$ & TRP & $\begin{array}{c}/ \\
\text { HAMRICK, DC }\end{array}$ & $\begin{array}{l}08 / 83 \\
\text { HAMRICK, DOU }\end{array}$ & & 134 & & & $\begin{array}{l}\text { Benton } \\
\text { Washington }\end{array}$ & \\
\hline${ }^{282 B} 200 E$ & $\begin{array}{l}\text { WATER PUMP } \\
\text { HOUSE SOUTH }\end{array}$ & TRP & $\begin{array}{c}\text { । } \\
\text { HEINEMAN, }\end{array}$ & $\begin{array}{l}\text { 01/63 } \\
\text { MIDGETT, JOE }\end{array}$ & & 21 & & & $\begin{array}{l}\text { Benton } \\
\text { Washington }\end{array}$ & \\
\hline
\end{tabular}


WHC-SD-SQA-CSWD-501 Rev 0

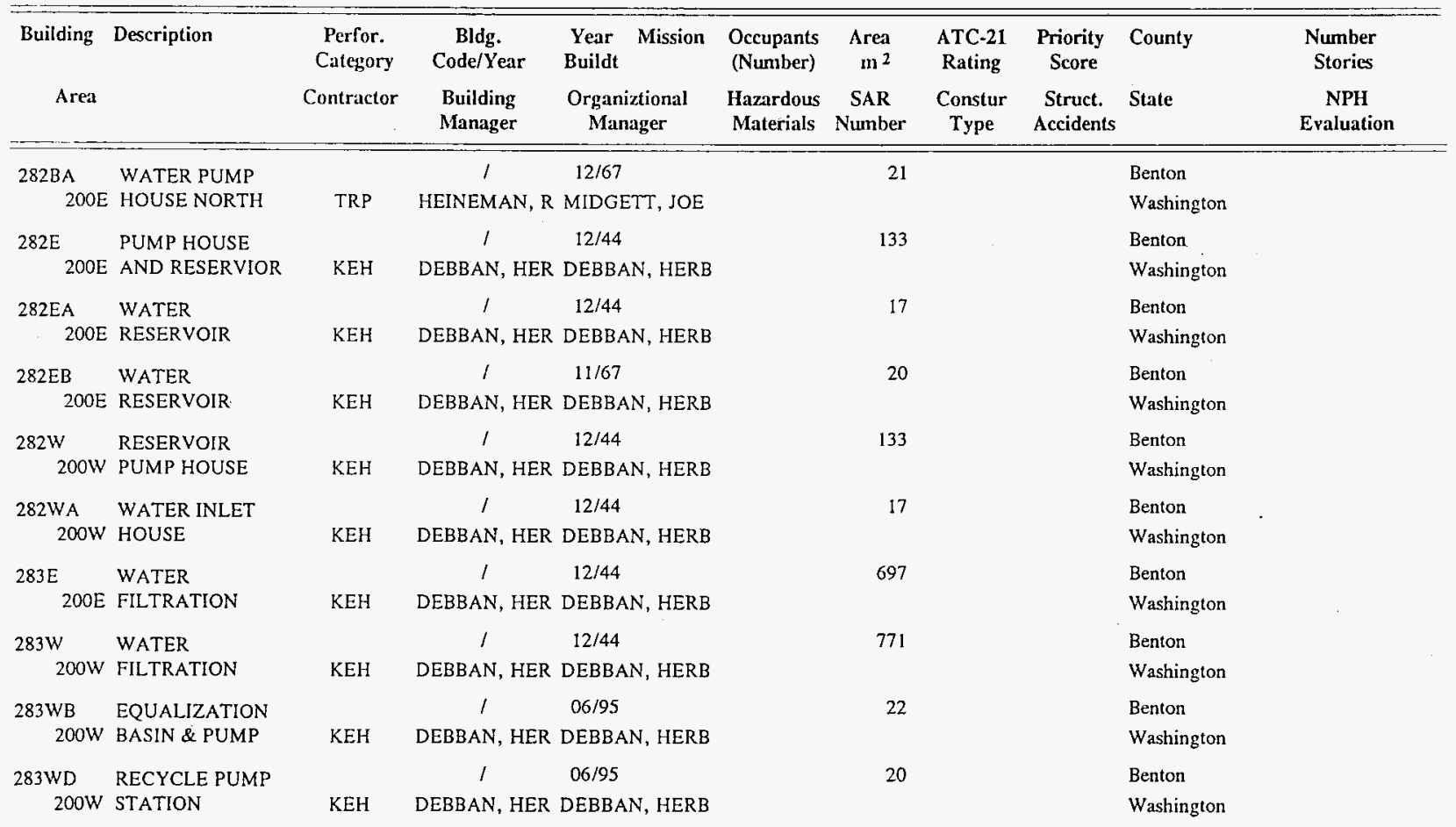


WHC-SD-SQA-CSWD-501 Rev 0

\begin{tabular}{|c|c|c|c|c|c|c|c|c|c|c|}
\hline Area & Description & $\begin{array}{c}\text { Perfor. } \\
\text { Category } \\
\text { Contractor }\end{array}$ & $\begin{array}{c}\text { Bldg. } \\
\text { Code/Year } \\
\text { Building } \\
\text { Manager }\end{array}$ & $\begin{array}{l}\text { Year Mission } \\
\text { Buildt } \\
\text { Organiztional } \\
\text { Manager }\end{array}$ & $\begin{array}{c}\text { Occupants } \\
\text { (Number) } \\
\text { Hazardous } \\
\text { Materials }\end{array}$ & $\begin{array}{c}\text { Area } \\
\text { m }^{2} \\
\text { SAR } \\
\text { Number }\end{array}$ & $\begin{array}{c}\text { ATC-21 } \\
\text { Rating } \\
\text { Constur } \\
\text { Type }\end{array}$ & $\begin{array}{l}\text { Priority } \\
\text { Score } \\
\text { Struct. } \\
\text { Accidents }\end{array}$ & State & $\begin{array}{c}\text { Number } \\
\text { Stories } \\
\text { NPH } \\
\text { Evaluation }\end{array}$ \\
\hline $\begin{array}{l}\text { 283WE } \\
200 W\end{array}$ & $\begin{array}{l}\text { SLUDGE } \\
\text { LAGOONS }\end{array}$ & $\mathrm{KEH}$ & I & & & 2509 & & & $\begin{array}{l}\text { Benton } \\
\text { Washington }\end{array}$ & \\
\hline $\begin{array}{l}283 W F \\
200 W\end{array}$ & $\begin{array}{l}\text { SAMPLE } \\
\text { BUILDING }\end{array}$ & $\mathrm{KEH}$ & $\begin{array}{c}/ \\
\text { DEBBAN, HER }\end{array}$ & $\begin{array}{l}06 / 95 \\
\text { DEBBAN, HERB }\end{array}$ & & 16 & & & $\begin{array}{l}\text { Benton } \\
\text { Washington }\end{array}$ & \\
\hline $\begin{array}{l}284 \mathrm{E} \\
200 \mathrm{E}\end{array}$ & $\begin{array}{l}\text { POWER HOUSE } \\
\text { AND STEAM }\end{array}$ & $\mathrm{KEH}$ & $\begin{array}{c}l \\
\text { DEBBAN, HER }\end{array}$ & $\begin{array}{l}12 / 44 \\
\text { DEBBAN, HERB }\end{array}$ & 41 & 3143 & & & $\begin{array}{l}\text { Benton } \\
\text { Washington }\end{array}$ & \\
\hline $\begin{array}{l}284 \mathrm{~W} \\
200 \mathrm{~W}\end{array}$ & $\begin{array}{l}\text { POWER HOUSE } \\
\text { STEAM PLANT }\end{array}$ & KEH & $\begin{array}{c}/ \\
\text { DEBBAN, HER }\end{array}$ & $\begin{array}{c}12 / 44 \\
\text { DEBBAN, HERB }\end{array}$ & & 2522 & & & $\begin{array}{l}\text { Benton } \\
\text { Washington }\end{array}$ & \\
\hline $\begin{array}{l}284 W B \\
200 W\end{array}$ & $\begin{array}{l}\text { PACKAGE } \\
\text { BOILER PLANT }\end{array}$ & KEH & 1 & & & 158 & & & $\begin{array}{l}\text { Benton } \\
\text { Washington }\end{array}$ & \\
\hline $\begin{array}{l}285 \mathrm{~W} \\
200 \mathrm{~W}\end{array}$ & $\begin{array}{l}\text { BACKFLOW } \\
\text { PREVENTION }\end{array}$ & PSS & $\begin{array}{c}/ \\
\text { DEBBAN, HER }\end{array}$ & & & 9 & & & $\begin{array}{l}\text { Benton } \\
\text { Washington }\end{array}$ & \\
\hline $\begin{array}{l}286 \mathrm{~W} \\
200 \mathrm{~W}\end{array}$ & $\begin{array}{l}\text { RMW BACKFLOW } \\
\text { PREVENTER }\end{array}$ & PSS & $\begin{array}{c}\text { I } \\
\text { CRANE, PAUL }\end{array}$ & $\begin{array}{l}10 / 92 \\
\text { HAMILTON, WI }\end{array}$ & & 9 & & & $\begin{array}{l}\text { Benton } \\
\text { Washington }\end{array}$ & \\
\hline $\begin{array}{l}2901 Y \\
600\end{array}$ & $\begin{array}{l}\text { EXPORT \& LINE } \\
\text { VALVE HOSE, }\end{array}$ & $\mathrm{KEH}$ & 1 & & & 28 & & & $\begin{array}{l}\text { Benton } \\
\text { Washington }\end{array}$ & \\
\hline $\begin{array}{l}2902 \mathrm{HV} \\
200 \mathrm{E}\end{array}$ & & TWR & 1 & $\begin{array}{l}12 / 94 \\
\text { ERMOLD, LEON }\end{array}$ & & & & & $\begin{array}{l}\text { Benton } \\
\text { Washington }\end{array}$ & \\
\hline $\begin{array}{l}2904 \mathrm{ZA} \\
200 \mathrm{~W}\end{array}$ & $\begin{array}{l}\text { RADIATION AND } \\
\text { FLOW }\end{array}$ & TRP & $\begin{array}{c}l \\
\text { VOGT, ERIC C }\end{array}$ & VOGT, ERIC C & & 4 & & & $\begin{array}{l}\text { Benton } \\
\text { Washington }\end{array}$ & \\
\hline
\end{tabular}


WHC-SD-SQA-CSWD-501 Rev 0

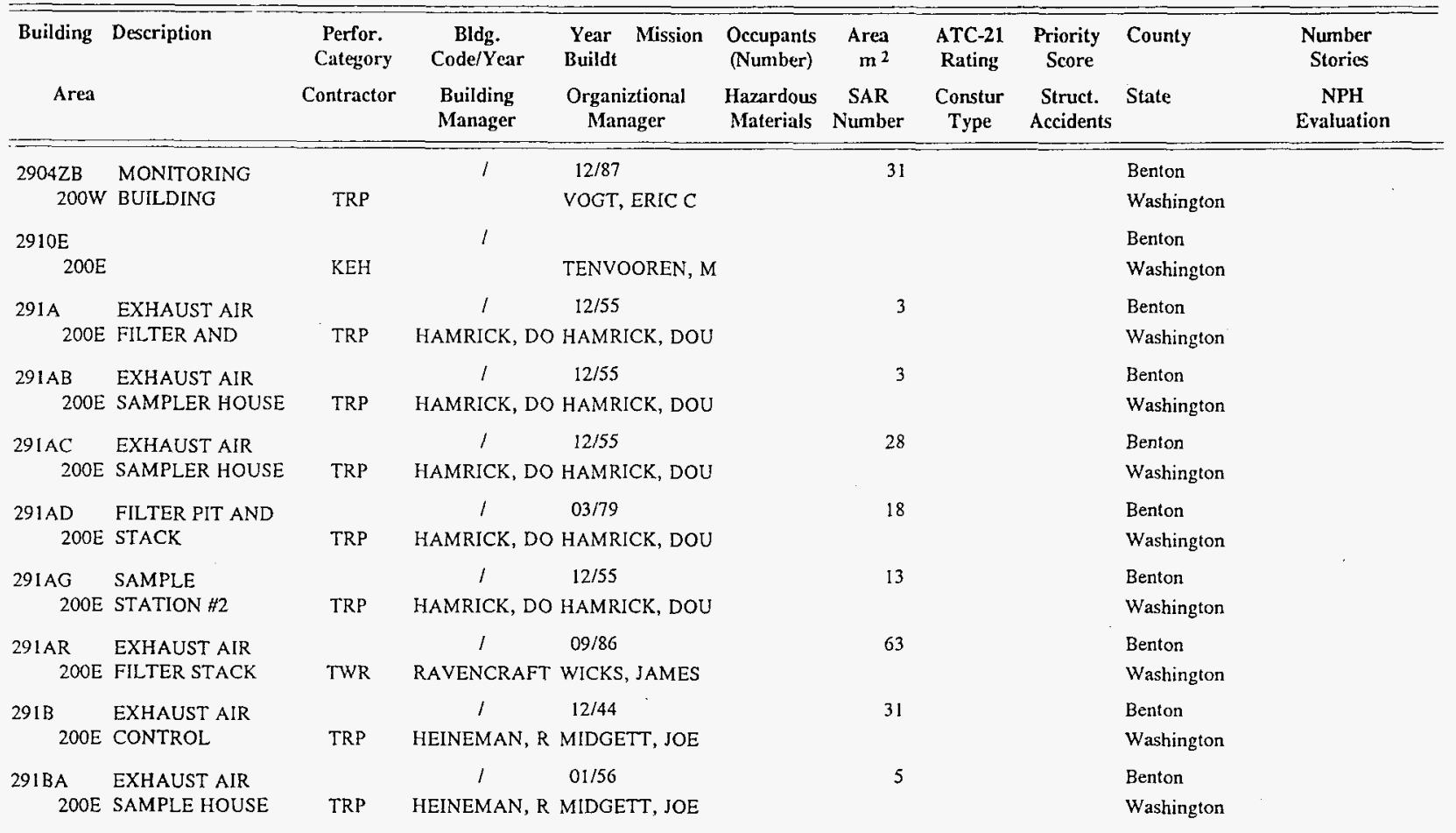


WHC-SD-SQA-CSWD-501 Rev 0

\begin{tabular}{|c|c|c|c|c|c|c|c|c|c|c|}
\hline Building & Description & $\begin{array}{l}\text { Perfor. } \\
\text { Category } \\
\text { Contractor }\end{array}$ & $\begin{array}{c}\text { Bldg. } \\
\text { Code/Year } \\
\text { Building } \\
\text { Manager }\end{array}$ & $\begin{array}{l}\text { Year Mission } \\
\text { Buildt } \\
\text { Organiztional } \\
\text { Manager }\end{array}$ & $\begin{array}{l}\text { Occupants } \\
\text { (Number) } \\
\text { Hazardous } \\
\text { Materials }\end{array}$ & $\begin{array}{c}\text { Area } \\
\mathrm{m}^{2} \\
\text { SAR } \\
\text { Number }\end{array}$ & $\begin{array}{l}\text { ATC-21 } \\
\text { Rating } \\
\text { Constur } \\
\text { Type }\end{array}$ & $\begin{array}{l}\text { Priority } \\
\text { Score } \\
\text { Struct. } \\
\text { Accidents }\end{array}$ & State & $\begin{array}{c}\text { Number } \\
\text { Stories } \\
\text { NPH } \\
\text { Evaluation }\end{array}$ \\
\hline $\begin{array}{l}29 \text { IBB } \\
200 E\end{array}$ & $\begin{array}{l}\text { INSTRUMENT } \\
\text { BUILDING - A\&B }\end{array}$ & TRP & $\begin{array}{c}l \\
\text { HEINEMAN, R }\end{array}$ & $\begin{array}{l}01 / 68 \\
\text { MIDGETT, JOE }\end{array}$ & & 27 & & & $\begin{array}{l}\text { Benton } \\
\text { Washington }\end{array}$ & \\
\hline $\begin{array}{l}291 \mathrm{BD} \\
200 \mathrm{E}\end{array}$ & $\begin{array}{l}\text { C FILTER \& } \\
\text { INSTRUMENT }\end{array}$ & TRP & $\begin{array}{c}l \\
\text { HEINEMAN, R }\end{array}$ & $\begin{array}{l}10 / 70 \\
\text { MIDGETT, JOE }\end{array}$ & & 101 & & & $\begin{array}{l}\text { Benton } \\
\text { Washington }\end{array}$ & \\
\hline $\begin{array}{l}291 \mathrm{BF} \\
200 \mathrm{E}\end{array}$ & D FILTER & TRP & $\begin{array}{c}/ \\
\text { HEINEMAN, R }\end{array}$ & $\begin{array}{l}\text { 06/79 } \\
\text { MIDGETT, JOE }\end{array}$ & & 42 & & & $\begin{array}{l}\text { Benton } \\
\text { Washington }\end{array}$ & \\
\hline $\begin{array}{l}291 \mathrm{BH} \\
200 \mathrm{E}\end{array}$ & E FILTER & TRP & $\begin{array}{c}l \\
\text { HEINEMAN, R }\end{array}$ & $\begin{array}{l}04 / 81 \\
\text { MIDGETT, JOE }\end{array}$ & & 9 & & & $\begin{array}{l}\text { Benton } \\
\text { Washington }\end{array}$ & \\
\hline $\begin{array}{l}291 \mathrm{BK} \\
200 \mathrm{E}\end{array}$ & $\begin{array}{l}\text { INSTRUMENT } \\
\text { BUILDING - E \& F }\end{array}$ & TRP & $\begin{array}{c}\text { / } \\
\text { HEINEMAN, R }\end{array}$ & $\begin{array}{l}10 / 93 \\
\text { MIDGETT, JOE }\end{array}$ & & 9 & & & $\begin{array}{l}\text { Benton } \\
\text { Washington }\end{array}$ & \\
\hline $\begin{array}{l}291 \mathrm{Z} \\
200 \mathrm{~W}\end{array}$ & $\begin{array}{l}\text { EXHAUST AIR } \\
\text { FILTER STACK }\end{array}$ & TRP & $\begin{array}{c}\prime \\
\text { VOGT, ERIC C }\end{array}$ & $\begin{array}{l}\text { 06/50 } \\
\text { VOGT, ERIC C }\end{array}$ & & 1271 & & & $\begin{array}{l}\text { Benton } \\
\text { Washington }\end{array}$ & \\
\hline $\begin{array}{l}2920 G W \\
\text { RCHN }\end{array}$ & BUILDING ETC2 & FDH & PHMC $^{\prime}$ & & 114 & & & & $\begin{array}{l}\text { Benton } \\
\text { Washington }\end{array}$ & \\
\hline $\begin{array}{l}292 \mathrm{AA} \\
200 \mathrm{E}\end{array}$ & $\begin{array}{l}\text { PR STACK } \\
\text { SAMPLE HOUSE }\end{array}$ & TRP & $\begin{array}{c}l \\
\text { HAMRICK, DO }\end{array}$ & $\begin{array}{l}11 / 81 \\
\text { HAMRICK, DOU }\end{array}$ & & & & & $\begin{array}{l}\text { Benton } \\
\text { Washington }\end{array}$ & \\
\hline $\begin{array}{l}292 \mathrm{AB} \\
200 \mathrm{E}\end{array}$ & $\begin{array}{l}\text { PUREX GASES } \\
\text { EFFLUENT }\end{array}$ & TRP & $\begin{array}{c}\text { I } \\
\text { HAMRICK, DO }\end{array}$ & HAMRICK, DOU & & 56 & & & $\begin{array}{l}\text { Benton } \\
\text { Washington }\end{array}$ & \\
\hline $\begin{array}{l}292 \mathrm{~B} \\
200 \mathrm{E}\end{array}$ & $\begin{array}{l}\text { STACK MONITOR } \\
\text { STATION }\end{array}$ & TRP & $\begin{array}{c}l \\
\text { HEINEMAN, R }\end{array}$ & $\begin{array}{l}01 / 49 \\
\text { MIDGETT, JOE }\end{array}$ & & 31 & & & $\begin{array}{l}\text { Benton } \\
\text { Washington }\end{array}$ & \\
\hline
\end{tabular}


WHC-SD-SQA-CSWD-501 Rev 0

\begin{tabular}{|c|c|c|c|c|c|c|c|c|c|c|}
\hline Building & Description & $\begin{array}{c}\text { Perfor. } \\
\text { Category } \\
\text { Contractor }\end{array}$ & $\begin{array}{c}\text { Bldg. } \\
\text { Code/Year } \\
\text { Building } \\
\text { Manager }\end{array}$ & $\begin{array}{l}\text { Year Mission } \\
\text { Buildt } \\
\text { Organiztional } \\
\text { Manager }\end{array}$ & $\begin{array}{c}\text { Occupants } \\
\text { (Number) } \\
\text { Hazardous } \\
\text { Materials }\end{array}$ & $\begin{array}{c}\begin{array}{c}\text { Area } \\
\mathrm{m}^{2}\end{array} \\
\text { SAR } \\
\text { Number }\end{array}$ & $\begin{array}{c}\text { ATC-21 } \\
\text { Rating } \\
\text { Constur } \\
\text { Type }\end{array}$ & $\begin{array}{l}\text { Priority } \\
\text { Score } \\
\text { Struct. } \\
\text { Accidents }\end{array}$ & State & $\begin{array}{c}\text { Number } \\
\text { Stories } \\
\text { NPH } \\
\text { Evaluation }\end{array}$ \\
\hline $\begin{array}{l}292 \mathrm{~T} \\
200 \mathrm{~W}\end{array}$ & $\begin{array}{l}\text { FISSION } \\
\text { PRODUCTS }\end{array}$ & PSS & $\begin{array}{c}\text { / } \\
\text { WRIGHT, MA }\end{array}$ & HAMILTON, WI & & 85 & & & $\begin{array}{l}\text { Benton } \\
\text { Washington }\end{array}$ & \\
\hline${ }_{200 E}^{293 A}$ & $\begin{array}{l}\text { OFFGAS } \\
\text { TREATMENT }\end{array}$ & TRP & $\begin{array}{c}\prime \\
\text { HAMRICK, D }\end{array}$ & $\begin{array}{l}\text { 09/57 } \\
\text { HAMRICK, DOU }\end{array}$ & & 175 & & & $\begin{array}{l}\text { Benton } \\
\text { Washington }\end{array}$ & \\
\hline $\begin{array}{l}2940 G W \\
\text { RCHN }\end{array}$ & BUILDING ETC1 & FDH & PHMC & 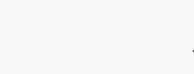 & 6 & & & & $\begin{array}{l}\text { Benton } \\
\text { Washington }\end{array}$ & \\
\hline $\begin{array}{l}294 \mathrm{~A} \\
200 \mathrm{E}\end{array}$ & $\begin{array}{l}\text { OFFGAS } \\
\text { TREATMENT }\end{array}$ & TRP & $\begin{array}{c}/ \\
\text { HAMRICK, D }\end{array}$ & $\begin{array}{l}04 / 63 \\
\text { HAMRICK, DOU }\end{array}$ & & 9 & & & $\begin{array}{l}\text { Benton } \\
\text { Washington }\end{array}$ & \\
\hline $\begin{array}{l}295 \mathrm{~A} \\
200 \mathrm{E}\end{array}$ & $\begin{array}{l}\text { ASD SAMPLE } \\
\text { STATION }\end{array}$ & TRP & $\begin{array}{c}/ \\
\text { HAMRICK, D }\end{array}$ & $\begin{array}{l}\text { 04/68 } \\
\text { HAMRICK, DOU }\end{array}$ & & 4 & & & $\begin{array}{l}\text { Benton } \\
\text { Washington }\end{array}$ & \\
\hline $\begin{array}{l}\text { 295AA } \\
200 \mathrm{E}\end{array}$ & $\begin{array}{l}\text { SCD SAMPLE } \\
\text { AND PUMPOUT }\end{array}$ & TRP & $\begin{array}{c}\prime \\
\text { HAMRICK, D }\end{array}$ & $\begin{array}{l}10 / 85 \\
\text { HAMRICK, DOU }\end{array}$ & & & & & $\begin{array}{l}\text { Benton } \\
\text { Washington }\end{array}$ & \\
\hline $\begin{array}{l}295 \mathrm{AC} \\
200 \mathrm{E}\end{array}$ & $\begin{array}{l}\text { CSL SAMPLE } \\
\text { STATION }\end{array}$ & TRP & $\begin{array}{c}I \\
\text { HAMRICK, D }\end{array}$ & $\begin{array}{l}12 / 81 \\
\text { HAMRICK, DOU }\end{array}$ & & & & & $\begin{array}{l}\text { Benton } \\
\text { Washington }\end{array}$ & \\
\hline $\begin{array}{l}295 \mathrm{AD} \\
200 \mathrm{E}\end{array}$ & $\begin{array}{l}\text { SWL SAMPLE } \\
\text { STATION }\end{array}$ & TWR & $\begin{array}{c}l \\
\text { HAMRICK, D }\end{array}$ & HAMRICK, DOU & & 13 & & & $\begin{array}{l}\text { Benton } \\
\text { Washington }\end{array}$ & \\
\hline $\begin{array}{l}295 \mathrm{AE} \\
200 \mathrm{E}\end{array}$ & $\begin{array}{l}\text { PDD } \\
\text { MONITORING }\end{array}$ & TRP & $\begin{array}{c}\prime \\
\text { HAMRICK, D }\end{array}$ & $\begin{array}{l}06 / 88 \\
\text { HAMRICK, DOU }\end{array}$ & & 28 & & & $\begin{array}{l}\text { Benton } \\
\text { Washington }\end{array}$ & \\
\hline $\begin{array}{r}296 \mathrm{HN} 29 \\
200 \mathrm{E}\end{array}$ & $\begin{array}{l}\text { STACK MONITOR } \\
\text { SAMPLE }\end{array}$ & TWR & 1 & $\begin{array}{l}12 / 85 \\
\text { WICKS, JAMES }\end{array}$ & & 112 & & & $\begin{array}{l}\text { Benton } \\
\text { Washington }\end{array}$ & \\
\hline
\end{tabular}


WHC-SD-SQA-CSWD-501 Rev 0

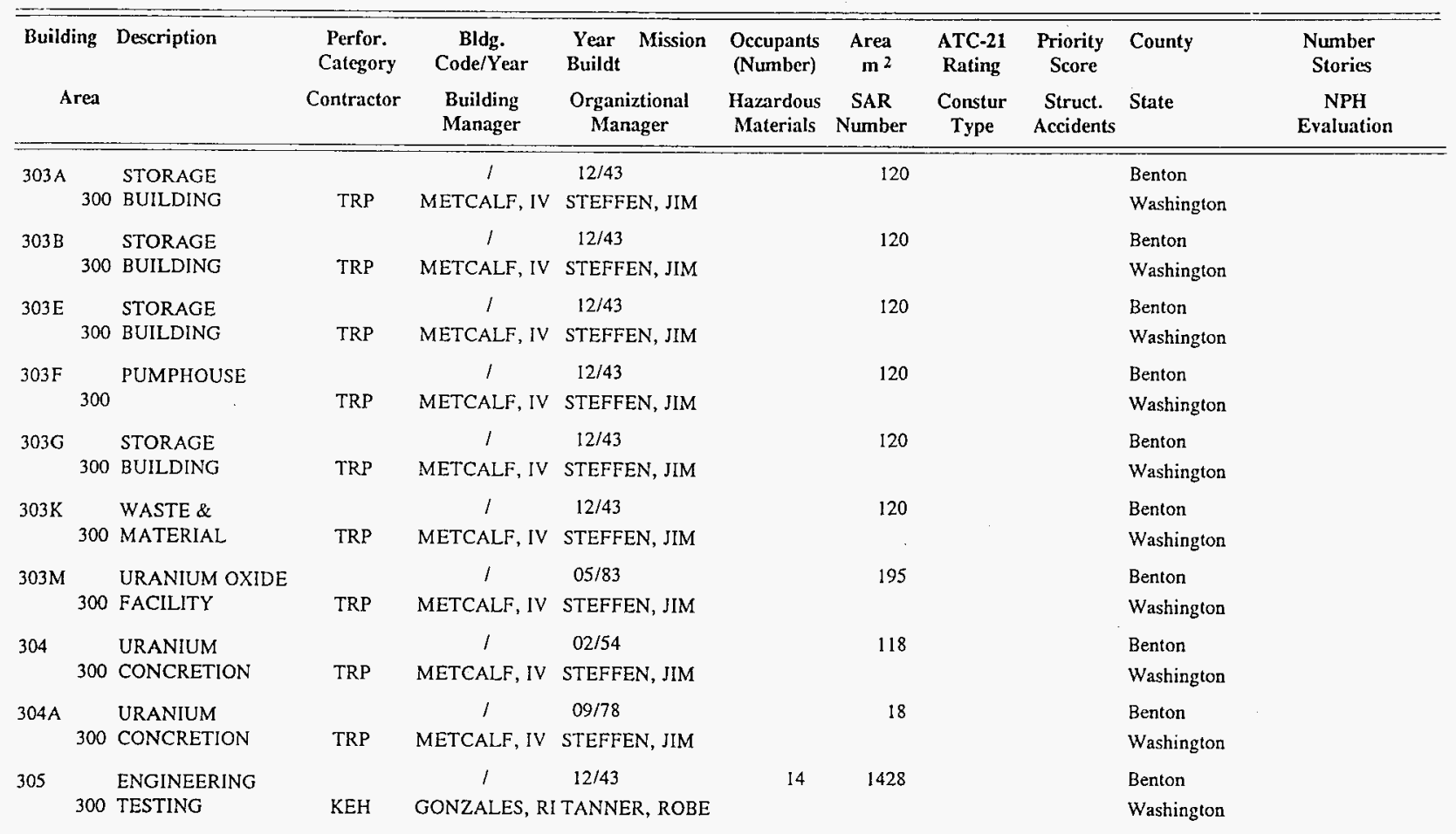


WHC-SD-SQA-CSWD-501 Rev 0

\begin{tabular}{|c|c|c|c|c|c|c|c|c|c|c|}
\hline Building & Description & $\begin{array}{c}\text { Perfor. } \\
\text { Category } \\
\text { Contractor }\end{array}$ & $\begin{array}{l}\text { Bldg. } \\
\text { Code/Year } \\
\text { Building } \\
\text { Manager }\end{array}$ & $\begin{array}{l}\text { Year Mission } \\
\text { Buildt } \\
\text { Organiztional } \\
\text { Manager }\end{array}$ & $\begin{array}{c}\text { Occupants } \\
\text { (Number) } \\
\text { Hazardous } \\
\text { Materials }\end{array}$ & $\begin{array}{c}\text { Area } \\
\mathrm{m}^{2} \\
\text { SAR } \\
\text { Number }\end{array}$ & $\begin{array}{c}\text { ATC-21 } \\
\text { Rating } \\
\text { Constur } \\
\text { Type }\end{array}$ & $\begin{array}{l}\text { Priority } \\
\text { Score } \\
\text { Struct. } \\
\text { Accidents }\end{array}$ & State & $\begin{array}{c}\text { Number } \\
\text { Stories } \\
\text { NPH } \\
\text { Evaluation }\end{array}$ \\
\hline $\begin{array}{ll}305 \mathrm{~A} & \\
& \\
& \end{array}$ & $\begin{array}{l}\text { ELECTRICIAN \& } \\
\text { PIPEFITTER SHOP }\end{array}$ & $\mathrm{KEH}$ & $\stackrel{\prime}{\text { RITTENHOUSE }}$ & RITTENHOUSE, & & 274 & & & $\begin{array}{l}\text { Benton } \\
\text { Washington }\end{array}$ & \\
\hline $\begin{array}{ll}305 P & \\
& 300\end{array}$ & $\begin{array}{l}\text { ENGINEERING } \\
\text { TESTING }\end{array}$ & STS & 1 & BROWN, LARRY & & 71 & & & $\begin{array}{l}\text { Benton } \\
\text { Washington }\end{array}$ & \\
\hline $\begin{array}{r}\text { 305WELL } \\
\text { RCHC }\end{array}$ & $\begin{array}{l}\text { LEASED } \\
\text { BUILDING }\end{array}$ & $\mathrm{KEH}$ & $\begin{array}{c}\prime \\
\text { TANNER RM }\end{array}$ & & & & & & $\begin{array}{l}\text { Benton } \\
\text { Washington }\end{array}$ & \\
\hline $306 \mathrm{E} 300$ & $\begin{array}{l}\text { DEVELOPMENT, } \\
\text { FABRICATION, \& }\end{array}$ & $\mathrm{KEH}$ & $\frac{1}{\text { PANTHER, DO }}$ & $\begin{array}{l}01 / 60 \\
\text { BROWN, LARRY }\end{array}$ & so & 3826 & & & $\begin{array}{l}\text { Benton } \\
\text { Washington }\end{array}$ & \\
\hline $\begin{array}{r}\text { 307WELL } \\
\text { RCHC }\end{array}$ & $\begin{array}{l}\text { LEASED } \\
\text { BUILDING }\end{array}$ & $\mathrm{KEH}$ & $\begin{array}{c}\text { / } \\
\text { TANNER RM }\end{array}$ & & & & & & $\begin{array}{l}\text { Benton } \\
\text { Washington }\end{array}$ & \\
\hline 300 & $\begin{array}{l}\text { FUELS } \\
\text { DEVELOPMENT }\end{array}$ & TRP & $\begin{array}{c}\prime \\
\text { BITTEN, ERNE }\end{array}$ & $\begin{array}{l}\text { 01/60 } \\
\text { STEFFEN, JIM }\end{array}$ & & 6609 & & & $\begin{array}{l}\text { Benton } \\
\text { Washington }\end{array}$ & \\
\hline $\begin{array}{l}308 \mathrm{~A} \\
\\
\end{array}$ & $\begin{array}{l}\text { FUELS } \\
\text { DEVELOPMENT }\end{array}$ & TRP & $\begin{array}{c}\text { I } \\
\text { BITTEN, ERNE }\end{array}$ & $\begin{array}{l}10 / 76 \\
\text { STEFFEN, JIM }\end{array}$ & & & & & $\begin{array}{l}\text { Benton } \\
\text { Washington }\end{array}$ & \\
\hline 300 & $\begin{array}{l}\text { SP-100 GES TEST } \\
\text { FACILITY }\end{array}$ & TRP & $\begin{array}{c}\text { I } \\
\text { BITTEN, ERNE }\end{array}$ & $\begin{array}{l}09 / 60 \\
\text { STEFFEN, JIM }\end{array}$ & & 4341 & & & $\begin{array}{l}\text { Benton } \\
\text { Washington }\end{array}$ & \\
\hline 300 & $\begin{array}{l}\text { TREATED } \\
\text { EFFLUENT }\end{array}$ & PSS & $\begin{array}{c}/ \\
\text { ROBERTS, LEE }\end{array}$ & $\begin{array}{l}07 / 94 \\
\text { DILIBERTO, AN }\end{array}$ & 9 & 1255 & & & $\begin{array}{l}\text { Benton } \\
\text { Washington }\end{array}$ & \\
\hline $\begin{array}{l}3100 \mathrm{GW} \\
\text { RCHN }\end{array}$ & $\begin{array}{l}\text { BUILDING - } \\
\text { LEASED }\end{array}$ & TRP & $\begin{array}{c}/ \\
\text { MCGINNIS KA }\end{array}$ & & 17 & & & & $\begin{array}{l}\text { Benton } \\
\text { Washington }\end{array}$ & \\
\hline
\end{tabular}


WHC-SD-SQA-CSWD-501 Rev 0

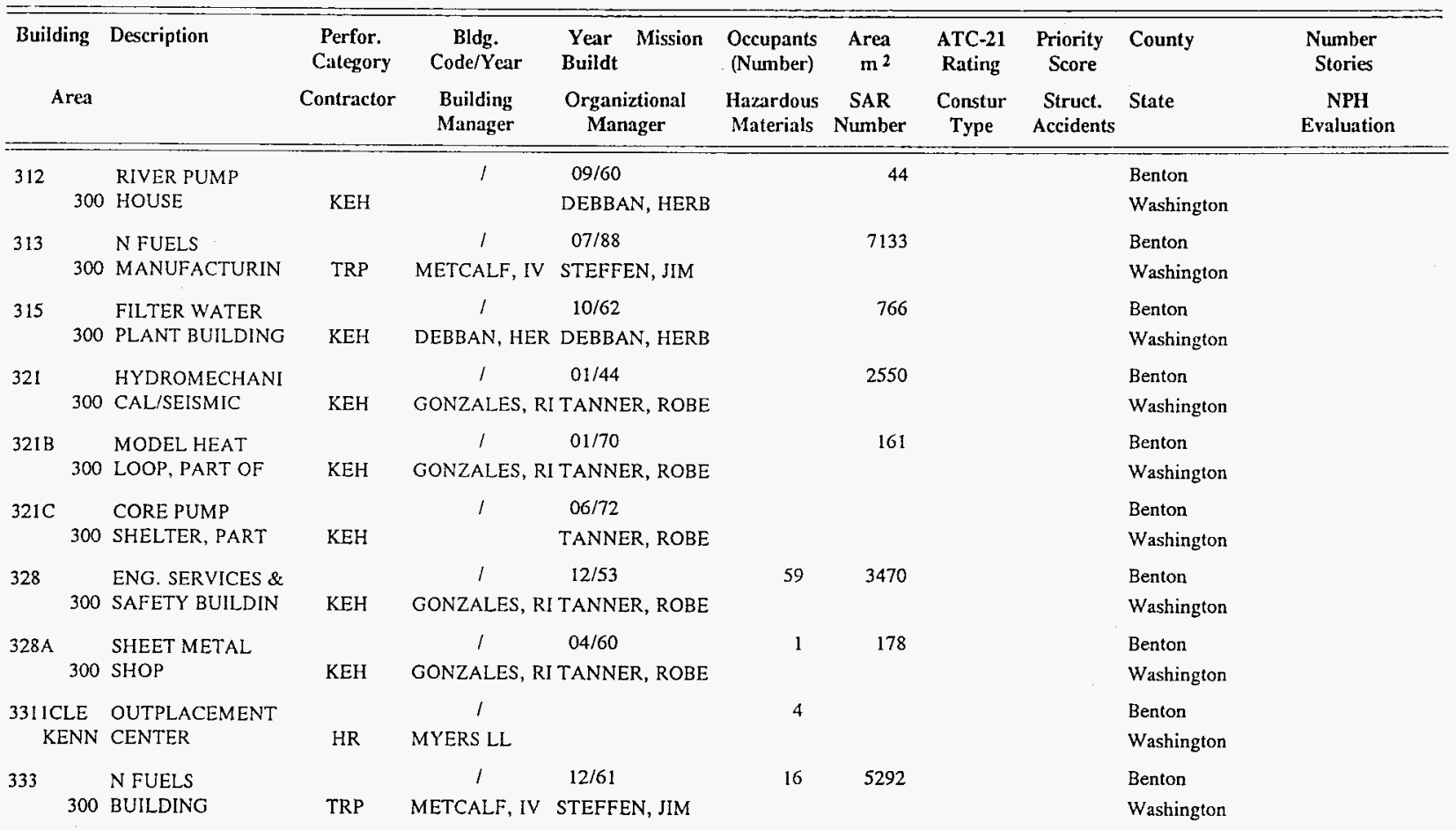


WHC-SD-SQA-CSWD-501 Rev 0

\begin{tabular}{|c|c|c|c|c|c|c|c|c|c|c|}
\hline Building & Description & $\begin{array}{l}\text { Perfor. } \\
\text { Category }\end{array}$ & $\begin{array}{c}\text { BIdg. } \\
\text { Code/Year }\end{array}$ & $\begin{array}{l}\text { Year Mission } \\
\text { Buildt }\end{array}$ & $\begin{array}{l}\text { Occupants } \\
\text { (Number) }\end{array}$ & $\begin{array}{r}\text { Area } \\
\mathrm{m}^{2}\end{array}$ & $\begin{array}{l}\text { ATC-21 } \\
\text { Rating }\end{array}$ & $\begin{array}{l}\text { Priority } \\
\text { Score }\end{array}$ & County & $\begin{array}{l}\text { Number } \\
\text { Stories }\end{array}$ \\
\hline Area & & Contractor & $\begin{array}{l}\text { Building } \\
\text { Manager }\end{array}$ & $\begin{array}{l}\text { Organiztional } \\
\text { Manager }\end{array}$ & $\begin{array}{l}\text { Hazardous } \\
\text { Materials }\end{array}$ & $\begin{array}{c}\text { SAR } \\
\text { Number }\end{array}$ & $\begin{array}{l}\text { Constur } \\
\text { Type }\end{array}$ & $\begin{array}{l}\text { Struct. } \\
\text { Accidents }\end{array}$ & State & $\begin{array}{c}\mathrm{NPH} \\
\text { Evaluation }\end{array}$ \\
\hline 300 & $\begin{array}{l}\text { PROCESS SEWER } \\
\text { MONITOR }\end{array}$ & TRP & $\begin{array}{c}I \\
\text { METCALF, IV }\end{array}$ & $\begin{array}{l}12 / 61 \\
\text { STEFFEN, JIM }\end{array}$ & & 28 & & & $\begin{array}{l}\text { Benton } \\
\text { Washington }\end{array}$ & \\
\hline $\begin{array}{l}334 \mathrm{~A} \\
\end{array}$ & $\begin{array}{l}\text { WASTE ACID } \\
\text { STORAGE }\end{array}$ & TRP & $\begin{array}{c}/ \\
\text { METCALF, IV }\end{array}$ & $\begin{array}{l}\text { 04/59 } \\
\text { STEFFEN, JIM }\end{array}$ & & 74 & & & $\begin{array}{l}\text { Benton } \\
\text { Washington }\end{array}$ & \\
\hline 300 & $\begin{array}{l}\text { SODIUM TEST } \\
\text { FACILITY }\end{array}$ & TRP & $\begin{array}{c}\prime \\
\text { HUNTER, JAM }\end{array}$ & $\begin{array}{l}11 / 69 \\
\text { LOIKA, EDWAR }\end{array}$ & & 620 & & & $\begin{array}{l}\text { Benton } \\
\text { Washington }\end{array}$ & \\
\hline $337 \mathrm{~B}$ & $\begin{array}{l}337 \text { HIGH-BAY } \\
\text { AND SERVICE }\end{array}$ & KEH & $\begin{array}{c}/ \\
\text { GONZALES, RI }\end{array}$ & $\begin{array}{l}05 / 73 \\
\text { I TANNER, ROBE }\end{array}$ & & 3446 & & & $\begin{array}{l}\text { Benton } \\
\text { Washington }\end{array}$ & \\
\hline $339 \mathrm{~A}$ & $\begin{array}{l}\text { COMPUTER } \\
\text { FACILITY }\end{array}$ & $\mathrm{BCS}$ & $\begin{array}{c}\prime \\
\text { ELISON, BARY }\end{array}$ & $\begin{array}{l}\text { 09/88 } \\
\text { ESTEY, SANDR }\end{array}$ & 17 & 446 & & & $\begin{array}{l}\text { Benton } \\
\text { Washington }\end{array}$ & \\
\hline 300 & $\begin{array}{l}\text { WASTE } \\
\text { NEUTRALIZATIO }\end{array}$ & PSS & $\begin{array}{c}\prime \\
\text { ROBERTS, LEE }\end{array}$ & $\begin{array}{l}12 / 53 \\
\text { DILIBERTO, AN }\end{array}$ & & 416 & & & $\begin{array}{l}\text { Benton } \\
\text { Washington }\end{array}$ & \\
\hline $340 \mathrm{~A}$ & $\begin{array}{l}\text { WASTE } \\
\text { RETENTION }\end{array}$ & PSS & $\begin{array}{c}\prime \\
\text { ROBERTS, LEE }\end{array}$ & $\begin{array}{l}01 / 61 \\
\text { DILIBERTO, AN }\end{array}$ & & 127 & & & $\begin{array}{l}\text { Benton } \\
\text { Washington }\end{array}$ & \\
\hline 300 & $\begin{array}{l}\text { WASTE } \\
\text { LOADOUT }\end{array}$ & PSS & $\begin{array}{c}\prime \\
\text { ROBERTS, LEE }\end{array}$ & $\begin{array}{l}03 / 69 \\
\text { DILIBERTO, AN }\end{array}$ & & 112 & & & $\begin{array}{l}\text { Benton } \\
\text { Washington }\end{array}$ & \\
\hline $\begin{array}{l}342 A \\
\end{array} 300$ & $\begin{array}{l}\text { INSTR/ELEC } \\
\text { BUILDING SHOP }\end{array}$ & PSS & 1 & $\begin{array}{l}\text { 07/94 } \\
\text { DILIBERTO, AN }\end{array}$ & & 16 & & & $\begin{array}{l}\text { Benton } \\
\text { Washington }\end{array}$ & \\
\hline $\begin{array}{l}3506 \mathrm{~A} \\
300\end{array}$ & $\begin{array}{l}\text { POWERHOUSE } \\
\text { MAINTENANCE }\end{array}$ & KEH & $\begin{array}{c}/ \\
\text { GONZALES, RI }\end{array}$ & TANNER, ROBE & 3 & 175 & & & $\begin{array}{l}\text { Benton } \\
\text { Washington }\end{array}$ & \\
\hline
\end{tabular}


WHC-SD-SQA-CSWD-501 Rev 0

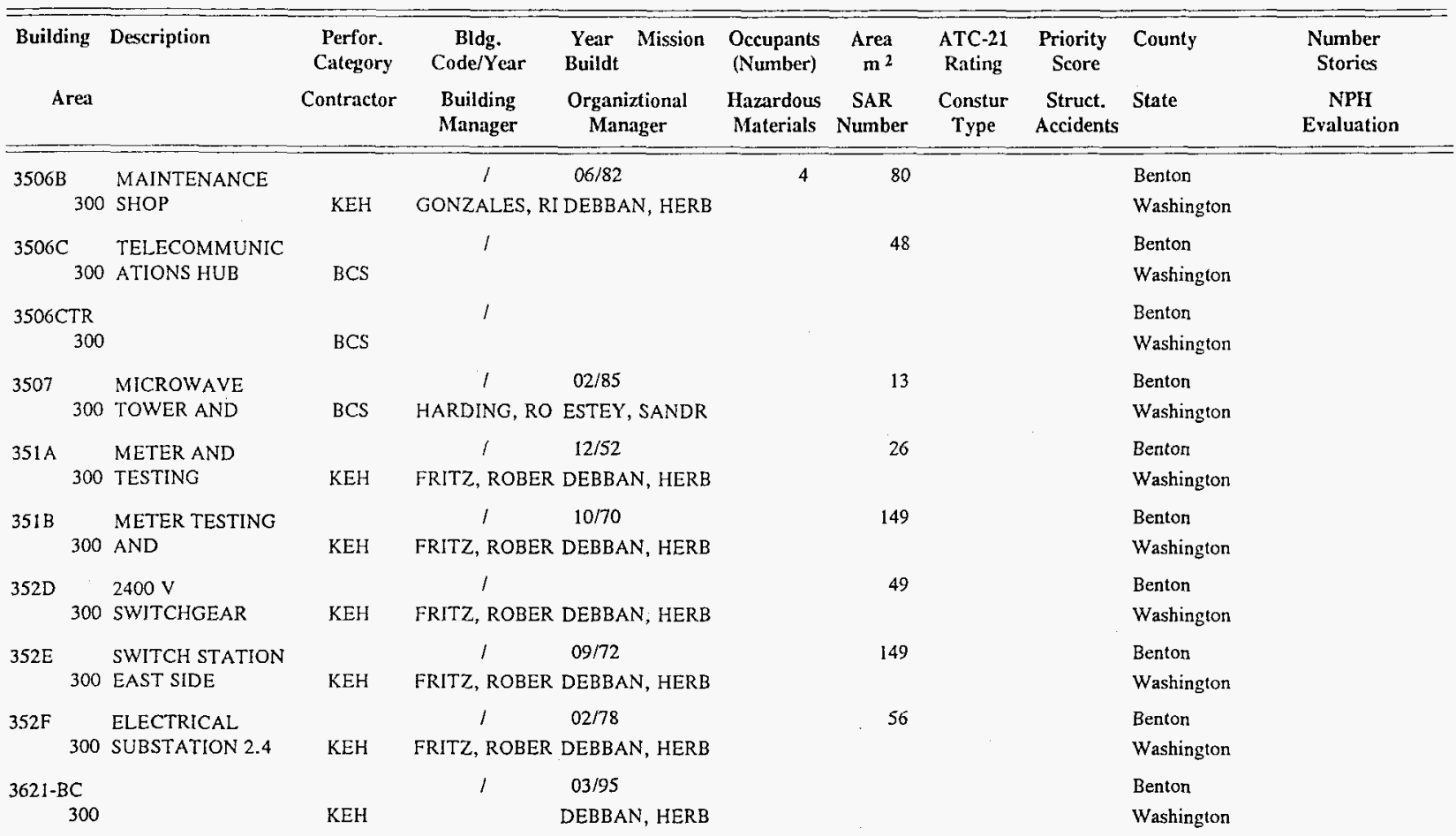


WHC-SD-SQA-CSWD-501 Rev 0

\begin{tabular}{|c|c|c|c|c|c|c|c|c|c|c|}
\hline Building & Description & $\begin{array}{l}\text { Perfor. } \\
\text { Category } \\
\text { Contractor }\end{array}$ & $\begin{array}{c}\text { Bldg. } \\
\text { Code/Year } \\
\text { Building } \\
\text { Manager }\end{array}$ & $\begin{array}{l}\text { Year Mission } \\
\text { Buildt } \\
\text { Organiztional } \\
\text { Manager }\end{array}$ & $\begin{array}{l}\text { Occupants } \\
\text { (Number) } \\
\text { Hazardous } \\
\text { Materials }\end{array}$ & $\begin{array}{c}\text { Area } \\
\mathrm{m}^{2} \\
\text { SAR } \\
\text { Number }\end{array}$ & $\begin{array}{c}\text { ATC-21 } \\
\text { Rating } \\
\text { Constur } \\
\text { Type }\end{array}$ & $\begin{array}{l}\text { Priority } \\
\text { Score } \\
\text { Struct. } \\
\text { Accidents }\end{array}$ & State & $\begin{array}{c}\text { Number } \\
\text { Stories } \\
\text { NPH } \\
\text { Evaluation }\end{array}$ \\
\hline $\begin{array}{r}3621 \mathrm{D} \\
300\end{array}$ & $\begin{array}{l}\text { EMERGENCY } \\
\text { GENERATOR }\end{array}$ & $\mathrm{KEH}$ & $\begin{array}{c}/ \\
\text { DEBBAN, HER }\end{array}$ & $\begin{array}{l}05 / 74 \\
\text { DEBBAN, HERB }\end{array}$ & & 238 & & & $\begin{array}{l}\text { Benton } \\
\text { Washington }\end{array}$ & \\
\hline $\begin{array}{r}3701 \mathrm{D} \\
300\end{array}$ & $\begin{array}{l}\text { BENTON CO. } \\
\text { SHERIFF }\end{array}$ & $\mathrm{KEH}$ & $\begin{array}{c}\prime \\
\text { GONZALES, RI }\end{array}$ & $\begin{array}{l}02 / 83 \\
\text { I TANNER, ROBE }\end{array}$ & 1 & 614 & & & $\begin{array}{l}\text { Benton } \\
\text { Washington }\end{array}$ & \\
\hline $\begin{array}{r}3701 \mathrm{U} \\
300\end{array}$ & OFFICE BUILDING & $\mathrm{KEH}$ & $\begin{array}{c}\prime \\
\text { GONZALES, RI }\end{array}$ & $\begin{array}{l}\text { 07/80 } \\
\text { I TANNER, ROBE }\end{array}$ & & 125 & & & $\begin{array}{l}\text { Benton } \\
\text { Washington }\end{array}$ & \\
\hline $\begin{array}{r}3703 \mathrm{~A} \\
300\end{array}$ & $\begin{array}{l}\text { MODULAR } \\
\text { OFFICES }\end{array}$ & $\mathrm{KEH}$ & 1 & $\begin{array}{l}03 / 96 \\
\text { RITTENHOUSE, }\end{array}$ & 7 & 172 & & & $\begin{array}{l}\text { Benton } \\
\text { Washington }\end{array}$ & . \\
\hline 300 & $\begin{array}{l}\text { INSULATORS } \\
\text { STORAGE }\end{array}$ & $\mathrm{KEH}$ & $\begin{array}{c}\prime \\
\text { HOLLADAY, J }\end{array}$ & $\begin{array}{l}\text { 09/42 } \\
\text { RITTENHOUSE, }\end{array}$ & & 74 & & & $\begin{array}{l}\text { Benton } \\
\text { Washington }\end{array}$ & \\
\hline 300 & $\begin{array}{l}\text { PHOTOGRAPHY } \\
\text { BUILDING }\end{array}$ & $\mathrm{KEH}$ & $\begin{array}{c}/ \\
\text { GONZALES, RI }\end{array}$ & I TANNER, ROBE & 10 & 648 & & & $\begin{array}{l}\text { Benton } \\
\text { Washington }\end{array}$ & \\
\hline 300 & $\begin{array}{l}\text { COMMUN. \& } \\
\text { DOCUMENTATIO }\end{array}$ & $\mathrm{KEH}$ & $\begin{array}{c}\text { I } \\
\text { GONZALES, RI }\end{array}$ & $\begin{array}{l}12 / 44 \\
\text { I TANNER, ROBE }\end{array}$ & & 3482 & & & $\begin{array}{l}\text { Benton } \\
\text { Washington }\end{array}$ & \\
\hline $\begin{array}{r}3706 \mathrm{~A} \\
300\end{array}$ & $\begin{array}{l}\text { VENTILATION } \\
\text { EQUIP RM FOR }\end{array}$ & KEH & $\begin{array}{c}l \\
\text { GONZALES, RI }\end{array}$ & $\begin{array}{l}12 / 44 \\
\text { I TANNER, ROBE }\end{array}$ & & 140 & & & $\begin{array}{l}\text { Benton } \\
\text { Washington }\end{array}$ & \\
\hline $\begin{array}{r}3707 \mathrm{D} \\
300\end{array}$ & $\begin{array}{l}\text { INFORMATION } \\
\text { SERVICES }\end{array}$ & $\mathrm{KEH}$ & $\begin{array}{c}/ \\
\text { GONZALES, RI }\end{array}$ & $\begin{array}{l}\text { 06/55 } \\
\text { TANNER, ROBE }\end{array}$ & 15 & 797 & & & $\begin{array}{l}\text { Benton } \\
\text { Washington }\end{array}$ & \\
\hline $3707 \mathrm{E}_{300}$ & $\begin{array}{l}\text { CONSTRUCTION } \\
\text { STORAGE }\end{array}$ & KEH & $\begin{array}{c}\text { / } \\
\text { DEBBAN, HER }\end{array}$ & $\begin{array}{l}09 / 42 \\
\text { RITTENHOUSE, }\end{array}$ & & 74 & & & $\begin{array}{l}\text { Benton } \\
\text { Washington }\end{array}$ & \\
\hline
\end{tabular}


WHC-SD-SQA-CSWD-501 Rev 0

\begin{tabular}{|c|c|c|c|c|c|c|c|c|c|c|}
\hline Building & Description & $\begin{array}{l}\text { Perfor. } \\
\text { Category }\end{array}$ & $\begin{array}{l}\text { Bldg. } \\
\text { Code/Year }\end{array}$ & $\begin{array}{l}\text { Year Mission } \\
\text { Buildt }\end{array}$ & $\begin{array}{l}\text { Occupants } \\
\text { (Number) }\end{array}$ & $\begin{array}{r}\text { Area } \\
\mathrm{m}^{2}\end{array}$ & $\begin{array}{l}\text { ATC-21 } \\
\text { Rating }\end{array}$ & $\begin{array}{l}\text { Priority } \\
\text { Score }\end{array}$ & County & $\begin{array}{l}\text { Number } \\
\text { Stories }\end{array}$ \\
\hline Area & & Contractor & $\begin{array}{l}\text { Building } \\
\text { Manager }\end{array}$ & $\begin{array}{l}\text { Organiztional } \\
\text { Manager }\end{array}$ & $\begin{array}{l}\text { Hazardous } \\
\text { Materials }\end{array}$ & $\begin{array}{l}\text { SAR } \\
\text { Number }\end{array}$ & $\begin{array}{c}\text { Constur } \\
\text { Type }\end{array}$ & $\begin{array}{l}\text { Struct. } \\
\text { Accidents }\end{array}$ & State & $\begin{array}{c}\text { NPH } \\
\text { Evaluation }\end{array}$ \\
\hline $\begin{array}{l}3707 \mathrm{EA} \\
300\end{array}$ & PAINT STORAGE & KEH & 1 & & & 26 & & & $\begin{array}{l}\text { Benton } \\
\text { Washington }\end{array}$ & \\
\hline $\begin{array}{r}3707 \mathrm{~F} \\
300\end{array}$ & $\begin{array}{l}\text { RADIATION } \\
\text { MONITORING }\end{array}$ & PSS & $\begin{array}{c}\ell \\
\text { GONZALES, } \mathrm{R}\end{array}$ & $\begin{array}{l}02 / 65 \\
\text { DILIBERTO, AN }\end{array}$ & & 13 & & & $\begin{array}{l}\text { Benton } \\
\text { Washington }\end{array}$ & \\
\hline $\begin{array}{l}3707 \mathrm{H} \\
300\end{array}$ & CHANGE HOUSE & KEH & $\begin{array}{c}\prime \\
\text { GONZALES, R }\end{array}$ & ITANNER, ROBE & & 112 & & & $\begin{array}{l}\text { Benton } \\
\text { Washington }\end{array}$ & \\
\hline 3709 & PAINT SHOP & $\mathrm{KEH}$ & $\begin{array}{c}/ \\
\text { GONZALES, R }\end{array}$ & $\begin{array}{l}12 / 43 \\
\text { TANNER, ROBE }\end{array}$ & 2 & 289 & & & $\begin{array}{l}\text { Benton } \\
\text { Washington }\end{array}$ & \\
\hline $\begin{array}{r}3709 \mathrm{~A} \\
300\end{array}$ & FIRE STATION & ESQ & $\begin{array}{c}I \\
\text { GOOD, DONA }\end{array}$ & $\begin{array}{l}08 / 64 \\
\text { GOOD, DONAL }\end{array}$ & 31 & 776 & & & $\begin{array}{l}\text { Benton } \\
\text { Washington }\end{array}$ & \\
\hline $\begin{array}{l}3709 \mathrm{~B} \\
300\end{array}$ & $\begin{array}{l}\text { FIRE EQUIPMENT } \\
\text { STORAGE }\end{array}$ & ESQ & GOOD, DONA & $\begin{array}{l}02 / 77 \\
\text { GOOD, DONAL }\end{array}$ & & 56 & & & $\begin{array}{l}\text { Benton } \\
\text { Washington }\end{array}$ & \\
\hline $\begin{array}{l}3710 \mathrm{~A} \\
300\end{array}$ & $\begin{array}{l}\text { OIL STORAGE } \\
\text { BUILDING }\end{array}$ & KEH & $\begin{array}{c}/ \\
\text { GONZALES, } R\end{array}$ & $\begin{array}{l}\text { 11/61 } \\
\text { TANNER, ROBE }\end{array}$ & & 25 & & & $\begin{array}{l}\text { Benton } \\
\text { Washington }\end{array}$ & \\
\hline 300 & $\begin{array}{l}\text { MAINTENANCE } \\
\text { STORAGE }\end{array}$ & KEH & $\begin{array}{c}/ \\
\text { GONZALES, R }\end{array}$ & $\begin{array}{l}11 / 59 \\
\text { TANNER, ROBE }\end{array}$ & & 297 & & & $\begin{array}{l}\text { Benton } \\
\text { Washington }\end{array}$ & \\
\hline 300 & $\begin{array}{l}\text { STORAGE } \\
\text { BUILDING }\end{array}$ & TRP & $\begin{array}{c}/ \\
\text { METCALF, IV }\end{array}$ & $\begin{array}{l}08 / 67 \\
\text { STEFFEN, JIM }\end{array}$ & & 903 & & & $\begin{array}{l}\text { Benton } \\
\text { Washington }\end{array}$ & \\
\hline 300 & $\begin{array}{l}\text { CARPENTER } \\
\text { SHOP }\end{array}$ & KEH & $\begin{array}{c}/ \\
\text { GONZALES, R }\end{array}$ & $\begin{array}{l}12 / 43 \\
\text { TANNER, ROBE }\end{array}$ & 7 & 446 & & & $\begin{array}{l}\text { Benton } \\
\text { Washington }\end{array}$ & \\
\hline
\end{tabular}


WHC-SD-SQA-CSWD-501 Rev 0

\begin{tabular}{|c|c|c|c|c|c|c|c|c|c|c|}
\hline \multirow{2}{*}{$\begin{array}{l}\text { Building } \\
\text { Area }\end{array}$} & \multirow[t]{2}{*}{ Description } & \multirow{2}{*}{$\begin{array}{l}\text { Perfor. } \\
\text { Category } \\
\text { Contractor }\end{array}$} & \multirow{2}{*}{$\begin{array}{c}\text { Bldg. } \\
\text { Code/Year } \\
\text { Building } \\
\text { Manager }\end{array}$} & \multirow{2}{*}{$\begin{array}{l}\text { Year Mission } \\
\text { Buildt } \\
\text { Organiztional } \\
\text { Manager }\end{array}$} & \multirow{2}{*}{$\begin{array}{c}\text { Occupants } \\
\text { (Number) } \\
\text { Hazardous } \\
\text { Materials }\end{array}$} & \multirow{2}{*}{$\begin{array}{c}\text { Area } \\
\mathrm{m}^{2} \\
\text { SAR } \\
\text { Number }\end{array}$} & \multirow{2}{*}{$\begin{array}{c}\text { ATC-21 } \\
\text { Rating } \\
\text { Constur } \\
\text { Type }\end{array}$} & \multirow{2}{*}{$\begin{array}{l}\text { Priority } \\
\text { Score } \\
\text { Struct. } \\
\text { Accidents }\end{array}$} & \multirow{2}{*}{$\begin{array}{l}\text { County } \\
\text { State }\end{array}$} & \multirow{2}{*}{$\begin{array}{c}\text { Number } \\
\text { Stories } \\
\text { NPH } \\
\text { Evaluation }\end{array}$} \\
\hline & & & & & & & & & & \\
\hline 3714 & SOILS & & 1 & & & 107 & & & Benton & \\
\hline 300 & LABORATORY & PSS & DILIBERTO, A & DILIBERTO, AN & & & & & Washinglon & \\
\hline 3715 & STORAGE & & 1 & $02 / 59$ & & 595 & & & Benton & \\
\hline 300 & BUILDING & $\mathrm{KEH}$ & GONZALES, R & I TANNER, ROBE & & & & & Washington & \\
\hline 3716 & STORAGE & & 1 & $01 / 38$ & & 297 & & & Benton & \\
\hline 300 & BUILDING & TRP & METCALF, IV & STEFFEN, JIM & & & & & Washington & \\
\hline 3717 & SPARE PARTS & & 1 & $12 / 44$ & & 897 & & & Benton & \\
\hline 300 & WAREHOUSE & $\mathrm{KEH}$ & GONZALES, R & I TANNER, ROBE & & & & & Washington & \\
\hline $3717 \mathrm{~B}$ & STANDARDS & & 1 & $08 / 94$ & 12 & 827 & & & Benton & \\
\hline 300 & LABORATORY & $\mathrm{KEH}$ & GONZALES, R & I TANNER, ROBE & & & & & Washington & \\
\hline $3717 \mathrm{C}$ & MATERIALS & & 1 & & & 214 & & & Benton & \\
\hline 300 & ARCHIVE & KEH & GONZALES, $\mathrm{R}$ & I TANNER, ROBE & & & & & Washington & \\
\hline 3718 & OFFICE \& & & 1 & $11 / 59$ & 10 & 355 & & & Benton & \\
\hline 300 & STORAGE & KEH & GONZALES, $\mathrm{R}$ & I TANNER, ROBE & & & & & Washington & \\
\hline $3718 \mathrm{C}$ & STORAGE & & 1 & & & 416 & & & Benton & \\
\hline 300 & BUILDING & KEH & GONZALES, R & I RITTENHOUSE, & & & & & Washington & \\
\hline $3718 \mathrm{~F}$ & SODIUM & & 1 & $09 / 46$ & & 89 & & & Benton & \\
\hline 300 & STORAGE & PSS & GONZALES, R & I DILIBERTO, AN & & & & & Washington & \\
\hline $3718 \mathrm{M}$ & SODIUM & & 1 & $05 / 73$ & & 187 & & & Benton & \\
\hline 300 & STORAGE & KEH & GONZALES, R & TANNER, ROBE & & & & & Washington & \\
\hline
\end{tabular}


WHC-SD-SQA-CSWD-501 Rev 0

\begin{tabular}{|c|c|c|c|c|c|c|c|c|c|c|}
\hline Building & Description & $\begin{array}{l}\text { Perfor. } \\
\text { Category }\end{array}$ & $\begin{array}{l}\text { Bldg. } \\
\text { Code/Year }\end{array}$ & $\begin{array}{l}\text { Year Mission } \\
\text { Buildt }\end{array}$ & $\begin{array}{l}\text { Occupants } \\
\text { (Number) }\end{array}$ & $\begin{array}{r}\text { Area } \\
\mathbf{m}^{2}\end{array}$ & $\begin{array}{l}\text { ATC-21 } \\
\text { Rating }\end{array}$ & $\begin{array}{c}\text { Priority } \\
\text { Score }\end{array}$ & County & $\begin{array}{l}\text { Number } \\
\text { Stories }\end{array}$ \\
\hline Area & & Contractor & $\begin{array}{l}\text { Building } \\
\text { Manager }\end{array}$ & $\begin{array}{c}\text { Organiztional } \\
\text { Manager }\end{array}$ & $\begin{array}{l}\text { Hazardous } \\
\text { Materials }\end{array}$ & $\begin{array}{c}\text { SAR } \\
\text { Number }\end{array}$ & $\begin{array}{l}\text { Constur } \\
\text { Type }\end{array}$ & $\begin{array}{l}\text { Struct. } \\
\text { Accidents }\end{array}$ & State & $\begin{array}{c}\text { NPH } \\
\text { Evaluation }\end{array}$ \\
\hline $3718 \mathrm{~N}$ & INSULATION & & 1 & $09 / 71$ & 1 & 268 & & & Benton & \\
\hline 300 & SHOP & $\mathrm{KEH}$ & GONZALES, & I TANNER, ROBE & & & & & Washington & \\
\hline $\begin{array}{r}37185 \\
300\end{array}$ & & KEH & 1 & & & & & & $\begin{array}{l}\text { Benton } \\
\text { Washington }\end{array}$ & \\
\hline 300 & $\begin{array}{l}\text { COMPUTER } \\
\text { FACILITY }\end{array}$ & $\mathrm{KEH}$ & $\begin{array}{c}l \\
\text { GONZALES, }\end{array}$ & $\begin{array}{l}02 / 79 \\
\text { TANNER, ROBE }\end{array}$ & 1 & 280 & & & $\begin{array}{l}\text { Benton } \\
\text { Washington }\end{array}$ & \\
\hline $\begin{array}{r}3719 \mathrm{~A} \\
300\end{array}$ & $\begin{array}{l}\text { MODULAR FIRST } \\
\text { AID STATION - TE }\end{array}$ & KEH & $\begin{array}{c}l \\
\text { GONZALES, } \mathrm{F}\end{array}$ & $\begin{array}{l}02 / 96 \\
\text { I TANNER, ROBE }\end{array}$ & & 172 & & & $\begin{array}{l}\text { Benton } \\
\text { Washington }\end{array}$ & \\
\hline $\begin{array}{ll}3721 & \\
& 300\end{array}$ & $\begin{array}{l}\text { CLASSIFIED } \\
\text { INCINERATOR }\end{array}$ & $\mathrm{KEH}$ & $\begin{array}{c}\prime \\
\text { HOWSER, VIR }\end{array}$ & TANNER, ROBE & & 22 & & & $\begin{array}{l}\text { Benton } \\
\text { Washington }\end{array}$ & \\
\hline $\begin{array}{l}3722 \\
\\
\end{array}$ & $\begin{array}{l}\text { FABRICATION } \\
\text { SHOP }\end{array}$ & $\mathrm{KEH}$ & $\begin{array}{c}l \\
\text { RITTENHOUS }\end{array}$ & RITTENHOUSE, & & 468 & & & $\begin{array}{l}\text { Benton } \\
\text { Washington }\end{array}$ & \\
\hline $\begin{array}{l}3727 \\
\\
\end{array}$ & $\begin{array}{l}\text { CLASSIFIED } \\
\text { VAULT }\end{array}$ & $\mathrm{KEH}$ & $\begin{array}{c}\text { I } \\
\text { GONZALES, } R\end{array}$ & $\begin{array}{l}07 / 77 \\
\text { TANNER, ROBE }\end{array}$ & & 81 & & & $\begin{array}{l}\text { Benton } \\
\text { Washington }\end{array}$ & \\
\hline $\begin{array}{ll}3728 & \\
& 300\end{array}$ & $\begin{array}{l}\text { GEOTECHNICAL. } \\
\text { HIGH-BAY }\end{array}$ & PSS & $\begin{array}{c}1 \\
\text { GONZALES, }\end{array}$ & $\begin{array}{l}07 / 81 \\
\text { DILIBERTO, AN }\end{array}$ & & 297 & & & $\begin{array}{l}\text { Benton } \\
\text { Washington }\end{array}$ & \\
\hline $\begin{array}{l}3732 \\
\\
\end{array}$ & $\begin{array}{l}\text { STORAGE } \\
\text { BUILDING }\end{array}$ & KEH & $\begin{array}{c}\prime \\
\text { GONZALES, R }\end{array}$ & $\begin{array}{l}09 / 49 \\
\text { TANNER, ROBE }\end{array}$ & & 127 & & & $\begin{array}{l}\text { Benton } \\
\text { Washington }\end{array}$ & \\
\hline 3734300 & $\begin{array}{l}\text { MAINTENANCE } \\
\text { STORAGE }\end{array}$ & $\mathrm{KEH}$ & $\begin{array}{c}\prime \\
\text { GONZALES, }\end{array}$ & $\begin{array}{l}12 / 44 \\
\text { TANNER, ROBE }\end{array}$ & & 22 & & & $\begin{array}{l}\text { Benton } \\
\text { Washington }\end{array}$ & \\
\hline
\end{tabular}


WHC-SD-SQA-CSWD-501 Rev 0

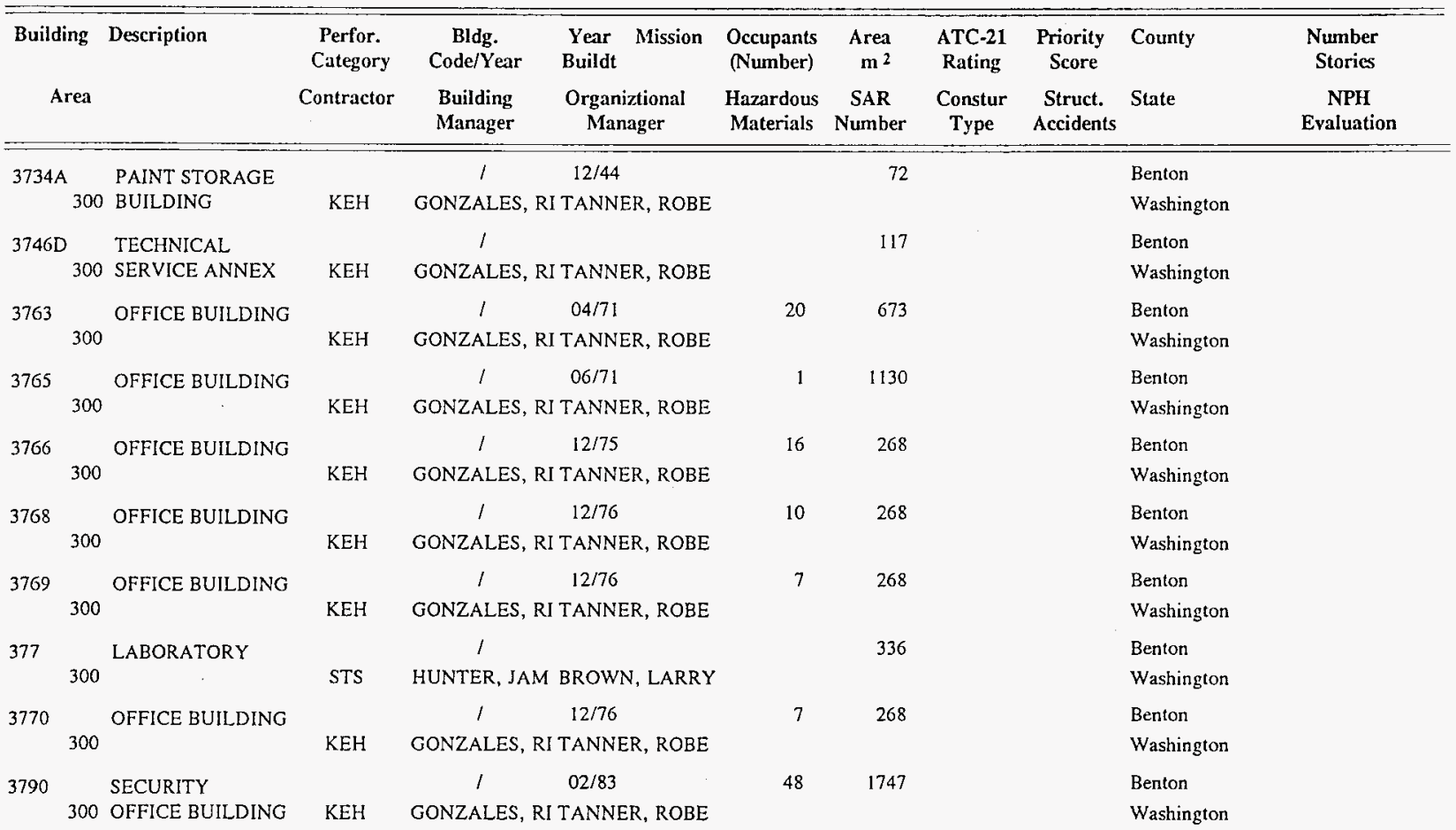


WHC-SD-SQA-CSWD-501 Rev 0

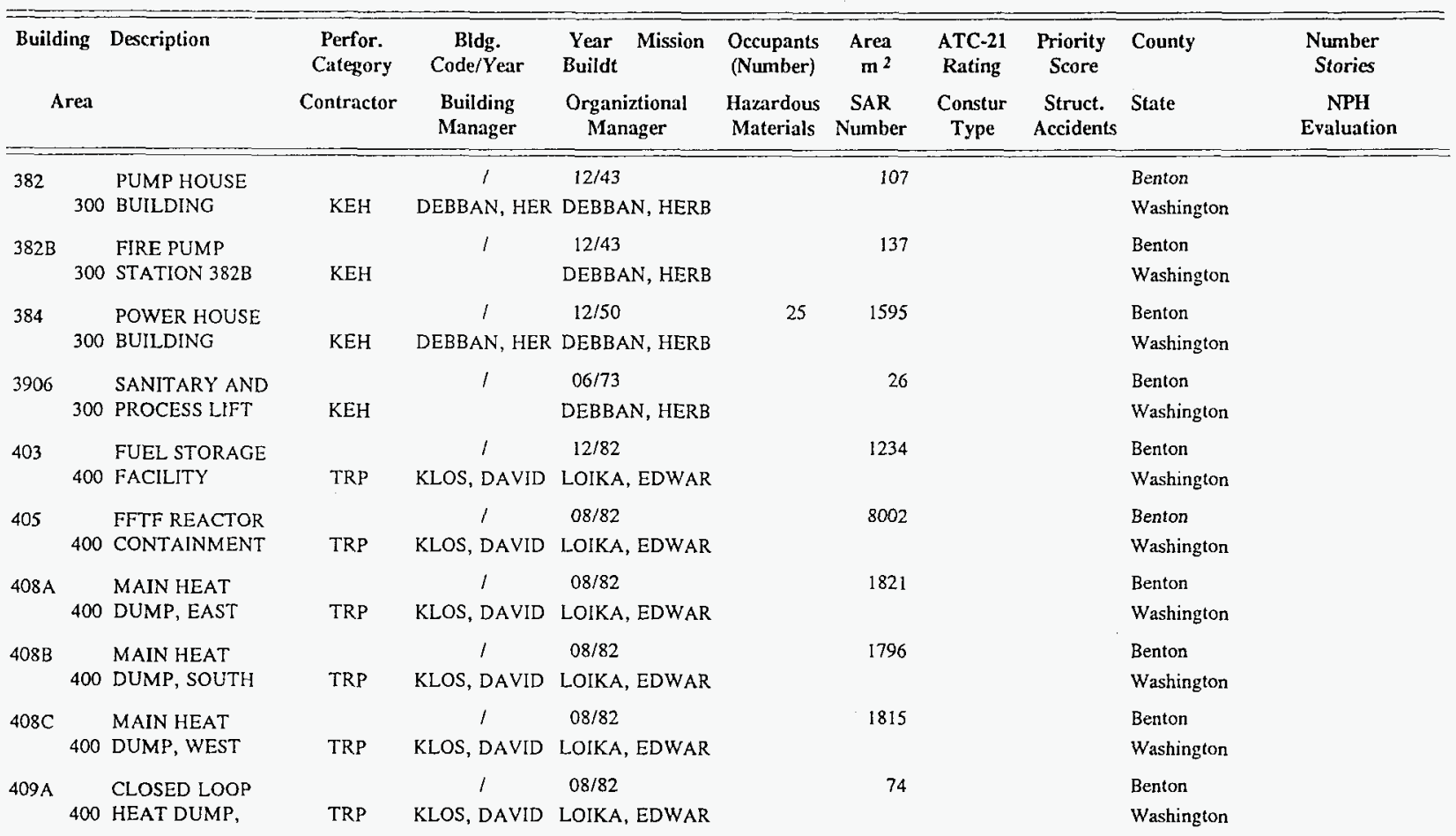


WHC-SD-SQA-CSWD-501 Rev 0

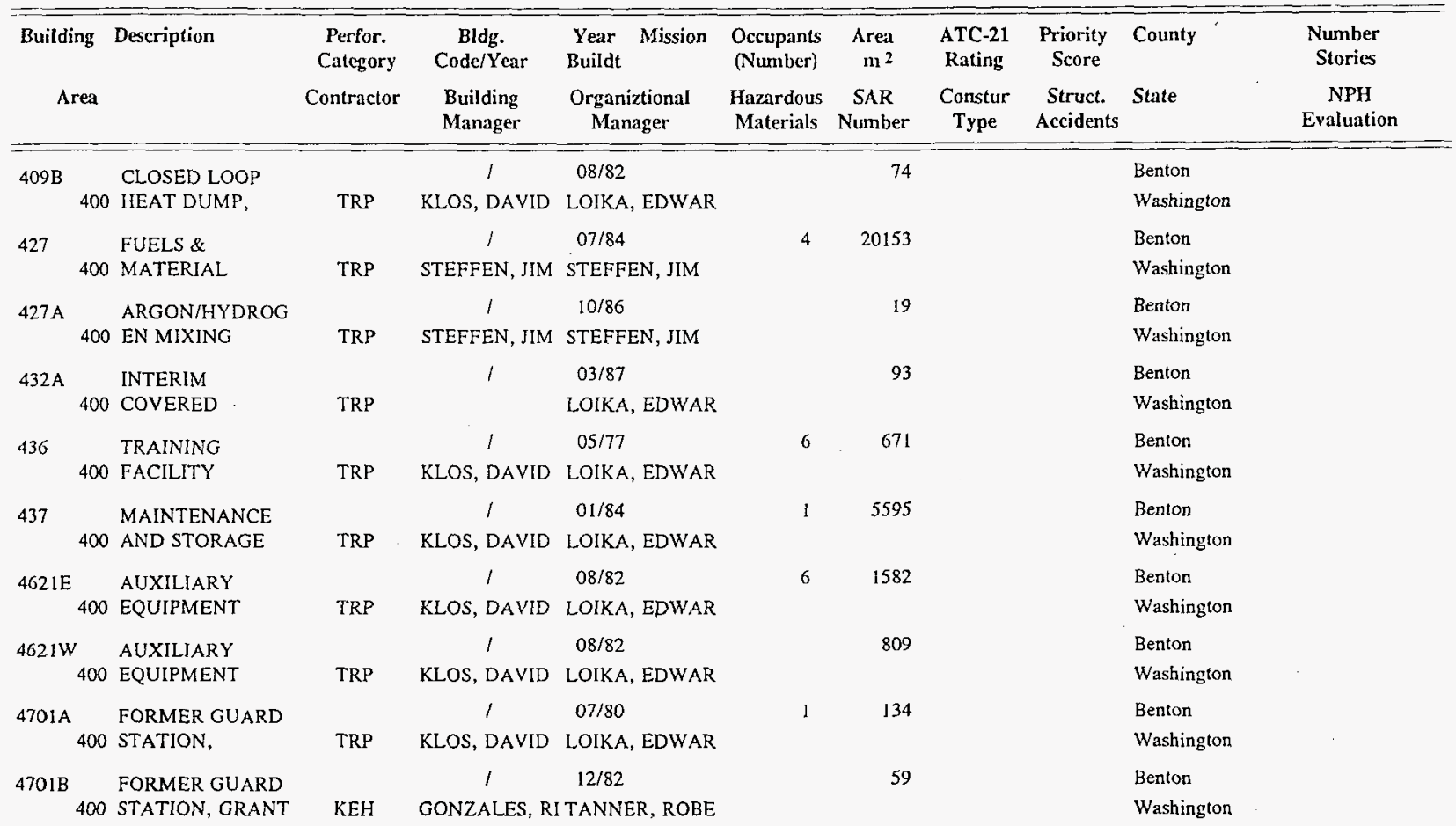


WHC-SD-SQA-CSWD-501 Rev 0

\begin{tabular}{|c|c|c|c|c|c|c|c|c|c|c|}
\hline Building & Description & $\begin{array}{c}\text { Perfor. } \\
\text { Category } \\
\text { Contractor }\end{array}$ & $\begin{array}{c}\text { Bldg. } \\
\text { Code/Year } \\
\text { Building } \\
\text { Manager }\end{array}$ & $\begin{array}{l}\text { Year Mission } \\
\text { Buildt } \\
\text { Organiztional } \\
\text { Manager }\end{array}$ & $\begin{array}{l}\text { Occupants } \\
\text { (Number) } \\
\text { Hazardous } \\
\text { Materials }\end{array}$ & $\begin{array}{c}\text { Area } \\
\text { m }^{2} \\
\text { SAR } \\
\text { Number }\end{array}$ & $\begin{array}{l}\text { ATC-21 } \\
\text { Rating } \\
\text { Constur } \\
\text { Type }\end{array}$ & $\begin{array}{l}\text { Priority } \\
\text { Score } \\
\text { Struct. } \\
\text { Accidents }\end{array}$ & State & $\begin{array}{c}\text { Number } \\
\text { Stories } \\
\text { NPH } \\
\text { Evaluation }\end{array}$ \\
\hline $4701 C$ & FMEF GATE & & 1 & $08 / 91$ & & 215 & & & Benton & \\
\hline 400 & BUILDING, & TRP & STEFFEN, JIM & STEFFEN, JIM & & & & & Washington & \\
\hline 4702 & OFFICE BUILDING & & 1 & $08 / 82$ & & 2016 & & & Benton & \\
\hline 400 & & $\mathrm{KEH}$ & GONZALES, RI & I TANNER, ROBE & & & & & Washington & \\
\hline 4703 & FFTF CONTROL & & 1 & $08 / 82$ & & 1323 & & & Benton & \\
\hline 400 & BUILDING & TRP & KLOS, DAVID & LOIKA, EDWAR & & & & & Washingtor & \\
\hline $4704 \mathrm{~N}$ & SECURITY & & 1 & $08 / 82$ & & 743 & & & Benton & \\
\hline 400 & MAINTENANCE & $\mathrm{KEH}$ & GONZALES, RI & I TANNER, ROBE & & & & & Washington & \\
\hline $4704 S$ & 400 AREA FIRE & & 1 & $08 / 82$ & 15 & 777 & & & Benton & \\
\hline 400 & STATION & ESQ & GOOD, DONA & GOOD, DONAL & & & & & Washington & \\
\hline 4706 & OFFICE BUILDING & & 1 & $05 / 83$ & 17 & 1735 & & & Benton & \\
\hline 400 & & $\mathrm{KEH}$ & GONZALES, RI & I TANNER, ROBE & & & & & Washington & \\
\hline 4707 & 400 AREA SITE & & 1 & & & 267 & & & Benton & \\
\hline 400 & SUPPORT OFFICE & $\mathrm{KEH}$ & GONZALES, RI & I TANNER, ROBE & & & & & Washington & \\
\hline 4710 & FFTF OFFICE & & 1 & $08 / 82$ & 211 & 3434 & & & Benton & \\
\hline 400 & BUILDING & TRP & KLOS, DAVID & LOIKA, EDWAR & & & & & Washington & \\
\hline $4713 \mathrm{~A}$ & RIGGERS \& & & 1 & & 6 & 517 & & & Benton & \\
\hline 400 & DRIVERS & TRP & KLOS, DAVID & LOIKA, EDWAR & & & & & Washington & \\
\hline $4713 B$ & FFTF & & 1 & $12 / 79$ & 55 & 3070 & & & Benton & \\
\hline 400 & MAINTENANCE & TRP & KLOS, DAVID & LOIKA, EDWAR & & & & & Washington & \\
\hline
\end{tabular}


WHC-SD-SQA-CSWD-501 ReV 0

\begin{tabular}{|c|c|c|c|c|c|c|c|c|c|c|}
\hline Building & Description & $\begin{array}{l}\text { Perfor. } \\
\text { Category } \\
\text { Contractor }\end{array}$ & $\begin{array}{c}\text { Bldg. } \\
\text { Code/Year } \\
\text { Building } \\
\text { Manager }\end{array}$ & $\begin{array}{l}\text { Year Mission } \\
\text { Buildt } \\
\text { Organiztional } \\
\text { Manager }\end{array}$ & $\begin{array}{l}\text { Occupants } \\
\text { (Number) } \\
\text { Hazardous } \\
\text { Materials }\end{array}$ & $\begin{array}{c}\text { Area } \\
\text { m }^{2} \\
\text { SAR } \\
\text { Number }\end{array}$ & $\begin{array}{l}\text { ATC-21 } \\
\text { Rating } \\
\text { Constur } \\
\text { Type }\end{array}$ & $\begin{array}{l}\text { Priority } \\
\text { Score } \\
\text { Struct. } \\
\text { Accidents }\end{array}$ & State & $\begin{array}{c}\text { Number } \\
\text { Stories } \\
\text { NPH } \\
\text { Evaluation }\end{array}$ \\
\hline $4713 C$ & WAREHOUSE & & 1 & $08 / 82$ & & 374 & & & Benton & \\
\hline 400 & & TRP & DOEBLER, ST & LOIKA, EDWAR & & & & & Washington & \\
\hline $4713 \mathrm{D}$ & INTERIM & & & $01 / 81$ & & 587 & & & Benton & \\
\hline 400 & MAINTENANCE & TRP & KLOS, DAVID & LOIKA, EDWAR & & & & & Washington & \\
\hline 400 & $\begin{array}{l}\text { FFTF RIGGING } \\
\text { LOFT }\end{array}$ & TRP & $\begin{array}{c}/ \\
\text { KLOS, DAVID }\end{array}$ & & 11 & 122 & & & $\begin{array}{l}\text { Benton } \\
\text { Washington }\end{array}$ & \\
\hline 400 & $\begin{array}{l}\text { REACTOR } \\
\text { SERVICE }\end{array}$ & TRP & $\begin{array}{c}/ \\
\text { KLOS, DAVID }\end{array}$ & $\begin{array}{l}08 / 82 \\
\text { LOIKA, EDWAR }\end{array}$ & & 4605 & & & $\begin{array}{l}\text { Benton } \\
\text { Washington }\end{array}$ & \\
\hline 400 & $\begin{array}{l}\text { MEDICAL AID } \\
\text { STATION }\end{array}$ & KEH & $\begin{array}{c}\text { / } \\
\text { GONZALES, RI }\end{array}$ & $\begin{array}{l}08 / 81 \\
\text { I TANNER, ROBE }\end{array}$ & & 171 & & & $\begin{array}{l}\text { Benton } \\
\text { Washington }\end{array}$ & \\
\hline 400 & $\begin{array}{l}\text { FFTF } \\
\text { EMERGENCY }\end{array}$ & TRP & $\begin{array}{c}1 \\
\text { KLOS, DAVID }\end{array}$ & $\begin{array}{l}08 / 82 \\
\text { LOIKA, EDWAR }\end{array}$ & 2 & 186 & & & $\begin{array}{l}\text { Benton } \\
\text { Washington }\end{array}$ & \\
\hline $\begin{array}{l}4722 B \\
400\end{array}$ & $\begin{array}{l}\text { CARPENTER } \\
\text { SHOP }\end{array}$ & $\mathrm{KEH}$ & $\begin{array}{c}1 \\
\text { GONZALES; RI }\end{array}$ & $\begin{array}{l}08 / 82 \\
\text { I TANNER, ROBE }\end{array}$ & & 379 & & & $\begin{array}{l}\text { Benton } \\
\text { Washington }\end{array}$ & \\
\hline $\begin{array}{r}4722 \mathrm{C} \\
400\end{array}$ & PAINTERS SHOP & $\mathrm{KEH}$ & $\begin{array}{c}1 \\
\text { DOTSON, PAU }\end{array}$ & $\begin{array}{l}\text { 08/82 } \\
\text { RITTENHOUSE, }\end{array}$ & 1 & 375 & & & $\begin{array}{l}\text { Benton } \\
\text { Washington }\end{array}$ & \\
\hline 400 & $\begin{array}{l}\text { CARPENTER } \\
\text { SUPPLY }\end{array}$ & TRP & $\begin{array}{c}1 \\
\text { KLOS, DAVID }\end{array}$ & LOIKA, EDWAR & & 30 & & & $\begin{array}{l}\text { Benton } \\
\text { Washington }\end{array}$ & \\
\hline 400 & $\begin{array}{l}\text { MAINTENANCE } \\
\text { FLAMMABLE }\end{array}$ & TRP & $\begin{array}{c}/ \\
\text { KLOS, DAVID }\end{array}$ & LOIKA, EDWAR & & 13 & & & $\begin{array}{l}\text { Benton } \\
\text { Washington }\end{array}$ & \\
\hline
\end{tabular}


WHC-SD-SQA-CSWD-501 Rev 0

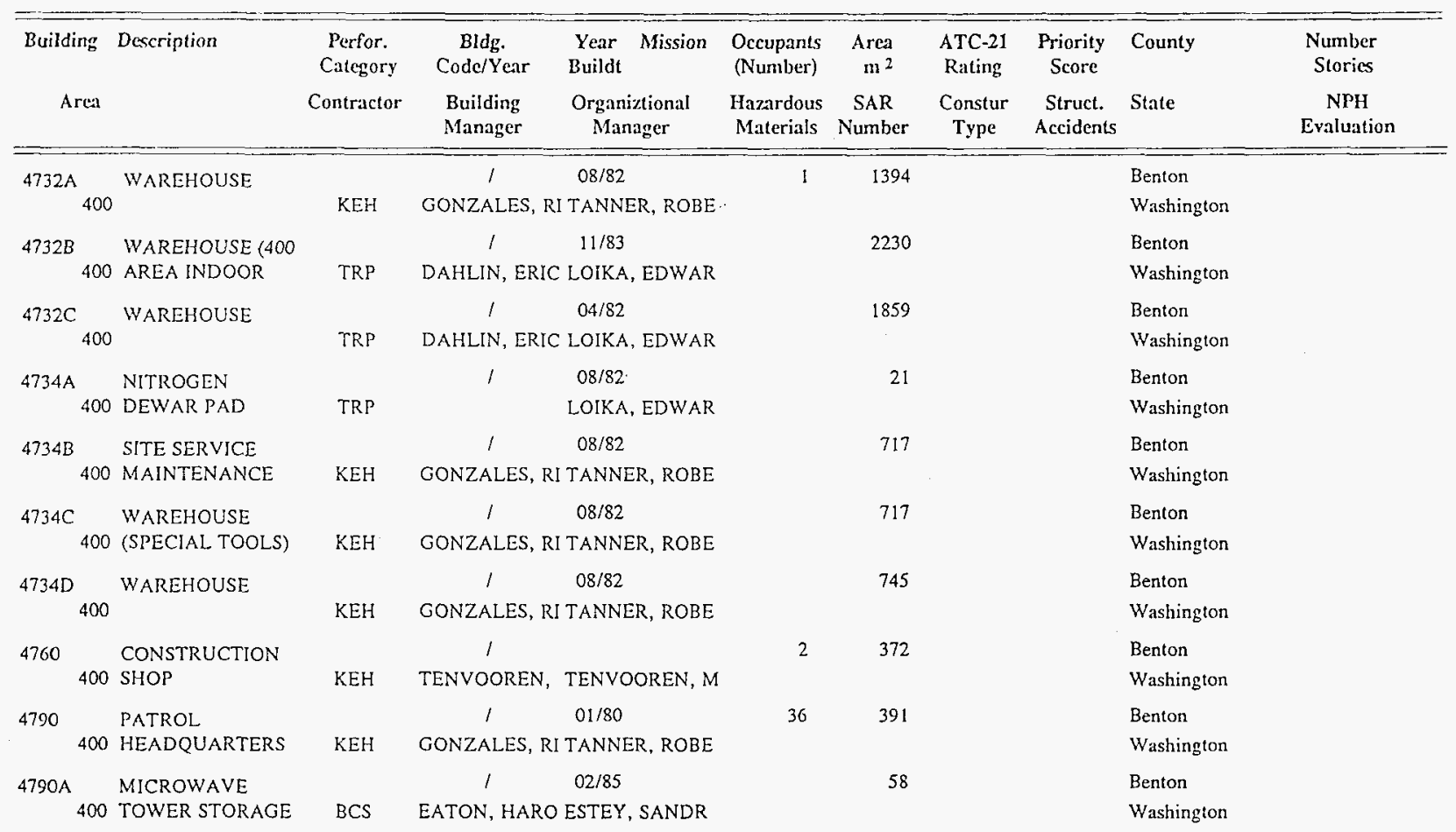


WHC-SD-SQA-CSWD-501 Rev 0

\begin{tabular}{|c|c|c|c|c|c|c|c|c|c|c|}
\hline Building & Description & $\begin{array}{l}\text { Perfor. } \\
\text { Category } \\
\text { Contractor }\end{array}$ & $\begin{array}{l}\text { Bldg. } \\
\text { Code/Year } \\
\text { Building } \\
\text { Manager }\end{array}$ & $\begin{array}{l}\text { Year Mission } \\
\text { Buildt } \\
\text { Organiztional } \\
\text { Manager }\end{array}$ & $\begin{array}{c}\text { Occupants } \\
\text { (Number) } \\
\text { Hazardous } \\
\text { Materials }\end{array}$ & $\begin{array}{c}\text { Area } \\
m^{2} \\
\text { SAR } \\
\text { Number }\end{array}$ & $\begin{array}{l}\text { ATC-2I } \\
\text { Rating } \\
\text { Constur } \\
\text { Type }\end{array}$ & $\begin{array}{l}\text { Priority } \\
\text { Score } \\
\text { Struct. } \\
\text { Accidents }\end{array}$ & State & $\begin{array}{c}\text { Number } \\
\text { Stories } \\
\text { NPH } \\
\text { Evaluation }\end{array}$ \\
\hline $4791 \mathrm{TC}$ & WAREHOUSE & & 1 & $05 / 73$ & & 134 & & & Benton & \\
\hline 400 & & $\mathrm{KEH}$ & GONZALES, RI & MEYER, RICHA & & & & & Washington & \\
\hline 400 & $\begin{array}{l}\text { CONSTRUCTION } \\
\text { SUPPORT }\end{array}$ & TRP & $\begin{array}{c}\prime \\
\text { STEFFEN, JIM }\end{array}$ & $\begin{array}{l}10 / 85 \\
\text { STEFFEN, JIM }\end{array}$ & 2 & 135 & & & $\begin{array}{l}\text { Benton } \\
\text { Washington }\end{array}$ & \\
\hline 400 & $\begin{array}{l}\text { WATER PUMP } \\
\text { HOUSE BUILDING }\end{array}$ & TRP & $\begin{array}{c}\text { KLOS, DAVID } \\
\text { KLO }\end{array}$ & $\begin{array}{l}08 / 82 \\
\text { LOIKA, EDWAR }\end{array}$ & & 146 & & & $\begin{array}{l}\text { Benton } \\
\text { Washington }\end{array}$ & \\
\hline 400 & $\begin{array}{l}\text { SPECIAL TOOLS } \\
\text { WAREHOUSE }\end{array}$ & $\mathrm{KEH}$ & $\begin{array}{c}/ \\
\text { GONZALES, RI }\end{array}$ & $\begin{array}{l}07 / 84 \\
\text { MEYER, RICHA }\end{array}$ & & 465 & & & $\begin{array}{l}\text { Benton } \\
\text { Washington }\end{array}$ & \\
\hline $481 \mathrm{~A}$ & $\begin{array}{l}\text { WATER PUMP } \\
\text { HOUSE BUILDING }\end{array}$ & TRP & $\begin{array}{c}1 \\
\text { KLOS, DAVID }\end{array}$ & $\begin{array}{l}\text { OS/82 } \\
\text { LOIKA, EDWAR }\end{array}$ & & 150 & & & $\begin{array}{l}\text { Benton } \\
\text { Washington }\end{array}$ & \\
\hline 400 & $\begin{array}{l}\text { FLAMMABLE } \\
\text { STORAGE }\end{array}$ & TRP & $\begin{array}{c}\prime \\
\text { DAHLIN, ERIC }\end{array}$ & LOIKA, EDWAR & & 137 & & & $\begin{array}{l}\text { Benton } \\
\text { Washington }\end{array}$ & \\
\hline 400 & $\begin{array}{l}\text { WATER } \\
\text { TREATMENT }\end{array}$ & TRP & $\begin{array}{c}/ \\
\text { STEFFEN, JIM }\end{array}$ & $\begin{array}{l}10 / 85 \\
\text { STEFFEN, JIM }\end{array}$ & & 46 & & & $\begin{array}{l}\text { Benton } \\
\text { Washington }\end{array}$ & \\
\hline 400 & $\begin{array}{l}\text { FFTF IN- } \\
\text { CONTAINMENT }\end{array}$ & TRP & $\begin{array}{c}/ \\
\text { KLOS, DAVID }\end{array}$ & $\begin{array}{l}08 / 82 \\
\text { LOIKA, EDWAR }\end{array}$ & & 784 & & & $\begin{array}{l}\text { Benton } \\
\text { Washington }\end{array}$ & \\
\hline $\begin{array}{l}4842 \mathrm{~A} \\
400\end{array}$ & $\begin{array}{l}\text { SWITCHGEAR } \\
451 B \text { SUBSTATION }\end{array}$ & TRP & $\begin{array}{c}1 \\
\text { KLOS, DAVID }\end{array}$ & LOIKA, EDWAR & & 206 & & & $\begin{array}{l}\text { Benton } \\
\text { Washington }\end{array}$ & \\
\hline $\begin{array}{l}4842 B \\
400\end{array}$ & $\begin{array}{l}\text { SWITCHGEAR } \\
\text { BLDG FOR PUMP }\end{array}$ & TRP & $\begin{array}{c}1 \\
\text { KLOS, DAVID }\end{array}$ & LOIKA, EDWAR & & 28 & & & $\begin{array}{l}\text { Benton } \\
\text { Washington }\end{array}$ & \\
\hline
\end{tabular}


WHC-SD-SQA-CSWD-501 ReV 0

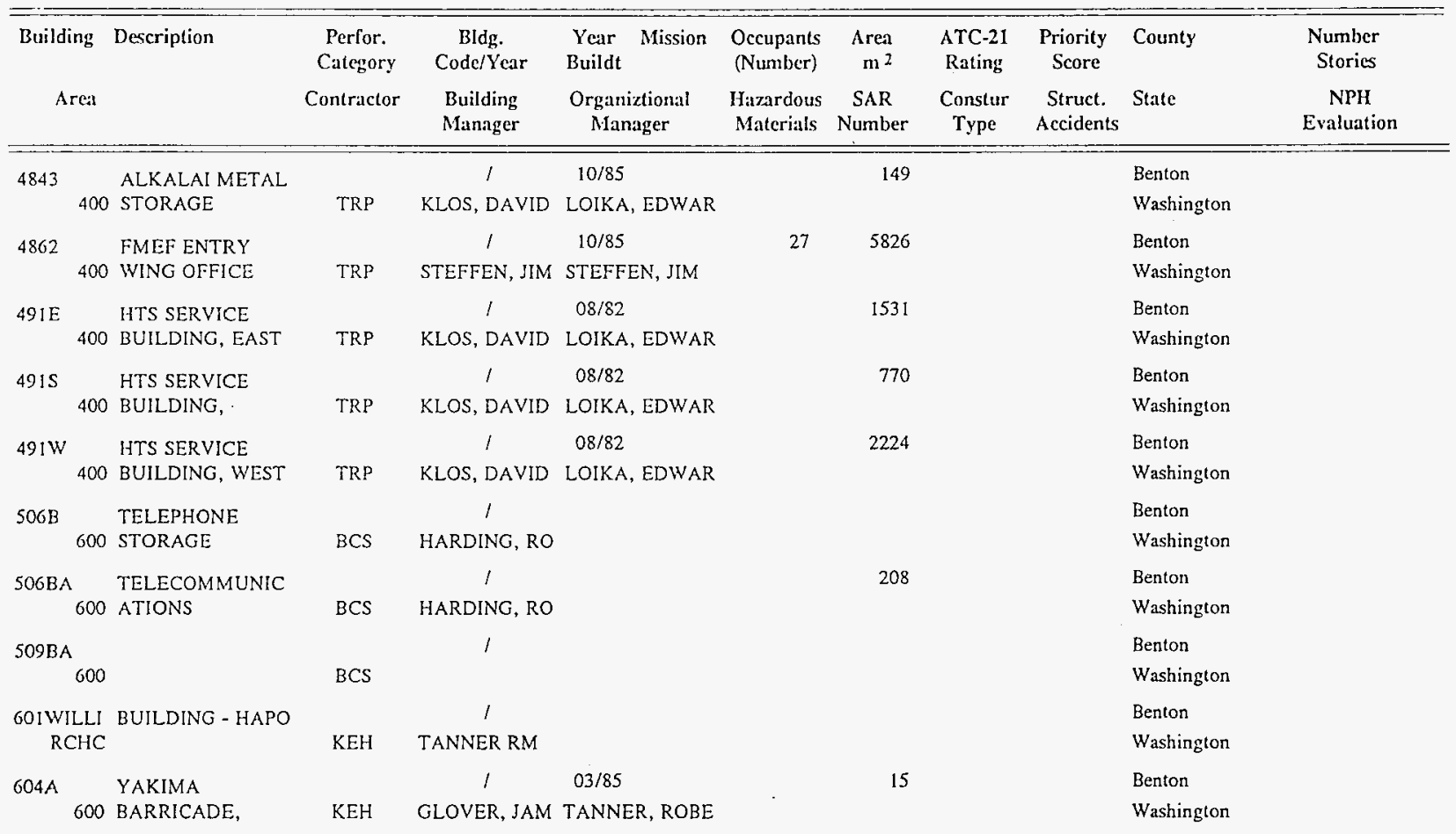


WHC-SD-SQA-CSWD-501 Rev 0

\begin{tabular}{|c|c|c|c|c|c|c|c|c|c|c|c|}
\hline \multirow{2}{*}{\multicolumn{2}{|c|}{$\begin{array}{l}\text { Building } \\
\text { Area }\end{array}$}} & \multirow[t]{2}{*}{ Description } & \multirow{2}{*}{$\begin{array}{c}\text { Perfor. } \\
\text { Category } \\
\text { Contractor }\end{array}$} & \multirow{2}{*}{$\begin{array}{c}\text { Bldg. } \\
\text { Code/Year } \\
\text { Building } \\
\text { Manager }\end{array}$} & \multirow{2}{*}{$\begin{array}{l}\text { Year Mission } \\
\text { Buildt } \\
\text { Organiztional } \\
\text { Manager }\end{array}$} & \multirow{2}{*}{$\begin{array}{c}\text { Occupants } \\
\text { (Number) } \\
\text { Hazardous } \\
\text { Materials }\end{array}$} & \multirow{2}{*}{$\begin{array}{c}\text { Area } \\
m^{2} \\
\text { SAR } \\
\text { Number }\end{array}$} & \multirow{2}{*}{$\begin{array}{c}\text { ATC-21 } \\
\text { Rating } \\
\text { Constur } \\
\text { Type }\end{array}$} & \multirow{2}{*}{$\begin{array}{l}\text { Priority } \\
\text { Score } \\
\text { Struct. } \\
\text { Accidents }\end{array}$} & \multirow{2}{*}{$\begin{array}{l}\text { County } \\
\text { State }\end{array}$} & \multirow{2}{*}{$\begin{array}{c}\text { Number } \\
\text { Stories } \\
\text { NPH } \\
\text { Evaluation }\end{array}$} \\
\hline & & & & & & & & & & & \\
\hline \multirow[t]{2}{*}{$604 \mathrm{~F}$} & & WYE & & 1 & $10 / 59$ & & 7 & & & Benton & \\
\hline & 600 & BARRICADE, & $\mathrm{KEH}$ & MCDOWELL, & TANNER, ROBE & & & & & Washington & \\
\hline \multirow[t]{2}{*}{$604 \mathrm{G}$} & & WYE & & I & $10 / 59$ & & 3 & & & Benton & \\
\hline & 600 & BARRICADE, & $\mathrm{KEH}$ & GONZALES, RI & I TANNER, ROBE & & & & & Washington & \\
\hline \multirow[t]{2}{*}{$604 \mathrm{H}$} & & PATROL UTILITY & & 1 & & & 9 & & & Benton & \\
\hline & 600 & BUILDING & $\mathrm{KEH}$ & GONZALES, RI & TANNER, ROBE & & & & & Washington & \\
\hline \multirow[t]{2}{*}{607} & & BATCH PLANT & & 1 & & & & & & Benton & \\
\hline & 600 & LAB & $\mathrm{KEH}$ & TENVOOREN, & TANNER, ROBE & & & & & Washington & \\
\hline \multirow{2}{*}{609} & & CENTRAL FIRE & & I & $09 / 61$ & 5 & 1004 & & & Benton & \\
\hline & 600 & STATION, I00 & ESQ & GOOD, DONA & GOOD, DONAL & & & & & Washington & \\
\hline \multirow[t]{2}{*}{$609 \mathrm{~A}$} & & FIRE STATION, & & 1 & $06 / 65$ & 23 & 776 & & & Benton & \\
\hline & 600 & 200 AREAS & ESQ & GOOD, DONA & GOOD, DONAL & & & & & Washington & \\
\hline \multirow[t]{2}{*}{$609 \mathrm{C}$} & & FIRE & & 1 & $08 / 85$ & 23 & 186 & & & Benton & \\
\hline & 600 & DEPARTMENT & ESQ & GOOD, DONA & GOOD, DONAL & & & & & Washington & \\
\hline \multirow[t]{2}{*}{6090} & & FIRE & & 1 & $08 / 85$ & & & & & Benton & \\
\hline & 600 & DEPARTMENT & ESQ & & GOOD, DONAL & & & . & & Washington & \\
\hline \multirow[t]{2}{*}{ 609E } & & FIRE STATION & & 1 & & & 167 & & & Benton & \\
\hline & 600 & STORAGE & ESQ & GOOD, DONA & GOOD, DONAL & & & & & Washington & \\
\hline \multirow[t]{2}{*}{$609 \mathrm{G}$} & & FIRE ALARM & & I & $11 / 90$ & 14 & 255 & & & Benton & \\
\hline & 600 & AND TESTING & ESQ & GOOD, DONA & GOOD, DONAL & & & & & Washington & \\
\hline
\end{tabular}


WHC-SD-SQA-CSWD-501 ReV 0

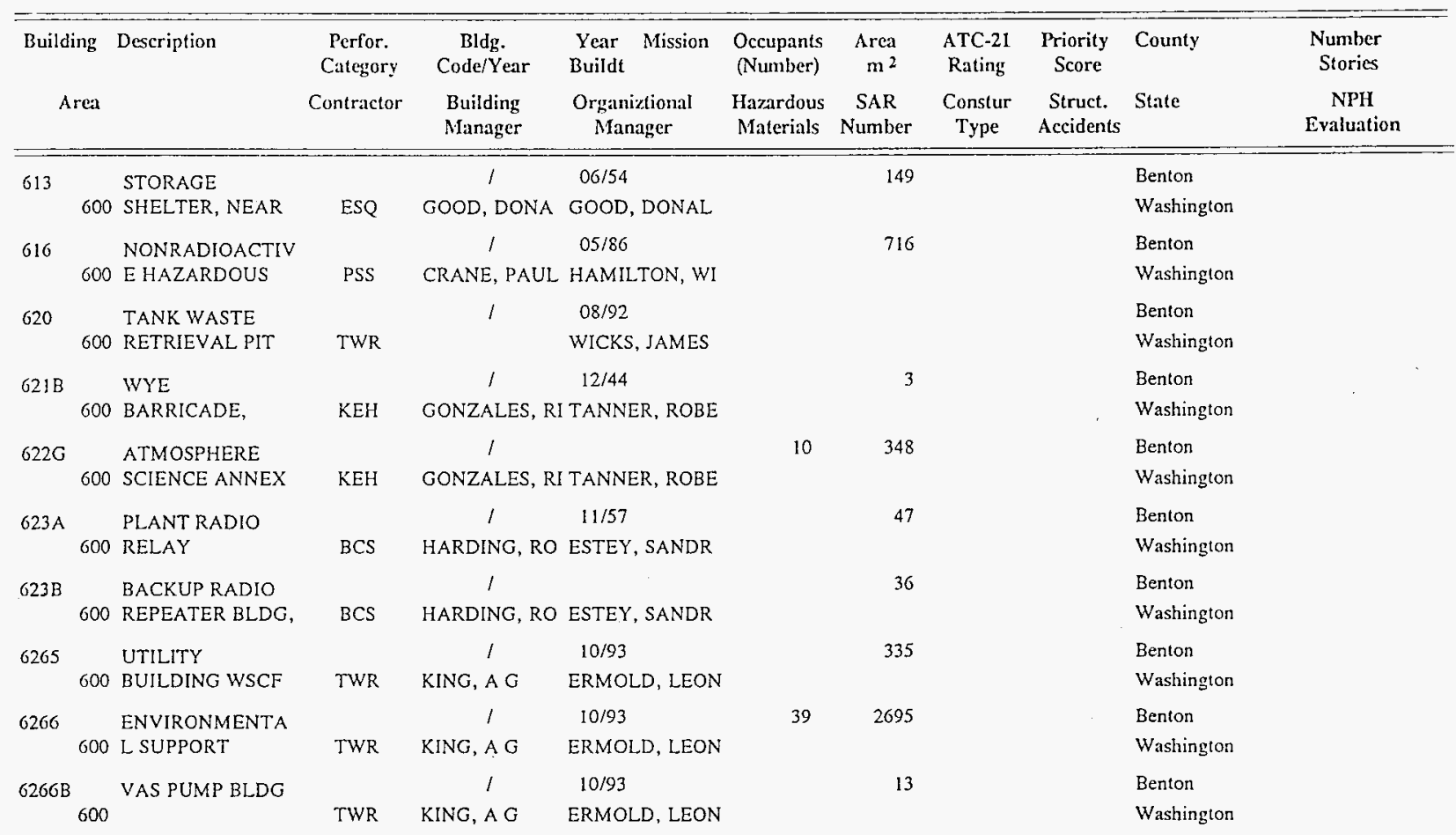


WHC-SD-SQA-CSWD-501 Rev 0

\begin{tabular}{|c|c|c|c|c|c|c|c|c|c|c|c|}
\hline \multicolumn{2}{|c|}{ Building } & \multirow[t]{2}{*}{ Description } & $\begin{array}{l}\text { Perfor, } \\
\text { Category }\end{array}$ & $\begin{array}{l}\text { Bldg. } \\
\text { Code/Year }\end{array}$ & $\begin{array}{l}\text { Year Mission } \\
\text { Buildt }\end{array}$ & $\begin{array}{l}\text { Occupants } \\
\text { (Number) }\end{array}$ & $\begin{array}{c}\text { Area } \\
\mathrm{m}^{2}\end{array}$ & $\begin{array}{l}\text { ATC-21 } \\
\text { Rating }\end{array}$ & $\begin{array}{l}\text { Priority } \\
\text { Score }\end{array}$ & County & $\begin{array}{l}\text { Number } \\
\text { Stories }\end{array}$ \\
\hline \multicolumn{2}{|c|}{ Area } & & Contractor & $\begin{array}{l}\text { Building } \\
\text { Manager }\end{array}$ & $\begin{array}{l}\text { Organiztional } \\
\text { Manager }\end{array}$ & $\begin{array}{l}\text { Hazardous } \\
\text { Materials }\end{array}$ & $\begin{array}{c}\text { SAR } \\
\text { Number }\end{array}$ & $\begin{array}{l}\text { Constur } \\
\text { Type }\end{array}$ & $\begin{array}{l}\text { Struct. } \\
\text { Accidents }\end{array}$ & State & $\begin{array}{c}\text { NPH } \\
\text { Evaluation }\end{array}$ \\
\hline \multirow[t]{2}{*}{6267} & & WSCF COLD & & 1 & $10 / 93$ & & & & & Benton & \\
\hline & 600 & SAMPLE & TWR & KING, A G & ERMOLD, LEON & & & & & Washington & \\
\hline \multirow[t]{2}{*}{6268} & & WSCF SAMPLE & & I & $07 / 95$ & & 223 & & & Benton & \\
\hline & 600 & EQUIPMENT & TWR & $\mathrm{KING}, \mathrm{AG}$ & ERMOLD, LEON & & & & & Washington & \\
\hline \multirow[t]{2}{*}{6269} & & WSCF MOBILE & & I & $10 / 93$ & 6 & 627 & . & & Benton & \\
\hline & 600 & LAB STORAGE & TWR & KING, A G & ERMOLD, LEON & & & & & Washington & \\
\hline \multirow[t]{2}{*}{6270} & & ENVIRONMENTA & & 1 & $10 / 93$ & 9 & & & & Benton & \\
\hline & 600 & L DATA & TWR & KING, A G & ERMOLD, LEON & & & & & Washington & \\
\hline \multirow[t]{2}{*}{6290} & & RIGGING & & 1 & $12 / 89$ & 31 & 804 & & & Benton & \\
\hline & 600 & SERVICES & $\mathrm{KEH}$ & GONZALES, RI & TANNER, ROBE & & & & & Washington & \\
\hline \multirow[t]{2}{*}{6291} & & FUELING & & 1 & $08 / 95$ & & 26 & & & Benton & \\
\hline & 600 & FACILITY & $A D M$ & MINETTE, MI & MEYER, RICHA & & & & & Washington & \\
\hline \multirow[t]{2}{*}{630} & & PLANT & & 1 & & & Il & & & Benton & \\
\hline & 600 & MICROWAVE & $\mathrm{BCS}$ & HARDING, RO & ESTEY, SANDR & & & & & Washington & \\
\hline \multirow[t]{2}{*}{661} & & RIFLE AND & & 1 & $04 / 82$ & & & & & Benton & \\
\hline & 600 & PISTOL RANGE & ESQ & & WALTON, CRAI & & & & & Washington & \\
\hline \multirow[t]{2}{*}{$66 l \mathrm{~A}$} & & TARGET RANGE & & 1 & $04 / 82$ & & 169 & & & Benton & \\
\hline & 600 & CONTROL & $\mathrm{KEH}$ & GONZALES, RI & TANNER, ROBE & & & & & Washington & \\
\hline \multirow[t]{2}{*}{662} & & PATROL & & 1 & $04 / 82$ & 2 & 335 & & & Benton & \\
\hline & 600 & TRAINING & $\mathrm{KEH}$ & GONZALES, RI & TANNER, ROBE & & & & & Washington & \\
\hline
\end{tabular}


WHC-SD-SQA-CSWD-501 ReV 0

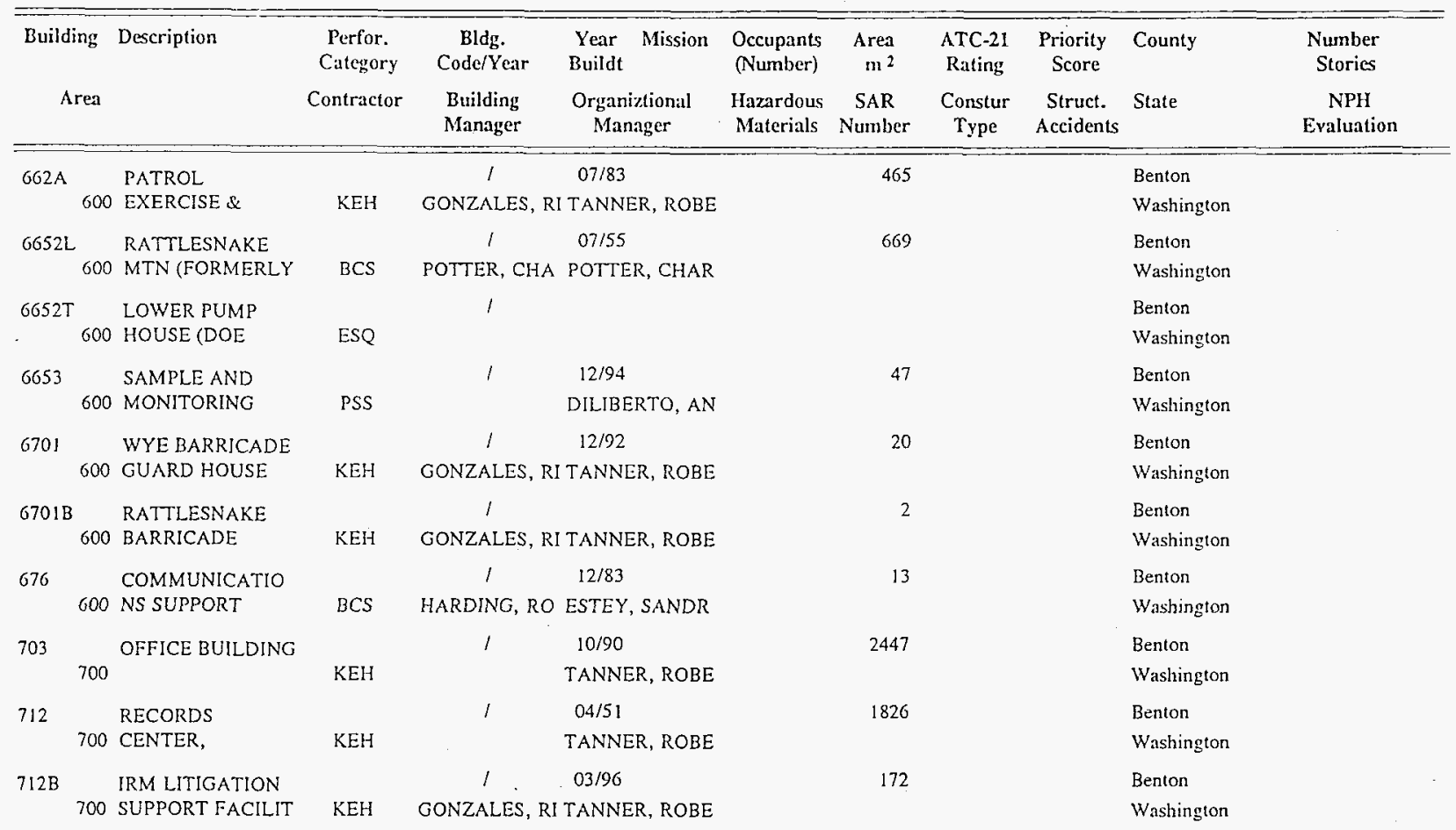


WHC-SD-SQA-CSWD-501 ReV 0

\begin{tabular}{|c|c|c|c|c|c|c|c|c|c|c|}
\hline Building & Description & $\begin{array}{l}\text { Perfor. } \\
\text { Category }\end{array}$ & $\begin{array}{l}\text { Bldg. } \\
\text { Code/Year }\end{array}$ & $\begin{array}{l}\text { Year Mission } \\
\text { Buildt }\end{array}$ & $\begin{array}{l}\text { Occupants } \\
\text { (Number) }\end{array}$ & $\begin{array}{c}\text { Area } \\
\mathrm{m}^{2}\end{array}$ & $\begin{array}{c}\text { ATC-21 } \\
\text { Rating }\end{array}$ & $\begin{array}{l}\text { Priority } \\
\text { Score }\end{array}$ & County & $\begin{array}{c}\text { Number } \\
\text { Storics }\end{array}$ \\
\hline Area & & Contractor & $\begin{array}{l}\text { Building } \\
\text { Manager }\end{array}$ & $\begin{array}{l}\text { Organiztional } \\
\text { Manager }\end{array}$ & $\begin{array}{l}\text { Hazardous } \\
\text { Materials }\end{array}$ & $\begin{array}{c}\text { SAR } \\
\text { Number }\end{array}$ & $\begin{array}{l}\text { Constur } \\
\text { Type }\end{array}$ & $\begin{array}{c}\text { Struct. } \\
\text { Accidents }\end{array}$ & State & $\begin{array}{c}\text { NPH } \\
\text { Evaluation }\end{array}$ \\
\hline \multirow{2}{*}{$\begin{array}{l}712 \mathrm{SWIF} \\
\mathrm{RCHC}\end{array}$} & BUILDING MED & & 1 & & & & & & Benton & \\
\hline & DEN BLD & $\mathrm{KEH}$ & TANNER RM & & & & & & Washington & \\
\hline \multirow[t]{2}{*}{747} & ENVIRONMENTA & & 1 & $06 / 54$ & & 724 & & & Benton & \\
\hline & L HEALTH & KEH & & TANNER, ROBE & & & & & Washington & \\
\hline \multirow[t]{2}{*}{$747 \mathrm{~B}$} & ENVIRONMENTA & & 1 & $05 / 78$ & & 231 & & & Benton & \\
\hline & L HEALTH & $\mathrm{KEH}$ & & TANNER, ROBE & & & & & Washington & \\
\hline \multirow[t]{2}{*}{748} & RADIOSURGERY & & 1 & $10 / 67$ & & 296 & & & Benton & \\
\hline & FACILITY & KEH & & TANNER, ROBE & & & & & Washington & \\
\hline \multirow{2}{*}{$\begin{array}{r}\text { FED BLD } \\
\text { RCHN }\end{array}$} & FEDERAL & & 1 & & & & & & Benton & \\
\hline & BUILDING & $\mathrm{KEH}$ & TANNER RM & & & & & & Washington & \\
\hline \multirow{2}{*}{$\begin{array}{r}\text { MO001 } \\
600\end{array}$} & MOBILE OFFICE & & 1 & & 6 & 125 & & & Benton & \\
\hline & $@$ PTA & KEH & GONZALES, RI & TANNER, ROBE & & & & & Washington & \\
\hline \multirow{2}{*}{$\begin{array}{r}M 0002 \\
600\end{array}$} & MOBILE OFFICE & & 1 & & 5 & 125 & & & Benton & \\
\hline & (10) PTA & $\mathrm{KEH}$ & GONZALES, RI & TANNER, ROBE & & & & & Washington & \\
\hline \multirow{2}{*}{$\begin{array}{l}\text { MO005 } \\
600\end{array}$} & MOBILE OFFICE & & 1 & & & 62 & & & Benton & \\
\hline & (9) HTS PIPEYARD & PSS & GARDNER, M & DILIBERTO, AN & & & & & Washington & \\
\hline \multirow{2}{*}{$\begin{array}{r}\text { MO006 } \\
300\end{array}$} & MOBILE OFFICE & & 1 & & & 62 & & & Benton & \\
\hline & (377 TRL 1) & STS & HUNTER, JAM & BROWN, LARRY & & & & & Washington & \\
\hline \multirow{2}{*}{$\begin{array}{r}\text { MO009 } \\
300\end{array}$} & MOBILE OFFICE & & 1 & & 1 & 62 & & & Benton & \\
\hline & (3745 TRL 1) & $\mathrm{KEH}$ & GONZALES, RI & & & & & & Washington & \\
\hline
\end{tabular}


WHC-SD-SQA-CSWD-501 ReV 0

\begin{tabular}{|c|c|c|c|c|c|c|c|c|c|c|}
\hline Building & Description & $\begin{array}{l}\text { Perfor. } \\
\text { Calegory }\end{array}$ & $\begin{array}{c}\text { Bldg. } \\
\text { Code/Year }\end{array}$ & $\begin{array}{l}\text { Year Mission } \\
\text { Buildt }\end{array}$ & $\begin{array}{l}\text { Occupants } \\
\text { (Number) }\end{array}$ & $\begin{array}{r}\text { Area } \\
\text { In }^{2}\end{array}$ & $\begin{array}{c}\text { ATC-21 } \\
\text { Rating }\end{array}$ & $\begin{array}{c}\text { Priority } \\
\text { Score }\end{array}$ & County & $\begin{array}{c}\text { Number } \\
\text { Stories }\end{array}$ \\
\hline Area & & Contractor & $\begin{array}{l}\text { Building } \\
\text { Manager }\end{array}$ & $\begin{array}{l}\text { Organiztional } \\
\text { Manager }\end{array}$ & $\begin{array}{l}\text { Hazardous } \\
\text { Materials }\end{array}$ & $\begin{array}{c}\text { SAR } \\
\text { Number }\end{array}$ & $\begin{array}{l}\text { Constur } \\
\text { Type }\end{array}$ & $\begin{array}{l}\text { Struct. } \\
\text { Accidents }\end{array}$ & State & $\begin{array}{c}\text { NPH } \\
\text { Evaluation }\end{array}$ \\
\hline $\begin{array}{l}\text { MO011 } \\
200 \mathrm{~W}\end{array}$ & $\begin{array}{l}\text { 200W PATROL HQ } \\
\text { (0) } 234-5 Z\end{array}$ & $\mathrm{KEH}$ & $\begin{array}{c}l \\
\text { GONZALES, }\end{array}$ & I TANNER, ROBE & 89 & 147 & & & $\begin{array}{l}\text { Benton } \\
\text { Washington }\end{array}$ & \\
\hline $\begin{array}{r}\mathrm{MO012} \\
600\end{array}$ & $\begin{array}{l}\text { MOBILE OFFICE } \\
\text { @ GROUT }\end{array}$ & $\mathrm{KEH}$ & $\begin{array}{c}/ \\
\text { DEMPSEY, K }\end{array}$ & I DEMPSEY, KEIT & & 147 & & & $\begin{array}{l}\text { Benton } \\
\text { Washington }\end{array}$ & \\
\hline $\begin{array}{l}\text { MO014 } \\
200 \mathrm{~W}\end{array}$ & $\begin{array}{l}\text { MOBILE OFFICE } \\
\text { (@) } 2704 \mathrm{Z}\end{array}$ & $\mathrm{KEH}$ & $\begin{array}{c}/ \\
\text { GONZALES, }\end{array}$ & I TANNER, ROBE & 6 & 86 & & & $\begin{array}{l}\text { Benton } \\
\text { Washington }\end{array}$ & \\
\hline $\begin{array}{l}\text { MOO15 } \\
200 \mathrm{~W}\end{array}$ & $\begin{array}{l}\text { MOBILE OFFICE } \\
@ 234-5 Z\end{array}$ & $\mathrm{KEH}$ & $\begin{array}{c}\prime \\
\text { GONZALES, }\end{array}$ & I TANNER, ROBE & 2 & 62 & & & $\begin{array}{l}\text { Benton } \\
\text { Washington }\end{array}$ & \\
\hline $\begin{array}{l}\text { MO016 } \\
200 \mathrm{~W}\end{array}$ & $\begin{array}{l}\text { MOBILE OFFICE } \\
@ 234-5 Z\end{array}$ & $\mathrm{KEH}$ & $\begin{array}{c}\prime \\
\text { GONZALES, }\end{array}$ & I TANNER, ROBE & 3 & 62 & & & $\begin{array}{l}\text { Benton } \\
\text { Washington }\end{array}$ & \\
\hline $\begin{array}{l}\text { M0017 } \\
200 \mathrm{~W}\end{array}$ & $\begin{array}{l}\text { MOBILE OFFICE } \\
\text { (1) } 234-5 \mathrm{Z}\end{array}$ & $\mathrm{KEH}$ & $\begin{array}{c}\prime \\
\text { GONZALES, }\end{array}$ & I TANNER, ROBE & 4 & 62 & & & $\begin{array}{l}\text { Benton } \\
\text { Washington }\end{array}$ & \\
\hline $\begin{array}{l}\text { MOO19 } \\
200 \mathrm{E}\end{array}$ & $\begin{array}{l}\text { JANITOR } \\
\text { STGE/OFC @ } 284 \mathrm{E}\end{array}$ & $\mathrm{KEH}$ & $\begin{array}{c}\prime \\
\text { GONZALES, }\end{array}$ & I TANNER, ROBE & & 62 & & & $\begin{array}{l}\text { Benton } \\
\text { Washington }\end{array}$ & \\
\hline $\begin{array}{l}\text { MO021 } \\
200 \mathrm{E}\end{array}$ & $\begin{array}{l}\text { MOBILE OFFICE } \\
\text { (a) } 2101 \mathrm{M}\end{array}$ & KEH & $\begin{array}{c}\prime \\
\text { GONZALES, }\end{array}$ & I TANNER, ROBE & I & 147 & & & $\begin{array}{l}\text { Benton } \\
\text { Washington }\end{array}$ & \\
\hline $\begin{array}{l}\mathrm{MOO23} \\
200 \mathrm{E}\end{array}$ & $\begin{array}{l}\text { MOBILE OFFICE } \\
\text { (3) } 202 \mathrm{~A}\end{array}$ & TRP & $\begin{array}{c}/ \\
\text { HAMRICK, }\end{array}$ & $\begin{array}{l}11 / 92 \\
\text { HAMRICK, DOU }\end{array}$ & 51 & 515 & & & $\begin{array}{l}\text { Benton } \\
\text { Washington }\end{array}$ & \\
\hline $\begin{array}{r}\text { M0026 } \\
300\end{array}$ & $\begin{array}{l}\text { MOBILE OFFICE } \\
(3705 \text { TRL 1) }\end{array}$ & $\mathrm{KEH}$ & $\begin{array}{c}\prime \\
\text { GONZALES, }\end{array}$ & I TANNER, ROBE & & 125 & & & $\begin{array}{l}\text { Benton } \\
\text { Washington }\end{array}$ & \\
\hline
\end{tabular}


WHC-SD-SQA-CSWD-50I Rev 0

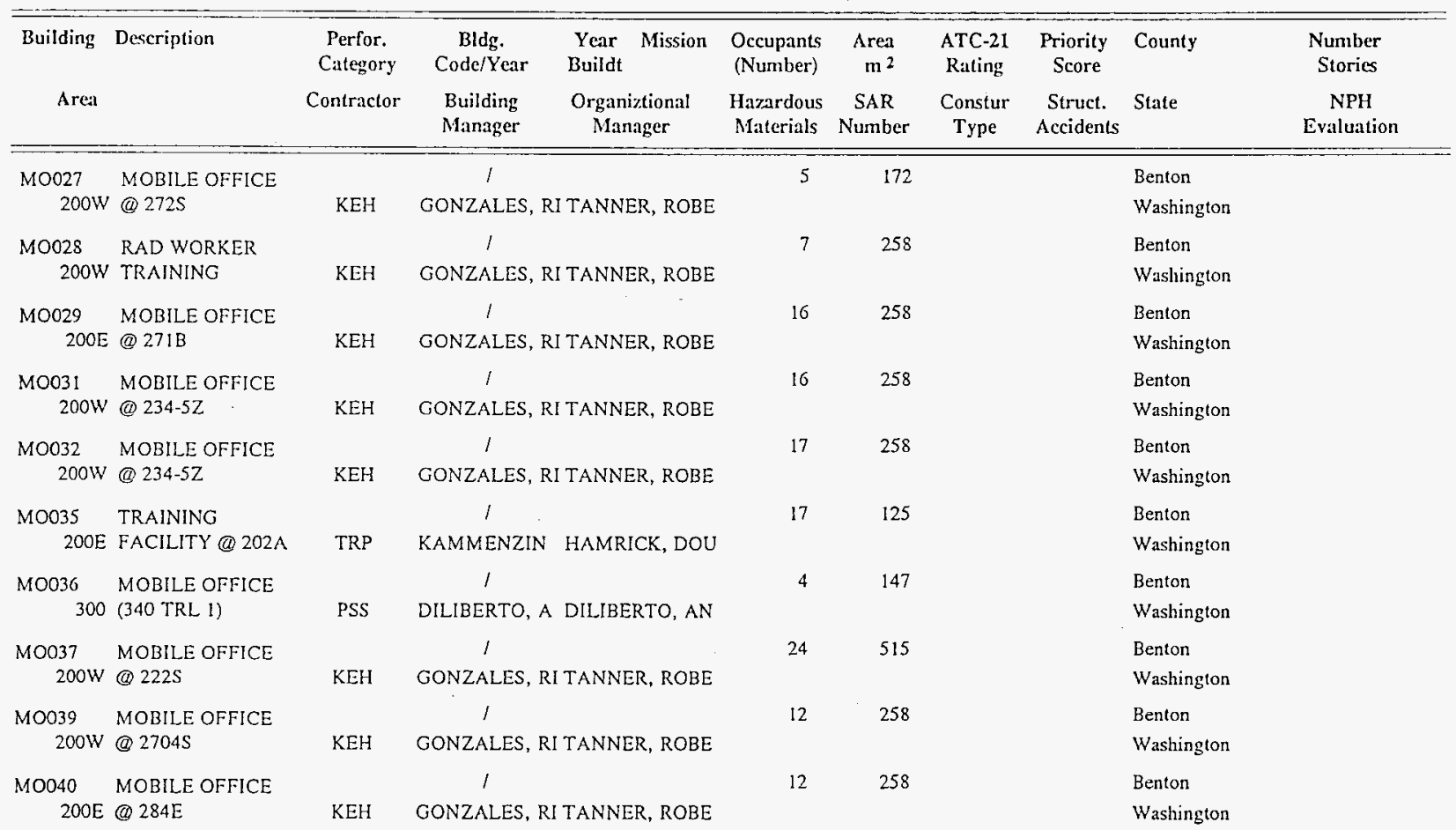


WHC-SD-SQA-CSWD-501 Rev 0

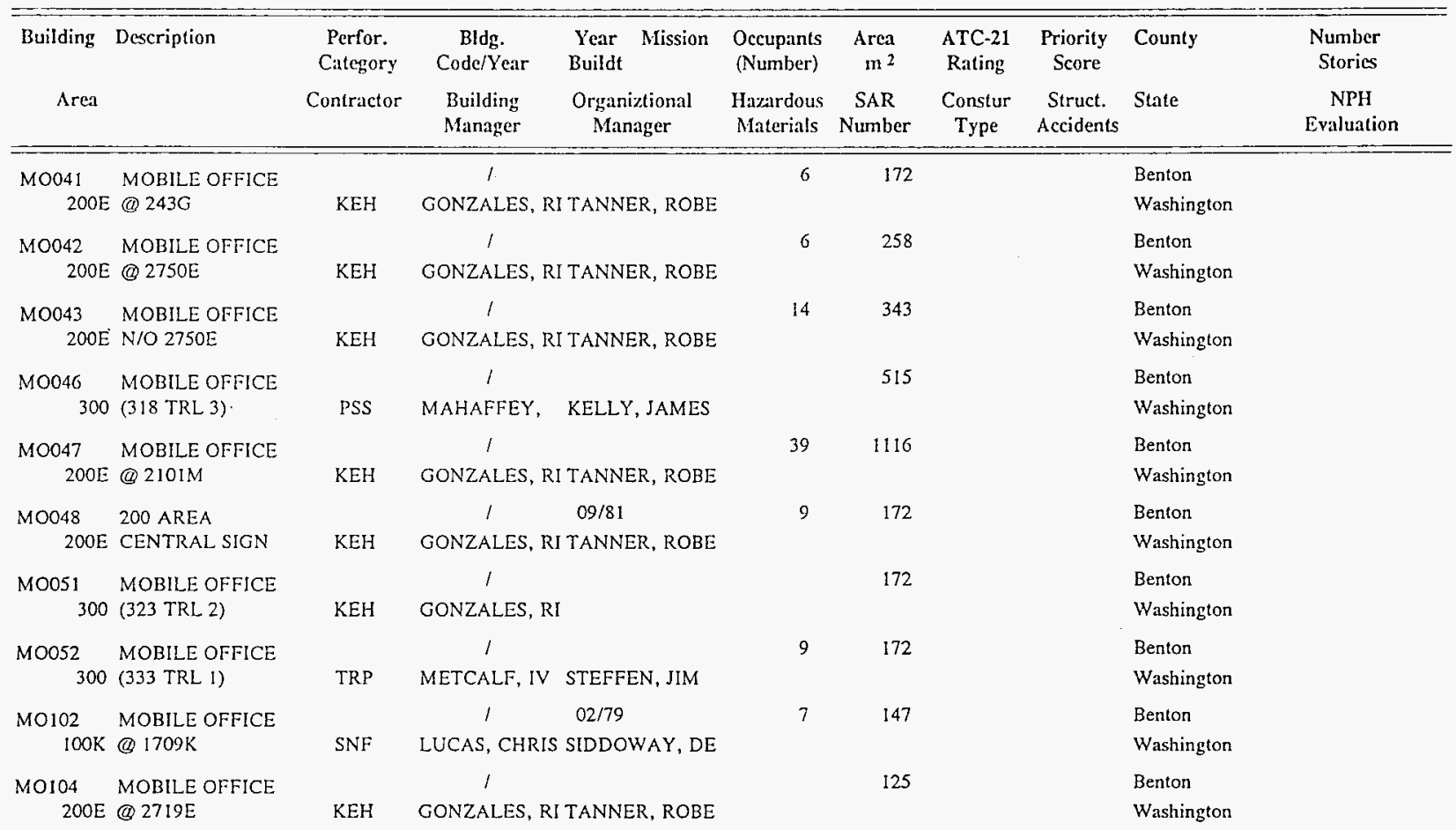


WHC.SD-SQA-CSWD-501 Rev 0

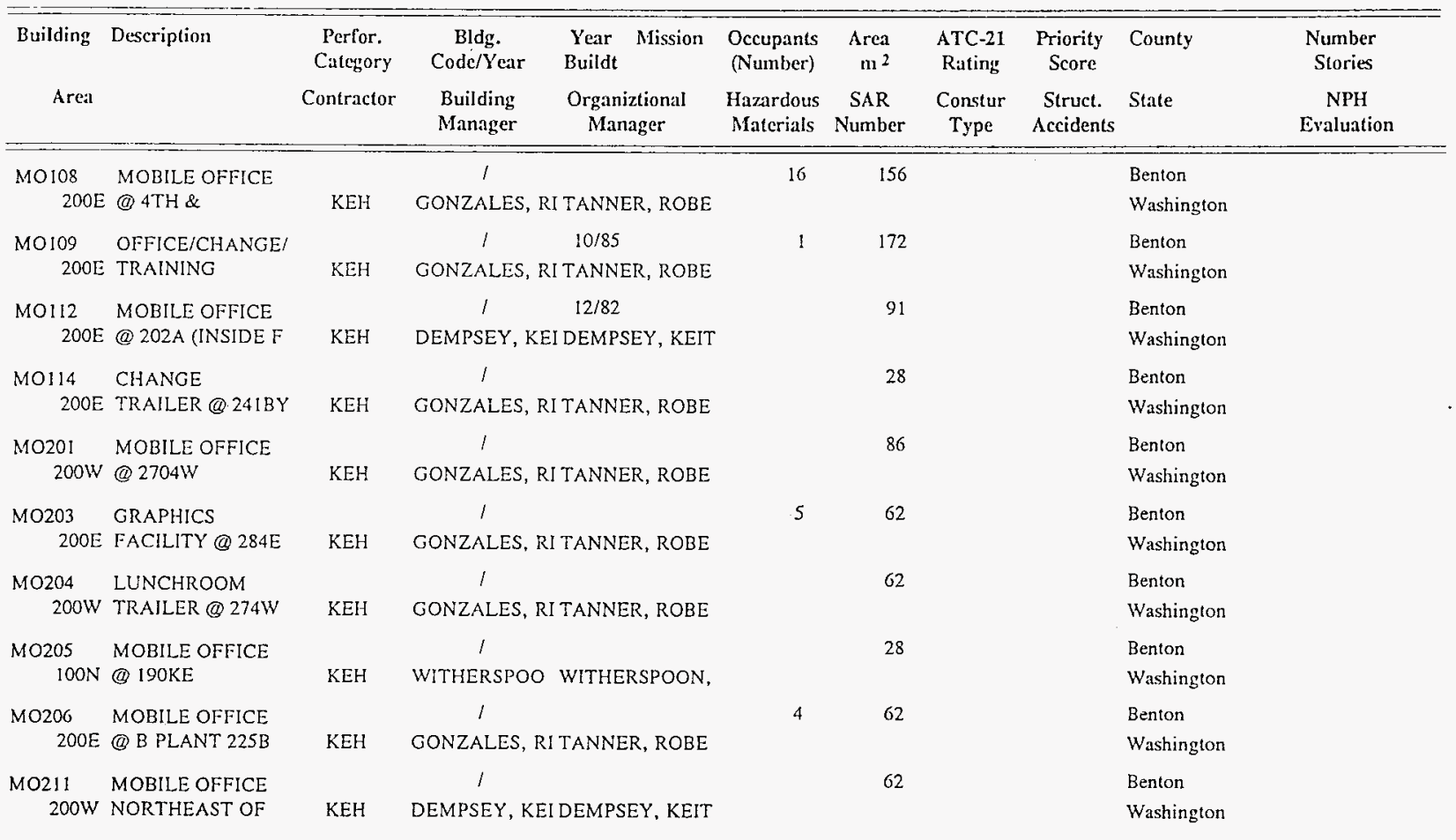


WHC-SD-SQA-CSWD-501 Rev 0

\begin{tabular}{|c|c|c|c|c|c|c|c|c|c|}
\hline Building & Description & $\begin{array}{l}\text { Perfor. } \\
\text { Category }\end{array}$ & $\begin{array}{l}\text { Year Mission } \\
\text { Buildt }\end{array}$ & $\begin{array}{l}\text { Occupants } \\
\text { (Number) }\end{array}$ & $\begin{array}{r}\text { Area } \\
\mathrm{m}^{2}\end{array}$ & $\begin{array}{l}\text { ATC-21 } \\
\text { Rating }\end{array}$ & $\begin{array}{l}\text { Priority } \\
\text { Score }\end{array}$ & County & $\begin{array}{l}\text { Number } \\
\text { Stories }\end{array}$ \\
\hline Area & & Contractor & $\begin{array}{l}\text { Organiztional } \\
\text { Manager }\end{array}$ & $\begin{array}{l}\text { Hazardous } \\
\text { Materials }\end{array}$ & $\begin{array}{c}\text { SAR } \\
\text { Number }\end{array}$ & $\begin{array}{l}\text { Constur } \\
\text { Type }\end{array}$ & $\begin{array}{l}\text { Struct. } \\
\text { Accidents }\end{array}$ & State & $\begin{array}{c}\text { NPH } \\
\text { Evaluation }\end{array}$ \\
\hline $\mathrm{MO} 214$ & PATROL & & 1 & & 62 & & & Benton & \\
\hline $100 \mathrm{~K}$ & BADGEHOUSE & SNF & MILLER, CHA SIDDOWAY, DE & & & & & Washington & \\
\hline MO215 & MOBILE OFFICE & & 1 & & 62 & & & Benton & \\
\hline $200 \mathrm{~W}$ & (1) $2704 \mathrm{~W}$ & $\mathrm{KEH}$ & GONZALES, RI TANNER, ROBE & & & & & Washington & \\
\hline $\mathrm{MO} 221$ & MOBILE OFFICE & & 1 & & 62 & & & Benton & \\
\hline $200 \mathrm{~W}$ & $@ 2704 \mathrm{~W}$ & $\mathrm{KEH}$ & GONZALES, RI TANNER, ROBE & & & & & Washington & \\
\hline $\mathrm{MO} 222$ & GUN CLEANING & & 1 & & 67 & & & Benton & \\
\hline 600 & TRAILER@PTA & $\mathrm{KEH}$ & GONZALES, RI TANNER, ROBE & & & & & Washington & \\
\hline MO223 & CHANGE & & 1 & & 58 & & & Benton & \\
\hline $200 \mathrm{~W}$ & TRAILER N/O & PSS & PYZEL, DONA HAMILTON, WI & & & & & Washington & \\
\hline $\mathrm{MO} 227$ & MOBILE OFFICE & & 1 & 4 & 172 & & & Benton & \\
\hline $200 E$ & (Q) UNSECURED & $\mathrm{KEH}$ & GONZALES, RI TANNER, ROBE & & & & & Washington & \\
\hline $\mathrm{MO} 228$ & SURVEY MOBILE & & 1 & 2 & 172 & & & Benton & \\
\hline $200 \mathrm{E}$ & OFFICE (1) 4TH \& & $\mathrm{KEH}$ & TENVOOREN, TENVOOREN, M & & & & & Washington & \\
\hline MO232 & MOBILE OFFICE & & $1 \quad 09 / 85$ & 6 & 172 & & & Benton & \\
\hline $200 \mathrm{E}$ & $@ 271 B$ & $\mathrm{KEH}$ & GONZALES, RI TANNER, ROBE & & & & & Washington & \\
\hline $\mathrm{MO} 234$ & MOBILE OFFICE & & 1 & 19 & 859 & & & Benton & \\
\hline $200 E$ & (4) $2750 \mathrm{E}$ & $\mathrm{KEH}$ & GONZALES, RI TANNER, ROBE & & & & & Washington & \\
\hline $\begin{array}{l}\text { MO235 } \\
200 \mathrm{~W}\end{array}$ & $\begin{array}{l}\text { MOBILE OFFICE } \\
@ 2722 \mathrm{~W}\end{array}$ & $\mathrm{KEH}$ & $\begin{array}{c}\prime \\
\text { GONZALES, RI TANNER, ROBE }\end{array}$ & 12 & 172 & & & $\begin{array}{l}\text { Benton } \\
\text { Washington }\end{array}$ & \\
\hline
\end{tabular}


WHC-SD-SQA-CSWD-501 Rev 0

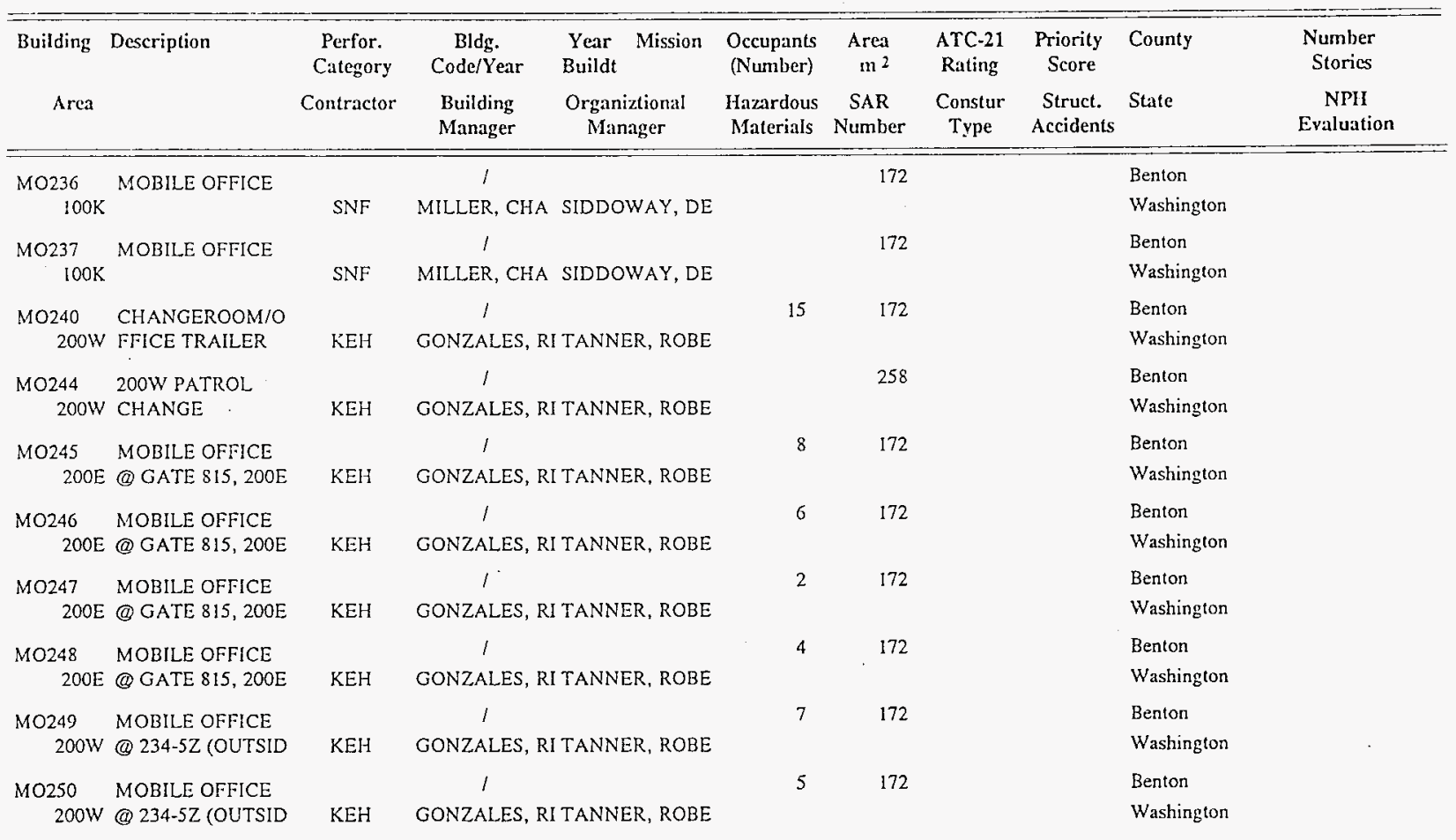


WHC-SD-SQA-CSWD-501 Rev 0

\begin{tabular}{|c|c|c|c|c|c|c|c|c|c|}
\hline Building & Description & $\begin{array}{l}\text { Perfor. } \\
\text { Category } \\
\text { Contractor }\end{array}$ & $\begin{array}{cc}\text { BIdg. } & \text { Year Mission } \\
\text { Code/Year } & \text { Buildt } \\
\text { Building } & \text { Organiztional } \\
\text { Manager } & \text { Manager }\end{array}$ & $\begin{array}{l}\text { Occupants } \\
\text { Number) } \\
\text { Hazardous } \\
\text { Materials }\end{array}$ & $\begin{array}{c}\text { Area } \\
\mathrm{m}^{2} \\
\text { SAR } \\
\text { Number }\end{array}$ & $\begin{array}{l}\text { ATC-21 } \\
\text { Rating } \\
\text { Constur } \\
\text { Type }\end{array}$ & $\begin{array}{l}\text { Priorify } \\
\text { Score } \\
\text { Struct. } \\
\text { Accidents }\end{array}$ & State & $\begin{array}{l}\text { Number } \\
\text { Stories } \\
\text { NPH } \\
\text { Evaluation }\end{array}$ \\
\hline $\begin{array}{l}\text { MO25I } \\
200 E\end{array}$ & $\begin{array}{l}\text { MOBILE OFFICE, } \\
\text { 3RD S/O TRKS ON }\end{array}$ & $\mathrm{KEH}$ & $\begin{array}{c}\prime \\
\text { GONZALES, RI TANNER, ROBE }\end{array}$ & 6 & 172 & & & $\begin{array}{l}\text { Benton } \\
\text { Washington }\end{array}$ & \\
\hline $\begin{array}{l}\mathrm{MO} 252 \\
200 \mathrm{E}\end{array}$ & $\begin{array}{l}\text { MOBILE OFFICE, } \\
\text { 2ND S/O RR TRKS }\end{array}$ & $\mathrm{KEH}$ & $\begin{array}{c}/ \\
\text { GONZALES, RI TANNER, ROBE }\end{array}$ & 7 & 172 & & & $\begin{array}{l}\text { Benton } \\
\text { Washington }\end{array}$ & \\
\hline $\begin{array}{l}\mathrm{MO} 253 \\
200 \mathrm{E}\end{array}$ & $\begin{array}{l}\text { MOBILE OFFICE, } \\
\text { IST S/ORR TRKS }\end{array}$ & $\mathrm{KEH}$ & $\begin{array}{c}/ \\
\text { GONZALES, RI TANNER, ROBE }\end{array}$ & 6 & 172 & & & $\begin{array}{l}\text { Benton } \\
\text { Washington }\end{array}$ & \\
\hline $\begin{array}{r}\mathrm{MO} 254 \\
600\end{array}$ & $\begin{array}{l}\text { MOBILE OFFICE } \\
@ 622 G\end{array}$ & KEH & $\begin{array}{c}/ \\
\text { GONZALES, RI TANNER, ROBE }\end{array}$ & 7 & 172 & & & $\begin{array}{l}\text { Benton } \\
\text { Washington }\end{array}$ & \\
\hline $\begin{array}{l}\mathrm{MO} 255 \\
600\end{array}$ & $\begin{array}{l}\text { MOBILE OFFICE } \\
\text { (Q) } 622 \mathrm{G}\end{array}$ & $\mathrm{KEH}$ & $\begin{array}{c}\text { I } \\
\text { GONZALES, RI TANNER, ROBE }\end{array}$ & 2 & 172 & & & $\begin{array}{l}\text { Benton } \\
\text { Washington }\end{array}$ & \\
\hline $\begin{array}{l}\mathrm{MO} 256 \\
200 \mathrm{E}\end{array}$ & $\begin{array}{l}\text { MOBILE OFFICE } \\
\text { E/O } 2711 \mathrm{E}\end{array}$ & $\mathrm{KEH}$ & $\begin{array}{c}/ \\
\text { GONZALES, RI TANNER, ROBE }\end{array}$ & 8 & 172 & & & $\begin{array}{l}\text { Benton } \\
\text { Washington }\end{array}$ & \\
\hline $\begin{array}{l}\mathrm{MO} 257 \\
200 \mathrm{E}\end{array}$ & $\begin{array}{l}\text { MOBILE OFFICE } \\
\text { E/O } 2711 \mathrm{E}\end{array}$ & $\mathrm{KEH}$ & $\begin{array}{c}\text { / } \\
\text { GONZALES, RI TANNER, ROBE }\end{array}$ & 6 & 172 & & & $\begin{array}{l}\text { Benton } \\
\text { Washington }\end{array}$ & \\
\hline $\begin{array}{r}\text { MO258 } \\
300\end{array}$ & $\begin{array}{l}\text { MOBILE OFFICE } \\
\text { Q FMIT }\end{array}$ & $\mathrm{KEH}$ & $\begin{array}{l}/ \\
\text { GONZALES, RI TANNER, ROBE }\end{array}$ & & 172 & & & $\begin{array}{l}\text { Benton } \\
\text { Washington }\end{array}$ & \\
\hline $\begin{array}{r}\text { MO259 } \\
300\end{array}$ & $\begin{array}{l}\text { MOBILE OFFICE } \\
@ \text { FMIT }\end{array}$ & $\mathrm{KEH}$ & $\begin{array}{l}\text { I } \\
\text { GONZALES, RI TANNER, ROBE }\end{array}$ & 9 & 172 & & & $\begin{array}{l}\text { Benton } \\
\text { Washington }\end{array}$ & \\
\hline $\begin{array}{l}\mathrm{MO} 260 \\
400\end{array}$ & $\begin{array}{l}\text { MOBILE OFFICE } \\
\text { (Q) FMIT }\end{array}$ & $\mathrm{KEH}$ & $\begin{array}{c}\text { I } \\
\text { GONZALES, RI TANNER, ROBE }\end{array}$ & & 172 & & & $\begin{array}{l}\text { Benton } \\
\text { Washington }\end{array}$ & \\
\hline
\end{tabular}


WHC-SD-SQA-CSWD-501 ReV 0

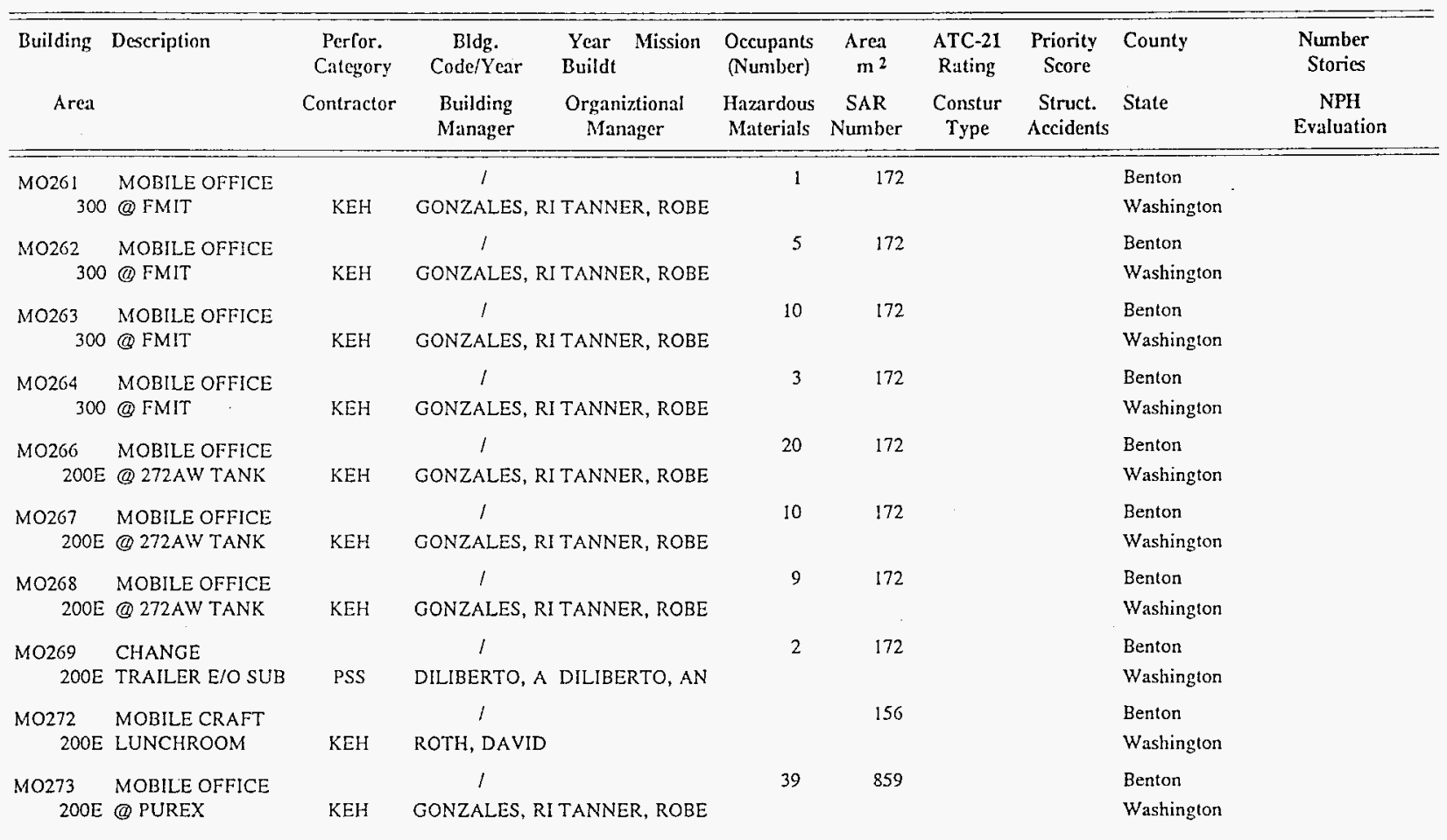


WHC.SD-SQA-CSWO-501 Rev 0

\begin{tabular}{|c|c|c|c|c|c|c|c|c|c|}
\hline Building & Description & $\begin{array}{l}\text { Perfor. } \\
\text { Categrory } \\
\text { Contractor }\end{array}$ & $\begin{array}{l}\text { Year Mission } \\
\text { Buildt } \\
\text { Organiztional } \\
\text { Manager }\end{array}$ & $\begin{array}{l}\text { Occupants } \\
\text { (Number) } \\
\text { Hazardous } \\
\text { Materials }\end{array}$ & $\begin{array}{c}\begin{array}{c}\text { Area } \\
\text { m }^{2} \\
\text { SAR } \\
\text { Number }\end{array}\end{array}$ & $\begin{array}{l}\text { ATC-21 } \\
\text { Rating } \\
\text { Constur } \\
\text { Type }\end{array}$ & $\begin{array}{l}\text { Priorify } \\
\text { Score } \\
\text { Struct. } \\
\text { Accidents }\end{array}$ & State & $\begin{array}{c}\text { Number } \\
\text { Stories } \\
\text { NPH } \\
\text { Evaluation }\end{array}$ \\
\hline $\begin{array}{l}\mathrm{MO} 276 \\
200 \mathrm{E}\end{array}$ & $\begin{array}{l}\text { MOBILE OFFICE } \\
\text { (d) } 2753 \mathrm{E}\end{array}$ & $\mathrm{KEH}$ & $\begin{array}{l}\prime \\
\text { GONZALES, RI TANNER, ROBE }\end{array}$ & & 1374 & & & $\begin{array}{l}\text { Benton } \\
\text { Washington }\end{array}$ & \\
\hline $\begin{array}{l}\mathrm{MO} 277 \\
200 \mathrm{E}\end{array}$ & $\begin{array}{l}\text { MOBILE OFFICE } \\
@ 2753 \mathrm{E}\end{array}$ & $\mathrm{KEH}$ & $\begin{array}{c}/ \\
\text { GONZALES, RI TANNER, ROBE }\end{array}$ & $l$ & 1374 & & & $\begin{array}{l}\text { Benton } \\
\text { Washington }\end{array}$ & \\
\hline $\begin{array}{l}\text { MO278 } \\
200 \mathrm{~W}\end{array}$ & $\begin{array}{l}\text { MOBLLE OFFICE } \\
\text { W/O } 275 \mathrm{~W}\end{array}$ & PSS & $\begin{array}{l}\text { / } \\
\text { HAMILTON, W HAMILTON, WI }\end{array}$ & 51 & 859 & & & $\begin{array}{l}\text { Benton } \\
\text { Washington }\end{array}$ & \\
\hline $\begin{array}{l}\text { MO279 } \\
200 \mathrm{~W}\end{array}$ & $\begin{array}{l}\text { MOBILE OFFICE } \\
\text { W/O } 275 W\end{array}$ & PSS & $\begin{array}{l}/ \\
\text { HAMILTON, W HAMILTON, WI }\end{array}$ & 55 & 859 & & & $\begin{array}{l}\text { Benton } \\
\text { Washington }\end{array}$ & \\
\hline $\begin{array}{l}\mathrm{MO280} \\
600\end{array}$ & $\begin{array}{l}\text { MOBILE OFFICE } \\
@ \text { WSCF SITE }\end{array}$ & $\mathrm{KEH}$ & $\begin{array}{c}\text { / } \\
\text { GONZALES, RI TANNER, ROBE }\end{array}$ & 31 & 859 & & & $\begin{array}{l}\text { Benton } \\
\text { Washington }\end{array}$ & \\
\hline $\begin{array}{l}\mathrm{MO} 281 \\
200 \mathrm{~W}\end{array}$ & $\begin{array}{l}\text { MOBILE OFFICE } \\
\text { @272WA }\end{array}$ & $\mathrm{KEH}$ & $\begin{array}{c}/ \\
\text { GONZALES, RI TANNER, ROBE }\end{array}$ & 84 & 1374 & & & $\begin{array}{l}\text { Benton } \\
\text { Washington }\end{array}$ & \\
\hline $\begin{array}{l}\mathrm{MO} 282 \\
200 \mathrm{E}\end{array}$ & $\begin{array}{l}\text { MOBILE OFFICE } \\
\text { (G) GROUT }\end{array}$ & $\mathrm{KEH}$ & $\begin{array}{l}\text { I } \\
\text { GONZALES, RI TANNER, ROBE }\end{array}$ & 5 & 172 & & & $\begin{array}{l}\text { Benton } \\
\text { Washington }\end{array}$ & \\
\hline $\begin{array}{l}\mathrm{MO} 283 \\
200 \mathrm{E}\end{array}$ & $\begin{array}{l}\text { MOBILE OFFICE } \\
\text { (Q) GROUT }\end{array}$ & $\mathrm{KEH}$ & $\begin{array}{c}\text { I } \\
\text { GONZALES, RI TANNER, ROBE }\end{array}$ & 11 & 172 & & & $\begin{array}{l}\text { Benton } \\
\text { Washington }\end{array}$ & \\
\hline $\begin{array}{r}\mathrm{MO} 284 \\
200 \mathrm{E}\end{array}$ & $\begin{array}{l}\text { MOBILE OFFICE } \\
\text { @ GROUT }\end{array}$ & $\mathrm{KEH}$ & $\begin{array}{l}l \\
\text { GONZALES, RI TANNER, ROBE }\end{array}$ & 5 & 172 & & & $\begin{array}{l}\text { Benton } \\
\text { Washington }\end{array}$ & \\
\hline $\begin{array}{l}\text { MO285 } \\
200 \mathrm{E}\end{array}$ & $\begin{array}{l}\text { MOBILE OFFICE } \\
\text { @ } 2753 E\end{array}$ & $\mathrm{KEH}$ & $\begin{array}{c}l \\
\text { GONZALES, RI TANNER, ROBE }\end{array}$ & 55 & 1030 & & & $\begin{array}{l}\text { Benton } \\
\text { Washington }\end{array}$ & \\
\hline
\end{tabular}


WHC-SD-SQA-CSWD-501 Rev 0

\begin{tabular}{|c|c|c|c|c|c|c|c|c|c|}
\hline Building & Description & $\begin{array}{l}\text { Perfor. } \\
\text { Category }\end{array}$ & $\begin{array}{l}\text { Year Mission } \\
\text { Buildt }\end{array}$ & $\begin{array}{l}\text { Occupants } \\
\text { (Number) }\end{array}$ & $\begin{array}{r}\text { Area } \\
\mathrm{m}^{2}\end{array}$ & $\begin{array}{l}\text { ATC-21 } \\
\text { Rating }\end{array}$ & $\begin{array}{c}\text { Priority } \\
\text { Score }\end{array}$ & County & $\begin{array}{l}\text { Number } \\
\text { Stories }\end{array}$ \\
\hline Area & & Contractor & $\begin{array}{l}\text { Organiztional } \\
\text { Manager }\end{array}$ & $\begin{array}{l}\text { Hazardous } \\
\text { Materials }\end{array}$ & $\begin{array}{c}\text { SAR } \\
\text { Number }\end{array}$ & $\begin{array}{l}\text { Constur } \\
\text { Type }\end{array}$ & $\begin{array}{l}\text { Struct. } \\
\text { Accidents }\end{array}$ & Stale & $\begin{array}{c}\text { NPH } \\
\text { Evaluation }\end{array}$ \\
\hline $\mathrm{MO} 286$ & MOBILE OFFICE & & 1 & 34 & 1030 & & & Benton & \\
\hline $200 \mathrm{E}$ & (Q) $2727 \mathrm{E}$ & $\mathrm{KEH}$ & GONZALES, RI TANNER, ROBE & & & & & Washington & \\
\hline $\mathrm{MO} 287$ & MOBILE OFFICE & & 1 & 47 & 1030 & & & Benton & \\
\hline $200 \mathrm{~W}$ & (1) $275 \mathrm{~W}$ & $\mathrm{KEH}$ & GONZALES, RI TANNER, ROBE & & & & & Washington & \\
\hline MO288 & MOBILE CHANGE & & $1 \quad 04 / 94$ & & 36 & & & Benton & \\
\hline $200 \mathrm{~W}$ & FACILITY@ & PSS & CRANE, PAUL HAMILTON, WI & & & & & Washington & \\
\hline MO289 & MOBILE CHANGE & & $/ \quad 04 / 94$ & 1 & 36 & & & Benton & \\
\hline $200 \mathrm{~W}$ & FACILITY@224T & PSS & CRANE, PAUL HAMILTON, WI & & & & & Washington & \\
\hline MO290 & MOBILE & & 1 & 1 & 172 & & & Benton & \\
\hline $200 \mathrm{~W}$ & COMPUTER HUB & $\mathrm{KEH}$ & GONZALES, RI TANNER, ROBE & & & & & Washington & \\
\hline MO291 & MOBILE OFFICE & & 1 & 42 & 859 & & & Benton & \\
\hline $200 \mathrm{~W}$ & NEAR 27045 & $\mathrm{KEH}$ & GONZALES, RI TANNER, ROBE & & & & & Washington & \\
\hline $\mathrm{MO} 292$ & MOBILE OFFICE & & 1 & 35 & 859 & & & Benton & \\
\hline 600 & (1) WSCF (E) 6270 & KEH & GONZALES, RI TANNER, ROBE & & & & & Washington & \\
\hline $\mathrm{MO} 293$ & MOBILE OFFICE & & 1 & 54 & 859 & & & Benton & \\
\hline $100 K$ & @ 1725K (SPENT F & SNF & BLEHM, LORI SIDDOWAY, DE & & & & & Washington & \\
\hline $\mathrm{MO} 294$ & MOBILE OFFICE & & 1 & 65 & 1374 & & & Benton & \\
\hline $200 \mathrm{E}$ & $@ 2727 \mathrm{E}$ & $\mathrm{KEH}$ & GONZALES, RI TANNER, ROBE & & & & & Washington & \\
\hline MO295 & FEMALE & & 1 & & 71 & & & Benton & \\
\hline $200 \mathrm{~W}$ & CHANGE MOBILE & TWR & WICKS, JAMES WICKS, JAMES & & & & & Washington & \\
\hline
\end{tabular}


WHC-SD-SQA-CSWD-501 ReV 0

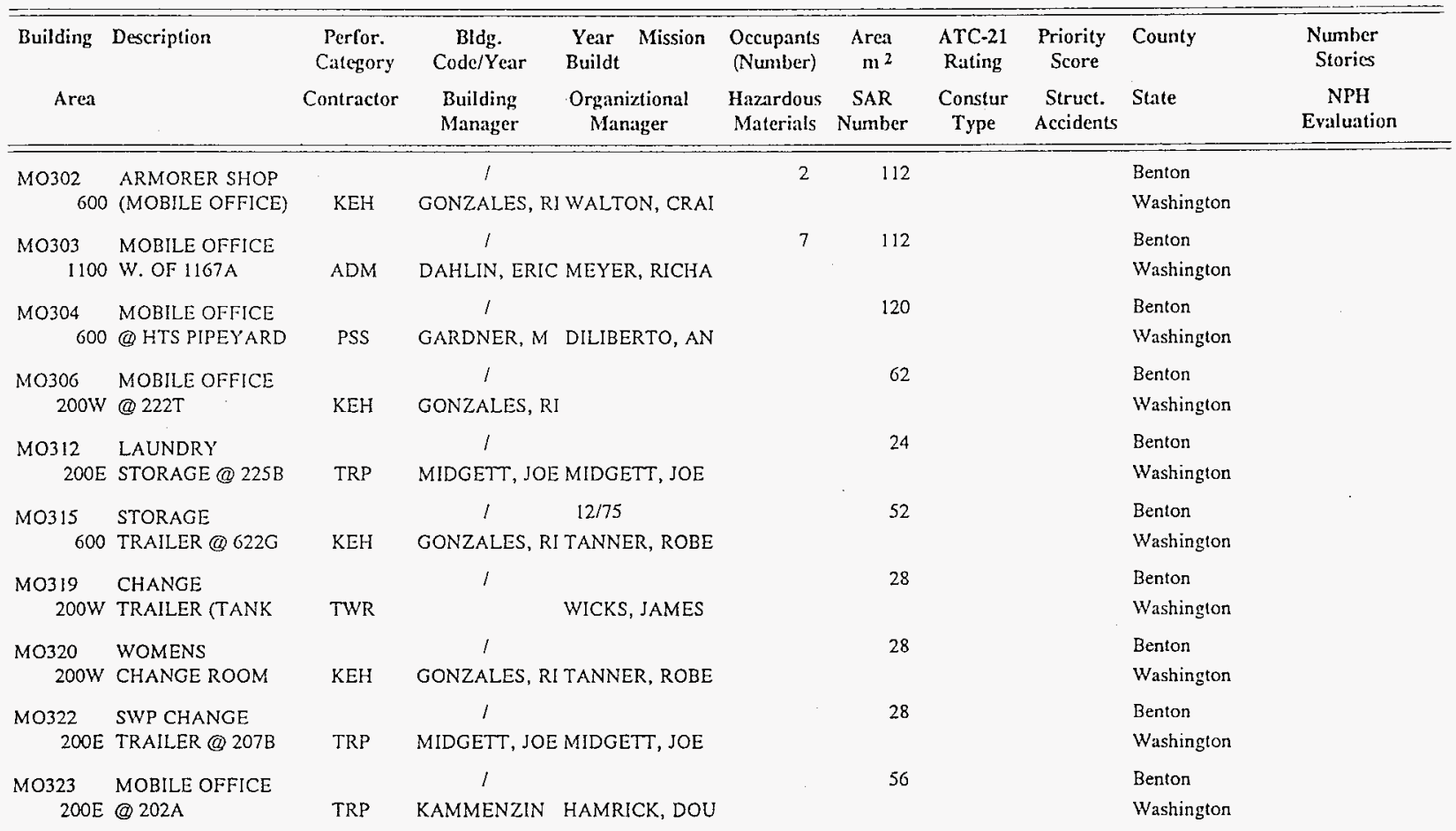


WHC-SD-SQA-CSWD-501 Rev 0

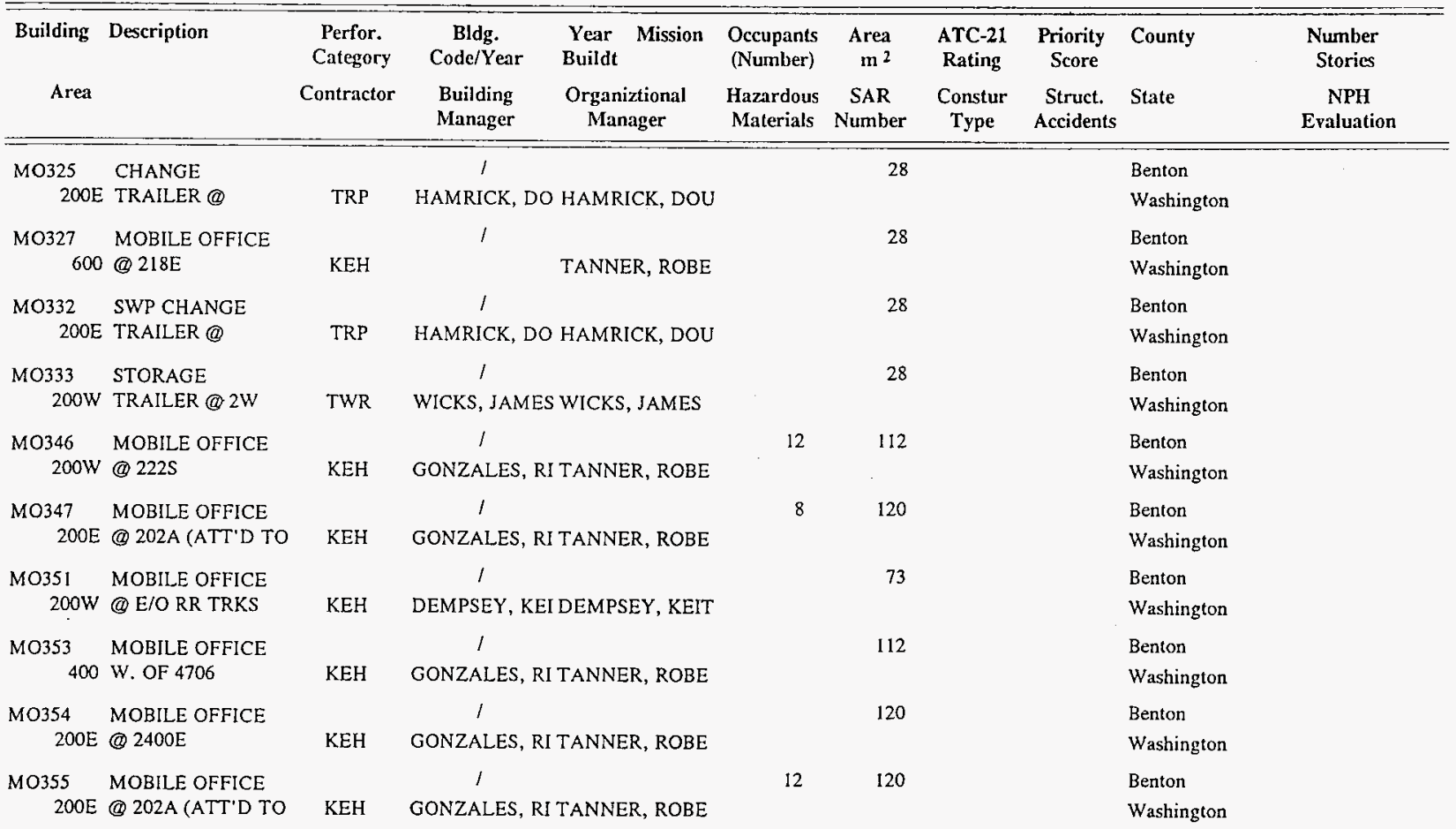


WHC-SD-SQA-CSWD-501 Rev 0

\begin{tabular}{|c|c|c|c|c|c|c|c|c|c|}
\hline Building & Description & $\begin{array}{c}\text { Perfor. } \\
\text { Category } \\
\text { Contractor }\end{array}$ & $\begin{array}{l}\text { Year Mission } \\
\text { Buildt } \\
\text { Organiztional } \\
\text { Manager }\end{array}$ & $\begin{array}{l}\text { Occupants } \\
\text { (Number) } \\
\text { Hazardous } \\
\text { Materials }\end{array}$ & $\begin{array}{c}\text { Area } \\
\mathrm{m}^{2} \\
\text { SAR } \\
\text { Number }\end{array}$ & $\begin{array}{l}\text { ATC-21 } \\
\text { Rating } \\
\text { Constur } \\
\text { Type }\end{array}$ & $\begin{array}{l}\text { Priority } \\
\text { Score } \\
\text { Struct. } \\
\text { Accidents }\end{array}$ & State & $\begin{array}{c}\text { Number } \\
\text { Stories } \\
\text { NPH } \\
\text { Evaluation }\end{array}$ \\
\hline $\mathrm{MO} 363$ & MOBILE OFFICE & & $\begin{array}{ll}1 & 05 / 83\end{array}$ & & 18 & & & Benton & \\
\hline $200 \mathrm{~W}$ & (Q)241SX TANK & TWR & GONZALES, RI WICKS, JAMES & & & & & Washington & \\
\hline MO368 & PATROL & & 1 & & 112 & & & Benton & \\
\hline 600 & TRAINING & $\mathrm{KEH}$ & GONZALES, RI TANNER, ROBE & & & & & Washington & \\
\hline $\mathrm{MO} 369$ & MOBILE OFFICE & & 1 & & 172 & & & Benton & \\
\hline 3000 & NEAR I262 BUILDI & $\mathrm{KEH}$ & TANNER, ROBE & & & & & Washington & \\
\hline $\mathrm{MO} 370$ & MOBILE OFFICE & & 1 & & 10 & & & Benton & \\
\hline 3000 & @2400 STEVENS & $\mathrm{KEH}$ & HOLLADAY, J FOUCAULT, DA & & & & & Washington & \\
\hline $\mathrm{MO} 371$ & D\&D FEMALE & & 1 & & 46 & & & Benton & \\
\hline $200 W$ & CHANGE & $\mathrm{KEH}$ & GONZALES, RI TANNER, ROBE & & & & & Washington & \\
\hline $\mathrm{MO} 376$ & FIELD TRAILER & & 1 & & 22 & & & Benton & - \\
\hline 600 & $@$ KEH SLAB & $\mathrm{KEH}$ & FOUCAULT, DA & & & & & Washington & \\
\hline $\mathrm{MO} 377$ & MOBILE OFFICE & & 1 & 4 & 46 & & & Benton & \\
\hline $200 \mathrm{~W}$ & (C) $275 \mathrm{~W}$ & $\mathrm{KEH}$ & GONZALES, RI TANNER, ROBE & & & & & Washington & \\
\hline $\mathrm{MO} 378$ & MOBILE OFFICE & & 1 & & 172 & & & Benton & \\
\hline 400 & (TRL 100) W. OF & $\mathrm{KEH}$ & GONZALES, RI TANNER, ROBE & & & & & Washington & \\
\hline MO379 & MOBILE OFFICE & & 1 & & 172 & & & Benton & \\
\hline 400 & (TRL I02) W. OF & $\mathrm{KEH}$ & GONZALES, RI TANNER, ROBE & & & & & Washington & \\
\hline $\mathrm{MO} 382$ & MOBILE OFFICE & & I & 7 & 156 & & & Benton & \\
\hline $100 \mathrm{~K}$ & AKA: $1721 \mathrm{~K}$ & SNF & KAVIANI, MA SIDDOWAY, DE & & & & & Washington & \\
\hline
\end{tabular}


WHC-SD-SQA-CSWO-501 Rev 0

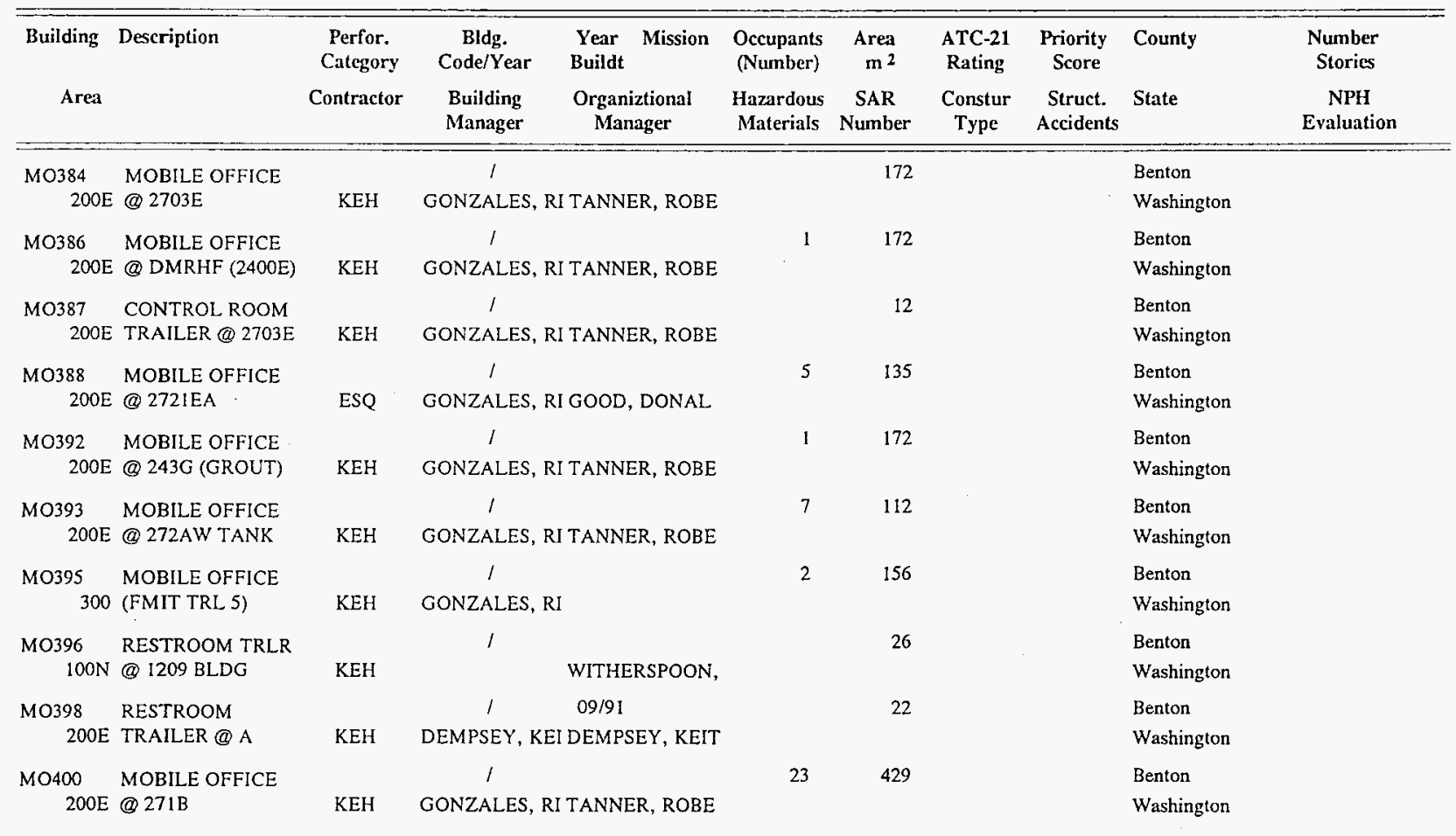


WHC.SD-SQA-CSWD-501 ReV 0

\begin{tabular}{|c|c|c|c|c|c|c|c|c|c|c|}
\hline Building & Description & $\begin{array}{c}\text { Perfor. } \\
\text { Category } \\
\text { Contractor }\end{array}$ & $\begin{array}{l}\text { Bldg. } \\
\text { Code/Year } \\
\text { Building } \\
\text { Manager }\end{array}$ & $\begin{array}{l}\text { Year Mission } \\
\text { Buildt } \\
\text { Organiztional } \\
\text { Manager }\end{array}$ & $\begin{array}{l}\text { Occupants } \\
\text { (Number) } \\
\text { Hazardous } \\
\text { Materials }\end{array}$ & $\begin{array}{c}\begin{array}{c}\text { Area } \\
\mathrm{m}^{2} \\
\text { SAR } \\
\text { Number }\end{array} \\
\text { S }\end{array}$ & $\begin{array}{c}\text { ATC-21 } \\
\text { Rating } \\
\text { Constur } \\
\text { Type }\end{array}$ & $\begin{array}{l}\text { Priority } \\
\text { Score } \\
\text { Struct. } \\
\text { Accidents }\end{array}$ & State & $\begin{array}{c}\text { Number } \\
\text { Stories } \\
\text { NPH } \\
\text { Evaluation }\end{array}$ \\
\hline $\begin{array}{r}\text { MO402 } \\
100 \mathrm{~K}\end{array}$ & $\begin{array}{l}\text { MOBILE OFFICE } \\
\text { AKA:1718K (FKA: }\end{array}$ & SNF & $\begin{array}{c}/ \\
\text { SCHMIDT, Jo }\end{array}$ & $\begin{array}{l}\text { 03/83 } \\
\text { SIDDOWAY, DE }\end{array}$ & 59 & 1018 & & & $\begin{array}{l}\text { Benton } \\
\text { Washington }\end{array}$ & \\
\hline $\begin{array}{l}\text { MO404 } \\
1100\end{array}$ & $\begin{array}{l}\text { MOBILE OFFICE } \\
\text { (10) } 1163\end{array}$ & KEH & $\begin{array}{c}/ \\
\text { GONZALES, R }\end{array}$ & $\begin{array}{l}01 / 84 \\
\text { I TANNER, ROBE }\end{array}$ & 36 & 687 & & & $\begin{array}{l}\text { Benton } \\
\text { Washington }\end{array}$ & \\
\hline $\begin{array}{l}\text { MO405 } \\
200 \mathrm{E}\end{array}$ & $\begin{array}{l}\text { MOBILE OFFICE } \\
\text { NW OF } 202 \mathrm{~A}\end{array}$ & TRP & BAILEY, RON & HAMRICK, DOU & 20 & 1288 & & & $\begin{array}{l}\text { Benton } \\
\text { Washington }\end{array}$ & \\
\hline $\begin{array}{l}\text { MO406 } \\
200 \mathrm{~W}\end{array}$ & $\begin{array}{l}\text { CHANGE/LUNCHR } \\
\text { OOM TRAILER @ }\end{array}$ & KEH & $\begin{array}{c}/ \\
\text { GONZALES, R }\end{array}$ & I TANNER, ROBE & 15 & 258 & & & $\begin{array}{l}\text { Benton } \\
\text { Washington }\end{array}$ & \\
\hline $\begin{array}{l}\text { MO407 } \\
200 E\end{array}$ & $\begin{array}{l}\text { MOBILE OFFICE } \\
\text { (1) } 2101 \mathrm{M}\end{array}$ & $\mathrm{KEH}$ & $\begin{array}{c}/ \\
\text { GONZALES, R }\end{array}$ & $\begin{array}{l}02 / 87 \\
\text { TANNER, ROBE }\end{array}$ & 16 & 429 & & & $\begin{array}{l}\text { Benton } \\
\text { Washington }\end{array}$ & \\
\hline $\begin{array}{l}\text { MO408 } \\
200 \mathrm{E}\end{array}$ & $\begin{array}{l}\text { MOBILE OFFICE } \\
@ 271 \mathrm{~B}\end{array}$ & KEH & $\begin{array}{c}\prime \\
\text { GONZALES, R }\end{array}$ & TANNER, ROBE & 12 & 258 & & & $\begin{array}{l}\text { Benton } \\
\text { Washington }\end{array}$ & \\
\hline $\begin{array}{l}\mathrm{MO409} \\
200 \mathrm{E}\end{array}$ & $\begin{array}{l}\text { ANALYTICAL } \\
\text { LAB TRAILER @ }\end{array}$ & TRP & $\begin{array}{c}l \\
\text { KAMMENZIN }\end{array}$ & & & 343 & & & $\begin{array}{l}\text { Benton } \\
\text { Washington }\end{array}$ & \\
\hline $\begin{array}{r}\text { MO410 } \\
200 \mathrm{E}\end{array}$ & $\begin{array}{l}\text { MOBILE OFFICE } \\
\text { ( } 271 \mathrm{~B}\end{array}$ & $\mathrm{KEH}$ & $\begin{array}{c}\text { I } \\
\text { GONZALES, RI }\end{array}$ & TANNER, ROBE & 14 & 258 & & & $\begin{array}{l}\text { Benton } \\
\text { Washington }\end{array}$ & \\
\hline $\begin{array}{l}\text { MO412 } \\
200 \mathrm{~W}\end{array}$ & $\begin{array}{l}\text { MASK CLEANING } \\
\text { STATION @2724W }\end{array}$ & $\mathrm{KEH}$ & $\begin{array}{c}\text { / } \\
\text { GONZALES, RI }\end{array}$ & TANNER, ROBE & 21 & 515 & & & $\begin{array}{l}\text { Benton } \\
\text { Washington }\end{array}$ & \\
\hline $\begin{array}{l}\mathrm{MO413} \\
200 \mathrm{E}\end{array}$ & $\begin{array}{l}\text { MOBILE OFFICE } \\
\text { @ } 2750 \mathrm{E}\end{array}$ & $\mathrm{KEH}$ & $\begin{array}{c}\prime \\
\text { GONZALES, RI }\end{array}$ & TANNER, ROBE & 12 & 687 & & & $\begin{array}{l}\text { Benton } \\
\text { Washington }\end{array}$ & \\
\hline
\end{tabular}


WHC-SD-SQA-CSWD-501 Rev 0

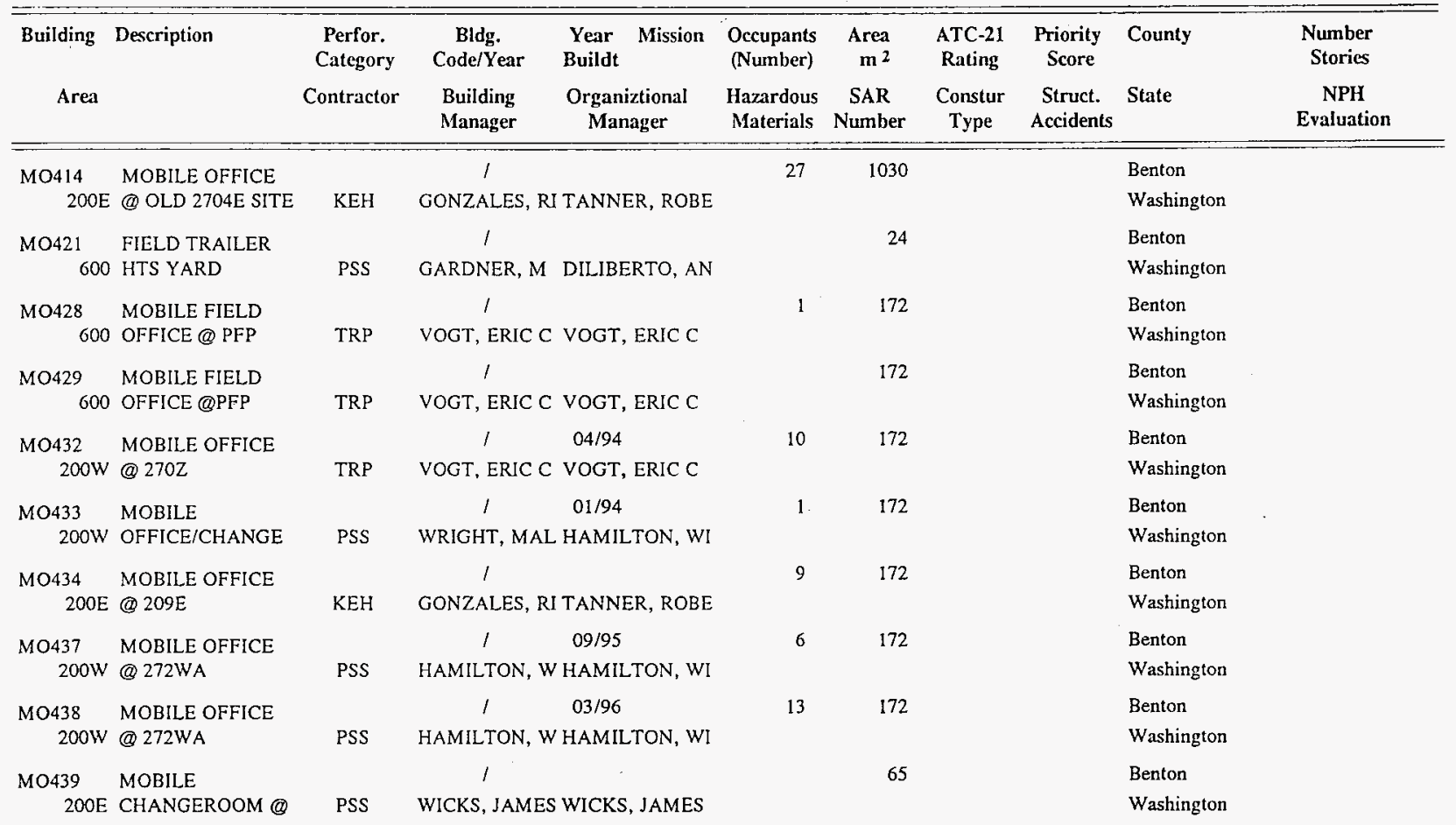


WHC-SD-SQA-CSWD-501 Rev 0

\begin{tabular}{|c|c|c|c|c|c|c|c|c|c|c|}
\hline Building & Description & $\begin{array}{c}\text { Perfor. } \\
\text { Category } \\
\text { Contractor }\end{array}$ & $\begin{array}{c}\text { Bldg. } \\
\text { Code/Year } \\
\text { Building } \\
\text { Manager }\end{array}$ & $\begin{array}{l}\text { Year Mission } \\
\text { Buildt } \\
\text { Organiztional } \\
\text { Manager }\end{array}$ & $\begin{array}{c}\text { Occupants } \\
\text { (Number) } \\
\text { Hazardous } \\
\text { Materials }\end{array}$ & $\begin{array}{c}\begin{array}{c}\text { Area } \\
\mathrm{m}^{2} \\
\text { SAR } \\
\text { Number }\end{array} \\
\text { Num }\end{array}$ & $\begin{array}{c}\text { ATC-21 } \\
\text { Rating } \\
\text { Constur } \\
\text { Type }\end{array}$ & $\begin{array}{l}\text { Priority } \\
\text { Score } \\
\text { Struct. } \\
\text { Accidents }\end{array}$ & State & $\begin{array}{c}\text { Number } \\
\text { Storics } \\
\text { NPH } \\
\text { Evaluation }\end{array}$ \\
\hline $\begin{array}{l}\text { MO441 } \\
200 \mathrm{~W}\end{array}$ & $\begin{array}{l}\text { MOBILE } \\
\text { CHANGEROOM }\end{array}$ & KEH & 1 & DEMPSEY, KEIT & & 15 & & & $\begin{array}{l}\text { Benton } \\
\text { Washington }\end{array}$ & \\
\hline $\begin{array}{l}\mathrm{MO} 442 \\
100 \mathrm{~K}\end{array}$ & $\begin{array}{l}\text { MOBILE OFFICE } \\
\text { (G) } 1726 \mathrm{~K}\end{array}$ & SNF & $\begin{array}{c}\text { / } \\
\text { BLEHM, LORI }\end{array}$ & SIDDOWAY, DE & 2 & 172 & & & $\begin{array}{l}\text { Benton } \\
\text { Washington }\end{array}$ & \\
\hline $\begin{array}{r}\text { MO443 } \\
300\end{array}$ & MOBILE OFFICE & PSS & $\begin{array}{c}/ \\
\text { ROBERTS, LEE }\end{array}$ & $\begin{array}{c}\quad 07 / 94 \\
\text { E DILIBERTO, AN }\end{array}$ & 2 & 51 & & & $\begin{array}{l}\text { Benton } \\
\text { Washington }\end{array}$ & \\
\hline $\begin{array}{l}\text { MO444 } \\
200 \mathrm{~W}\end{array}$ & $\begin{array}{l}\text { MOBILE OFFICE } \\
\text { ( } 3 \text { 23RD \& }\end{array}$ & KEH & $\begin{array}{c}\text { I } \\
\text { WITHERSPOO }\end{array}$ & WITHERSPOON, & & 53 & & & $\begin{array}{l}\text { Benton } \\
\text { Washington }\end{array}$ & \\
\hline $\begin{array}{l}\text { MO445 } \\
200 \mathrm{~W}\end{array}$ & $\begin{array}{l}\text { MOBILE OFFICE } \\
@ 23 R D \&\end{array}$ & $\mathrm{KEH}$ & $\begin{array}{c}\text { / } \\
\text { WITHERSPOO }\end{array}$ & & 3 & 53 & & & $\begin{array}{l}\text { Benton } \\
\text { Washington }\end{array}$ & \\
\hline $\begin{array}{l}\text { MO446 } \\
200 \mathrm{~W}\end{array}$ & $\begin{array}{l}\text { MOBILE OFFICE } \\
@ 23 \text { RD/DAYTON }\end{array}$ & $\mathrm{KEH}$ & $\begin{array}{c}\text { / } \\
\text { WITHERSPOO }\end{array}$ & WITHERSPOON, & 5 & 62 & & & $\begin{array}{l}\text { Benton } \\
\text { Washington }\end{array}$ & \\
\hline $\begin{array}{l}\text { MO447 } \\
200 \mathrm{~W}\end{array}$ & $\begin{array}{l}\text { STORAGE } \\
\text { TRAILER E/O }\end{array}$ & TWR & $\begin{array}{c}\text { ' } \\
\text { REBER, DAVI }\end{array}$ & WICKS, JAMES & & 9 & & & $\begin{array}{l}\text { Benton } \\
\text { Washington }\end{array}$ & \\
\hline $\begin{array}{l}\text { MO450 } \\
200 \mathrm{~W}\end{array}$ & $\begin{array}{l}\text { STORAGE } \\
\text { MOBILE N/O }\end{array}$ & TWR & 1 & WICKS, JAMES & & 9 & & & $\begin{array}{l}\text { Benton } \\
\text { Washington }\end{array}$ & \\
\hline $\begin{array}{l}\text { MO451 } \\
200 \mathrm{~W}\end{array}$ & $\begin{array}{l}\text { STORAGE } \\
\text { MOBILE NE/O }\end{array}$ & TWR & 1 & WICKS, JAMES & & 9 & & & $\begin{array}{l}\text { Benton } \\
\text { Washington }\end{array}$ & \\
\hline $\begin{array}{l}\mathrm{MO} 454 \\
\quad 200 \mathrm{~W}\end{array}$ & MOBILE OFFICE & KEH & $\begin{array}{c}l \\
\text { EGGEBRATEN }\end{array}$ & FOUCAULT, DA & 2 & 62 & & & $\begin{array}{l}\text { Benton } \\
\text { Washington }\end{array}$ & \\
\hline
\end{tabular}


WHC-SD-SQA-CSWD-501 Rev 0

\begin{tabular}{|c|c|c|c|c|c|c|c|c|c|c|}
\hline Area & Description & $\begin{array}{c}\text { Perfor. } \\
\text { Category } \\
\text { Contractor }\end{array}$ & $\begin{array}{l}\text { Bldg. } \\
\text { Code/Year } \\
\text { Building } \\
\text { Manager }\end{array}$ & $\begin{array}{l}\text { Year Mission } \\
\text { Buildt } \\
\text { Organiztional } \\
\text { Manager }\end{array}$ & $\begin{array}{c}\text { Occupants } \\
\text { (Number) } \\
\text { Hazardous } \\
\text { Materials }\end{array}$ & $\begin{array}{c}\text { Area } \\
\mathbf{m}^{2} \\
\text { SAR } \\
\text { Number }\end{array}$ & $\begin{array}{l}\text { ATC-21 } \\
\text { Rating } \\
\text { Constur } \\
\text { Type }\end{array}$ & $\begin{array}{l}\text { Priority } \\
\text { Score } \\
\text { Struct. } \\
\text { Accidents }\end{array}$ & State & $\begin{array}{c}\text { Number } \\
\text { Stories } \\
\text { NPH } \\
\text { Evaluation }\end{array}$ \\
\hline $\begin{array}{l}\text { MO461 } \\
200 \mathrm{~W}\end{array}$ & $\begin{array}{l}\text { INSTRUMENT } \\
\text { MOBILE @ 101SY }\end{array}$ & TWR & 1 & WICKS, JAMES & & 46 & & & $\begin{array}{l}\text { Benton } \\
\text { Washington }\end{array}$ & \\
\hline $\begin{array}{l}\text { M0465 } \\
200 \mathrm{~W}\end{array}$ & $\begin{array}{l}\text { MOBILE OFFICE } \\
@ \text { WRAP SITE }\end{array}$ & $\mathrm{KEH}$ & $\begin{array}{c}\prime \\
\text { HARRIS, MAR }\end{array}$ & FOUCAULT, DA & 4 & & & & $\begin{array}{l}\text { Benton } \\
\text { Washington }\end{array}$ & \\
\hline $\begin{array}{l}\text { MO535 } \\
200 \mathrm{~W}\end{array}$ & $\begin{array}{l}\text { MOBILE OFFICE } \\
\text { @ 272WA }\end{array}$ & PSS & $\begin{array}{c}/ \\
\text { HAMILTON, w }\end{array}$ & HAMILTON, WI & 3 & 120 & . & & $\begin{array}{l}\text { Benton } \\
\text { Washington }\end{array}$ & \\
\hline $\begin{array}{r}\text { MO536 } \\
600\end{array}$ & $\begin{array}{l}\text { SPECIAL } \\
\text { WAREHOUSE @ }\end{array}$ & KEH & $\begin{array}{c}\prime \\
\text { GONZALES, RI }\end{array}$ & I WALTON, CRAI & & 120 & & & $\begin{array}{l}\text { Benton } \\
\text { Washington }\end{array}$ & \\
\hline $\begin{array}{l}\text { MO537 } \\
200 E\end{array}$ & $\begin{array}{l}\text { CHANGE } \\
\text { TRAILER } @ 221 \mathrm{~B}\end{array}$ & TRP & $\frac{\prime}{\text { MIDGETT, JOE }}$ & MIDGETT, JOE & & 39 & & & $\begin{array}{l}\text { Benton } \\
\text { Washington }\end{array}$ & \\
\hline $\begin{array}{r}\text { MO539 } \\
.3000\end{array}$ & $\begin{array}{l}\text { MEN·S } \\
\text { RESTROOM }\end{array}$ & $\mathrm{KEH}$ & $\begin{array}{c}\prime \\
\text { HOLLADAY, J }\end{array}$ & TANNER, ROBE & & 29 & & & $\begin{array}{l}\text { Benton } \\
\text { Washington }\end{array}$ & \\
\hline $\begin{array}{l}\text { MO540 } \\
200 E\end{array}$ & $\begin{array}{l}\text { PORTABLE } \\
\text { CHANGE }\end{array}$ & TRP & $\begin{array}{c}\prime \\
\text { HAMRICK, DO }\end{array}$ & HAMRICK, DOU & & 15 & & & $\begin{array}{l}\text { Benton } \\
\text { Washington }\end{array}$ & \\
\hline $\begin{array}{l}\mathrm{MO5} 42 \\
200 \mathrm{E}\end{array}$ & $\begin{array}{l}\text { MOBILE OFFICE } \\
\text { @ 202A (ATT'D/ID }\end{array}$ & KEH & $\begin{array}{c}/ \\
\text { GONZALES, RI }\end{array}$ & TANNER, ROBE & & 120 & & & $\begin{array}{l}\text { Benton } \\
\text { Washington }\end{array}$ & \\
\hline $\begin{array}{r}\text { MO543 } \\
300\end{array}$ & $\begin{array}{l}\text { MOBILE } \\
\text { CHANGEROOM }\end{array}$ & $\mathrm{KEH}$ & $\frac{l}{\text { RITTENHOUSE }}$ & RITTENHOUSE, & 1 & 120 & & & $\begin{array}{l}\text { Benton } \\
\text { Washington }\end{array}$ & \\
\hline $\begin{array}{l}\text { MO549 } \\
100 \mathrm{~N}\end{array}$ & $\begin{array}{l}\text { LUNCH ROOM - } \\
\text { AKA TRL. } 1 \text { (ATT' }\end{array}$ & KEH & $\begin{array}{c}l \\
\text { STAIR, JEFFRE }\end{array}$ & WITHERSPOON, & 14 & 167 & & & $\begin{array}{l}\text { Benton } \\
\text { Washington }\end{array}$ & \\
\hline
\end{tabular}


WHC-SD-SQA-CSWD-501 ReV D

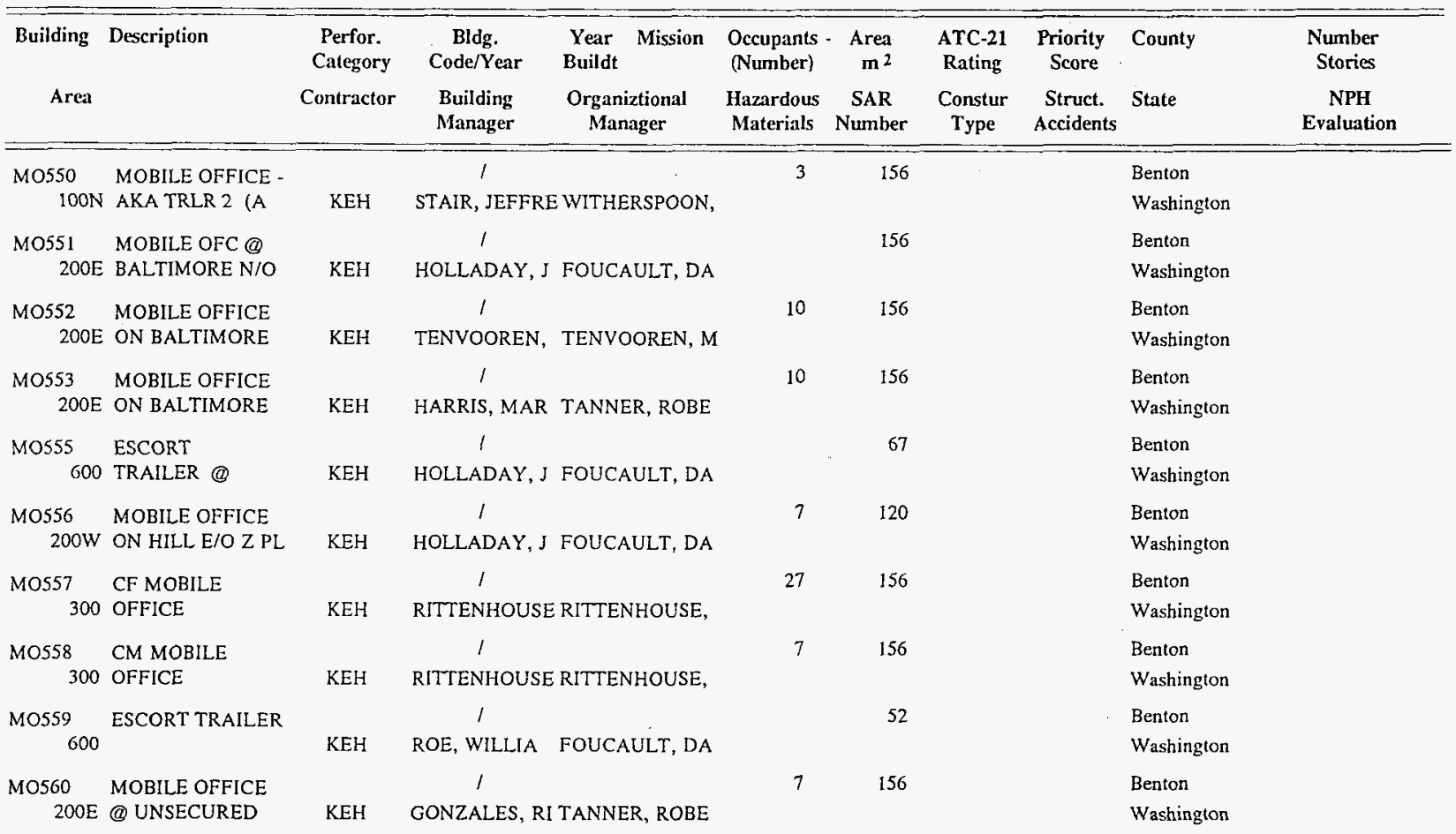


WHC-SD-SQA-CSWD-501 Rev 0

\begin{tabular}{|c|c|c|c|c|c|c|c|c|c|c|}
\hline Building & Description & $\begin{array}{l}\text { Perfor. } \\
\text { Category }\end{array}$ & $\begin{array}{c}\text { Bldg. } \\
\text { Code/Year }\end{array}$ & $\underset{\text { Year Mission }}{\text { Buildt }}$ & $\begin{array}{l}\text { Occupants } \\
\text { (Number) }\end{array}$ & $\begin{array}{r}\text { Area } \\
\mathrm{m}^{2}\end{array}$ & $\begin{array}{l}\text { ATC-21 } \\
\text { Rating }\end{array}$ & $\begin{array}{c}\text { Priority } \\
\text { Score }\end{array}$ & County & $\begin{array}{c}\text { Number } \\
\text { Stories }\end{array}$ \\
\hline Area & & Contractor & $\begin{array}{l}\text { Building } \\
\text { Manager }\end{array}$ & $\begin{array}{l}\text { Organiztional } \\
\text { Manager }\end{array}$ & $\begin{array}{c}\text { Hazardous } \\
\text { Materials }\end{array}$ & $\begin{array}{c}\text { SAR } \\
\text { Number }\end{array}$ & $\begin{array}{l}\text { Constur } \\
\text { Type }\end{array}$ & $\begin{array}{l}\text { Struct. } \\
\text { Accidents }\end{array}$ & State & $\begin{array}{c}\text { NPH } \\
\text { Evaluation }\end{array}$ \\
\hline MO565 & & & 1 & & & & & & Benton & \\
\hline 300 & & $\mathrm{KEH}$ & & & & & & & Washington & \\
\hline MO569 & RESTROOM & & 1 & & & 22 & & & Benton & \\
\hline $200 E$ & TRAILER@244CR & $\mathrm{KEH}$ & DEMPSEY, KEI & I DEMPSEY, KEIT & & & & & Washington & \\
\hline MO570 & MOBILE OFFICE & & 1 & & 52 & 167 & & & Benton & \\
\hline $200 \mathrm{E}$ & Q $244 \mathrm{CR}$ & $\mathrm{KEH}$ & DEMPSEY, KEI & I DEMPSEY, KEIT & & & & & Washington & \\
\hline MO57I & OFFICE/LUNCHR & & 1 & & & 67 & & & Benton & \\
\hline $200 \mathrm{E}$ & OOM TRLR@ & $\mathrm{KEH}$ & DEMPSEY, KEI & I DEMPSEY, KEIT & & & & & Washington & \\
\hline MO573 & CHANGE/LUNCHR & & I & & & 18 & & & Benton & \\
\hline $200 E$ & OOM TRLR X FM & $\mathrm{KEH}$ & WITHERSPOO & WITHERSPOON, & & & & & Washington & \\
\hline $\mathrm{MO574}$ & CHANGEROOM & & 1 & & & 22 & & & Benton & \\
\hline 3000 & TRAILER@224B & KEH & DEMPSEY, KEI & I DEMPSEY, KEIT & & & & & Washington & \\
\hline MO656 & MOBILE OFFICE & & $r$ & & & 9 & & & Benton & \\
\hline 300 & & KEH & & TANNER, ROBE & & & & & Washington & \\
\hline $\mathrm{MO662}$ & FIELD MOBILE@ & & 1 & & & 48 & & & Benton & \\
\hline 600 & SLAB YARD & $\mathrm{KEH}$ & HOLLADAY, J & FOUCAULT, DA & & & & & Washington & \\
\hline MO666 & MOBILE OFFICE & & 1 & & 2 & 48 & & & Benton & \\
\hline $200 E$ & (g) $202 \mathrm{~A}$ & TRP & KAMMENZIN & HAMRICK, DOU & & & & & Washington & \\
\hline MO667 & FIELD & & 1 & & & 22 & & & Benton & \\
\hline 600 & LABORATORY@ & $\mathrm{KEH}$ & & DEMPSEY, KEIT & & & & & Washington & \\
\hline
\end{tabular}


WHC-SD-SQA-CSWD-501 Rev 0

\begin{tabular}{|c|c|c|c|c|c|c|c|c|c|c|}
\hline Building & Description & $\begin{array}{c}\text { Perfor. } \\
\text { Category } \\
\text { Contractor }\end{array}$ & $\begin{array}{c}\text { Bldg. } \\
\text { Code/Year } \\
\text { Building } \\
\text { Manager }\end{array}$ & $\begin{array}{l}\text { Year Mission } \\
\text { Buildt } \\
\text { Organiztional } \\
\text { Manager }\end{array}$ & $\begin{array}{l}\text { Occupants } \\
\text { (Number) } \\
\text { Hazardous } \\
\text { Materials }\end{array}$ & $\begin{array}{c}\text { Area } \\
\mathrm{m}^{2} \\
\text { SAR } \\
\text { Number }\end{array}$ & $\begin{array}{c}\text { ATC-21 } \\
\text { Rating } \\
\text { Constur } \\
\text { Type }\end{array}$ & $\begin{array}{l}\text { Priority } \\
\text { Score } \\
\text { Struct. } \\
\text { Accidents }\end{array}$ & State & $\begin{array}{l}\text { Number } \\
\text { Stories } \\
\text { NPH } \\
\text { Evaluation }\end{array}$ \\
\hline $\begin{array}{r}\text { MO674 } \\
200 \mathrm{E}\end{array}$ & $\begin{array}{l}\text { MOBILE OFFICE } \\
\text { @ } 243 \mathrm{G} \text { (GROUT) }\end{array}$ & $\mathrm{KEH}$ & $\begin{array}{c}/ \\
\text { GONZALES, RI }\end{array}$ & I TANNER, ROBE & & 48 & & & $\begin{array}{l}\text { Benton } \\
\text { Washington }\end{array}$ & \\
\hline $\begin{array}{r}\mathrm{MO} 02 \mathrm{2} \\
600\end{array}$ & $\begin{array}{l}\text { MOBILE OFFICE } \\
@ \text { HTS PIPEYARD }\end{array}$ & PSS & $\frac{\prime}{\text { GARDNER, M }}$ & DILIBERTO, AN & & 48 & & & $\begin{array}{l}\text { Benton } \\
\text { Washington }\end{array}$ & \\
\hline $\begin{array}{l}\text { MO707 } \\
200 \mathrm{E}\end{array}$ & $\begin{array}{l}\text { PATROL } \\
\text { TRAILER @202A }\end{array}$ & TRP & $\frac{\text { / }}{\text { KAMMENZIN }}$ & HAMRICK, DOU & 4 & 48 & & & $\begin{array}{l}\text { Benton } \\
\text { Washington }\end{array}$ & \\
\hline $\begin{array}{l}\text { MO708 } \\
\quad 200 \mathrm{~W}\end{array}$ & $\begin{array}{l}\text { MOBILE OFFICE } \\
\text { (G) } 201 \mathrm{R}\end{array}$ & TWR & $\begin{array}{c}1 \\
\text { GONZALES, RI }\end{array}$ & I ERMOLD, LEON & & 48 & & & $\begin{array}{l}\text { Benton } \\
\text { Washington }\end{array}$ & \\
\hline $\begin{array}{r}\text { MO709 } \\
300\end{array}$ & $\begin{array}{l}\text { MOBILE OFFICE } \\
\text { ALSO KNOWN AS }\end{array}$ & STS & $\begin{array}{c}\prime \\
\text { HUNTER, JAM }\end{array}$ & BROWN, LARRY & & 39 & & & $\begin{array}{l}\text { Benton } \\
\text { Washington }\end{array}$ & \\
\hline $\begin{array}{l}\text { MO712 } \\
\quad 200 \mathrm{~W}\end{array}$ & $\begin{array}{l}\text { MOBILE OFFICE } \\
\text { S/O } 622 \mathrm{G}\end{array}$ & $\mathrm{KEH}$ & $\begin{array}{c}\prime \\
\text { GONZALES, RI }\end{array}$ & I DILIBERTO, AN & & 39 & & & $\begin{array}{l}\text { Benton } \\
\text { Washington }\end{array}$ & \\
\hline $\begin{array}{l}\text { M0713 } \\
600\end{array}$ & $\begin{array}{l}\text { PORTABLE } \\
\text { GEN/WATER }\end{array}$ & $\mathrm{KEH}$ & $\begin{array}{c}1 \\
\text { GONZALES, RI }\end{array}$ & I TANNER, ROBE & & 48 & & & $\begin{array}{l}\text { Benton } \\
\text { Washington }\end{array}$ & \\
\hline $\begin{array}{l}\text { MO7!6 } \\
\quad 200 \mathrm{~W}\end{array}$ & $\begin{array}{l}\text { STORAGE } \\
\text { TRAILER ON }\end{array}$ & $\mathrm{KEH}$ & $\begin{array}{c}\prime \\
\text { HOLLADAY, }\rfloor\end{array}$ & FOUCAULT, DA & & 67 & & & $\begin{array}{l}\text { Benton } \\
\text { Washington }\end{array}$ & \\
\hline $\begin{array}{l}\text { MO717 } \\
200 E\end{array}$ & $\begin{array}{l}\text { LUNCHROOM } \\
\text { TRAILER@SLAB }\end{array}$ & $\mathrm{KEH}$ & $\begin{array}{c}\text { / } \\
\text { WITHERSPOO }\end{array}$ & WITHERSPOON, & & 10 & & & $\begin{array}{l}\text { Benton } \\
\text { Washington }\end{array}$ & \\
\hline $\begin{array}{l}\text { MO718 } \\
200 \mathrm{E}\end{array}$ & $\begin{array}{l}\text { LUNCHROOM } \\
\text { TRAILER @ SLAB }\end{array}$ & KEH & $\begin{array}{c}l \\
\text { WITHERSPOO }\end{array}$ & WITHERSPOON, & & 10 & & & $\begin{array}{l}\text { Benton } \\
\text { Washington }\end{array}$ & \\
\hline
\end{tabular}


WHC-SD-SQA-CSWD-501 Rev 0

\begin{tabular}{|c|c|c|c|c|c|c|c|c|c|c|}
\hline Building & Description & $\begin{array}{c}\text { Perfor. } \\
\text { Category }\end{array}$ & $\begin{array}{c}\text { Bldg. } \\
\text { Code/Year }\end{array}$ & $\begin{array}{l}\text { Year Mission } \\
\text { Buildt }\end{array}$ & $\begin{array}{l}\text { Occupants } \\
\text { (Number) }\end{array}$ & $\begin{array}{r}\text { Area } \\
\mathrm{m}^{2}\end{array}$ & $\begin{array}{l}\text { ATC-21 } \\
\text { Rating }\end{array}$ & $\begin{array}{c}\text { Priority } \\
\text { Score }\end{array}$ & County & $\begin{array}{l}\text { Number } \\
\text { Stories }\end{array}$ \\
\hline Area & & Contractor & $\begin{array}{l}\text { Building } \\
\text { Manager }\end{array}$ & $\begin{array}{l}\text { Organiztional } \\
\text { Manager }\end{array}$ & $\begin{array}{l}\text { Hazardous } \\
\text { Materials }\end{array}$ & $\begin{array}{c}\text { SAR } \\
\text { Number }\end{array}$ & $\begin{array}{l}\text { Constur } \\
\text { Type }\end{array}$ & $\begin{array}{l}\text { Struct. } \\
\text { Accidents }\end{array}$ & State & $\begin{array}{c}\text { NPH } \\
\text { Evaluation }\end{array}$ \\
\hline MO719 & CALIBRATION & & 1 & $08 / 81$ & & 10 & & & Benton & \\
\hline $200 \mathrm{~W}$ & LAB @ $272 W$ & TWR & BROWN, ROD & TANNER, ROBE & & & & & Washington & \\
\hline $\mathrm{MO} 720$ & MOBILE OFFICE & & 1 & & 105 & 1374 & & & Benton & \\
\hline $200 \mathrm{~W}$ & @ 272WA & PSS & HAMILTON, W & HAMILTON, WI & & & & & Washington & \\
\hline MO721 & MOBILE CHANGE & & 1 & & 35 & 343 & & & Benton & \\
\hline $200 \mathrm{~W}$ & FACILITY@ & PSS & HAMILTON, W & HAMILTON, WI & & & & & Washington & \\
\hline MO722 & MOBILE OFFICE & & 1 & & & 172 & & & Benton & \\
\hline $200 \mathrm{~W}$ & (a) $224 \mathrm{U}$ & $\mathrm{KEH}$ & GONZALES, RI & I TANNER, ROBE & & & & & Washington & \\
\hline MO723 & MOBILE OFFICE & & 1 & & & 172 & & & Benton & \\
\hline 600 & (Q) GATE $813,200 E$ & TWR & & ERMOLD, LEON & & & & & Washington & \\
\hline MO724 & MOBILE OFFICE & & 1 & & 5 & 172 & & & Benton & \\
\hline 600 & $@$ GATE 813,200E & PSS & CARTER, STE & KELLY, JAMES & & & & & Washington & \\
\hline MO725 & MOBILE OFFICE & & 1 & & 9 & 172 & & & Benton & \\
\hline 600 & @ GATE $813,200 \mathrm{E}$ & PSS & CARTER, STE & KELLY, JAMES & & & & & Washington & \\
\hline MO727 & MOBILE@LERF & & 1 & & & 36 & & & Benton & \\
\hline $200 \mathrm{E}$ & BASINS & TWR & GEARY, JAME & WICKS, JAMES & & & & & Washington & \\
\hline MO728 & CHANGE & & 1 & & & 67 & & & Benton & \\
\hline 600 & FACILITY@ & TWR & SAVINO, ANT & ERMOLD, LEON & & & . & & Washington & \\
\hline MO729 & RESTROOM & & 1 & & & 24 & & & Benton & \\
\hline 600 & FACILITY@ & TWR & SAVINO, ANT & ERMOLD, LEON & & & & & Washington & \\
\hline
\end{tabular}


WHC-SD-SQA-CSWD-501 Rev 0

\begin{tabular}{|c|c|c|c|c|c|c|c|c|c|c|}
\hline Building & Description & $\begin{array}{c}\text { Perfor. } \\
\text { Category } \\
\text { Contractor }\end{array}$ & $\begin{array}{c}\text { Bldg. } \\
\text { Code/Year } \\
\text { Building } \\
\text { Manager }\end{array}$ & $\begin{array}{l}\text { Year Mission } \\
\text { Buildt } \\
\text { Organiztional } \\
\text { Manager }\end{array}$ & $\begin{array}{c}\text { Occupants } \\
\text { (Number) } \\
\text { Hazardous } \\
\text { Materials }\end{array}$ & $\begin{array}{c}\text { Area } \\
\mathrm{m}^{2} \\
\text { SAR } \\
\text { Number }\end{array}$ & $\begin{array}{l}\text { ATC-21 } \\
\text { Rating } \\
\text { Constur } \\
\text { Type }\end{array}$ & $\begin{array}{l}\text { Priority } \\
\text { Score } \\
\text { Struct. } \\
\text { Accidents }\end{array}$ & State & $\begin{array}{c}\text { Number } \\
\text { Stories } \\
\text { NPH } \\
\text { Evaluation }\end{array}$ \\
\hline $\begin{array}{r}\text { MO730 } \\
600\end{array}$ & $\begin{array}{l}\text { RESTROOM } \\
\text { FACILITY @ }\end{array}$ & PSS & $\begin{array}{c}\text { / } \\
\text { FILIP, JEFFRE }\end{array}$ & KELLY, JAMES & & 60 & & & $\begin{array}{l}\text { Benton } \\
\text { Washington }\end{array}$ & \\
\hline $\begin{array}{r}\text { MO731 } \\
600\end{array}$ & $\begin{array}{l}\text { MOBILE OFFICE } \\
@ \text { GATE } 813\end{array}$ & TWR & $\begin{array}{c}\text { I } \\
\text { REBER, DAVI }\end{array}$ & WICKS, JAMES & & 146 & & & $\begin{array}{l}\text { Benton } \\
\text { Washington }\end{array}$ & \\
\hline $\begin{array}{r}\text { MO732 } \\
600\end{array}$ & $\begin{array}{l}\text { MOBILE OFFICE } \\
\text { (ं) HWVP SITE }\end{array}$ & TWR & $\begin{array}{c}\prime \\
\text { SHOWALTER, }\end{array}$ & WICKS, JAMES & 16 & 172 & & & $\begin{array}{l}\text { Benton } \\
\text { Washington }\end{array}$ & \\
\hline $\begin{array}{r}\text { MO733 } \\
600\end{array}$ & $\begin{array}{l}\text { FIELD OFFICE } \\
\text { MOBILE @ GATE }\end{array}$ & TWR & $\begin{array}{c}/ \\
\text { SHOWALTER, }\end{array}$ & WICKS, JAMES & 25 & 67 & & & $\begin{array}{l}\text { Benton } \\
\text { Washington }\end{array}$ & \\
\hline $\begin{array}{r}\text { MO734 } \\
600\end{array}$ & $\begin{array}{l}\text { MOBILE OFFICE } \\
@ \text { HWVP (GATE }\end{array}$ & TWR & $\begin{array}{c}\text { I } \\
\text { SHOWALTER, }\end{array}$ & WICKS, JAMES & 13 & 172 & & & $\begin{array}{l}\text { Benton } \\
\text { Washington }\end{array}$ & \\
\hline $\begin{array}{l}\text { MO738 } \\
200 \mathrm{~W}\end{array}$ & $\begin{array}{l}\text { TANK FARM } \\
\text { MOBILE FIELD }\end{array}$ & PSS & $\begin{array}{c}\text { I } \\
\text { HAMILTON, W }\end{array}$ & V HAMILTON, WI & & 48 & & & $\begin{array}{l}\text { Benton } \\
\text { Washington }\end{array}$ & \\
\hline $\begin{array}{l}\text { MO739 } \\
200 \mathrm{~W}\end{array}$ & $\begin{array}{l}\text { SWP CHANGE } \\
\text { TRAILER @ 2706T }\end{array}$ & PSS & $\begin{array}{c}\text { I } \\
\text { HAMILTON, W }\end{array}$ & $\checkmark$ HAMILTON, WI & & 48 & & & $\begin{array}{l}\text { Benton } \\
\text { Washington }\end{array}$ & \\
\hline $\begin{array}{r}\text { MO741 } \\
300\end{array}$ & HPT OFFICE@340 & PSS & $\begin{array}{c}/ \\
\text { ROBERTS, LEE }\end{array}$ & $\begin{array}{c}06 / 94 \\
\text { E DILIBERTO, AN }\end{array}$ & I & 22 & & & $\begin{array}{l}\text { Benton } \\
\text { Washington }\end{array}$ & \\
\hline $\begin{array}{r}\text { MO742 } \\
600\end{array}$ & & TWR & 1 & ERMOLD, LEON & & & & & $\begin{array}{l}\text { Benton } \\
\text { Washington }\end{array}$ & \\
\hline $\begin{array}{l}\text { MO743 } \\
200 \mathrm{~W}\end{array}$ & $\begin{array}{l}\text { MOBILE } \\
\text { SHOP/OFFICE @ }\end{array}$ & PSS & $\begin{array}{c}\text { I } \\
\text { HAMILTON, W }\end{array}$ & $\begin{array}{c}07 / 94 \\
\text { VHAMILTON, WI }\end{array}$ & 11 & 515 & & & $\begin{array}{l}\text { Benton } \\
\text { Washington }\end{array}$ & \\
\hline
\end{tabular}


WHC-SD-SQA-CSWD-501 Rev 0

\begin{tabular}{|c|c|c|c|c|c|c|c|c|c|c|}
\hline Building & Description & $\begin{array}{c}\text { Perfor. } \\
\text { Category } \\
\text { Contractor }\end{array}$ & $\begin{array}{l}\text { Bldg. } \\
\text { Code/Year } \\
\text { Building } \\
\text { Manager }\end{array}$ & $\begin{array}{l}\text { Year Mission } \\
\text { Buildt } \\
\text { Organiztional } \\
\text { Manager }\end{array}$ & $\begin{array}{c}\text { Occupants } \\
\text { (Number) } \\
\text { Hazardous } \\
\text { Materials }\end{array}$ & $\begin{array}{c}\text { Area } \\
\text { m }^{2} \\
\text { SAR } \\
\text { Number }\end{array}$ & $\begin{array}{c}\text { ATC-21 } \\
\text { Rating } \\
\text { Constur } \\
\text { Type }\end{array}$ & $\begin{array}{l}\text { Priority } \\
\text { Score } \\
\text { Struct. } \\
\text { Accidents }\end{array}$ & State & $\begin{array}{c}\text { Number } \\
\text { Stories } \\
\text { NPH } \\
\text { Evaluation }\end{array}$ \\
\hline $\begin{array}{r}\text { MO744 } \\
300\end{array}$ & $\begin{array}{l}\text { MOBILE OFFICE } \\
@ \text { TEDF }\end{array}$ & PSS & $\frac{l}{\text { ROBERTS, LEE }}$ & E DILIBERTO, AN & 11 & 172 & & & $\begin{array}{l}\text { Benton } \\
\text { Washington }\end{array}$ & \\
\hline $\begin{array}{r}\text { M0745 } \\
300\end{array}$ & $\begin{array}{l}\text { MOBILE OFFICE } \\
\text { (Q) TEDF }\end{array}$ & PSS & $\begin{array}{c}l \\
\text { ROBERTS, LEE }\end{array}$ & EILIBERTO, AN & 6 & 172 & & & $\begin{array}{l}\text { Benton } \\
\text { Washington }\end{array}$ & \\
\hline $\begin{array}{l}\text { MO815 } \\
200 E\end{array}$ & $\begin{array}{l}\text { CHANGE } \\
\text { TRAILER@ }\end{array}$ & TWR & $\begin{array}{c}\prime \\
\text { GEARY, JAME }\end{array}$ & $\begin{array}{l}10 / 91 \\
\text { WICKS, JAMES }\end{array}$ & & 36 & & & $\begin{array}{l}\text { Benton } \\
\text { Washington }\end{array}$ & \\
\hline $\begin{array}{l}\text { MO816 } \\
200 E\end{array}$ & $\begin{array}{l}\text { CHANGE } \\
\text { TRAILER@244A }\end{array}$ & TWR & $\begin{array}{c}l \\
\text { ROSS, WILLIA }\end{array}$ & $\begin{array}{l}10 / 91 \\
\text { WICKS, JAMES }\end{array}$ & & 36 & & & $\begin{array}{l}\text { Benton } \\
\text { Washington }\end{array}$ & \\
\hline $\begin{array}{l}\text { MO817 } \\
200 \mathrm{~W}\end{array}$ & $\begin{array}{l}\text { CHANGE } \\
\text { TRAILER@TX }\end{array}$ & TWR & $\begin{array}{c}\prime \\
\text { WICKS, JAMES }\end{array}$ & $\begin{array}{l}10 / 91 \\
\text { WICKS, JAMES }\end{array}$ & & 36 & & & $\begin{array}{l}\text { Benton } \\
\text { Washington }\end{array}$ & \\
\hline $\begin{array}{l}\text { MO818 } \\
200 E\end{array}$ & $\begin{array}{l}\text { CHANGE } \\
\text { TRAILER @ }\end{array}$ & TWR & $\begin{array}{c}\text { I } \\
\text { GEARY, JAME }\end{array}$ & $\begin{array}{l}10 / 91 \\
\text { WICKS, JAMES }\end{array}$ & & 36 & & & $\begin{array}{l}\text { Benton } \\
\text { Washington }\end{array}$ & \\
\hline $\begin{array}{l}\text { MO819 } \\
200 \mathrm{~W}\end{array}$ & $\begin{array}{l}\text { CHANGE } \\
\text { TRAILER @SX }\end{array}$ & TWR & WICKS, JAMES & $\begin{array}{c}10 / 91 \\
\text { WICKS, JAMES }\end{array}$ & & 36 & & & $\begin{array}{l}\text { Benton } \\
\text { Washington }\end{array}$ & \\
\hline $\begin{array}{l}\text { MO820 } \\
200 E\end{array}$ & $\begin{array}{l}\text { CHANGE } \\
\text { TRAILER (G) AN }\end{array}$ & TWR & $\begin{array}{c}\prime \\
\text { ROSS, WILLIA }\end{array}$ & $\begin{array}{l}10 / 91 \\
\text { WICKS, JAMES }\end{array}$ & & 36 & & & $\begin{array}{l}\text { Benton } \\
\text { Washington }\end{array}$ & \\
\hline $\begin{array}{l}\text { MO821 } \\
200 \mathrm{~W}\end{array}$ & $\begin{array}{l}\text { CHANGE } \\
\text { TRAILER @ 207T }\end{array}$ & TWR & $\frac{\prime}{\text { WICKS, JAMES }}$ & $\begin{array}{l}10 / 91 \\
\text { WICKS, JAMES }\end{array}$ & & 36 & & & $\begin{array}{l}\text { Benton } \\
\text { Washington }\end{array}$ & \\
\hline $\begin{array}{l}\text { MO822 } \\
200 E\end{array}$ & $\begin{array}{l}\text { CHANGE } \\
\text { TRAILER @241C }\end{array}$ & TWR & $\begin{array}{c}\text { ' } \\
\text { ROSS, WILLIA }\end{array}$ & $\begin{array}{l}10 / 91 \\
\text { WICKS, JAMES }\end{array}$ & & 36 & & & $\begin{array}{l}\text { Benton } \\
\text { Washington }\end{array}$ & \\
\hline
\end{tabular}


WHC-SD-SQA-CSWD-501 Rev 0

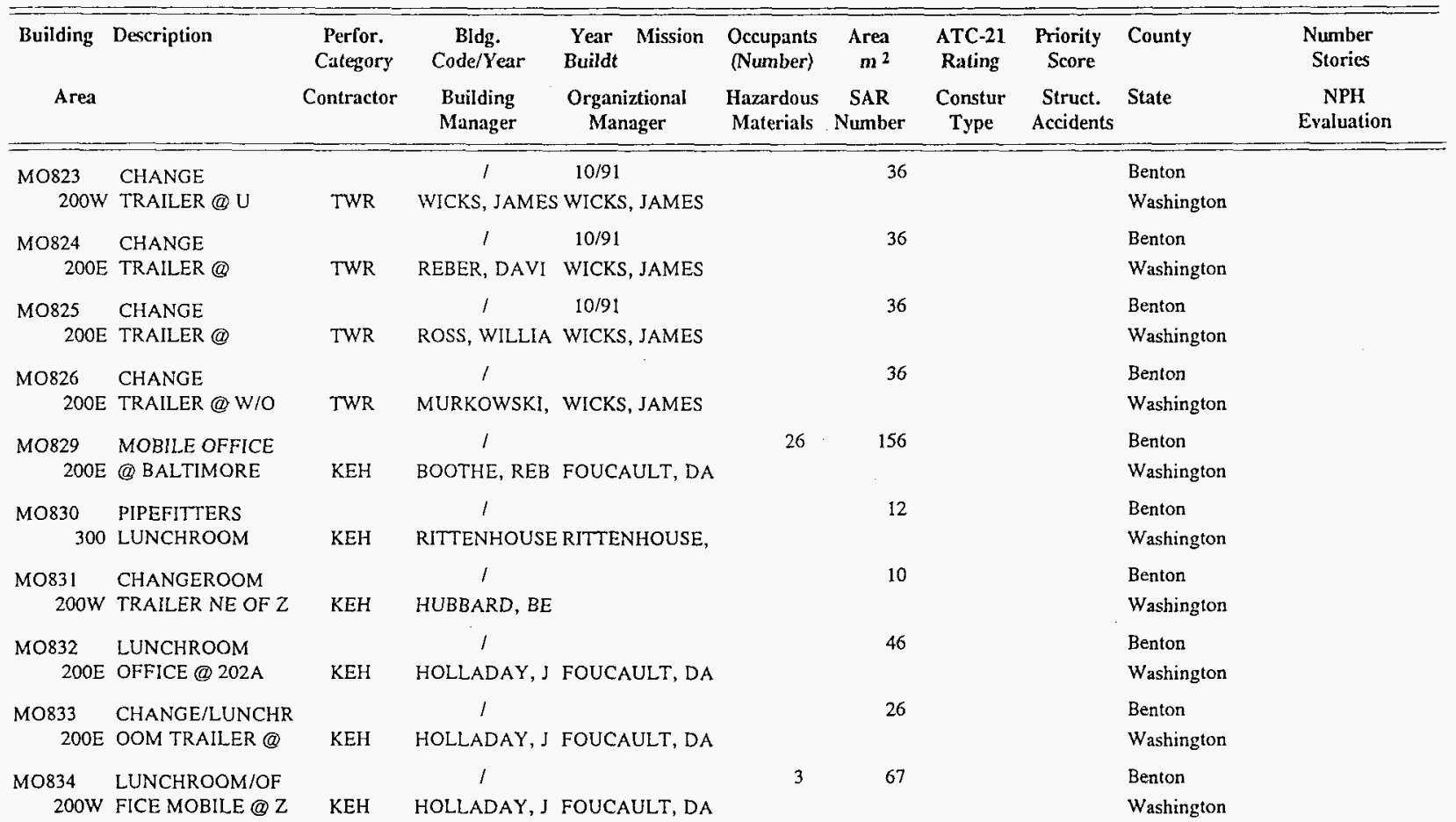


WHC-SD-SQA-CSWD-501 Rev 0

\begin{tabular}{|c|c|c|c|c|c|c|c|c|c|c|}
\hline Building & Description & $\begin{array}{l}\text { Perfor. } \\
\text { Category }\end{array}$ & $\begin{array}{c}\text { Bldg. } \\
\text { Code/Year }\end{array}$ & $\begin{array}{c}\text { Year Mission } \\
\text { Buildt }\end{array}$ & $\begin{array}{l}\text { Occupants } \\
\text { (Number) }\end{array}$ & $\begin{array}{r}\text { Area } \\
\text { m }^{2}\end{array}$ & $\begin{array}{l}\text { ATC-21 } \\
\text { Rating }\end{array}$ & $\begin{array}{l}\text { Priority } \\
\text { Score }\end{array}$ & County & $\begin{array}{l}\text { Number } \\
\text { Stories }\end{array}$ \\
\hline Area & & Contractor & $\begin{array}{l}\text { Building } \\
\text { Manager }\end{array}$ & $\begin{array}{l}\text { Organiztional } \\
\text { Manager }\end{array}$ & $\begin{array}{l}\text { Hazardous } \\
\text { Materials }\end{array}$ & $\begin{array}{c}\text { SAR } \\
\text { Number }\end{array}$ & $\begin{array}{l}\text { Constur } \\
\text { Type }\end{array}$ & $\begin{array}{l}\text { Struct. } \\
\text { Accidents }\end{array}$ & State & $\begin{array}{c}\text { NPH } \\
\text { Evaluation }\end{array}$ \\
\hline MO835 & LUNCH/OFFICE & & I & & & 67 & & & Benton & \\
\hline $200 E$ & MOBILE W/O $271 \mathrm{~T}$ & $\mathrm{KEH}$ & HOLLADAY, J & FOUCAULT, DA & & & & & Washington & \\
\hline MO836 & (DEMOLISHED) & & 1 & & & 13 & & & Benton & \\
\hline 300 & CHANGE & $\mathrm{KEH}$ & & RITTENHOUSE, & & & & & Washington & \\
\hline MO837 & LUNCHROOM & & 1 & & & 56 & & & Benton & \\
\hline $200 \mathrm{~W}$ & TRAILER ON & $\mathrm{KEH}$ & HOLLADAY, J & FOUCAULT, DA & & & & & Washington & \\
\hline MO838 & MOBILE OFFICE & & 1 & & & 112 & & & Benton & \\
\hline $200 E$ & (G) 4TH \& & $\mathrm{KEH}$ & GONZALES, RI & I TANNER, ROBE & & & & & Washington & \\
\hline MO839 & LUNCHROOM/OF & & 1 & & & 67 & & & Benton & \\
\hline $200 \mathrm{~W}$ & FICE MOBILE@Z & $\mathrm{KEH}$ & HOLLADAY, J & FOUCAULT, DA & & & & & Washington & \\
\hline MO840 & RECG \& DISTRIB. & & 1 & & & 67 & & & Benton & \\
\hline $200 E$ & OFC N/O 4TH ON & $\mathrm{KEH}$ & TENVOOREN, & TENVOOREN, M & & & & & Washington & \\
\hline MO841 & MOBILE OFFICE & & 1 & & 1 & 112 & & & Benton & \\
\hline $200 W$ & ON HILL E/O Z PL & $\mathrm{KEH}$ & HOLLADAY, J & FOUCAULT, DA & & & & & Washington & \\
\hline $\mathrm{MO} 842$ & ELECTRICIANS & & 1 & & & 67 & & & Benton & \\
\hline 300 & LUNCHROOM & $\mathrm{KEH}$ & RITTENHOUSE & RITTENHOUSE, & & & & & Washington & \\
\hline MO843 & STORAGE/SHOP & & 1 & & & 67 & & & Benton & \\
\hline $200 E$ & TRAILER @ 202A & $\mathrm{KEH}$ & HOLLADAY, J & FOUCAULT, DA & & & & & Washington & \\
\hline MO844 & OFFICE/LUNCHR & & 1 & & & 67 & & & Benton & \\
\hline $200 E$ & OOM TRAILER @ & $\mathrm{KEH}$ & HOLLADAY, J & FOUCAULT, DA & & & & & Washington & \\
\hline
\end{tabular}


WHC-SD-SQA-CSWD-501 ReV 0

\begin{tabular}{|c|c|c|c|c|c|c|c|c|c|c|}
\hline Building & Description & $\begin{array}{l}\text { Perfor. } \\
\text { Category }\end{array}$ & $\begin{array}{c}\text { Bldg. } \\
\text { Code/Year }\end{array}$ & $\begin{array}{l}\text { Year Mission } \\
\text { Buildt }\end{array}$ & $\begin{array}{l}\text { Occupants } \\
\text { (Number) }\end{array}$ & $\begin{array}{r}\text { Area } \\
\mathrm{m}^{2}\end{array}$ & $\begin{array}{l}\text { ATC-21 } \\
\text { Rating }\end{array}$ & $\begin{array}{c}\text { Priority } \\
\text { Score }\end{array}$ & County & $\begin{array}{l}\text { Number } \\
\text { Stories }\end{array}$ \\
\hline Area & & Contractor & $\begin{array}{l}\text { Building } \\
\text { Manager }\end{array}$ & $\begin{array}{l}\text { Organiztional } \\
\text { Manager }\end{array}$ & $\begin{array}{l}\text { Hazardous } \\
\text { Materials }\end{array}$ & $\begin{array}{c}\text { SAR } \\
\text { Number }\end{array}$ & $\begin{array}{l}\text { Constur } \\
\text { Type }\end{array}$ & $\begin{array}{l}\text { Struct. } \\
\text { Accidents }\end{array}$ & State & $\begin{array}{c}\text { NPH } \\
\text { Evaluation }\end{array}$ \\
\hline MO845 & LUNCHROOM & & 1 & & & 67 & & & Benton & \\
\hline $200 E$ & TRAILER@SLAB & $\mathrm{KEH}$ & HOLLADAY, J & FOUCAULT, DA & & & & & Washington & \\
\hline MO847 & RESTROOM & & 1 & & & 22 & & & Benton & \\
\hline $200 \mathrm{~W}$ & TRAILER ON & $\mathrm{KEH}$ & HOLLADAY, J & FOUCAULT, DA & & & & & Washington & \\
\hline MO848 & MOBILE OFFICE & & 1 & & & 22 & & & Benton & \\
\hline 3000 & $@$ SLAB YARD & $\mathrm{KEH}$ & WITHERSPOO & WITHERSPOON, & & & & & Washington & \\
\hline MO849 & CHANGE/LUNCHR & & 1 & & & 12 & & & Benton & \\
\hline $200 \mathrm{E}$ & OOM TRLR@ & $\mathrm{KEH}$ & WITHERSPOO & WITHERSPOON, & & & & & Washington & \\
\hline MO850 & MOBILE OFFICE & & 1 & & 21 & 268 & & & Benton & \\
\hline $200 E$ & E. OF $2704 \mathrm{HV}$ & TWR & KIMBROUGH, & ERMOLD, LEON & & & & & Washington & \\
\hline MO85I & MOBILE OFFICE & & 1 & & & 134 & & & Benton & \\
\hline 3000 & & TRP & & & & & & & Washington & \\
\hline $\mathrm{MO} 852$ & MOBILE OFFICE & & 1 & & 25 & 429 & & & Benton & \\
\hline $200 E$ & @ 4TH \& & $\mathrm{KEH}$ & TENVOOREN, & TENVOOREN, M & & & & & Washington & \\
\hline MO853 & MOBILE OFFICE & & 1 & & & 35 & & & Benton & \\
\hline $200 E$ & $@$ SLAB YARD & $\mathrm{KEH}$ & HOLLADAY, J & FOUCAULT, DA & & & & & Washington & \\
\hline MO854 & FIELD & & 1 & & & 51 & & & Benton & \\
\hline $100 \mathrm{~K}$ & CHANGEROOM & $\mathrm{KEH}$ & & WITHERSPOON, & & & & & Washington & \\
\hline MO858 & FIELD & & 1 & & & 106 & & & Benton & \\
\hline $200 E$ & CHANGE/LUNCHR & $\mathrm{KEH}$ & DEMPSEY, KEI & I DEMPSEY, KEIT & & & & & Washington & \\
\hline
\end{tabular}


WHC-SD-SQA-CSWD-501 Rev 0

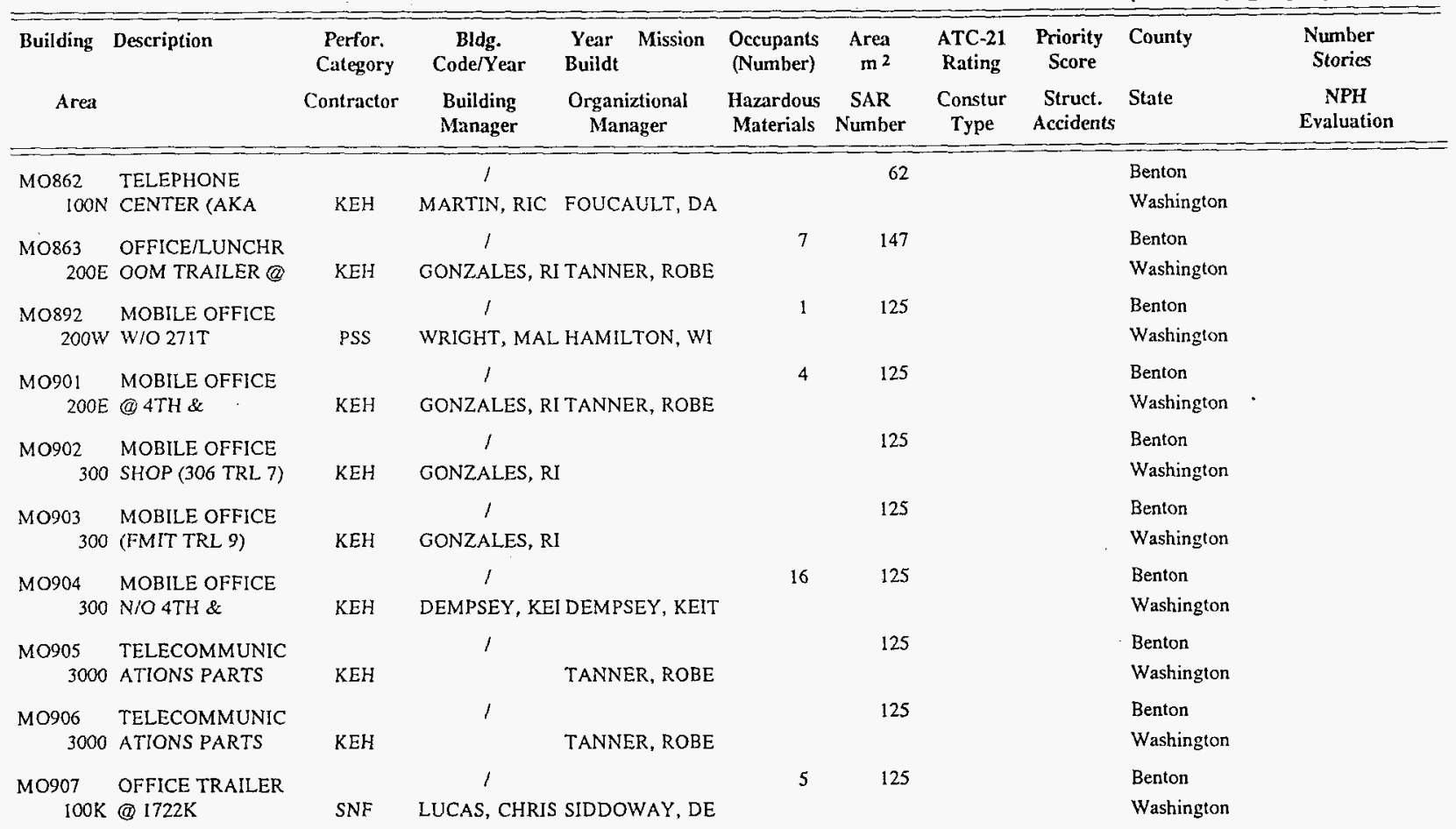


WHC-SD-SQA-CSWD-501 Rev 0

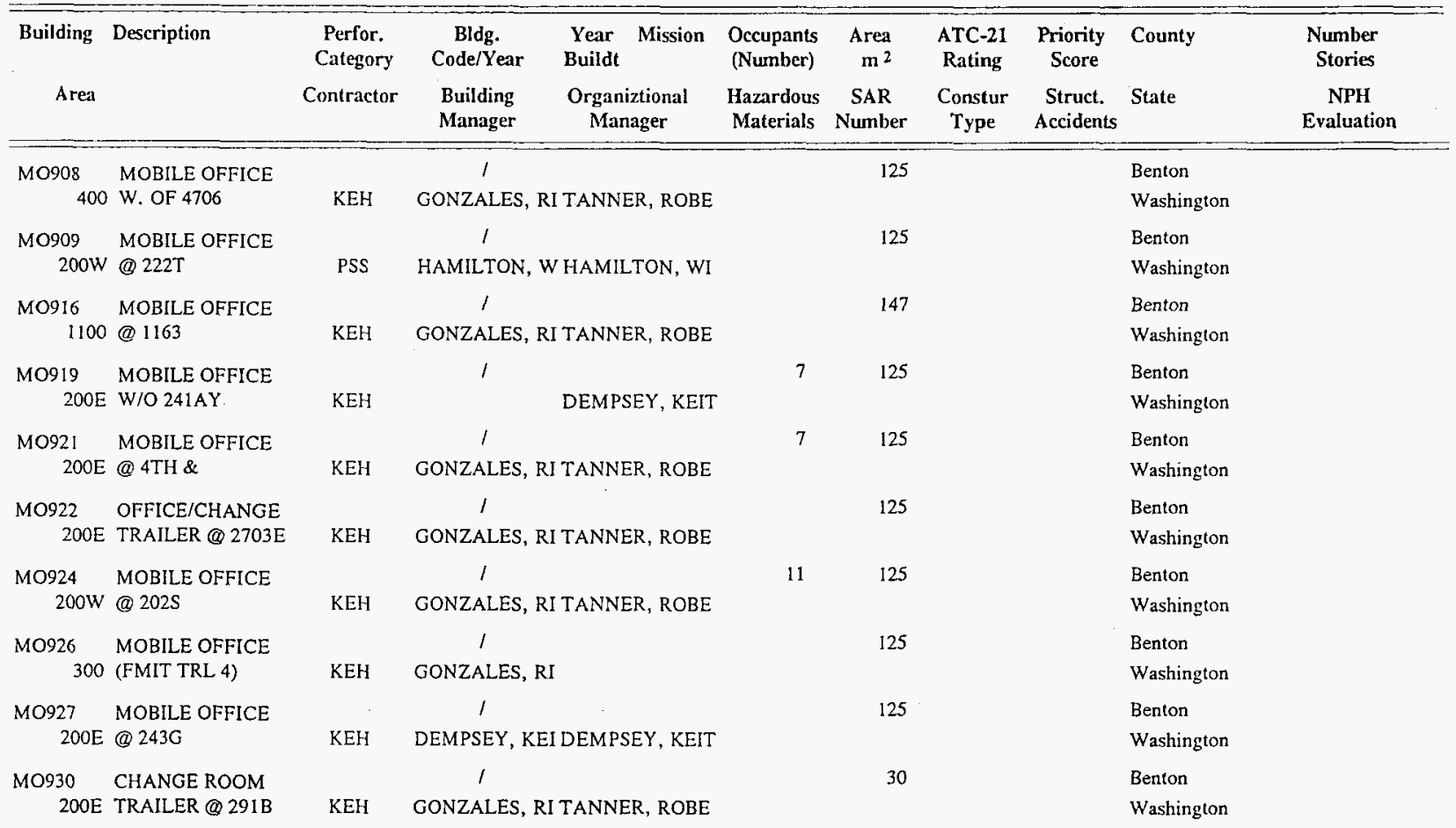


WHC.SD-SQA-CSWD-501 ReV 0

\begin{tabular}{|c|c|c|c|c|c|c|c|c|c|}
\hline Area & Description & $\begin{array}{l}\text { Perfor. } \\
\text { Category } \\
\text { Contractor }\end{array}$ & $\begin{array}{l}\text { Year Mission } \\
\text { Buildt } \\
\text { Organiztional } \\
\text { Manager }\end{array}$ & $\begin{array}{l}\text { Occupants } \\
\text { (Number) } \\
\text { Hazardous } \\
\text { Materials }\end{array}$ & $\begin{array}{c}\text { Area } \\
\text { m }^{2} \\
\text { SAR } \\
\text { Number }\end{array}$ & $\begin{array}{l}\text { ATC-21 } \\
\text { Rating } \\
\text { Constur } \\
\text { Type }\end{array}$ & $\begin{array}{l}\text { Priority } \\
\text { Score } \\
\text { Struct. } \\
\text { Accidents }\end{array}$ & State & $\begin{array}{c}\text { Number } \\
\text { Stories } \\
\text { NPH } \\
\text { Evaluation }\end{array}$ \\
\hline $\begin{array}{l}\text { MO931 } \\
200 E\end{array}$ & $\begin{array}{l}\text { GRAPHICS TRLR } \\
\text { @ 284E (ATT }\end{array}$ & $\mathrm{KEH}$ & $\begin{array}{c}\prime \\
\text { GONZALES, RI TANNER, ROBE }\end{array}$ & 4 & 134 & & & $\begin{array}{l}\text { Benton } \\
\text { Washington }\end{array}$ & \\
\hline $\begin{array}{l}\text { MOS32 } \\
200 E\end{array}$ & $\begin{array}{l}\text { MOBILE OFFICE } \\
@ 4 T H \&\end{array}$ & $\mathrm{KEH}$ & $\begin{array}{c}\prime \\
\text { GONZALES, RI TANNER, ROBE }\end{array}$ & 8 & 143 & & & $\begin{array}{l}\text { Benton } \\
\text { Washington }\end{array}$ & \\
\hline $\begin{array}{l}\text { MO933 } \\
300\end{array}$ & $\begin{array}{l}\text { MOBILE OFFICE } \\
\text { (FMIT TRL I0) }\end{array}$ & $\mathrm{KEH}$ & $\begin{array}{c}\prime \\
\text { GONZALES, RI }\end{array}$ & & 134 & & & $\begin{array}{l}\text { Benton } \\
\text { Washington }\end{array}$ & \\
\hline $\begin{array}{l}\text { MO934 } \\
200 \mathrm{E}\end{array}$ & $\begin{array}{l}\text { CHANGE/LUNCHR } \\
\text { OOM (PWRHSE }\end{array}$ & $\mathrm{KEH}$ & $\begin{array}{l}\text { / } \\
\text { GONZALES, RI TANNER, ROBE }\end{array}$ & 3 & 62 & & & $\begin{array}{l}\text { Benton } \\
\text { Washington }\end{array}$ & \\
\hline $\begin{array}{r}\text { MOS35 } \\
300\end{array}$ & $\begin{array}{l}\text { MOBILE OFFICE } \\
(3763 \text { TRL 1) }\end{array}$ & $\mathrm{KEH}$ & $\begin{array}{c}\text { / } \\
\text { GONZALES, RI }\end{array}$ & & 107 & & & $\begin{array}{l}\text { Benton } \\
\text { Washington }\end{array}$ & \\
\hline $\begin{array}{l}\text { MO936 } \\
200 \mathrm{~W}\end{array}$ & $\begin{array}{l}\text { MOBILE OFFICE } \\
\text { (a) } 222 \mathrm{~S}\end{array}$ & $\mathrm{KEH}$ & $\begin{array}{l}/ \\
\text { GONZALES, RI TANNER, ROBE }\end{array}$ & 7 & 147 & & & $\begin{array}{l}\text { Benton } \\
\text { Washington }\end{array}$ & \\
\hline $\begin{array}{l}\text { MO938 } \\
1100\end{array}$ & $\begin{array}{l}\text { MOBILE OFFICE } \\
\text { (a) } 1163\end{array}$ & $\mathrm{KEH}$ & $\begin{array}{l}/ \\
\text { GONZALES, RI TANNER, ROBE }\end{array}$ & & 147 & & & $\begin{array}{l}\text { Benton } \\
\text { Washington }\end{array}$ & \\
\hline $\begin{array}{l}\text { MO939 } \\
\quad 200 \mathrm{~W}\end{array}$ & $\begin{array}{l}\text { MOBILE OFFICE } \\
\text { (6) } 234-5 Z\end{array}$ & $\mathrm{KEH}$ & $\begin{array}{c}\text { I } \\
\text { GONZALES, RI TANNER, ROBE }\end{array}$ & 6 & 62 & & & $\begin{array}{l}\text { Benton } \\
\text { Washington }\end{array}$ & \\
\hline $\begin{array}{l}\text { MO940 } \\
\qquad 1100\end{array}$ & $\begin{array}{l}\text { MOBILE OFFICE } \\
\text { (d) } 1163\end{array}$ & $\mathrm{KEH}$ & $\begin{array}{c}\qquad \\
\text { GONZALES, RI TANNER, ROBE }\end{array}$ & 6 & 125 & & & $\begin{array}{l}\text { Benton } \\
\text { Washington }\end{array}$ & \\
\hline $\begin{array}{l}\text { MO94I } \\
\qquad 200 \mathrm{~W}\end{array}$ & $\begin{array}{l}\text { MOBILE FIELD } \\
\text { OFFICE @ 272WA }\end{array}$ & PSS & $\begin{array}{l}l \\
\text { PYZEL, DONA HAMILTON, WI }\end{array}$ & & 46 & & & $\begin{array}{l}\text { Benton } \\
\text { Washington }\end{array}$ & \\
\hline
\end{tabular}


WHC-SD-SQA-CSWD-501 Rev 0

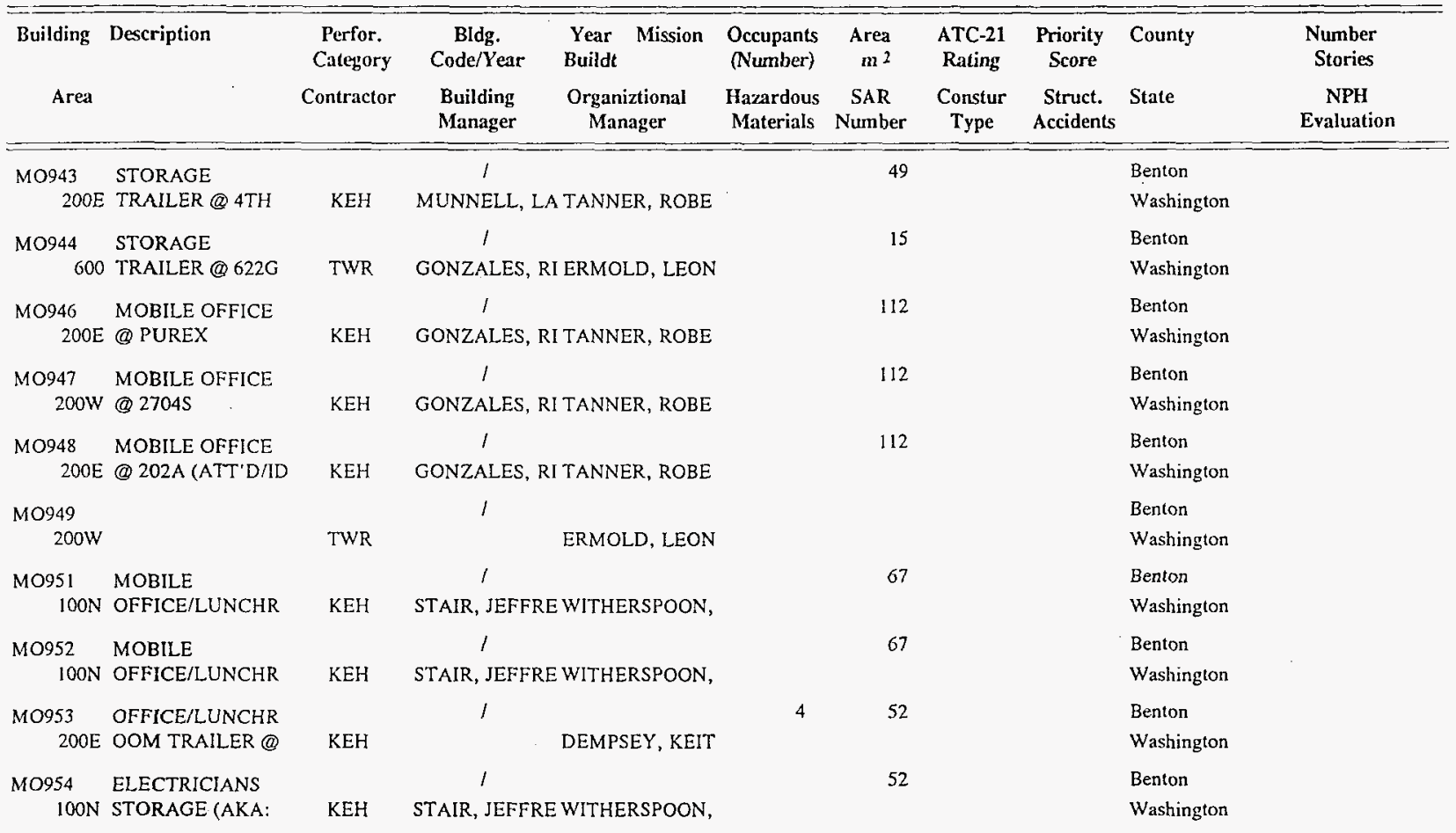


WHC-SD-SQA-CSWD-501 Rev 0

\begin{tabular}{|c|c|c|c|c|c|c|c|c|c|c|}
\hline Building & Description & $\begin{array}{l}\text { Perfor. } \\
\text { Category }\end{array}$ & $\begin{array}{c}\text { Bldg. } \\
\text { Code/Year }\end{array}$ & $\begin{array}{l}\text { Year Mission } \\
\text { Buildt }\end{array}$ & $\begin{array}{l}\text { Occupants } \\
\text { (Number) }\end{array}$ & $\begin{array}{r}\text { Area } \\
\mathrm{m}^{2}\end{array}$ & $\begin{array}{l}\text { ATC-2I } \\
\text { Rating }\end{array}$ & $\begin{array}{l}\text { Priority } \\
\text { Score }\end{array}$ & County & $\begin{array}{c}\text { Number } \\
\text { Stories }\end{array}$ \\
\hline Area & & Contractor & $\begin{array}{l}\text { Building } \\
\text { Manager }\end{array}$ & $\begin{array}{c}\text { Organiztional } \\
\text { Manager }\end{array}$ & $\begin{array}{l}\text { Hazardous } \\
\text { Materials }\end{array}$ & $\begin{array}{c}\text { SAR } \\
\text { Number }\end{array}$ & $\begin{array}{c}\text { Constur } \\
\text { Type }\end{array}$ & $\begin{array}{l}\text { Struct. } \\
\text { Accidents }\end{array}$ & State & $\begin{array}{c}\text { NPH } \\
\text { Evaluation }\end{array}$ \\
\hline M0955 & MOBILE OFFICE & & 1 & & & 112 & & & Benton & \\
\hline $200 \mathrm{E}$ & (G) UNSECURED & $\mathrm{KEH}$ & GONZALES, RI & I TANNER, ROBE & & & & & Washington & \\
\hline MO956 & LUNCHROOM/TR & & 1 & & & 134 & & & Benton & \\
\hline $200 \mathrm{~W}$ & AINING TRLR ON & $\mathrm{KEH}$ & HOLLADAY, J & FOUCAULT, DA & & & & & Washington & \\
\hline M0957 & MOBILE OFFICE & & 1 & & & 10 & & & Benton & \\
\hline $100 \mathrm{~N}$ & (CONSTRUCTION) & KEH & HOLLADAY, J & FOUCAULT, DA & & & & & Washington & \\
\hline MO958 & OFFICE/LUNCHR & & 1 & & & 60 & & & Benton & \\
\hline $200 E$ & OOM TRAILER@ & $\mathrm{KEH}$ & HOLLADAY, J & FOUCAULT, DA & & & & & Washington & \\
\hline MO959 & RESTROOM & & 1 & & & 22 & & & Benton & \\
\hline $200 \mathrm{E}$ & TRAILER @224B & $\mathrm{KEH}$ & HOLLADAY, J & FOUCAULT, DA & & & & . & Washington & \\
\hline MO961 & FIELD TRAILER & & 1 & & & 15 & & & Benton & \\
\hline $200 \mathrm{E}$ & @ 224B & $\mathrm{KEH}$ & FOUCAULT, D & FOUCAULT, DA & & & & & Washington & \\
\hline MO962 & CHANGE/LUNCHR & & 1 & & & 15 & & & Benton & \\
\hline $200 \mathrm{E}$ & OOM TRAILER @ & $\mathrm{KEH}$ & FOUCAULT, D & WITHERSPOON, & & & & & Washington & \\
\hline MO964 & MOBILE OFFICE & & 1 & & 2 & 67 & & & Benton & \\
\hline $200 \mathrm{E}$ & (1) 224B & $\mathrm{KEH}$ & HOLLADAY, J & FOUCAULT, DA & & & & & Washington & \\
\hline MO965 & STORAGE & & 1 & & & 52 & & & Benton & \\
\hline $200 \mathrm{E}$ & TRAILER@224B & KEH & HOLLADAY, J & FOUCAULT, DA & & & & & Washington & \\
\hline MO966 & LUNCHROOM & & 1 & & & 112 & & & Benton & \\
\hline $200 \mathrm{E}$ & TRAILER @ 224B & TWR & HOLLADAY, J & WICKS, JAMES & & & & & Washington & \\
\hline
\end{tabular}


WHC-SD-SQA-CSWD-501 Rev- 0

\begin{tabular}{|c|c|c|c|c|c|c|c|c|c|c|}
\hline Building & Description & $\begin{array}{c}\text { Perfor. } \\
\text { Category } \\
\text { Contractor }\end{array}$ & $\begin{array}{l}\text { Bldg. } \\
\text { Code/Year } \\
\text { Building } \\
\text { Manager }\end{array}$ & $\begin{array}{l}\text { Year Mission } \\
\text { Buildt } \\
\text { Organiztional } \\
\text { Manager }\end{array}$ & $\begin{array}{c}\text { Occupants } \\
\text { (Number) } \\
\text { Hazardous } \\
\text { Materials }\end{array}$ & $\begin{array}{c}\text { Area } \\
\mathrm{m}^{2} \\
\text { SAR } \\
\text { Number }\end{array}$ & $\begin{array}{c}\text { ATC-21 } \\
\text { Rating } \\
\text { Constur } \\
\text { Type }\end{array}$ & $\begin{array}{c}\text { Priority } \\
\text { Score } \\
\text { Struct. } \\
\text { Accidents }\end{array}$ & State & $\begin{array}{c}\text { Number } \\
\text { Stories } \\
\text { NPH } \\
\text { Evaluation }\end{array}$ \\
\hline $\begin{array}{l}\text { MO967 } \\
200 \mathrm{E}\end{array}$ & $\begin{array}{l}\text { MOBILE OFFICE } \\
\text { (1) } 224 \mathrm{~B}\end{array}$ & $\mathrm{KEH}$ & $\begin{array}{c}\prime \\
\text { HOLLADAY, J }\end{array}$ & FOUCAULT, DA & & 67 & & & $\begin{array}{l}\text { Benton } \\
\text { Washington }\end{array}$ & \\
\hline $\begin{array}{l}\text { MO968 } \\
200 E\end{array}$ & $\begin{array}{l}\text { CHANGE TRLR @ } \\
\text { 224B }\end{array}$ & $\mathrm{KEH}$ & $\begin{array}{c}/ \\
\text { FOUCAULT, D }\end{array}$ & FOUCAULT, DA & & 12 & & & $\begin{array}{l}\text { Benton } \\
\text { Washington }\end{array}$ & \\
\hline $\begin{array}{r}\text { MO969 } \\
100 \mathrm{~K}\end{array}$ & MOBILE OFFICE & SNF & $\begin{array}{c}\text { ' } \\
\text { MILLER, CHA }\end{array}$ & $\begin{array}{l}\text { 08/94 } \\
\text { SIDDOWAY, DE }\end{array}$ & & 312 & & & $\begin{array}{l}\text { Benton } \\
\text { Washington }\end{array}$ & \\
\hline $\begin{array}{l}\text { MO970 } \\
200 \mathrm{E}\end{array}$ & $\begin{array}{l}\text { MOBILE OFFICE } \\
\text { @ Q UNSECURED }\end{array}$ & $\mathrm{KEH}$ & $\begin{array}{c}/ \\
\text { GONZALES, RI }\end{array}$ & I TANNER, ROBE & 16 & 312 & & & $\begin{array}{l}\text { Benton } \\
\text { Washington }\end{array}$ & \\
\hline $\begin{array}{l}\text { MO971 } \\
200 E\end{array}$ & $\begin{array}{l}\text { MOBILE OFFICE } \\
@ \text { UNSECURED }\end{array}$ & $\mathrm{KEH}$ & $\begin{array}{c}/ \\
\text { GONZALES, RI }\end{array}$ & TANNER, ROBE & 13 & 312 & & & $\begin{array}{l}\text { Benton } \\
\text { Washington }\end{array}$ & \\
\hline $\begin{array}{r}\text { MO972 } \\
\quad 3000\end{array}$ & $\begin{array}{l}\text { MOBILE OFFICE } \\
\text { NEAR } 1226\end{array}$ & $\mathrm{KEH}$ & 1 & FOUCAULT, DA & & 15 & & & $\begin{array}{l}\text { Benton } \\
\text { Washington }\end{array}$ & \\
\hline $\begin{array}{l}\text { MO973 } \\
200 \mathrm{E}\end{array}$ & $\begin{array}{l}\text { STORAGE/CONST } \\
\text { RUCTION }\end{array}$ & $\mathrm{KEH}$ & $\frac{1}{\text { HUBBARD, BE }}$ & FOUCAULT, DA & & 51 . & & & $\begin{array}{l}\text { Benton } \\
\text { Washington }\end{array}$ & \\
\hline $\begin{array}{l}\text { MO974 } \\
200 \mathrm{E}\end{array}$ & $\begin{array}{l}\text { STORAGE } \\
\text { TRAILER } @ 241 \mathrm{~A}\end{array}$ & KEH & $\begin{array}{c}\prime \\
\text { DEMPSEY, KEI }\end{array}$ & DEMPSEY, KEIT & & 51 & & & $\begin{array}{l}\text { Benton } \\
\text { Washington }\end{array}$ & \\
\hline $\begin{array}{l}\text { MO975 } \\
200 \mathrm{E}\end{array}$ & $\begin{array}{l}\text { CHANGEROOM } \\
\text { TRAILER @ SLAB }\end{array}$ & KEH & $\begin{array}{c}\prime \\
\text { HOLLADAY, J }\end{array}$ & TANNER, ROBE & & 13 & & & $\begin{array}{l}\text { Benton } \\
\text { Washington }\end{array}$ & \\
\hline $\begin{array}{l}\text { MO976 } \\
200 E\end{array}$ & $\begin{array}{l}\text { CHANGEROOM } \\
\text { TRAILER @ } S\end{array}$ & KEH & $\begin{array}{c}I \\
\text { HOLLADAY, J }\end{array}$ & FOUCAULT, DA & & 13 & & & $\begin{array}{l}\text { Benton } \\
\text { Washington }\end{array}$ & \\
\hline
\end{tabular}


WHC-SD-SQA-CSWD-501 Rev 0

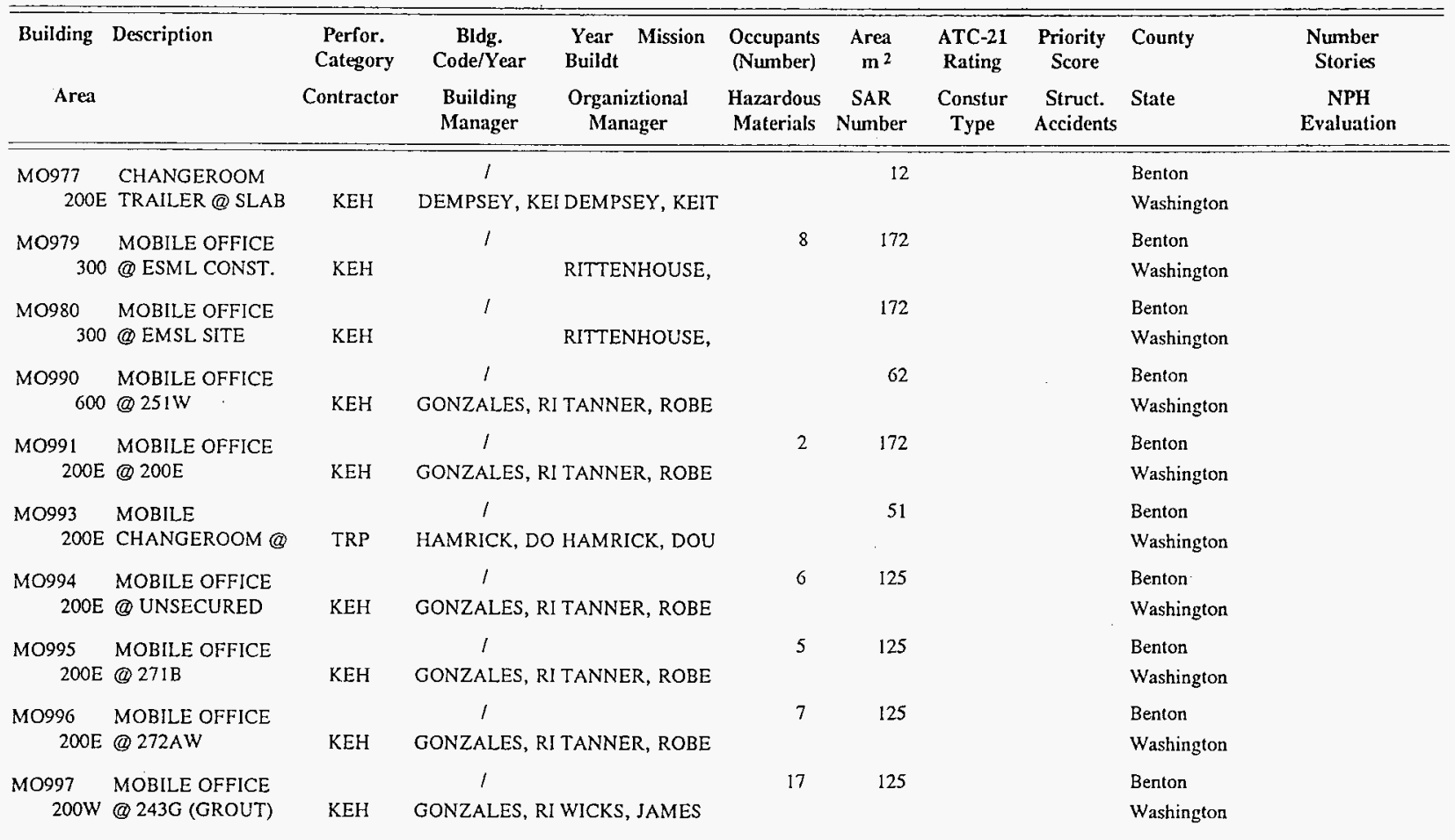


WHC-SD-SQA-CSWD-501 Rev 0

\begin{tabular}{|c|c|c|c|c|c|c|c|c|c|c|}
\hline Building & Description & $\begin{array}{l}\text { Perfor. } \\
\text { Category }\end{array}$ & $\begin{array}{l}\text { Bldg. } \\
\text { Code/Year }\end{array}$ & $\begin{array}{l}\text { Year Mission } \\
\text { Buildt }\end{array}$ & $\begin{array}{l}\text { Occupants } \\
\text { (Number) }\end{array}$ & $\begin{array}{r}\text { Area } \\
\mathrm{m}^{2}\end{array}$ & $\begin{array}{c}\text { ATC-21 } \\
\text { Rating }\end{array}$ & $\begin{array}{l}\text { Priority } \\
\text { Score }\end{array}$ & County & $\begin{array}{c}\text { Number } \\
\text { Stories }\end{array}$ \\
\hline Area & & Contractor & $\begin{array}{l}\text { Building } \\
\text { Manager }\end{array}$ & $\begin{array}{l}\text { Organiztional } \\
\text { Manager }\end{array}$ & $\begin{array}{l}\text { Hazardous } \\
\text { Materials }\end{array}$ & $\begin{array}{c}\text { SAR } \\
\text { Number }\end{array}$ & $\begin{array}{l}\text { Constur } \\
\text { Type }\end{array}$ & $\begin{array}{l}\text { Struct. } \\
\text { Accidents }\end{array}$ & State & $\begin{array}{c}\text { NPH } \\
\text { Evaluation }\end{array}$ \\
\hline $\begin{array}{r}\text { M0998 } \\
600\end{array}$ & $\begin{array}{l}\text { FIELD TRAILER } \\
\text { HTS YARD }\end{array}$ & PSS & $\begin{array}{c}\prime \\
\text { GARDNER, M }\end{array}$ & DILIBERTO, AN & & 24 & & & $\begin{array}{l}\text { Benton } \\
\text { Washington }\end{array}$ & \\
\hline
\end{tabular}


WHC-SD-SQA-CSWD-501 Rev 0

Appendix E

Facility Demolition List

\begin{tabular}{|c|c|c|}
\hline BLDG & $\begin{array}{l}\text { Demolition } \\
\text { Contract }\end{array}$ & SQFT \\
\hline 3765 & NO & 12160 \\
\hline $3726 \mathrm{~A}$ & NO & 144 \\
\hline $3710 \mathrm{~A}$ & NO & 270 \\
\hline $3734 A$ & NO & 780 \\
\hline 3734 & YES & 240 \\
\hline 3732 & YES & 1365 \\
\hline 3706 & YES & 7494 \\
\hline 3706A & UNK & 1510 \\
\hline 321 & YES & 27439 \\
\hline $2719 \mathrm{WA}$ & NO & 2269 \\
\hline $2720 \mathrm{~W}$ & NO & 2216 \\
\hline $2709 W$ & No & 1860 \\
\hline $274 W$ & NO & 3815 \\
\hline $2723 W$ & NO & 4515 \\
\hline $222 \mathrm{~T}$ & YES & 7531 \\
\hline $222 \mathrm{U}$ & YES & 7336 \\
\hline 2312 & YES & 38451 \\
\hline $2701 \mathrm{M}$ & NO & 278 \\
\hline $2704 C$ & NO & 1025 \\
\hline $2713 E$ & No & 4127 \\
\hline $2715 E$ & NO & 952 \\
\hline $2715 M$ & No & 952 \\
\hline $2719 E$ & NO & 833 \\
\hline 2722E & NO & 1200 \\
\hline 2701EC & NO & 132 \\
\hline $276 C$ & YES & 2300 \\
\hline
\end{tabular}

Appendix E-1 